\title{
Aquatic Insects from the Caatinga: checklists and diversity assessments of Ubajara (Ceará State) and Sete Cidades (Piauí State) National Parks, Northeastern Brazil
}

Daniela Maeda Takiya ${ }^{\ddagger}$, Allan Paulo Moreira Santos ${ }^{\S}$, Ângelo Parise Pintol, Ana Lucia HenriquesOliveira ${ }^{\ddagger}$, Alcimar do Lago Carvalhol, Brunno Henrique Lanzellotti Sampaio ${ }^{\ddagger}$, Bruno Clarkson, Felipe Ferraz Figueiredo Moreiraף, Fernanda Avelino-Capistrano ${ }^{\ddagger}$, Inês Corrêa Gonçalves ${ }^{\ddagger}$, Isabelle da Rocha Silva Cordeiro", Josenir Teixeira Câmara\#, Julianna Freires Barbosa ${ }^{\ddagger}$, W. Rafael Maciel de Souza ${ }^{\ddagger}$, José Albertino Rafael\#

\footnotetext{
‡ Laboratório de Entomologia, Departamento de Zoologia, Instituto de Biologia, Universidade Federal do Rio de Janeiro, Rio de Janeiro, Brazil

$\S$ Departamento de Zoologia, Universidade Federal do Estado do Rio de Janeiro, Rio de Janeiro, Brazil

| Laboratório de Biologia e Sistemática de Odonata (LABIOSIS), Departamento de Entomologia, Museu Nacional, Universidade Federal do Rio de Janeiro, Rio de Janeiro, Brazil

I Laboratório Nacional e Internacional de Referência em Taxonomia de Triatomíneos, Instituto Oswaldo Cruz, Fundação Oswaldo Cruz, Rio de Janeiro, Brazil

\# Coordenação de Biodiversidade, Instituto Nacional de Pesquisas da Amazônia, Manaus, Brazil
}

\section{Corresponding author: Daniela Maeda Takiya (takiya@gmail.com)}

Academic editor: Pavel Stoev

Received: 03 Mar 2016 | Accepted: 01 Jun 2016| Published: 05 Aug 2016

Citation: Takiya D, Santos A, Pinto Â, Henriques-Oliveira A, Carvalho A, Sampaio B, Clarkson B, Moreira F, Avelino-Capistrano F, Gonçalves I, Cordeiro I, Câmara J, Barbosa J, de Souza W, Rafael J (2016) Aquatic Insects from the Caatinga: checklists and diversity assessments of Ubajara (Ceará State) and Sete Cidades (Piauí State) National Parks, Northeastern Brazil. Biodiversity Data Journal 4: e8354. doi: 10.3897/BDJ.4.e8354

\begin{abstract}
Background

Diversity and distribution of Neotropical aquatic insects is still poorly known, with many species to be recorded and many others to be described, due to the small number of taxonomists and sparse faunistic studies. This knowledge is especially poor in the


Caatinga Domain in Northeastern Brazil, even though, this region may have played an important historical role in the spatial evolution of faunas of forested areas in northern South America.

\section{New information}

Aquatic insect checklists of 96 species from Parque Nacional de Ubajara (Ceará State, Brazil) and 112 species from Parque Nacional de Sete Cidades (Piauí State, Brazil) are presented, representing the following taxa: Elmidae, Epimetopidae, Hydrophilidae, and Torridincolidae (Coleoptera), Hemerodromiinae (Diptera: Empididae), Ephemeroptera, Gerromorpha and Nepomorpha (Hemiptera), Odonata, Plecoptera, and Trichoptera. Because of the scarce number of biological inventories in Northeastern Brazil, several new distributional records (of species, genera, and families) for Brazil, Northeastern Brazil, and Ceará and Piauí states are provided. In addition, several undescribed species were detected, being 26 from Ubajara and 20 from Sete Cidades. Results represent a significant increase to the known fauna of these states, ranging from $13 \%-70 \%$ increase for Ceará and $41 \%$ to $91 \%$ increase for Piauí. Although both parks are relatively close to each other and within the Caatinga domain, their aquatic fauna display a very high complementarity (89\% species), possibly due to structural differences of water bodies sampled in each park. Rarefaction curves based on quantitative light trap samples suggest a much higher expected species richness of aquatic insects at Sete Cidades than at Ubajara National Park. Discussion on biogeographical affinities of this sample of the Caatinga fauna is provided.

\section{Keywords}

Species richness, Amazonia, Cerrado, Atlantic forest, Freshwater macroinvertebrates

\section{Introduction}

\section{Aquatic insects}

Insects constitute the most diverse animal group and represent one of the earliest lineages occupying the terrestrial habitat (Grimaldi and Engel 2005). Although they are mostly terrestrial, insects usually dominate inland waterbodies in terms of species number and biomass. Many terrestrial insects are resilient to eventual submersion in water (Chapman 1998), but true aquatic insects inhabit freshwater or marine environments at least for one life stage and have several morphological, physiological, and behavioural adaptations. Aquatic insects are usually abundant and important components of energy flow in freshwater habitats, and thus have been the focus of many ecological studies, as well as, the most frequent macroinvertebrate group used in biomonitoring (Rosenberg and Resh 1993, Merritt and Cummins 1996). 
The diversity of aquatic insects is a result of several independent invasions of aquatic habitats by terrestrial lineages (more than 50 separate invasions, according to Dijkstra et al. (2014). Marine insects are relatively rare, most of them living in coastal areas and only very few species in the open sea (Cheng 1976). On the other hand, insects inhabiting freshwater are close to 100,000 described species, in twelve insect orders, five of them primarily aquatic: Ephemeroptera, Odonata, Plecoptera, Megaloptera, and Trichoptera (Dijkstra et al. 2014).

Regional knowledge of the aquatic insect fauna is essential to ecological surveys or biomonitoring. In the Neotropics, diversity and distribution of aquatic insects is still poorly known, with many species to be recorded and many others to be described, especially because of the small number of taxonomists and sparse faunistic studies, even near large urban areas. Currently, there are approximately 7,000 described species of aquatic insects in Brazil (Boeger et al. 2016), representing approximately $8 \%$ of known species in the world. Amongst these groups, only Odonata and Psychodomorpha reach numbers higher than $14 \%$ of the world's fauna (Table 1). The number of species of some aquatic taxa recorded from Brazil, e.g., Trichoptera, is far below the average of $8 \%$ of the world fauna, very likely due to the many undescribed species waiting to be formally named or recorded from the country. Undersampling is one of the major biases for richness evaluations studies and conservation actions such as recently demonstrated for Brazilian dragonflies (Vianna and De Marco Júnior 2012). Therefore, local inventories should remain a priority, particularly in those poorly sampled areas, such as Northeastern Brazil.

Table 1.

Number of described species in the major aquatic insect groups in the world and in Brazil, and percentage of Brazilian fauna in relation to the world's biota.

\begin{tabular}{|c|c|c|c|}
\hline Taxon & World $^{a}$ & Brazil $^{\mathbf{b}}$ & Brazilian fauna / World fauna \\
\hline Ephemeroptera & 3,046 & 339 & $11.1 \%$ \\
\hline Odonata & 5,952 & 856 & $14.4 \%$ \\
\hline Hemiptera: Nepomorpha & 2,404 & 301 & $12.5 \%$ \\
\hline Hemiptera: Gerromorpha* & 2,021 & 228 & $11.3 \%$ \\
\hline Plecoptera & 3,497 & 164 & $4.7 \%$ \\
\hline Diptera: Culicomorpha & 19,618 & 1,719 & $8.8 \%$ \\
\hline Diptera: Ephydridae & 1,994 & 139 & $7.0 \%$ \\
\hline Diptera: Psychodomorpha & 3,412 & 519 & $15.2 \%$ \\
\hline Diptera: Tipulomorpha & 15,770 & 648 & $4.1 \%$ \\
\hline Diptera: Tabanomorpha & 5,373 & 498 & $9.3 \%$ \\
\hline Trichoptera & 14,291 & 687 & $4.8 \%$ \\
\hline Megaloptera & 328 & 20 & $6.1 \%$ \\
\hline
\end{tabular}




\begin{tabular}{|l|l|l|l|}
\hline Coleoptera: Elmidae & 1,300 & 148 & $11.4 \%$ \\
\hline Coleoptera: Hydradephaga & 5,126 & 529 & $10.3 \%$ \\
\hline Coleoptera: Hydraenidae & 1,380 & 27 & $2.0 \%$ \\
\hline Coleoptera: Hydrophiloidea & 2,205 & 280 & $12.7 \%$ \\
\hline Coleoptera: Scirtidae & 1,330 & 45 & $3.4 \%$ \\
\hline Total & $\mathbf{8 9 , 0 4 7}$ & $\mathbf{7 , 1 4 7}$ & $\mathbf{8 . 0 \%}$ \\
\hline
\end{tabular}

${ }^{a}$ Numbers obtained from Dijkstra et al. (2014) (except Elmidae, from Jäch and Balke 2008). bNumbers obtained from Boeger et al. (2016).

*Gerromorpha are semiaquatic.

\section{The Caatinga Domain in Northeastern Brazil}

The Caatinga Domain (Coutinho 2006) extends for an area of approximately $800,000 \mathrm{~km} 2$ in Northeastern Brazil (Fig. 1), including parts of the Brazilian states Piauí (PI), Ceará (CE), Rio Grande do Norte (RN), Paraíba (PB), Pernambuco (PE), Alagoas (AL), Sergipe (SE), Bahia (BA), and Minas Gerais (MG). It is a mosaic of different forest types, characterized by short trees and shrubs with xerophytic characteristics, influenced by climatic extremes when compared to other Brazilian formations: highest solar radiation, highest annual temperature mean, lowest rates of relative humidity, and lowest precipitation, which is limited to a very short period of the year (Prado 2003). It is estimated that over $28 \%$ of the original Caatinga vegetation has been altered by human activities, such as, slash-and-burn agriculture, harvesting of firewood, hunting, and herding (Castelletti et al. 2003). Currently, only less than $2 \%$ of its natural vegetation is enclosed under federal protected areas.

Low diversity and low numbers of endemic species is traditionally assigned to the Caatinga, although recent studies suggest otherwise, highlighting it as an important component of Brazilian biodiversity (Leal et al. 2005). The Caatinga lies in between two of the world's biodiversity hotspots (Myers et al. 2000, Mittermeier et al. 2011), the Atlantic Forest and the Cerrado, and shares many biotic components with these domains. Current assessments of the Caatinga's biodiversity is certainly underestimated because few biological inventories have focused this region, e.g., for woody plants, $41 \%$ of its area has never been surveyed by scientists and $80 \%$ of what has been was surveyed poorly (Tabarelli and Vicente 2004). This scenario is certainly worse when it comes to general insect inventories.

\section{Previous records of aquatic insects in Northeastern Brazil}

Due to the lack of collecting efforts in the Caatinga and Northeastern Brazil, and consequent scarcity of available voucher material from Ceará and Piauí states in insect collections, any information on insect diversity from inventories in these regions will probably constitute new records from these Brazilian states. An up to date list of Coleoptera (Hydrophilidae), Ephemeroptera, Hemiptera (Gerromorpha and Nepomorpha), 
Odonata, and Trichoptera recorded from Brazilian states of Ceará (CE) and Piauí (PI) are given in Table 2. This table includes species records previously published based on material collected during this project. Previous to this study, there were no records of Elmidae, Epimetopidae, and Torridincolidae (Coleoptera), Leptophlebiidae (Ephemeroptera), Gelastocoridae, Naucoridae, and Pleidae (Hemiptera), and Ecnomidae, Polycentropodidae, and Xyphocentronidae (Trichoptera) for these Brazilian states. Part of distributional data were initially compiled based on available information at Moreira et al. (2011), Moreira (2016), Salles et al. (2016), and Santos et al. (2016). Curiously, the first record of an aquatic insect from either state was of Nephepeltia phryne from Piauí described by Perty (1834) (Fig. 2) and, amazingly, a second dragonfly species was only recorded to this state after 160 years in 1994.

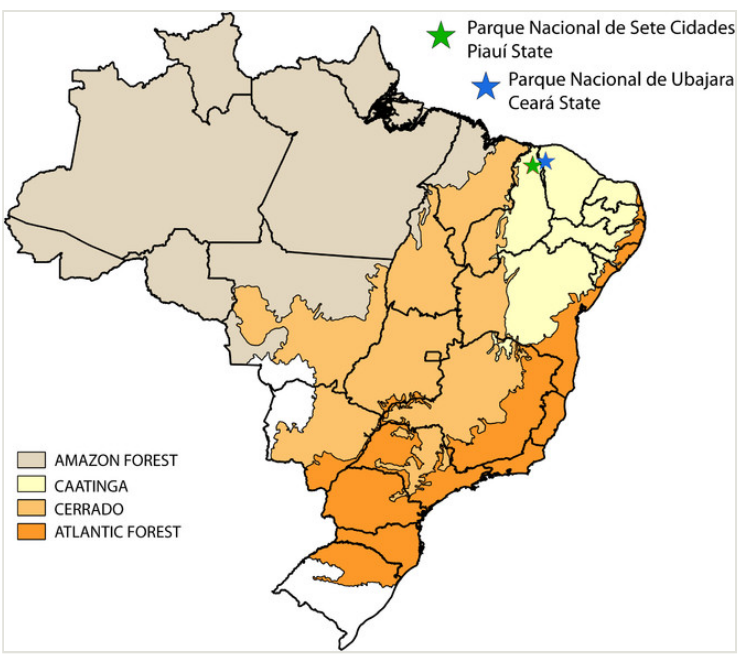

Figure 1.

Map of Brazilian states colored by four major phytogeographical domains: Amazon forest, Cerrado, Atlantic forest, and Caatinga; the last one including Ubajara (blue star) and Sete Cidades (green star) National Parks.

Table 2.

Species list of Coleoptera (Hydrophilidae), Ephemeroptera, Hemiptera (Gerromorpha and Nepomorpha), Odonata, and Trichoptera recorded from Ceará (CE) and Piauí (PI) states. Species marked with a "\#" were described or firstly recorded to these states based on material sampled in this project.

\begin{tabular}{|l|l|l|l|}
\hline Insect family & Species & $\begin{array}{l}\text { Previous } \\
\text { record }\end{array}$ & Reference \\
\hline COLEOPTERA & & & \\
\hline Hydrophilidae & Berosus auriceps Boheman, 1858 & CE & Orchymont 1943 \\
\hline & Berosus festivus Berg, 1885 & CE & Orchymont 1943 \\
\hline
\end{tabular}




\begin{tabular}{|c|c|c|}
\hline Berosus geayi Orchymont, 1937 & CE, PI & Orchymont 1943 \\
\hline Berosus novatus Orchymont, 1940 & $\mathrm{PI}$ & Orchymont 1943 \\
\hline Berosus patruelis Berg, 1885 & $\mathrm{PI}$ & Orchymont 1943 \\
\hline $\begin{array}{l}\text { Berosus truncatipennis } \\
\text { Castelnau, } 1840\end{array}$ & CE, PI & Orchymont 1943 \\
\hline Crenitulus solstitialis (Kirsch, 1873) & CE & Orchymont 1943 \\
\hline Crenitulus suturalis (LeConte, 1866) & CE & Komarek 2005 \\
\hline Dactylosternum punctigerum Knisch, 1924 & CE & Knisch 1924 \\
\hline Derallus altus (LeConte, 1855) & CE, PI & Orchymont 1943 \\
\hline Derallus ambitus Orchymont, 1940 & CE & Orchymont 1943 \\
\hline Derallus angustatus Sharp, 1882 & CE & Orchymont 1943 \\
\hline Derallus anicatus Orchymont, 1940 & $\mathrm{PI}$ & Orchymont 1943 \\
\hline $\begin{array}{l}\text { Enochrus (Methydrus) atlantis Orchymont, } \\
1943\end{array}$ & $\mathrm{PI}$ & Orchymont 1943 \\
\hline Hemiosus mornarius Orchymont, 1940 & CE & Orchymont 1943 \\
\hline Hemiosus mulvianus Orchymont, 1940 & CE, PI & Orchymont 1943 \\
\hline Hydrobiomorpha tricornis Mouchamps, 1959 & CE & Mouchamps 1959 \\
\hline Paracymus rufocinctus Bruch, 1915 & CE, PI & Orchymont 1943 \\
\hline $\begin{array}{l}\text { Phaenonotum convexoides Orchymont, } \\
1943\end{array}$ & CE & Orchymont 1943 \\
\hline $\begin{array}{l}\text { Tropisternus (Pleurhomus) sahlbergi (Sharp, } \\
\text { 1883) }\end{array}$ & CE, PI & Orchymont 1943 \\
\hline $\begin{array}{l}\text { Tropisternus (Pristoternus) apicipalpis } \\
\text { (Chevrolat,1834) }\end{array}$ & CE, PI & Orchymont 1943 \\
\hline $\begin{array}{l}\text { Tropisternus (Pristoternus) laevis (Sturm, } \\
1826 \text { ) }\end{array}$ & CE, PI & Orchymont 1943 \\
\hline $\begin{array}{l}\text { Tropisternus (Pristoternus) mutatus } \\
\text { Orchymont, } 1921\end{array}$ & CE & Orchymont 1943 \\
\hline $\begin{array}{l}\text { Tropisternus (Pristoternus) ovalis } \\
\text { (Castelnau, 1840) }\end{array}$ & CE, PI & Orchymont 1943 \\
\hline $\begin{array}{l}\text { Tropisternus (Pristoternus) regimbarti } \\
\text { Orchymont, } 1921\end{array}$ & CE, PI & Orchymont 1943 \\
\hline $\begin{array}{l}\text { Tropisternus (Strepitornus) collaris } \\
\text { (Fabricius, 1775) }\end{array}$ & CE, PI & Orchymont 1943 \\
\hline
\end{tabular}




\begin{tabular}{|c|c|c|c|}
\hline DIPTERA & & & \\
\hline \multirow[t]{4}{*}{ Empididae } & $\begin{array}{l}\text { \#Hemerodromia brevicercata Câmara, } \\
\text { Takiya, Plant \& Rafael, } 2015\end{array}$ & CE & Câmara et al. 2015 \\
\hline & $\begin{array}{l}\text { \#Hemerodromia membranosa Câmara, } \\
\text { Takiya, Plant \& Rafael, } 2015\end{array}$ & CE & Câmara et al. 2015 \\
\hline & $\begin{array}{l}\text { \#Hemerodromia mourai Câmara, Takiya, } \\
\text { Plant \& Rafael, } 2015\end{array}$ & CE & Câmara et al. 2015 \\
\hline & $\begin{array}{l}\text { \#Hemerodromia ubajaraensis Câmara, } \\
\text { Takiya, Plant \& Rafael, } 2015\end{array}$ & CE & Câmara et al. 2015 \\
\hline \multicolumn{4}{|c|}{ EPHEMEROPTERA } \\
\hline \multirow[t]{9}{*}{ Baetidae } & $\begin{array}{l}\text { Americabaetis alphus Lugo-Ortiz \& } \\
\text { McCafferty, } 1996\end{array}$ & CE, PI & Boldrini et al. 2012 \\
\hline & $\begin{array}{l}\text { Callibaetis pollens Needham and Murphy, } \\
1924\end{array}$ & CE, PI & Boldrini et al. 2012 \\
\hline & Callibaetis guttatus Navás, 1915 & CE & Cruz et al. 2014 \\
\hline & $\begin{array}{l}\text { Camelobaetidius cayumba (Traver \& } \\
\text { Edmunds, 1968) }\end{array}$ & CE, PI & Boldrini et al. 2012 \\
\hline & $\begin{array}{l}\text { Camelobaetidius janae Dominique \& } \\
\text { Thomas, } 2000\end{array}$ & $\mathrm{PI}$ & Boldrini et al. 2012 \\
\hline & $\begin{array}{l}\text { Camelobaetidius tuberosus Lugo-Ortiz \& } \\
\text { McCafferty, } 1999\end{array}$ & CE, PI & Boldrini et al. 2012 \\
\hline & Cloeodes irvingi Waltz \& McCafferty, 1987 & CE & Boldrini et al. 2012 \\
\hline & $\begin{array}{l}\text { Paracloeodes pacawara Nieto \& Salles, } \\
2006\end{array}$ & $\mathrm{PI}$ & Boldrini et al. 2012 \\
\hline & Paracloeodes waimiri Nieto \& Salles, 2006 & CE, PI & Boldrini et al. 2012 \\
\hline \multirow[t]{2}{*}{ Leptohyphidae } & $\begin{array}{l}\text { Traverhyphes (Mocoihyphes) yuati Molineri, } \\
2004\end{array}$ & $\mathrm{PI}$ & Cruz et al. 2011 \\
\hline & Tricorythodes mirca Molineri, 2002 & $\mathrm{PI}$ & Cruz et al. 2011 \\
\hline Polymitarcyidae & $\begin{array}{l}\text { Campsurus violaceus Needham \& Murphy, } \\
1924\end{array}$ & PI & Molineri et al. 2015 \\
\hline \multicolumn{4}{|l|}{ HEMIPTERA } \\
\hline \multirow[t]{3}{*}{ Gerridae } & $\begin{array}{l}\text { Brachymetra albinervis albinervis (Amyot \& } \\
\text { Serville, 1843) }\end{array}$ & CE & Dias da Rocha 1908 \\
\hline & Brachymetra furva Drake, 1957 & $\mathrm{PI}$ & Rodrigues et al. 2012 \\
\hline & Halobatopsis platensis (Berg, 1879) & $\mathrm{PI}$ & Rodrigues et al. 2012 \\
\hline
\end{tabular}




\begin{tabular}{|c|c|c|c|}
\hline & $\begin{array}{l}\text { \#Limnogonus profugus Drake \& Harris, } \\
1930\end{array}$ & CE & $\begin{array}{l}\text { Cordeiro and Moreira } \\
2015\end{array}$ \\
\hline & \#Neogerris lubricus (White, 1879) & $\mathrm{PI}$ & $\begin{array}{l}\text { Cordeiro and Moreira } \\
2015\end{array}$ \\
\hline & $\begin{array}{l}\text { Rheumatobates crassifemur schroederi } \\
\text { Hungerford, } 1954\end{array}$ & CE & Hungerford 1954 \\
\hline & Tachygerris adamsoni (Drake, 1942) & $\mathrm{PI}$ & Rodrigues et al. 2012 \\
\hline \multirow[t]{2}{*}{ Mesoveliidae } & Mesovelia amoena Uhler, 1894 & CE & $\begin{array}{l}\text { Moreira and Campos } \\
2012\end{array}$ \\
\hline & Mesovelia mulsanti White, 1879 & CE & $\begin{array}{l}\text { Moreira and Campos } \\
2012\end{array}$ \\
\hline \multirow[t]{5}{*}{ Veliidae } & $\begin{array}{l}\text { \#Microvelia ayacuchana Drake \& Maldonado } \\
\text { Capriles, } 1952\end{array}$ & PI & $\begin{array}{l}\text { Cordeiro and Moreira } \\
2015\end{array}$ \\
\hline & Microvelia mimula White, 1879 & CE & $\begin{array}{l}\text { Moreira and Campos } \\
2012\end{array}$ \\
\hline & \#Microvelia pulchella Westwood, 1834 & PI & $\begin{array}{l}\text { Cordeiro and Moreira } \\
2015\end{array}$ \\
\hline & \#Platyvelia brachialis (Stål, 1860) & PI & $\begin{array}{l}\text { Cordeiro and Moreira } \\
2015\end{array}$ \\
\hline & \#Rhagovelia whitei (Breddin, 1898) & CE & $\begin{array}{l}\text { Cordeiro and Moreira } \\
2015\end{array}$ \\
\hline \multirow[t]{7}{*}{ Belostomatidae } & Belostoma anurum (Herrich-Schäffer, 1848) & CE & Lauck 1962 \\
\hline & Belostoma dallasi De Carlo, 1930 & CE & Ribeiro 2007 \\
\hline & Belostoma dentatum (Mayr, 1863) & $\mathrm{PI}$ & Ribeiro 2007 \\
\hline & Belostoma elongatum Montandon, 1908 & $\mathrm{PI}$ & Ribeiro 2007 \\
\hline & Belostoma foveolatum (Mayr, 1863) & CE & Ribeiro 2000 \\
\hline & Belostoma micantulum (Stål, 1860) & CE & Dias da Rocha 1908 \\
\hline & Lethocerus maximus De Carlo, 1938 & CE & Dias da Rocha 1936 \\
\hline \multirow[t]{2}{*}{ Corixidae } & $\begin{array}{l}\text { Heterocorixa wrighti wrigthi Hungerford, } \\
1948\end{array}$ & CE & Hungerford 1948 \\
\hline & Tenagobia incerta Lundblad, 1929 & CE & Nieser 1977 \\
\hline Nepidae & Curicta montei De Carlo, 1960 & CE & Keffer 1997 \\
\hline \multirow[t]{3}{*}{ Notonectidae } & Buenoa amnigenus (White, 1879) & CE & Truxal 1953 \\
\hline & Buenoa salutis Kirkaldy, 1904 & CE & Truxal 1953 \\
\hline & Buenoa tarsalis Truxal, 1953 & CE & Truxal 1953 \\
\hline
\end{tabular}




\begin{tabular}{|c|c|c|c|}
\hline & Buenoa unguis Truxal, 1953 & CE & Truxal 1953 \\
\hline & Martarega brasiliensis Truxal, 1949 & CE & Truxal 1949 \\
\hline & Martarega bentoi Truxal, 1949 & $\mathrm{PI}$ & $\begin{array}{l}\text { Barbosa and Rodrigues } \\
2013\end{array}$ \\
\hline & \#Notonecta disturbata Hungerford, 1926 & $\mathrm{PI}$ & $\begin{array}{l}\text { Barbosa and } \\
\text { Nessimian } 2013\end{array}$ \\
\hline Ochteridae & \#Ochterus santosi Cordeiro \& Moreira, 2014 & $\mathrm{PI}$ & Cordeiro et al. 2014 \\
\hline \multicolumn{4}{|l|}{ ODONATA } \\
\hline \multirow[t]{4}{*}{ Aeshnidae } & Anax amazili (Burmeister, 1839) & CE & Navás 1924 \\
\hline & Castoraeschna januaria (Hagen, 1867) & CE & Navás 1924 \\
\hline & Coryphaeschna viriditas Calvert, 1952 & CE & Navás 1924 \\
\hline & Gynacantha nervosa Rambur, 1842 & CE & Navás 1924 \\
\hline \multirow[t]{4}{*}{ Gomphidae } & Cacoides latro (Erichson, 1848) & CE & Pinto 2010 \\
\hline & $\begin{array}{l}\text { Phyllocycla cf. gladiata (Hagen in Selys, } \\
1854 \text { ) }\end{array}$ & CE & $\begin{array}{l}\text { Nobre and Carvalho } \\
2014\end{array}$ \\
\hline & Progomphus dorsopallidus Byers, 1934 & CE & $\begin{array}{l}\text { Nobre and Carvalho } \\
2014\end{array}$ \\
\hline & \#Progomphus complicatus Selys, 1854 & CE & De Almeida et al. 2013 \\
\hline \multirow[t]{12}{*}{ Libellulidae } & Brachymesia furcata (Hagen, 1861) & CE & Navás 1924 \\
\hline & Brachymesia herbida (Gundlach, 1889) & CE & Pinto 2010 \\
\hline & $\begin{array}{l}\text { Brechmorhoga praecox praecox (Hagen, } \\
\text { 1861) }\end{array}$ & CE & $\begin{array}{l}\text { Nobre and Carvalho } \\
2014\end{array}$ \\
\hline & Dasythemis esmeralda Ris, 1910 & CE & $\begin{array}{l}\text { Nobre and Carvalho } \\
2014\end{array}$ \\
\hline & Diastatops obscura (Fabricius, 1775) & CE & Navás 1924 \\
\hline & Dythemis cf. nigra Martin, 1897 & CE & $\begin{array}{l}\text { Nobre and Carvalho } \\
2014\end{array}$ \\
\hline & Dythemis velox Hagen, 1861 & CE & Navás 1924 \\
\hline & Erythemis peruviana (Rambur, 1842) & CE & Pinto 2010 \\
\hline & Erythemis plebeja (Burmeister, 1839) & CE & Navás 1924 \\
\hline & Erythemis vesiculosa (Fabricius, 1775) & CE & Pinto 2010 \\
\hline & Erythrodiplax basalis (Kirby, 1897) & CE & Navás 1924 \\
\hline & Erythrodiplax fusca (Rambur, 1842) & CE & Navás 1924 \\
\hline
\end{tabular}




\begin{tabular}{|c|c|c|}
\hline Erythrodiplax latimaculata Ris, 1911 & CE & $\begin{array}{l}\text { Nobre and Carvalho } \\
2014\end{array}$ \\
\hline Erythrodiplax leticia Machado, 1996 & CE, PI & $\begin{array}{l}\text { Nobre and Carvalho } \\
2014 \text {, Nobre } 2016\end{array}$ \\
\hline Erythrodiplax ochracea (Burmeister, 1839) & CE & Navás 1924 \\
\hline Erythrodiplax paraguayensis (Förster, 1905) & CE & Pinto 2010 \\
\hline Erythrodiplax sp. & CE & $\begin{array}{l}\text { Nobre and Carvalho } \\
2014\end{array}$ \\
\hline Erythrodiplax umbrata (Linnaeus, 1758) & $\mathrm{CE}$ & Navás 1924 \\
\hline Idiataphe amazonica (Kirby, 1889) & CE & Pinto 2010 \\
\hline Idiataphe cubensis (Scudder, 1866) & CE & Navás 1924 \\
\hline Macrothemis griseofrons (Calvert, 1909) & CE & Navás 1916 \\
\hline Macrothemis hemichlora Burmeister, 1839 & CE & Navás 1924 \\
\hline Macrothemis lutea Calvert, 1909 & CE & $\begin{array}{l}\text { Nobre and Carvalho } \\
2014\end{array}$ \\
\hline Miathyria marcella (Selys in Sagra, 1857) & CE & Navás 1924 \\
\hline Micrathyria debilis (Hagen, 1861) & CE & Navás 1924 \\
\hline Micrathyria hesperis (Ris, 1911) & CE, PI & $\begin{array}{l}\text { Navás } 1924 \text {, Assis and } \\
\text { Costa } 1994\end{array}$ \\
\hline Micrathyria ocellata Martin, 1897 & CE & Navás 1924 \\
\hline Micrathyria tibialis Kirby, 1897 & CE & Navás 1924 \\
\hline Nephepeltia phryne (Perty, 1834) & $\mathrm{PI}$ & Perty 1834 \\
\hline Oligoclada sylvia (Kirby, 1899) & CE & Kirby 1889 \\
\hline Orthemis aequilibris (Calvert, 1909) & CE & $\begin{array}{l}\text { Nobre and Carvalho } \\
2014\end{array}$ \\
\hline Orthemis aff. sulphurata Hagen, 1868 & CE & Pinto 2010 \\
\hline Orthemis ferruginea-group & CE & $\begin{array}{l}\text { Nobre and Carvalho } \\
2014\end{array}$ \\
\hline Orthemis flavopicta Kirby, 1889 & CE & $\begin{array}{l}\text { Nobre and Carvalho } \\
2014\end{array}$ \\
\hline Pantala flavescens (Fabricius, 1798) & CE & $\begin{array}{l}\text { Nobre and Carvalho } \\
2014\end{array}$ \\
\hline Perithemis bella Kirby, 1889 & $\mathrm{CE}$ & Navás 1924 \\
\hline
\end{tabular}




\begin{tabular}{|c|c|c|c|}
\hline & Perithemis mooma Kirby, 1889 & CE, PI & $\begin{array}{l}\text { Navás 1924, Costa et } \\
\text { al. } 2006\end{array}$ \\
\hline & Tauriphila australis Hagen, 1867 & CE & Navás 1924 \\
\hline & $\begin{array}{l}\text { Tramea abdominalis } \\
\text { (Rambur, 1842) }\end{array}$ & CE & Pinto 2010 \\
\hline & Tramea calverti Muttkowski, 1910 & CE & $\begin{array}{l}\text { Nobre and Carvalho } \\
2014\end{array}$ \\
\hline & Tramea cophysa Hagen, 1867 & CE & $\begin{array}{l}\text { Nobre and Carvalho } \\
2014\end{array}$ \\
\hline & Uracis imbuta (Burmeister, 1839) & CE, PI & Costa and Santos 1997 \\
\hline \multirow[t]{2}{*}{ Calopterygidae } & Hetaerina rosea Selys, 1853 & CE & Navás 1924 \\
\hline & Mnesarete cupraea (Selys, 1853) & CE & $\begin{array}{l}\text { Carvalho and Bravo } \\
2014\end{array}$ \\
\hline \multirow[t]{12}{*}{ Coenagrionidae } & Acanthagrion gracile (Rambur, 1842) & CE & Navás 1924 \\
\hline & Argia cf. modesta Selys, 1865 & CE & $\begin{array}{l}\text { Nobre and Carvalho } \\
2014\end{array}$ \\
\hline & Argia hasemani Calvert, 1909 & CE & $\begin{array}{l}\text { Carvalho and Bravo } \\
2014\end{array}$ \\
\hline & Argia reclusa Selys, 1865 & CE & Navás 1924 \\
\hline & Enallagma novaehispaniae Calvert, 1907 & CE & $\begin{array}{l}\text { Nobre and Carvalho } \\
2014\end{array}$ \\
\hline & Ischnura capreolus (Hagen, 1861) & CE & Navás 1924 \\
\hline & Ischnura fluviatilis Selys, 1876 & CE & Pinto 2010 \\
\hline & Leptagrion dardanoi Santos, 1968 & CE & Santos 1968 \\
\hline & Neoneura sylvatica Hagen in Selys, 1886 & CE & $\begin{array}{l}\text { Nobre and Carvalho } \\
2014\end{array}$ \\
\hline & Oxyagrion chapadense Costa, 1978 & CE & $\begin{array}{l}\text { Carvalho and Bravo } \\
2014\end{array}$ \\
\hline & Telebasis corallina (Selys, 1876) & CE & $\begin{array}{l}\text { Nobre and Carvalho } \\
2014\end{array}$ \\
\hline & Telebasis filiola (Perty, 1834) & CE & $\begin{array}{l}\text { Nobre and Carvalho } \\
2014\end{array}$ \\
\hline Lestidae & Lestes forficula Rambur, 1842 & CE & Pinto 2010 \\
\hline Perilestidae & $\begin{array}{l}\text { Perilestes solutus Williamson \& Williamson, } \\
1924\end{array}$ & CE & Machado 2015 \\
\hline
\end{tabular}




\begin{tabular}{|c|c|c|c|}
\hline \multicolumn{4}{|l|}{ PLECOPTERA } \\
\hline Perlidae & Anacroneuria calori Duarte \& Lecci, 2016 & CE & Duarte and Lecci 2016 \\
\hline \multicolumn{4}{|l|}{ TRICHOPTERA } \\
\hline \multirow[t]{3}{*}{ Calamoceratidae } & Phylloicus abdominalis (Ulmer, 1905) & $\mathrm{CE}$ & Quinteiro et al. 2014 \\
\hline & Phylloicus bidigitatus Prather, 2003 & $\mathrm{CE}$ & Quinteiro et al. 2014 \\
\hline & Phylloicus obliquus Navás, 1931 & CE & Quinteiro et al. 2014 \\
\hline Helicopsychidae & Helicopsyche sp. & CE, PI & Quinteiro et al. 2014 \\
\hline \multirow[t]{4}{*}{ Hydropsychidae } & Leptonema pallidum Guérin, 1834 & $\mathrm{CE}$ & Costa et al. 2014 \\
\hline & Leptonema viridianum Navás, 1916 & CE & Costa et al. 2014 \\
\hline & Macrostemum hyalinum (Pictet, 1836) & $\mathrm{CE}$ & Franca et al. 2013 \\
\hline & Smicridea sp. & CE & Quinteiro et al. 2014 \\
\hline \multirow[t]{11}{*}{ Hydroptilidae } & $\begin{array}{l}\text { \#Betrichia nhundiaquara Souza, Santos \& } \\
\text { Takiya, } 2016\end{array}$ & $\mathrm{PI}$ & Souza et al. 2016b \\
\hline & $\begin{array}{l}\text { \#Flintiella harrisi Souza, Santos \& Takiya, } \\
2016\end{array}$ & $\mathrm{PI}$ & Souza et al. 2016a \\
\hline & $\begin{array}{l}\text { \#Hydroptila marighellai Souza, Santos \& } \\
\text { Takiya, } 2014\end{array}$ & $\mathrm{CE}$ & Souza et al. 2014a \\
\hline & $\begin{array}{l}\text { \#Hydroptila florestani Souza, Santos \& } \\
\text { Takiya, } 2014\end{array}$ & $\mathrm{PI}$ & Souza et al. 2014a \\
\hline & $\begin{array}{l}\text { \#Metrichia acuminata Santos, Takiya \& } \\
\text { Nessimian, } 2016\end{array}$ & CE & Santos et al. 2016a \\
\hline & $\begin{array}{l}\text { \#Metrichia rafaeli Santos, Takiya \& } \\
\text { Nessimian, } 2016\end{array}$ & CE & Santos et al. 2016a \\
\hline & $\begin{array}{l}\text { \#Metrichia ubajara Santos, Takiya \& } \\
\text { Nessimian, } 2016\end{array}$ & CE & Santos et al. 2016a \\
\hline & $\begin{array}{l}\text { \#Metrichia vulgaris Santos, Takiya \& } \\
\text { Nessimian, } 2016\end{array}$ & CE & Santos et al. 2016a \\
\hline & $\begin{array}{l}\text { \#Ochrotrichia caatinga Souza, Santos \& } \\
\text { Takiya, } 2014\end{array}$ & CE & Souza et al. 2014b \\
\hline & $\begin{array}{l}\text { \#Ochrotrichia limeirai Souza, Santos \& } \\
\text { Takiya, } 2014\end{array}$ & CE & Souza et al. 2014b \\
\hline & $\begin{array}{l}\text { \#Ochrotrichia patulosa (Wasmund \& } \\
\text { Holzenthal, 2007) }\end{array}$ & $\mathrm{CE}$ & Souza et al. $2014 b$ \\
\hline Leptoceridae & $\begin{array}{l}\text { \#Atanatolica nordestina Henriques-Oliveira } \\
\text { \& Santos, } 2014\end{array}$ & CE & $\begin{array}{l}\text { Henriques-Oliveira and } \\
\text { Santos } 2014\end{array}$ \\
\hline
\end{tabular}




\begin{tabular}{|c|c|c|c|}
\hline & Nectopsyche splendida (Navás, 1917) & PI & Quinteiro et al. 2014 \\
\hline & \#Oecetis connata Flint, 1974 & $\mathrm{PI}$ & $\begin{array}{l}\text { Henriques-Oliveira et } \\
\text { al. } 2014\end{array}$ \\
\hline & Oecetis excisa Ulmer, 1907 & CE & Quinteiro et al. 2014 \\
\hline & Oecetis inconspicua (Walker, 1852) & $\mathrm{PI}$ & Quinteiro et al. 2014 \\
\hline & Oecetis punctipennis (Ulmer, 1905) & CE & Quinteiro et al. 2014 \\
\hline Odontoceridae & Marilia sp. & CE & Quinteiro et al. 2014 \\
\hline Philopotamidae & Chimarra (Curgia) conica Flint, 1983 & $\mathrm{CE}$ & Flint 1998 \\
\hline
\end{tabular}

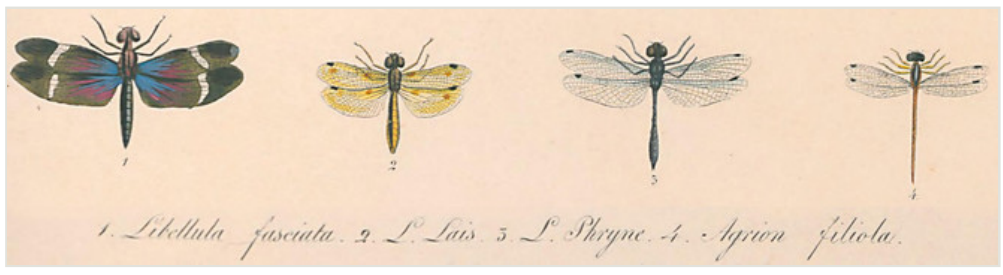

Figure 2.

Detail of plate 25 modified from Joseph Anton Maximilian Perty's work from 1834, with a water color illustration of the holotype of the small skimmer dragonfly Libellula phryne (Fig. 3, Odonata: Libellulidae; currently Nephepeltia phryne) described by him from "Provincia Piauhiensi", Brazil.

In this paper, we provide a preliminary checklist of selected taxa of aquatic insects from Ubajara and Sete Cidades National Parks in Northeastern Brazil (Fig. 1), an undersampled region in terms of biological inventories. Additionally, species richness was compared between the two National parks. As far as we know, this is the first published aquatic insect inventory of the selected National Parks.

\section{Materials and methods}

\section{Focal taxa of aquatic insects}

Identification of aquatic insects depended on the availability of specialists, therefore we focused on Ephemeroptera; suborders Nepomorpha and Gerromorpha of Hemiptera; families Elmidae, Epimetopidae, Hydrophilidae, and Torridincolidae of Coleoptera; Hemerodromiinae (aquatic Empididae) of Diptera; Odonata; Plecoptera; and Trichoptera. These focal taxa were identified at least in genus level, given that in some cases specieslevel identification was not possible because collected individuals were not adult males (immatures, subimagoes, or adult females), and, in other cases, because of the lack of comprehensive taxonomic revisions for particular genera. Higher-level classification followed the "Catálogo Taxonômico da Fauna do Brasil" (Lecci and Froehlich 2016, Monné 
et al. 2016, Pinto 2016, Rafael and Câmara 2016, Salles and Boldrini 2016, Santos et al. 2016).

\section{Study areas}

Material examined in this paper were obtained during two collecting expeditions to the study areas, from April $18^{\text {th }}$ to $21^{\text {st }}$ of 2012 and February $7^{\text {th }}$ to $13^{\text {th }}$ of 2013 at Parque Nacional de Sete Cidades (PNSC, Piracuruca municipality, PI) and from Abril $21^{\text {st }}$ to $25^{\text {th }}$ of 2012 and February $13^{\text {th }}$ to $20^{\text {th }}$ of 2013 at Parque Nacional de Ubajara (PNU, Ubajara municipality, CE). Although both parks are included in the Caatinga Domain (Fig. 1), areas sampled within these parks seem to represent islands dominated by Cerrado (PNSC) or montane humid forest (PNU). The two parks are distant by approximately $110 \mathrm{~km}$.

Sampling localities within these parks were in or very close (less than $100 \mathrm{~m}$ ) to bodies of water and are listed in Table 3. Bodies of water ranged from lentic and lotic systems and some streams sampled during the 2012 expedition to PNSC were completely dry in 2013 (see Figs 3, 4).

Table 3.

Sampling localities in Parque Nacional de Ubajara (PNU) and Parque Nacional de Sete Cidades (PNSC).

\begin{tabular}{|c|c|c|c|}
\hline Code & Locality & Coordinates & Altitude (m) \\
\hline PNU-01 & PNU, Trilha Samambaia, Rio Gameleira (Fig. 5) & $3^{\circ} 50^{\prime} 25^{\prime \prime S}, 40^{\circ} 54^{\prime} 19^{\prime \prime} \mathrm{W}$ & 874 \\
\hline PNU-02 & PNU, Trilha Araticum, Rio das Minas (Fig. 6) & $3^{\circ} 50^{\prime} 3^{\prime \prime} \mathrm{S}, 40^{\circ} 54^{\prime} 18^{\prime \prime} \mathrm{W}$ & 524 \\
\hline PNU-03 & PNU, Portão Neblina & $3^{\circ} 50^{\prime} 18^{\prime \prime S}, 40^{\circ} 53^{\prime} 54^{\prime \prime W}$ & 849 \\
\hline PNU-04 & PNU, Trilha Araticum, Rio Cafundó (Fig. 7) & $3^{\circ} 50^{\prime} 12^{\prime \prime} \mathrm{S}, 40^{\circ} 54^{\prime} 31^{\prime \prime W}$ & 753 \\
\hline PNU-05 & $\begin{array}{l}\text { PNU, Trilha Samambaia, Mirante da cachoeira do } \\
\text { Gameleira }\end{array}$ & $3^{\circ} 50^{\prime} 21 " \mathrm{~S}, 40^{\circ} 54^{\prime} 23^{\prime \prime} \mathrm{W}$ & 880 \\
\hline PNU-06 & $\begin{array}{l}\text { PNU, Trilha Araticum, Rio das Minas na altura da } \\
\text { trilha do teleférico }\end{array}$ & $3^{\circ} 49^{\prime} 58 " \mathrm{~S}, 40^{\circ} 53^{\prime} 53^{\prime \prime} \mathrm{W}$ & 420 \\
\hline PNU-07 & PNU, Cachoeira do Cafundó (Fig. 8) & $3^{\circ} 50^{\prime} 12 " \mathrm{~S}, 40^{\circ} 54^{\prime} 35^{\prime \prime} \mathrm{W}$ & 783 \\
\hline PNU-08 & PNU, Rio Cafundó, pouco acima da cachoeira & $3^{\circ} 50^{\prime} 13^{\prime \prime} \mathrm{S}, 40^{\circ} 54^{\prime} 35^{\prime \prime} \mathrm{W}$ & 795 \\
\hline PNU-09 & $\begin{array}{l}\text { PNU, Trilha Araticum, Rio da Minas abaixo do } \\
\text { teleférico }\end{array}$ & $3^{\circ} 49^{\prime} 43.3^{\prime \prime} \mathrm{S}, 40^{\circ} 53^{\prime} 51.5^{\prime \prime} \mathrm{W}$ & 395 \\
\hline PNU-10 & PNU, Ponte sobre Rio Miranda & $3^{\circ} 50^{\prime} 7.4^{\prime \prime} \mathrm{S}, 40^{\circ} 54^{\prime} 47.5^{\prime \prime} \mathrm{W}$ & 792 \\
\hline PNU-11 & PNU, Rio das Minas, próximo ao Portão Araticum & $3^{\circ} 49^{\prime} 32.6^{\prime \prime} \mathrm{S}, 40^{\circ} 53^{\prime} 32.8^{\prime \prime} \mathrm{W}$ & 328 \\
\hline PNU-12 & PNU, Trilha das Samambaias, brejo & $3^{\circ} 50^{\prime} 25.8^{\prime \prime S}, 40^{\circ} 54^{\prime} 28.4^{\prime \prime} \mathrm{W}$ & 829 \\
\hline PNU-13 & PNU, Mijo da Velha & $3^{\circ} 50^{\prime} 16.7^{\prime \prime S}, 40^{\circ} 54^{\prime} 34.2^{\prime \prime} \mathrm{W}$ & 768 \\
\hline
\end{tabular}




\begin{tabular}{|l|l|l|l|}
\hline PNSC-01 & PNSC, Riacho da Bananeira (Figs 9, 10) & $4^{\circ} 5^{\prime} 59^{\prime \prime} \mathrm{S}, 41^{\circ} 40^{\prime} 48^{\prime \prime} \mathrm{W}$ & 189 \\
\hline PNSC-02 & PNSC, Cachoeira do Riachão (Figs 3, 11) & $4^{\circ} 6^{\prime} 28^{\prime \prime} \mathrm{S}, 41^{\circ} 40^{\prime} 13^{\prime \prime} \mathrm{W}$ & 171 \\
\hline PNSC-03 & PNSC, Alojamento & $4^{\circ} 5^{\prime} 57 " \mathrm{~S}, 41^{\circ} 42^{\prime} 34^{\prime \prime} \mathrm{W}$ & 193 \\
\hline PNSC-04 & PNSC, Riacho da Piedade (Fig. 4) & $4^{\circ} 6^{\prime} 34^{\prime \prime S}, 41^{\circ} 43^{\prime} 39^{\prime \prime} \mathrm{W}$ & 169 \\
\hline PNSC-05 & PNSC, Centro de visitantes & $4^{\circ} 6^{\prime} 20^{\prime \prime} \mathrm{S}, 41^{\circ} 41^{\prime} 52^{\prime \prime} \mathrm{W}$ & 202 \\
\hline PNSC-06 & PNSC, Olho d'água Piscina do Bacuri & $4^{\circ} 6^{\prime} 1.2^{\prime \prime S}, 41^{\circ} 42^{\prime} 38.8 " \mathrm{~W}$ & 171 \\
\hline PNSC-07 & PNSC, Olho d'água dos Milagres (Fig. 12) & $4^{\circ} 5^{\prime} 31.8^{\prime \prime S}, 41^{\circ} 40^{\prime} 48.2^{\prime \prime} \mathrm{W}$ & 180 \\
\hline PNSC-08 & PNSC, Poço do Bananeira (Fig. 13) & $4^{\circ} 5^{\prime} 55.8^{\prime \prime S}, 41^{\circ} 40^{\prime} 33.8^{\prime \prime} \mathrm{W}$ & 158 \\
\hline PNSC-09 & PNSC, trilha para Poço do Bananeira & $4^{\circ} 5^{\prime} 56.6^{\prime \prime S}, 41^{\circ} 40^{\prime} 32.3^{\prime \prime} \mathrm{W}$ & 162 \\
\hline
\end{tabular}
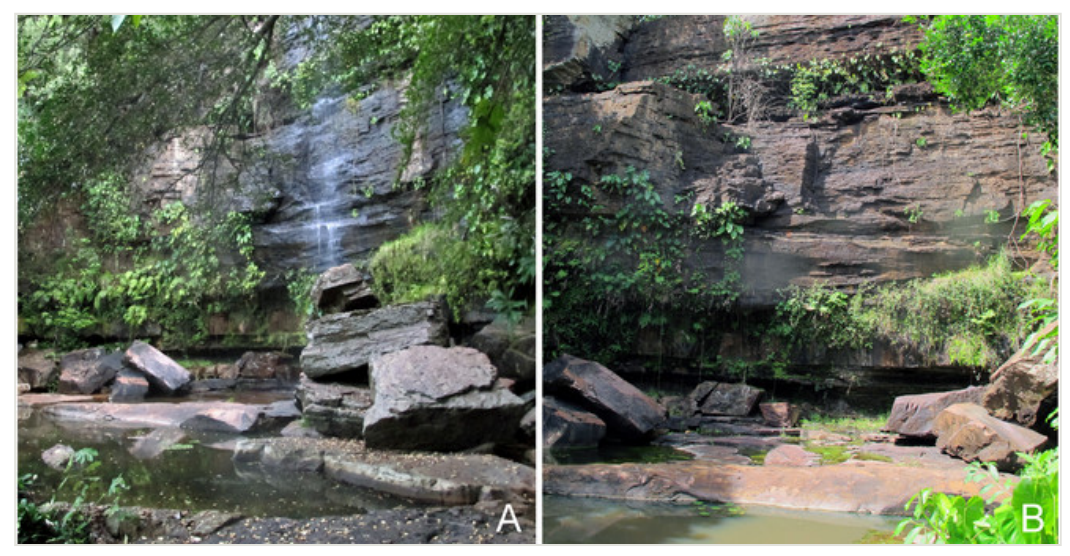

Figure 3.

Cachoeira do Riachão (PNSC-02) at Parque Nacional de Sete Cidades, PI, Brazil. (A) April 2012. (B) February 2013.

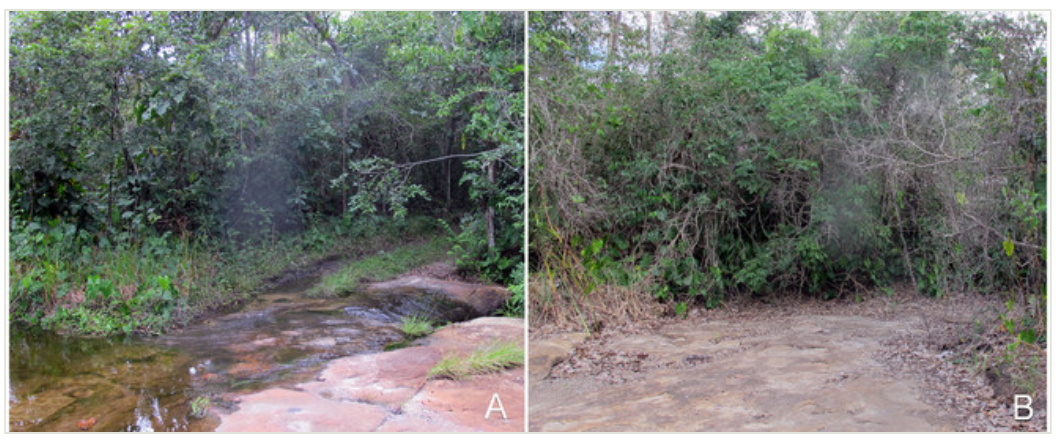

Figure 4.

Riacho da Piedade (PNSC-04) at Parque Nacional de Sete Cidades, PI, Brazil. (A) April 2012. (B) February 2013. 


\section{Sampling methods}

The following insects traps were used: 6-meter intercept Malaise traps (Figs 5, 11, Gressitt and Gressitt 1962), suspended intercept traps (Fig. 14, Rafael and Gorayeb 1982), light traps using a white sheet (Fig. 15) and Pennsylvania traps (Fig. 12, Frost 1957), yellow pan traps (Fig. 16), in addition to manual collecting using forceps or entomological nets (Fig. 10). Besides individuals collected during both expeditions, material was also collected by Malaise traps that were left in both parks with samples removed every month between expeditions. Material collected was divided and deposited in the following institutions: Coleção Entomológica Prof. José Alfredo Pinheiro Dutra, Departamento de Zoologia, Universidade Federal do Rio de Janeiro, Rio de Janeiro (DZRJ); Coleção Zoológica do Maranhão, Universidade Estadual do Maranhão, Caxias (CZMA); and Coleção de Invertebrados, Instituto Nacional de Pesquisas da Amazônia, Manaus (INPA).

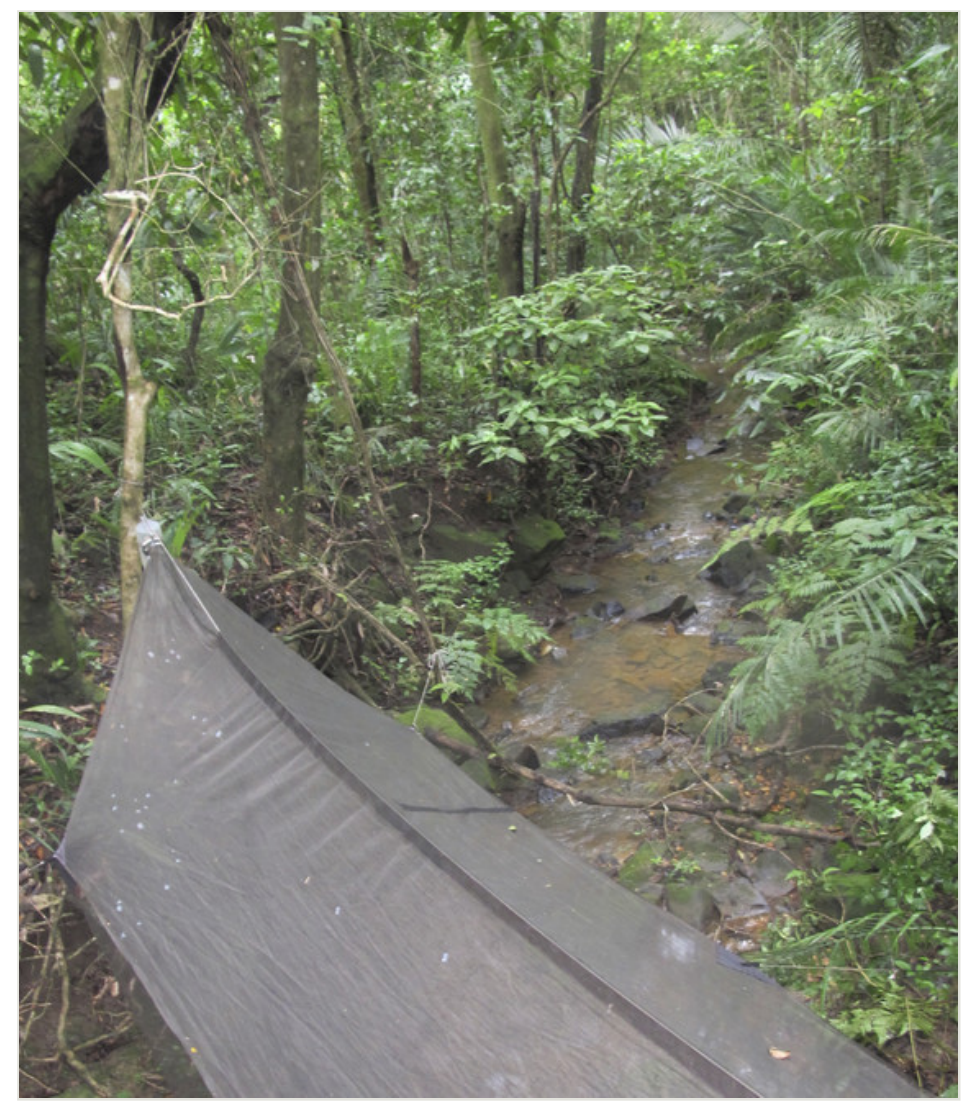

Figure 5.

6-meter Malaise flight intercept trap over Rio Gameleira crossing Trilha Samambaia (PNU-01) at Parque Nacional de Ubajara, CE, Brazil. 


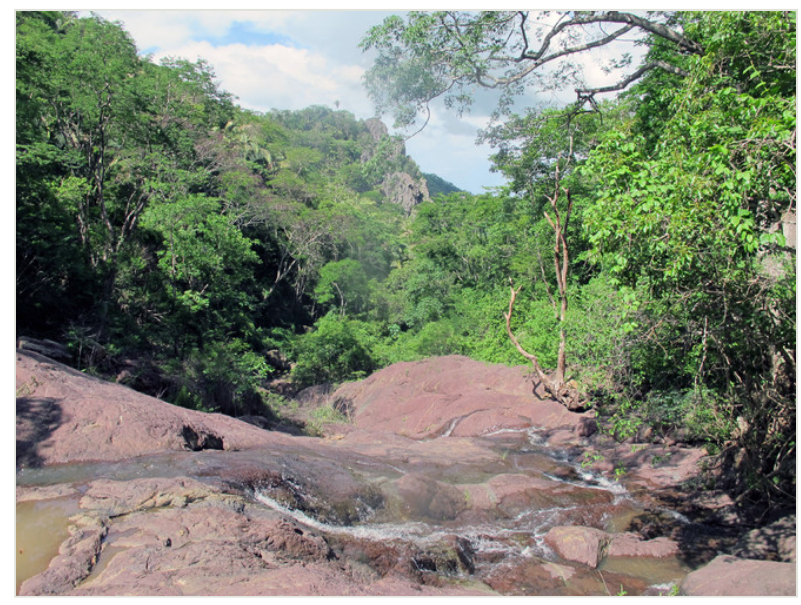

Figure 6.

Rio das Minas crossing Trilha Araticum (PNU-02) at Parque Nacional de Ubajara, CE, Brazil.

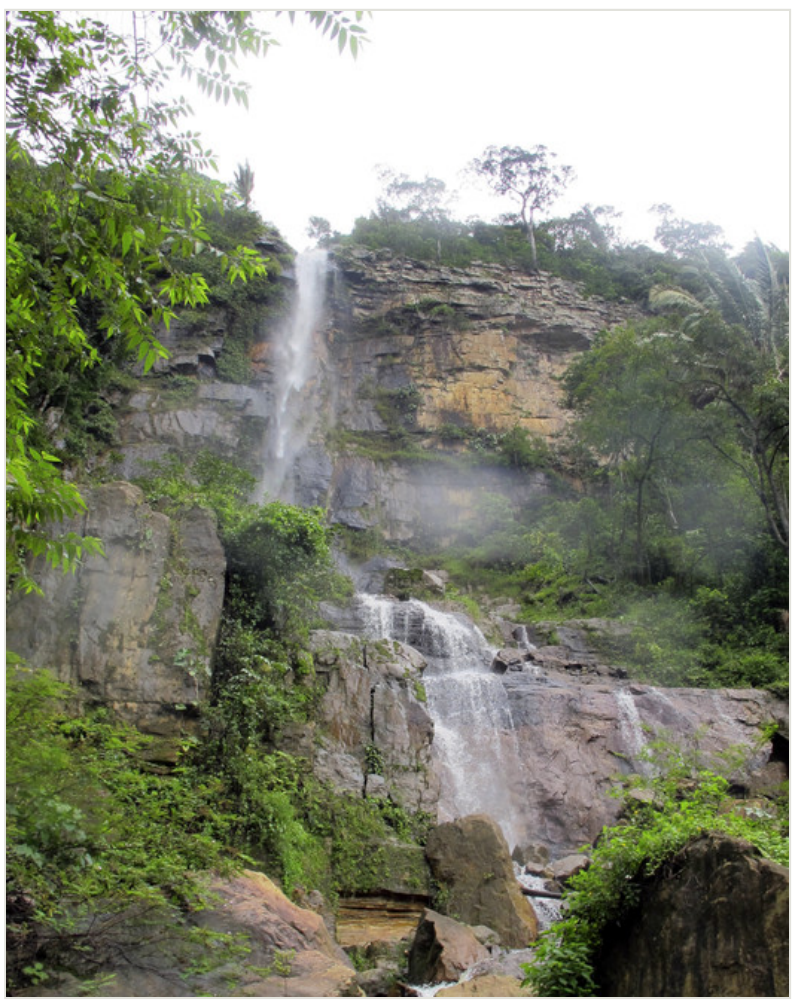

Figure 7.

Rio Cafundó crossing Trilha Araticum (PNU-04) at Parque Nacional de Ubajara, CE, Brazil. 


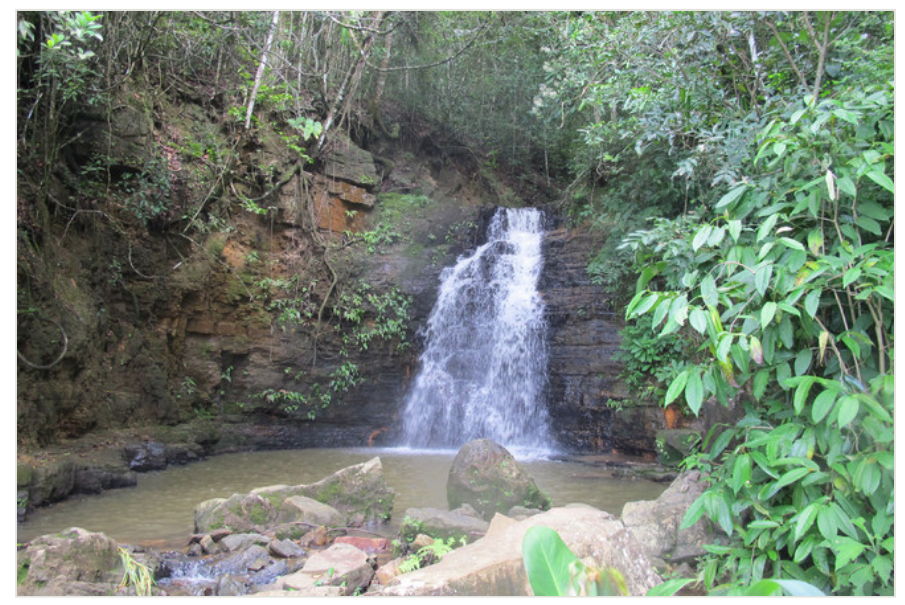

Figure 8.

Cachoeira do Cafundó (PNU-07) at Parque Nacional de Ubajara, CE, Brazil.

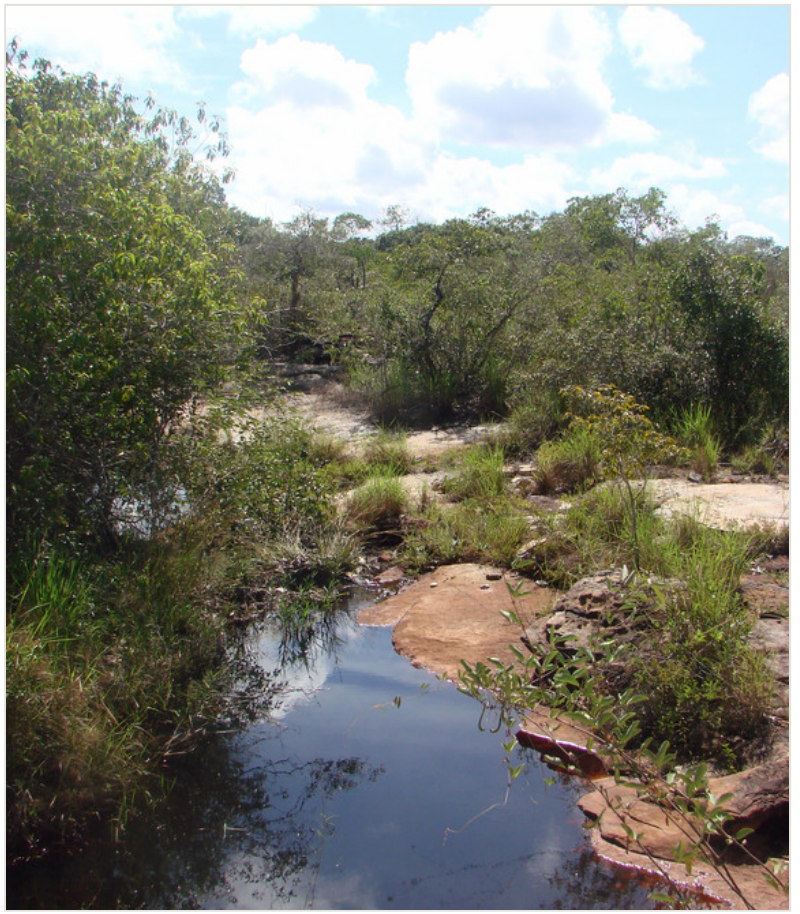

Figure 9.

Riacho da Bananeira (PNSC-01) at Parque Nacional de Sete Cidades, PI, Brazil. Photo by A. Somavilla. 


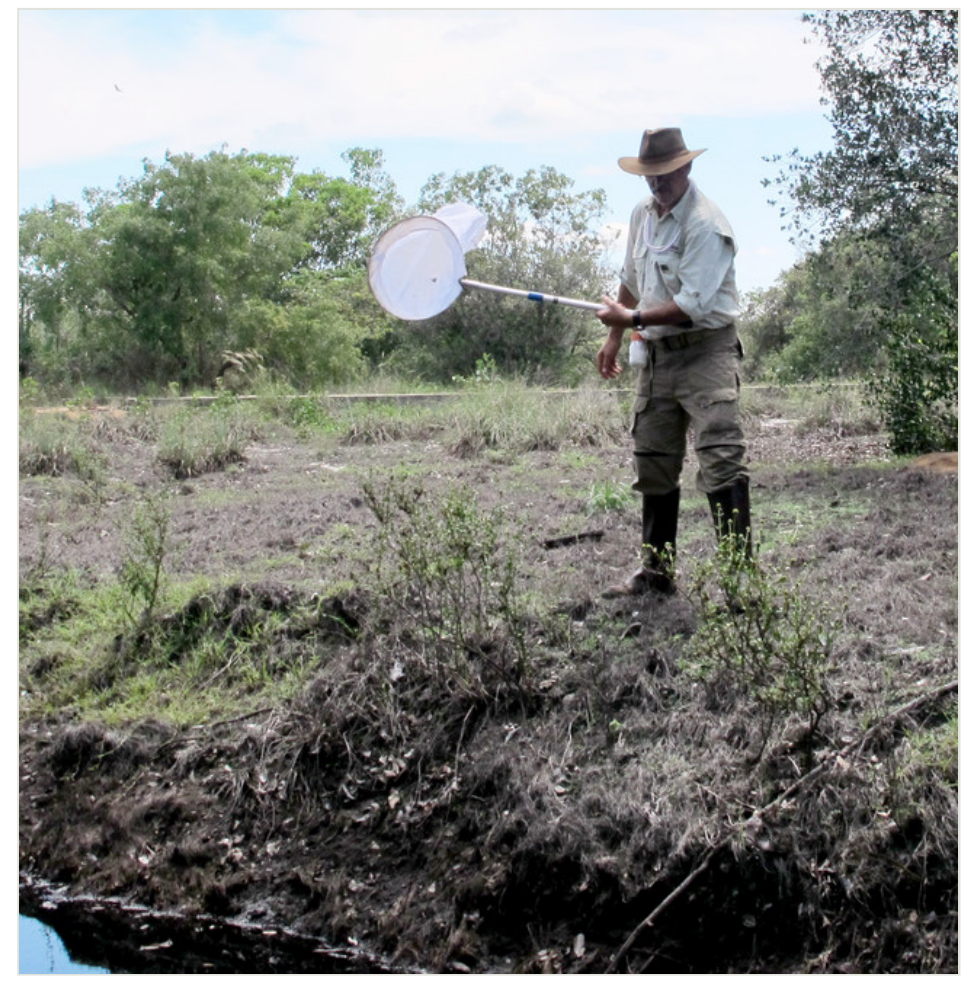

Figure 10.

Manual collecting with entomological net near border of Riacho do Bananeira (PNSC-01), Parque Nacional de Sete Cidades, PI, Brazil.

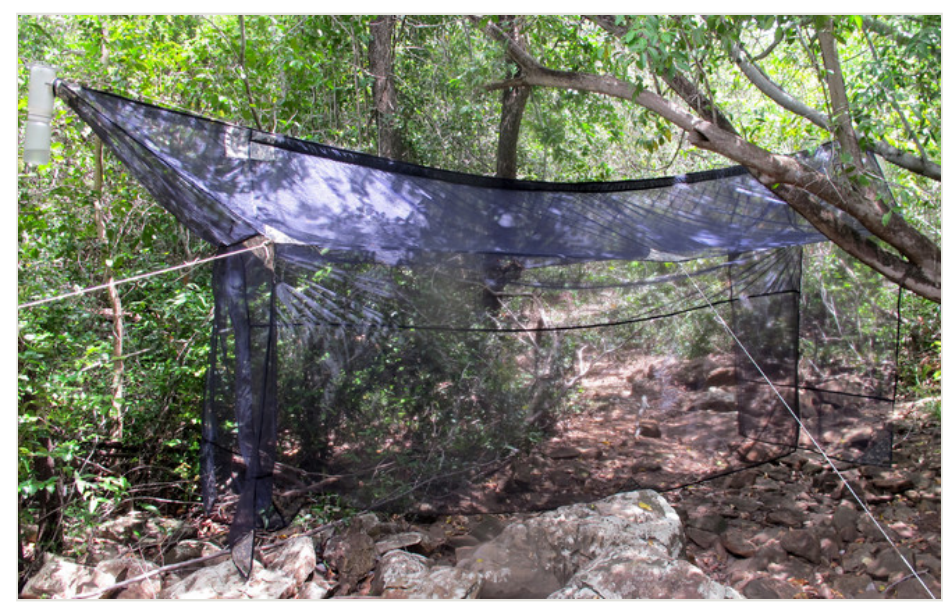

Figure 11.

6-meter Malaise flight intercept trap over seasonally dried stream below Cachoeira do Riachão (PNSC-02) at Parque Nacional de Sete Cidades, PI, Brazil. 


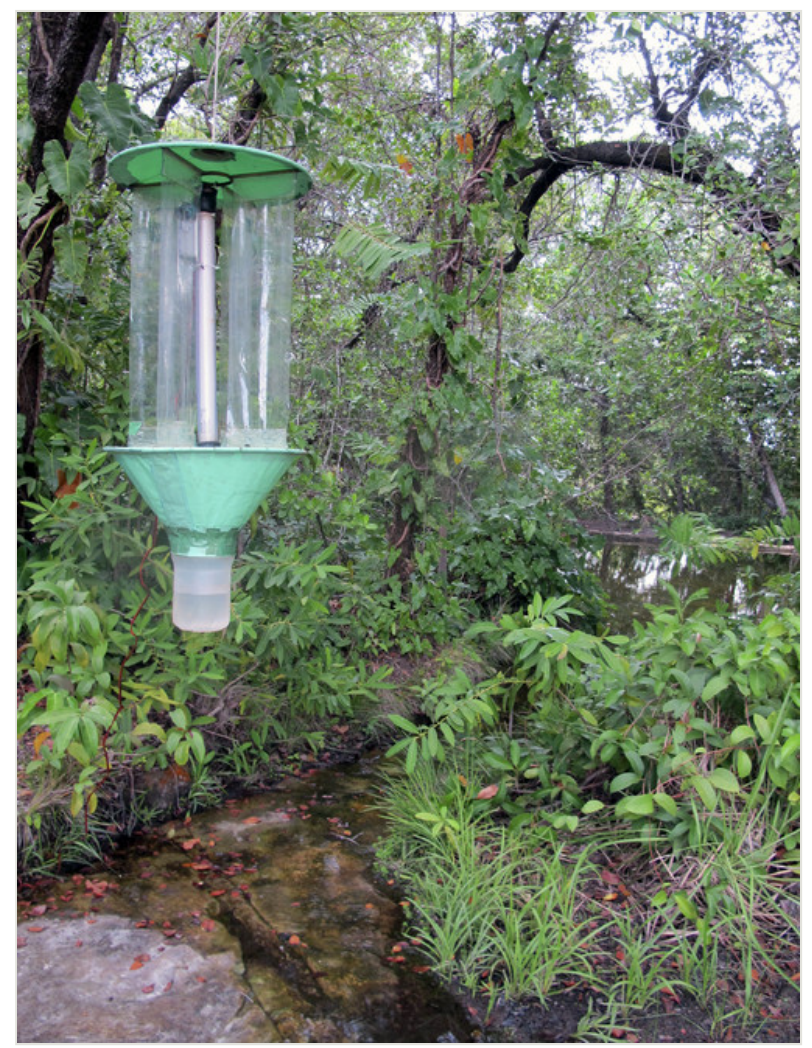

Figure 12.

Pensylvania light trap near Olho d'água dos Milagres (PNSC-07) at Parque Nacional de Sete Cidades, PI, Brazil.

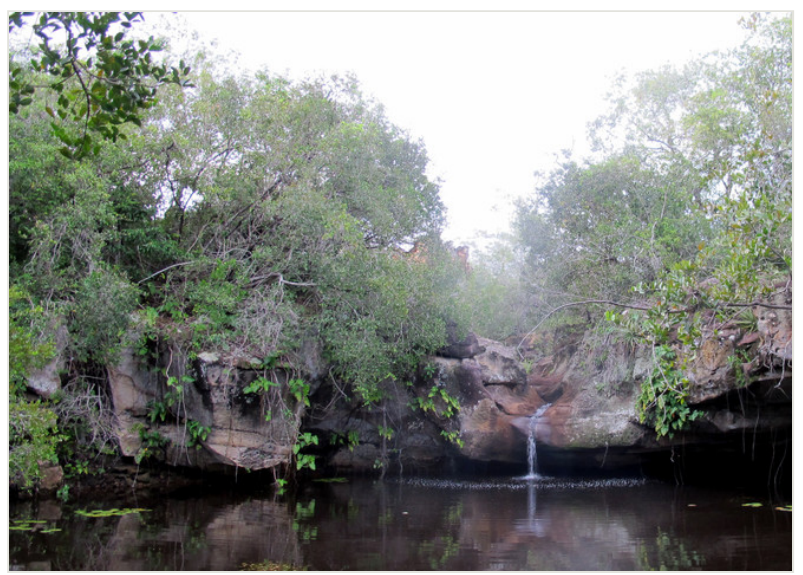

Figure 13.

Poço do Bananeira (PNSC-08) at Parque Nacional de Sete Cidades, PI, Brazil. 


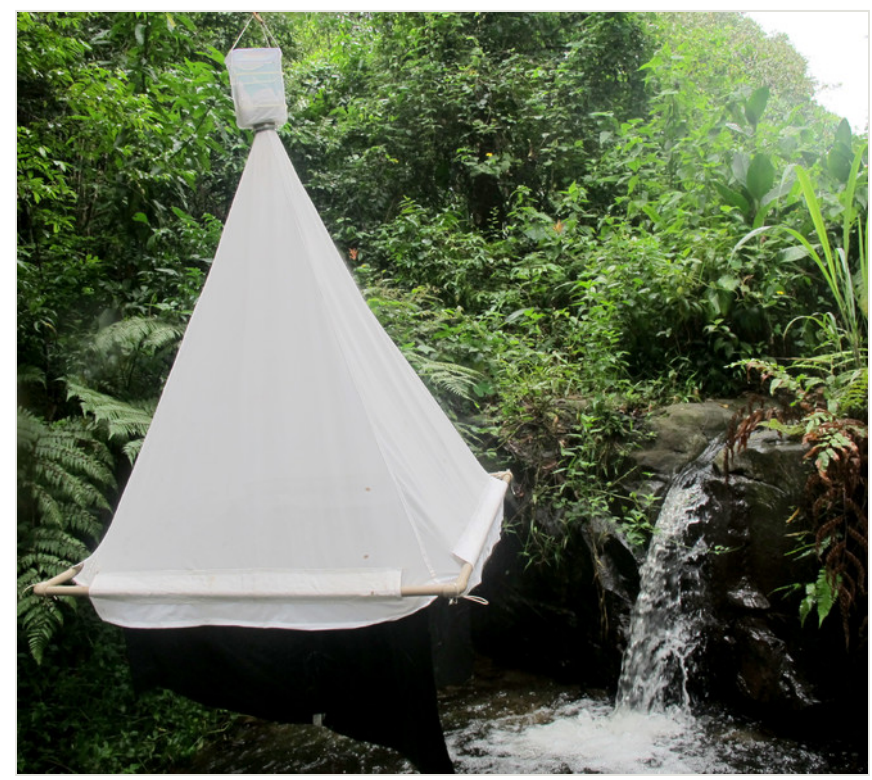

Figure 14.

Suspended intercept trap over stream at Parque Nacional de Ubajara, CE, Brazil.

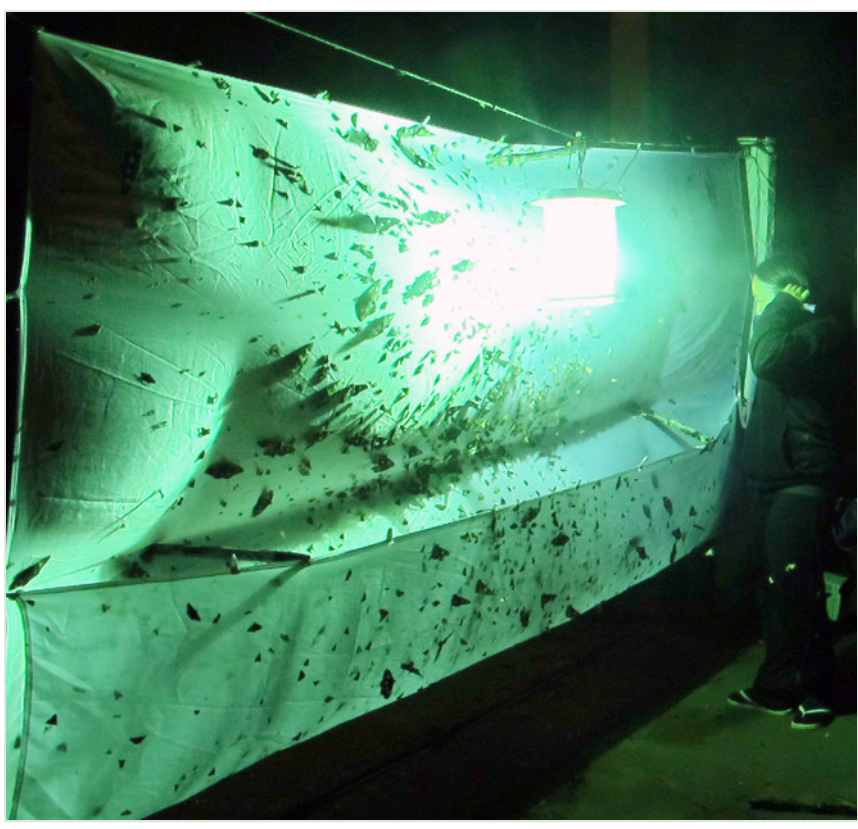

Figure 15.

Manual collecting using white-sheet light trap at Parque Nacional de Ubajara, CE, Brazil. 


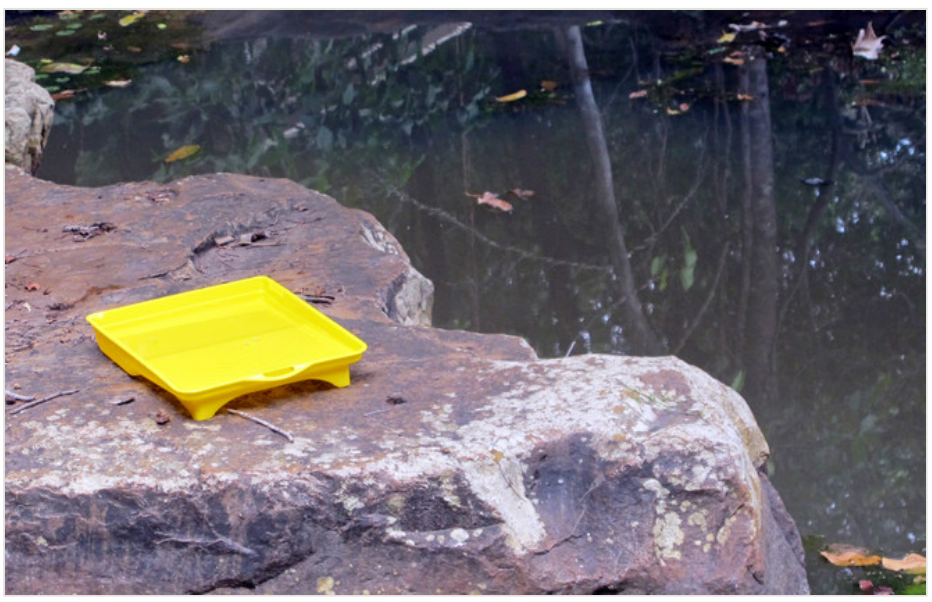

Figure 16.

Yellow pan trap (YPT) near border of stream at Parque Nacional de Sete Cidades, PI, Brazil.

\section{Data analyses}

Species complementarity index for the two National parks were calculated with Cjk $=$ Ujk/ Sjk, where Ujk is the number of unique species in both sites and Sjk total richness in both sites combined (Colwell and Coddington 1994).

During the expeditions 27 samples (14 at PNSC and 13 at PNU) of Pennsylvania light traps placed over streams were collected. Each sample was the result of an approximately 6-8 hour effort of $15 \mathrm{~W}$ fluorescent or UV light turned on at dusk. All individuals of Ephemeroptera, Plecoptera, Trichoptera, and Hydrophilidae (Coleoptera) were identified or morphotyped and counted. Adult females or subimagoes impossible to be identified into species level were treated as conspecific as males identified of the same genus on the sample (6 morphotypes from PNSC and 8 morphotypes from PNU of Ephemeroptera and Trichoptera). However, in cases where more than one species of the same genus was identified from the same sample (Helicopsyche and Oxyethira from PNU and Chimarra, Neotrichia, Smicridea, and Oxyethira from PNSC), the number of female individuals were equally divided among different species.

Quantitative samples of Pennsylvania light traps totalled 227 individuals of 23 species from PNU and 511 individuals of 54 species from PNSC of Ephemeroptera, Plecoptera, Trichoptera and Hydrophilidae (Coleoptera). Quantitative data served for comparisons of species richness between the two National Parks based on a rarefaction curve by individuals calculated in PAST 3.0 (Hammer et al. 2001). 


\section{Data resources}

Data for species checklists of Parque Nacional de Ubajara (PNU) e Parque Nacional de Sete Cidades (PNSC)

Approximately 7,000 individuals of focal taxa were identified, being 5,472 from PNU and 1,539 from PNSC (Fig. 17).

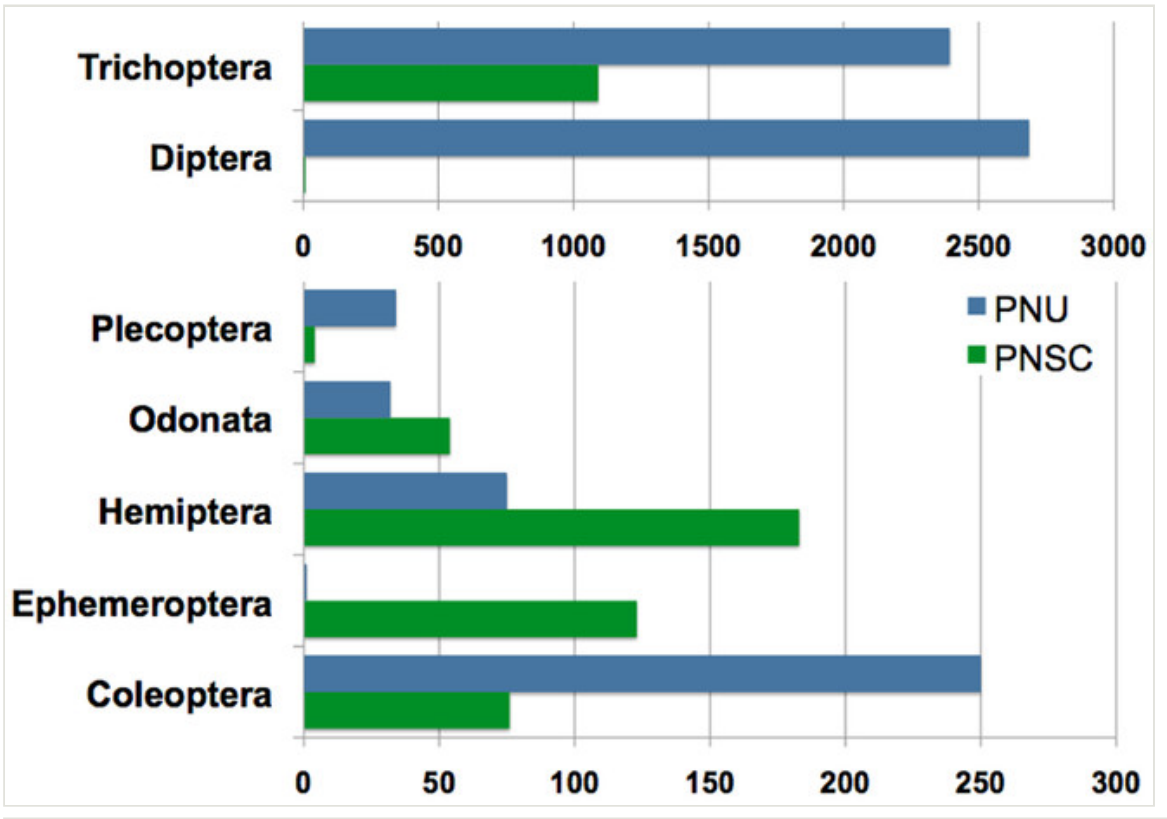

Figure 17.

Number of individuals of aquatic insects identified from Ubajara (PNU, blue) and Sete Cidades (PNSC, green) National Parks.

These individuals were identified into 96 species from PNU and 112 species from PNSC, while 20 of these species were found in both parks (Fig. 18). Species complementarity between both parks was of $89 \%$.

The number of species per insect order in PNU and PNSC, respectively were: Coleoptera, 16 and 20; Diptera 10 and 1; Ephemeroptera, 1 and 9; Hemiptera, 9 and 20; Odonata, 21 and 21; Plecoptera, 1 and 1; and Trichoptera, 38 and 40. Species checklists for both National Parks are given below. Species marked with an asterisk $\left({ }^{\star}\right)$ were found in both National Parks and distributional records (country or state) marked with and exclamation mark (!) are new records published herein. Species described or first taxa records based on material collected during this project are cited under Notes. 


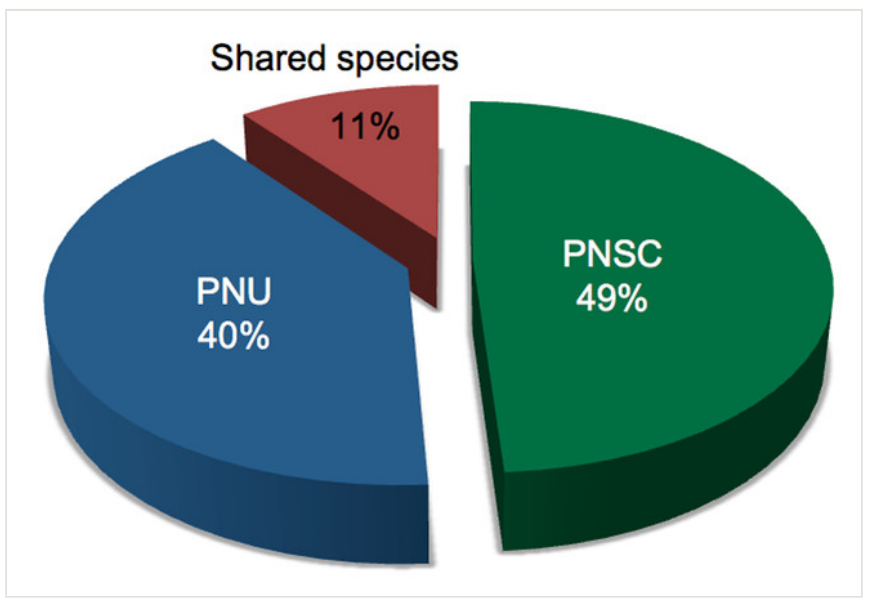

Figure 18.

Percentage of total species of aquatic insects found only at Ubajara (PNU, blue), only at Sete Cidades (PNSC, green), and in both National Parks (red).

\section{Aquatic insects from Parque Nacional de Ubajara (PNU)}

\section{Order Coleoptera}

\section{Family Elmidae}

Notes: New family record for CE.

\section{Genus Cylloepus Erichson, 1847}

Notes: New genus record for CE.

\section{Cylloepus sp. 1}

Material

a. country: Brazil; stateProvince: Ceará; municipality: Ubajara; locality: Parque Nacional de Ubajara, Trilha Araticum, Rio das Minas; maximumElevationInMeters: 524; verbatimCoordinates: $3^{\circ} 50$ '3"S, 4054'18"W; samplingProtocol: Manual; verbatimEventDate: 18.ii.13; individualCount: 1; lifeStage: adult; recordedBy: Santos, A.P.M. | Takiya, D.M.; identifiedBy: Brunno H. L. Sampaio; institutionCode: DZRJ; basisOfRecord: PreservedSpecimen

\section{Genus Macrelmis Motschulsky, 1859}

Notes: New genus record for CE. 


\section{Macrelmis sp. 1}

\section{Material}

a. country: Brazil; stateProvince: Ceará; municipality: Ubajara; locality: Parque Nacional de Ubajara, Trilha Araticum, Rio das Minas; maximumElevationInMeters: 524; verbatimCoordinates: $3^{\circ} 50^{\prime} 3^{\prime \prime S}$, 4054'18"W; samplingProtocol: Manual; verbatimEventDate: 18.ii.13; individualCount: 2; lifeStage: immature; recordedBy: Santos, A.P.M. | Takiya, D.M.; identifiedBy: Brunno H. L. Sampaio; institutionCode: DZRJ; basisOfRecord: PreservedSpecimen

\section{Genus Microcylloepus Hinton, 1935}

Notes: New genus record for CE.

\section{Microcylloepus sp. 1}

\section{Materials}

a. country: Brazil; stateProvince: Ceará; municipality: Ubajara; locality: Parque Nacional de Ubajara, Trilha Araticum, Rio das Minas; maximumElevationInMeters: 524; verbatimCoordinates: $3^{\circ} 50^{\prime} 3$ "S, $40^{\circ} 54^{\prime} 18$ "W; samplingProtocol: Manual; verbatimEventDate: 17.ii.13; individualCount: 1; lifeStage: immature; recordedBy: Santos, A.P.M. | Takiya, D.M.; identifiedBy: Brunno H. L. Sampaio; institutionCode: DZRJ; basisOfRecord: PreservedSpecimen

b. country: Brazil; stateProvince: Ceará; municipality: Ubajara; locality: Parque Nacional de Ubajara, Trilha Araticum, Rio das Minas; maximumElevationInMeters: 524; verbatimCoordinates: $3^{\circ} 50^{\prime} 3$ "S, $40^{\circ} 54^{\prime} 18^{\prime \prime} \mathrm{W}$; samplingProtocol: Manual; verbatimEventDate: 18.ii.13; individualCount: 5; lifeStage: adult; recordedBy: Santos, A.P.M. | Takiya, D.M.; identifiedBy: Brunno H. L. Sampaio; institutionCode: DZRJ; basisOfRecord: PreservedSpecimen

c. country: Brazil; stateProvince: Ceará; municipality: Ubajara; locality: Parque Nacional de Ubajara, Trilha Araticum, Rio das Minas; maximumElevationInMeters: 524; verbatimCoordinates: $3^{\circ} 50^{\prime} 3$ "S, 4054'18"W; sampling Protocol: Manual; verbatimEventDate: 23.iv.12; individualCount: 1; lifeStage: immature; recordedBy: Takiya, D.M.; identifiedBy: Brunno H. L. Sampaio; institutionCode: DZRJ; basisOfRecord: PreservedSpecimen

d. country: Brazil; stateProvince: Ceará; municipality: Ubajara; locality: Parque Nacional de Ubajara, Trilha Araticum, Rio das Minas; maximumElevationInMeters: 524; verbatimCoordinates: $3^{\circ} 50^{\prime} 3$ "S, $40^{\circ} 54^{\prime} 18 " \mathrm{~W}$; samplingProtocol: Manual; verbatimEventDate: 23.iv.12; individualCount: 2; lifeStage: adult; recordedBy: Takiya, D.M.; identifiedBy: Brunno H. L. Sampaio; institutionCode: DZRJ; basisOfRecord: PreservedSpecimen

\section{Family Epimetopidae}

Notes: New family record for CE. 
Genus Epimetopus Lacordaire, 1854

Notes: New genus record for CE.

\section{Epimetopus sp. 1}

Material

a. country: Brazil; stateProvince: Ceará; municipality: Ubajara; locality: Parque Nacional de Ubajara, Cachoeira do Cafundó; maximumElevationInMeters: 783; verbatimCoordinates: $3^{\circ} 50^{\prime} 12 " \mathrm{~S}, 40^{\circ} 54^{\prime} 35^{\prime \prime} \mathrm{W}$; samplingProtocol: Pennsylvania light trap; verbatimEventDate: 15.ii.13; individualCount: 1; sex: male; lifeStage: adult; recordedBy: Santos, A.P.M. | Takiya, D.M.; identifiedBy: Bruno Clarkson; institutionCode: DZRJ; basisOfRecord: PreservedSpecimen

Notes: Undescribed species in the lanceolatus group.

\section{Family Hydrophilidae}

\section{Genus Australocyon Hansen, 1990}

Notes: New genus record for CE.

\section{Australocyon sp. 1}

\section{Material}

a. country: Brazil; stateProvince: Ceará; municipality: Ubajara; locality: Parque Nacional de Ubajara, Cachoeira do Cafundó; maximumElevationInMeters: 783; verbatimCoordinates: $3^{\circ} 50^{\prime} 12^{\prime \prime} \mathrm{S}, 40^{\circ} 54^{\prime} 35 " \mathrm{~W}$; samplingProtocol: Pennsylvania light trap; verbatimEventDate: 24.iv.12; individualCount: 1; sex: male; lifeStage: adult; recordedBy: Takiya, D.M.; identifiedBy: Bruno Clarkson; institutionCode: DZRJ; basisOfRecord: PreservedSpecimen

Notes: Undescribed species.

\section{Genus Chasmogenus Sharp, 1882}

Notes: New genus record for CE.

\section{Chasmogenus (Chasmogenus) sp. $1^{*}$}

\section{Material}

a. $\quad$ country: Brazil; stateProvince: Ceará; municipality: Ubajara; locality: Parque Nacional de Ubajara, Trilha Araticum, Rio das Minas na altura da trilha do teleférico; maximumElevationInMeters: 420; verbatimCoordinates: 349'58"S, 4053'53"W; samplingProtocol: Malaise intercept trap; verbatimEventDate: 14.ii.13; individualCount: 4; lifeStage: adult; recordedBy: Santos, A.P.M. | Takiya, D.M.; identifiedBy: Bruno Clarkson; institutionCode: DZRJ; basisOfRecord: PreservedSpecimen 


\section{Genus Enochrus Thomson, 1859}

Notes: New genus record for CE.

\section{Enochrus (Methydrus) melanthus Orchymont, 1943}

\section{Materials}

a. country: Brazil; stateProvince: Ceará; municipality: Ubajara; locality: Parque Nacional de Ubajara, Trilha Araticum, Rio das Minas na altura da trilha do teleférico; maximumElevationInMeters: 420; verbatimCoordinates: 349'58"S, 4053'53"W; samplingProtocol: Malaise intercept trap; verbatimEventDate: 14.ii.13; individualCount: 9; lifeStage: adult; recordedBy: Santos, A.P.M. | Takiya, D.M.; identifiedBy: Bruno Clarkson; institutionCode: DZRJ; basisOfRecord: PreservedSpecimen

b. country: Brazil; stateProvince: Ceará; municipality: Ubajara; locality: Parque Nacional de Ubajara, Portão Neblina; maximumElevationInMeters: 849; verbatimCoordinates: $3^{\circ}$ 50'18"S, 4053'54"W; samplingProtocol: Malaise intercept trap; verbatimEventDate: 14.ii.13; individualCount: 1; sex: female; lifeStage: adult; recordedBy: Santos, A.P.M. | Takiya, D.M.; identifiedBy: Bruno Clarkson; institutionCode: DZRJ; basisOfRecord: PreservedSpecimen

c. country: Brazil; stateProvince: Ceará; municipality: Ubajara; locality: Parque Nacional de Ubajara, Rio Cafundó, pouco acima da cachoeira; maximumElevationInMeters: 795; verbatimCoordinates: 350'13"S, 4054'35"W; samplingProtocol: Malaise intercept trap; verbatimEventDate: 13.ii.13; individualCount: 1; sex: male; lifeStage: adult; recordedBy: Santos, A.P.M. | Takiya, D.M.; identifiedBy: Bruno Clarkson; institutionCode: DZRJ; basisOfRecord: PreservedSpecimen

Distribution: Brazil: CE!, PE, MS, SP.

Notes: New species record for CE.

\section{Genus Helochares Mulsant, 1844}

Notes: New genus record for CE.

\section{Helochares (Helochares) sp. 1*}

\section{Material}

a. country: Brazil; stateProvince: Ceará; municipality: Ubajara; locality: Parque Nacional de Ubajara, Trilha Araticum, Rio das Minas na altura da trilha do teleférico; maximumElevationInMeters: 420; verbatimCoordinates: $3^{\circ} 49^{\prime} 58$ "S, 4053'53"W; samplingProtocol: Manual; verbatimEventDate: 19.ii.13; individualCount: 1; lifeStage: immature; recordedBy: Santos, A.P.M. | Takiya, D.M.; identifiedBy: Bruno Clarkson; institutionCode: DZRJ; basisOfRecord: PreservedSpecimen 


\section{Genus Hemiosus Sharp, 1882}

\section{Hemiosus varidius Orchymont, 1940}

\section{Material}

a. country: Brazil; stateProvince: Ceará; municipality: Ubajara; locality: Parque Nacional de Ubajara, Trilha Araticum, Rio da Minas abaixo do teleférico; maximumElevationInMeters: 395; verbatimCoordinates: $3^{\circ} 49^{\prime} 43.3^{\prime \prime S}, 40^{\circ} 53^{\prime} 51.5^{\prime \prime} \mathrm{W}$; samplingProtocol: Manual; verbatimEventDate: 14.ii.13; individualCount: 1; sex: female; lifeStage: adult; recordedBy: Santos, A.P.M. | Takiya, D.M.; identifiedBy: Bruno Clarkson; institutionCode: DZRJ; basisOfRecord: PreservedSpecimen

Distribution: Brazil: CE!, PE.

Notes: New species record for CE.

\section{Genus Oocyclus Sharp, 1882}

Notes: New genus record for CE.

\section{Oocyclus schubarti* Orchymont, 1940}

\section{Materials}

a. country: Brazil; stateProvince: Ceará; municipality: Ubajara; locality: Parque Nacional de Ubajara, Trilha Araticum, Rio das Minas; maximumElevationInMeters: 524; verbatimCoordinates: 350'3"S, 4054'18"W; samplingProtocol: Manual; verbatimEventDate: 18.ii.13; individualCount: 2; sex: female; lifeStage: adult; recordedBy: Santos, A.P.M. | Takiya, D.M.; identifiedBy: Bruno Clarkson; institutionCode: DZRJ; basisOfRecord: PreservedSpecimen

b. country: Brazil; stateProvince: Ceará; municipality: Ubajara; locality: Parque Nacional de Ubajara, Trilha Araticum, Rio das Minas; maximumElevationInMeters: 524; verbatimCoordinates: 350'3"S, 4054'18"W; sampling Protocol: Manual; verbatimEventDate: 18.ii.13; individualCount: 13; sex: male; lifeStage: adult; recordedBy: Santos, A.P.M. | Takiya, D.M.; identifiedBy: Bruno Clarkson; institutionCode: DZRJ; basisOfRecord: PreservedSpecimen

c. country: Brazil; stateProvince: Ceará; municipality: Ubajara; locality: Parque Nacional de Ubajara, Trilha Araticum, Rio das Minas; maximumElevationInMeters: 524; verbatimCoordinates: 350'3"S, 4054'18"W; samplingProtocol: Manual; verbatimEventDate: 18.ii.13; individualCount: 15; sex: female; lifeStage: adult; recordedBy: Santos, A.P.M. | Takiya, D.M.; identifiedBy: Bruno Clarkson; institutionCode: DZRJ; basisOfRecord: PreservedSpecimen

d. country: Brazil; stateProvince: Ceará; municipality: Ubajara; locality: Parque Nacional de Ubajara, Trilha Araticum, Rio das Minas; maximumElevationInMeters: 524; verbatimCoordinates: 350'3"S, 4054'18"W; sampling Protocol: Manual; verbatimEventDate: 18.ii.13; individualCount: 3; lifeStage: immature; recordedBy: Santos, A.P.M. | Takiya, D.M.; identifiedBy: Bruno Clarkson; institutionCode: DZRJ; basisOfRecord: PreservedSpecimen 
Distribution: Brazil: PI!, CE!, AL. Argentina?

Notes: New species record for CE.

\section{Genus Paracymus Thomson, 1867}

\section{Paracymus rufocinctus Bruch, 1915}

\section{Material}

a. country: Brazil; stateProvince: Ceará; municipality: Ubajara; locality: Parque Nacional de Ubajara, Rio Cafundó, pouco acima da cachoeira; maximumElevationInMeters: 795; verbatimCoordinates: $3^{\circ} 50^{\prime} 13^{\prime \prime} \mathrm{S}, 40^{\circ} 54^{\prime} 35^{\prime \prime W}$; samplingProtocol: Malaise intercept trap; verbatimEventDate: 13.ii.13; individualCount: 1; sex: male; lifeStage: adult; recordedBy: Rafael, J.A. | Limeira-de-Oliveira, F. | Takiya, D.M. | Santos, A.P.M. | et al.; identifiedBy: Bruno Clarkson; institutionCode: DZRJ; basisOfRecord: PreservedSpecimen

Distribution: Widespread in South America. Brazil: PI, CE, PE. Argentina.

\section{Genus Phaenostoma Orchymont, 1937}

Notes: New genus record for CE.

\section{Phaenostoma sp. 1}

\section{Material}

a. country: Brazil; stateProvince: Ceará; municipality: Ubajara; locality: Parque Nacional de Ubajara, Ponte sobre Rio Miranda; maximumElevationInMeters: 792; verbatimCoordinates: $3^{\circ} 50^{\prime} 7.4^{\prime \prime} \mathrm{S}, 40^{\circ} 54^{\prime} 47.5^{\prime \prime} \mathrm{W}$; samplingProtocol: Pennsylvania light trap; verbatimEventDate: 15.ii.13; individualCount: 1; sex: male; lifeStage: adult; recordedBy: Santos, A.P.M. | Takiya, D.M.; identifiedBy: Bruno Clarkson; institutionCode: DZRJ; basisOfRecord: PreservedSpecimen

Notes: Undescribed species.

\section{Genus Phaenonotum Sharp, 1882}

\section{Phaenonotum sp. 1}

\section{Material}

a. country: Brazil; stateProvince: Ceará; municipality: Ubajara; locality: Parque Nacional de Ubajara, Rio das Minas, próximo ao Portão Araticum; maximumElevationInMeters: 328; verbatimCoordinates: 349'32.6"S, 4053'32.8"W; samplingProtocol: Malaise intercept trap; verbatimEventDate: 14.ii.13; individualCount: 1; sex: female; lifeStage: adult; recordedBy: Takiya, D.M. | Santos, A.P.M.; identifiedBy: Bruno Clarkson; institutionCode: DZRJ; basisOfRecord: PreservedSpecimen 


\section{Genus Tropisternus Solier, 1834}

\section{Tropisternus (Pristoternus) flavipalpis Sharp, 1883}

\section{Material}

a. country: Brazil; stateProvince: Ceará; municipality: Ubajara; locality: Parque Nacional de Ubajara, Ponte sobre Rio Miranda; maximumElevationInMeters: 792; verbatimCoordinates: $3^{\circ} 50^{\prime} 7.4^{\prime \prime} \mathrm{S}, 40^{\circ} 54^{\prime} 47.5^{\prime \prime} \mathrm{W}$; sampling Protocol: Pennsylvania light trap; verbatimEventDate: 15.ii.13; individualCount: 1; sex: female; lifeStage: adult; recordedBy: Santos, A.P.M. | Takiya, D.M.; identifiedBy: Bruno Clarkson; institutionCode: DZRJ; basisOfRecord: PreservedSpecimen

Distribution: Mexico. Brazil: CE!, RJ.

Notes: New species record for Northeastern Brazil.

\section{Family Torridincolidae}

Notes: New family record for CE.

\section{Genus Claudiella Reichardt \& Vanin, 1976}

Notes: New genus record for CE.

\section{Claudiella sp. 1}

\section{Materials}

a. country: Brazil; stateProvince: Ceará; municipality: Ubajara; locality: Parque Nacional de Ubajara, Trilha Araticum, Rio Cafundó; maximumElevationInMeters: 753; verbatimCoordinates: $3^{\circ} 50^{\prime} 12$ "S, 4054'31"W; samplingProtocol: Manual; verbatimEventDate: 17.ii.13; individualCount: 21; lifeStage: adult; recordedBy: Santos, A.P.M. | Takiya, D.M.; identifiedBy: Brunno H. L. Sampaio; institutionCode: DZRJ; basisOfRecord: PreservedSpecimen

b. $\quad$ country: Brazil; stateProvince: Ceará; municipality: Ubajara; locality: Parque Nacional de Ubajara, Trilha Araticum, Rio das Minas; maximumElevationInMeters: 524; verbatimCoordinates: $3^{\circ} 50$ '3"S, 4054'18"W; samplingProtocol: Manual; verbatimEventDate: 18.ii.13; individualCount: 69; lifeStage: adult; recordedBy: Santos, A.P.M. | Takiya, D.M.; identifiedBy: Brunno H. L. Sampaio; institutionCode: DZRJ; basisOfRecord: PreservedSpecimen

c. country: Brazil; stateProvince: Ceará; municipality: Ubajara; locality: Parque Nacional de Ubajara, Trilha Araticum, Rio das Minas; maximumElevationInMeters: 524; verbatimCoordinates: $3^{\circ} 50^{\prime} 3$ "S, 4054'18"W; sampling Protocol: Manual; verbatimEventDate: 22.iv.12; individualCount: 1; lifeStage: adult; recordedBy: Takiya, D.M.; identifiedBy: Brunno H. L. Sampaio; institutionCode: DZRJ; basisOfRecord: PreservedSpecimen

d. country: Brazil; stateProvince: Ceará; municipality: Ubajara; locality: Parque Nacional de Ubajara, Trilha Araticum, Rio das Minas; maximumElevationInMeters: 524; 
verbatimCoordinates: $3^{\circ} 50^{\prime} 3$ "S, $40^{\circ} 54^{\prime} 18^{\prime \prime} \mathrm{W}$; samplingProtocol: Manual; verbatimEventDate: 23.iv.12; individualCount: 5; lifeStage: adult; recordedBy: Takiya, D.M.; identifiedBy: Brunno H. L. Sampaio; institutionCode: DZRJ; basisOfRecord: PreservedSpecimen

e. country: Brazil; stateProvince: Ceará; municipality: Ubajara; locality: Parque Nacional de Ubajara, Rio Cafundó, pouco acima da cachoeira; maximumElevationInMeters: 795; verbatimCoordinates: $3^{\circ} 50^{\prime} 13^{\prime \prime S}, 40^{\circ} 54^{\prime} 35 " \mathrm{~W}$; samplingProtocol: Manual; verbatimEventDate: 23.iv.12; individualCount: 3; lifeStage: immature; recordedBy: Takiya, D.M.; identifiedBy: Brunno H. L. Sampaio; institutionCode: DZRJ; basisOfRecord: PreservedSpecimen

f. country: Brazil; stateProvince: Ceará; municipality: Ubajara; locality: Parque Nacional de Ubajara, Rio Cafundó, pouco acima da cachoeira; maximumElevationInMeters: 795; verbatimCoordinates: $3^{\circ} 50^{\prime} 13^{\prime \prime} \mathrm{S}, 40^{\circ} 54^{\prime} 35^{\prime \prime} \mathrm{W}$; samplingProtocol: Manual; verbatimEventDate: 23.iv.12; individualCount: 19; lifeStage: adult; recordedBy: Takiya, D.M.; identifiedBy: Brunno H. L. Sampaio; institutionCode: DZRJ; basisOfRecord: PreservedSpecimen

Notes: Undescribed species. See Fig. 19.

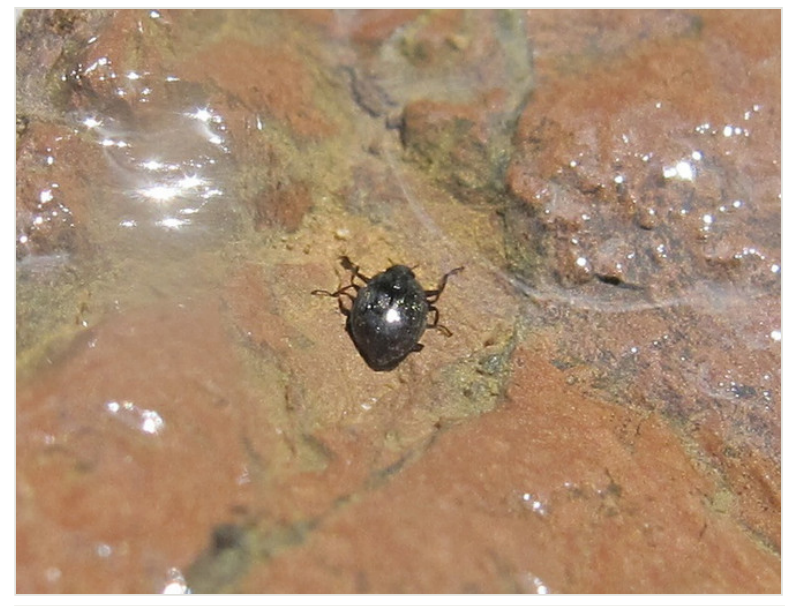

Figure 19.

Specimen of Claudiella sp. 1 collected at PNU-02, an undescribed species representing the first record of Torridincolidae for Ceará.

\section{Genus Ytu Reichardt, 1973}

Notes: New genus record for CE.

\section{Ytu sp. 1}

\section{Material}

a. country: Brazil; stateProvince: Ceará; municipality: Ubajara; locality: Parque Nacional de Ubajara, Trilha Araticum, Rio das Minas; maximumElevationInMeters: 524; 
verbatimCoordinates: $3^{\circ} 50^{\prime} 3^{\prime \prime S}, 40^{\circ} 54^{\prime} 18 " \mathrm{~W}$; samplingProtocol: Manual; verbatimEventDate: 18.ii.13; individualCount: 64; lifeStage: adult; recordedBy: Santos, A.P.M. | Takiya, D.M.; identifiedBy: Brunno H. L. Sampaio; institutionCode: DZRJ; basisOfRecord: PreservedSpecimen

Notes: Undescribed species.

\section{Order Diptera}

\section{Family Empididae}

\section{Subfamily Hemerodromiinae}

Notes: Subfamily firstly recorded from CE in Câmara et al. 2015.

\section{Genus Hemerodromia Meigen, 1823}

Notes: Genus firstly recorded from CE in Câmara et al. 2015.

\section{Hemerodromia brevicercata Câmara, Takiya, Plant \& Rafael, 2015}

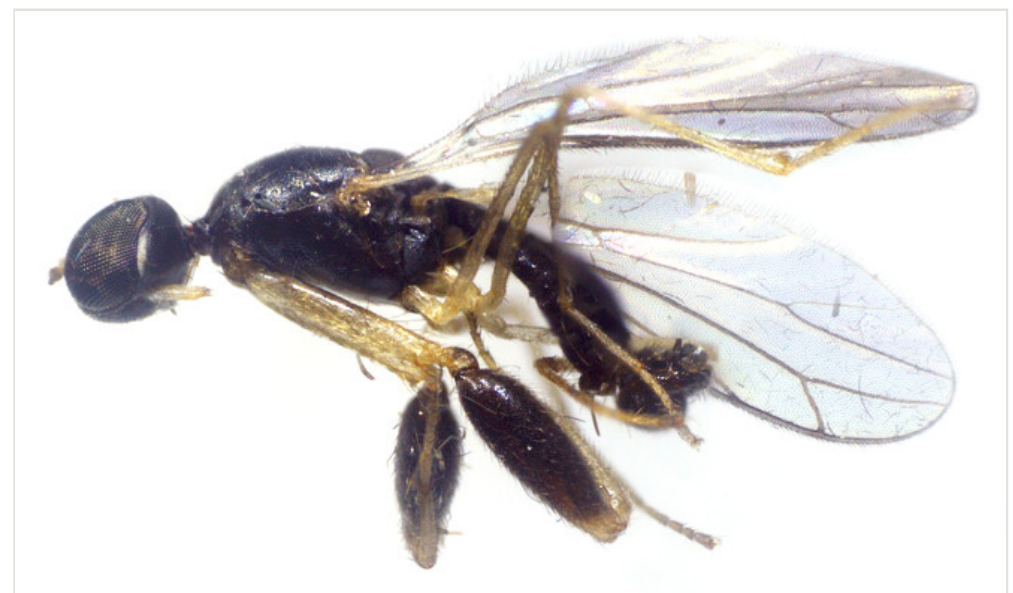

Figure 20.

Lateral habitus of male holotype of Hemerodromia brevicercata collected at PNU-07 (CZMA), species described based on material collected during this project.

\section{Material}

a. country: Brazil; stateProvince: Ceará; municipality: Ubajara; locality: Parque Nacional de Ubajara, Rio Cafundó, pouco acima da cachoeira; maximumElevationInMeters: 795; verbatimCoordinates: $3^{\circ} 50^{\prime} 13^{\prime \prime S}, 40^{\circ} 54^{\prime} 35 " \mathrm{~W}$; samplingProtocol: Malaise intercept trap; verbatimEventDate: 13.ii.13; individualCount: 258; lifeStage: adult; recordedBy: Rafael, 
J.A. | Limeira-de-Oliveira, F. | Takiya, D.M. | Santos, A.P.M. | et al.; identifiedBy: Josenir Teixeira Câmara; institutionCode: DZRJ; basisOfRecord: PreservedSpecimen

Distribution: Brazil: CE.

Notes: Species described in Câmara et al. 2015. See Fig. 20.

\section{Hemerodromia membranosa Câmara, Takiya, Plant \& Rafael, 2015}

\section{Material}

a. country: Brazil; stateProvince: Ceará; municipality: Ubajara; locality: Parque Nacional de Ubajara, Rio Cafundó, pouco acima da cachoeira; maximumElevationInMeters: 795; verbatimCoordinates: $3^{\circ} 50^{\prime} 13^{\prime \prime} \mathrm{S}, 40^{\circ} 54^{\prime} 35 \mathrm{~W} \mathrm{~W}$; samplingProtocol: Malaise intercept trap; verbatimEventDate: 14.ii.13; individualCount: 33; lifeStage: adult; recordedBy: Rafael, J.A. | Limeira-de-Oliveira, F. | Takiya, D.M. | Santos, A.P.M. | et al.; identifiedBy: Josenir Teixeira Câmara; institutionCode: DZRJ; basisOfRecord: PreservedSpecimen

Distribution: Brazil: CE.

Notes: Species described in Câmara et al. 2015.

\section{Hemerodromia mesomelaena* Bezzi, 1909}

\section{Material}

a. country: Brazil; stateProvince: Ceará; municipality: Ubajara; locality: Parque Nacional de Ubajara, Rio Cafundó, pouco acima da cachoeira; maximumElevationInMeters: 795; verbatimCoordinates: $3^{\circ} 50^{\prime} 13^{\prime \prime S}, 40^{\circ} 54^{\prime} 35$ "W; samplingProtocol: Malaise intercept trap; verbatimEventDate: 14.ii.13; individualCount: 15; lifeStage: adult; recordedBy: Rafael, J.A. | Limeira-de-Oliveira, F. | Takiya, D.M. | Santos, A.P.M. | et al.; identifiedBy: Josenir Teixeira Câmara; institutionCode: DZRJ; basisOfRecord: PreservedSpecimen

Distribution: Brazil: PI!, CE!, PR. Peru. Argentina.

Notes: New species record for Northeastern Brazil.

\section{Hemerodromia mourai Câmara, Takiya, Plant \& Rafael, 2015}

\section{Material}

a. $\quad$ country: Brazil; stateProvince: Ceará; municipality: Ubajara; locality: Parque Nacional de Ubajara, Rio Cafundó, pouco acima da cachoeira; maximumElevationInMeters: 795; verbatimCoordinates: $3^{\circ} 50^{\prime} 13^{\prime \prime S}, 40^{\circ} 54^{\prime} 35 " \mathrm{~W}$; samplingProtocol: Malaise intercept trap; verbatimEventDate: 14.ii.13; individualCount: 18; lifeStage: adult; recordedBy: Rafael, J.A. | Limeira-de-Oliveira, F. | Takiya, D.M. | Santos, A.P.M. | et al.; identifiedBy: Josenir Teixeira Câmara; institutionCode: DZRJ; basisOfRecord: PreservedSpecimen

Distribution: Brazil: CE.

Notes: Species described in Câmara et al. 2015. See Fig. 21. 


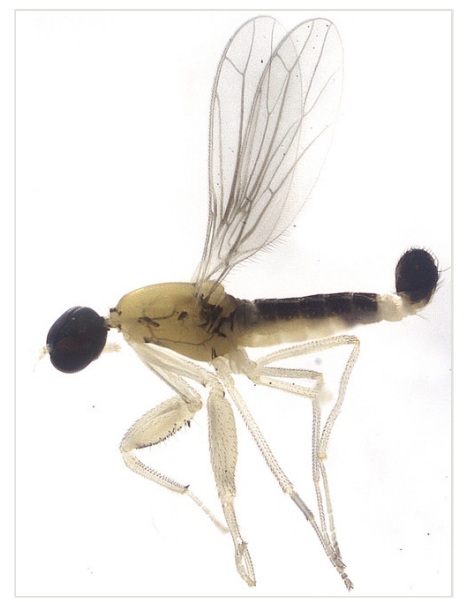

Figure 21.

Lateral habitus of male holotype of Hemerodromia mourai collected at PNU-07 (CZMA), species described based on material collected during this project.

\section{Hemerodromia ubajaraensis Câmara, Takiya, Plant \& Rafael, 2015}

\section{Material}

a. country: Brazil; stateProvince: Ceará; municipality: Ubajara; locality: Parque Nacional de Ubajara, Rio Cafundó, pouco acima da cachoeira; maximumElevationInMeters: 795; verbatimCoordinates: $3^{\circ} 50^{\prime} 13^{\prime \prime}$ S, $40^{\circ} 54^{\prime} 35 " \mathrm{~W}$; samplingProtocol: Malaise intercept trap; verbatimEventDate: 18.ii.13; individualCount: 5; lifeStage: adult; recordedBy: Rafael, J.A. I Limeira-de-Oliveira, F. | Takiya, D.M. | Santos, A.P.M. | et al.; identifiedBy: Josenir Teixeira Câmara; institutionCode: DZRJ; basisOfRecord: PreservedSpecimen

Distribution: Brazil: CE.

Notes: Species described in Câmara et al. 2015. See Fig. 22.

\section{Hemerodromia sp. 1}

\section{Material}

a. country: Brazil; stateProvince: Ceará; municipality: Ubajara; locality: Parque Nacional de Ubajara, Rio Cafundó, pouco acima da cachoeira; maximumElevationInMeters: 795; verbatimCoordinates: $3^{\circ} 50^{\prime} 13^{\prime \prime} \mathrm{S}, 40^{\circ} 54^{\prime} 35^{\prime \prime} \mathrm{W}$; samplingProtocol: Malaise intercept trap; verbatimEventDate: 14.ii.13; individualCount: 3; lifeStage: adult; recordedBy: Rafael, J.A. | Limeira-de-Oliveira, F. | Takiya, D.M. | Santos, A.P.M. | et al.; identifiedBy: Josenir Teixeira Câmara; institutionCode: DZRJ; basisOfRecord: PreservedSpecimen

Notes: Undescribed species. 


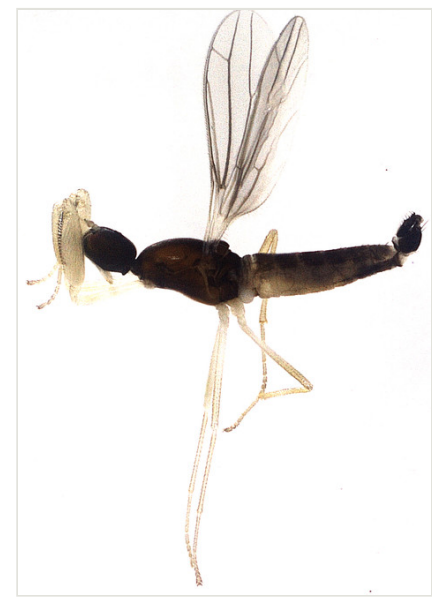

Figure 22.

Lateral habitus of male holotype of Hemerodromia ubajaraensis collected at PNU-07 (CZMA), species described based on material collected during this project.

\section{Genus Metachela Coquillett, 1903}

Notes: New genus record for CE.

\section{Metachela sp. 1}

\section{Material}

a. country: Brazil; stateProvince: Ceará; municipality: Ubajara; locality: Parque Nacional de Ubajara, Rio Cafundó, pouco acima da cachoeira; maximumElevationInMeters: 795; verbatimCoordinates: $3^{\circ} 50^{\prime} 13^{\prime \prime} \mathrm{S}, 40^{\circ} 54^{\prime} 35 \mathrm{WW}$; samplingProtocol: Malaise intercept trap; verbatimEventDate: 24.iv.12; individualCount: 28; lifeStage: adult; recordedBy: Rafael, J.A. | Limeira-de-Oliveira, F. | Takiya, D.M. | et al.; identifiedBy: Josenir Teixeira Câmara; institutionCode: DZRJ; basisOfRecord: PreservedSpecimen

Notes: Undescribed species.

\section{Metachela sp. 2}

Material

a. country: Brazil; stateProvince: Ceará; municipality: Ubajara; locality: Parque Nacional de Ubajara, Rio Cafundó, pouco acima da cachoeira; maximumElevationInMeters: 795; verbatimCoordinates: $3^{\circ} 50^{\prime} 13^{\prime \prime} \mathrm{S}, 40^{\circ} 54^{\prime} 35^{\prime \prime} \mathrm{W}$; samplingProtocol: Malaise intercept trap; verbatimEventDate: 14.ii.13; individualCount: 15; lifeStage: adult; recordedBy: Rafael, J.A. | Limeira-de-Oliveira, F. | Takiya, D.M. | Santos, A.P.M. | et al.; identifiedBy: Josenir Teixeira Câmara; institutionCode: DZRJ; basisOfRecord: PreservedSpecimen

Notes: Undescribed species. 


\section{Genus Neoplasta Coquillett, 1895}

Notes: New genus record for CE.

\section{Neoplasta sp. 1}

\section{Material}

a. country: Brazil; stateProvince: Ceará; municipality: Ubajara; locality: Parque Nacional de Ubajara, Rio Cafundó, pouco acima da cachoeira; maximumElevationInMeters: 795; verbatimCoordinates: $3^{\circ} 50^{\prime} 13^{\prime \prime S}, 40^{\circ} 54^{\prime} 35 \mathrm{WW}$; samplingProtocol: Malaise intercept trap; verbatimEventDate: 18.ii.13; individualCount: 1986; lifeStage: adult; recordedBy: Rafael, J.A. | Limeira-de-Oliveira, F. | Takiya, D.M. | Santos, A.P.M. | et al.; identifiedBy: Josenir Teixeira Câmara; institutionCode: DZRJ; basisOfRecord: PreservedSpecimen

Notes: Undescribed species.

\section{Neoplasta sp. 2}

\section{Material}

a. country: Brazil; stateProvince: Ceará; municipality: Ubajara; locality: Parque Nacional de Ubajara, Rio Cafundó, pouco acima da cachoeira; maximumElevationInMeters: 795; verbatimCoordinates: $3^{\circ} 50^{\prime} 13^{\prime \prime} \mathrm{S}, 40^{\circ} 54^{\prime} 35 \mathrm{WW}$; samplingProtocol: Malaise intercept trap; verbatimEventDate: 24.iv.12; individualCount: 326; lifeStage: adult; recordedBy: Rafael, J.A. | Limeira-de-Oliveira, F. | Takiya, D.M. | et al.; identifiedBy: Josenir Teixeira Câmara; institutionCode: DZRJ; basisOfRecord: PreservedSpecimen

Notes: Undescribed species.

\section{Order Ephemeroptera}

\section{Family Leptophlebiidae}

Notes: New family record for CE.

\section{Genus Farrodes Peters, 1971}

Notes: New genus record for CE.

\section{Farrodes tepui* Domínguez, Molineri \& Peters, 1996}

\section{Material}

a. country: Brazil; stateProvince: Ceará; municipality: Ubajara; locality: Parque Nacional de Ubajara, Trilha Araticum, Rio das Minas na altura da trilha do teleférico; maximumElevationInMeters: 420; verbatimCoordinates: $3^{\circ} 49^{\prime} 58^{\prime \prime S}, 40^{\circ} 53^{\prime} 53^{\prime \prime} \mathrm{W}$; sampling Protocol: Pennsylvania light trap; verbatimEventDate: 14.ii.13; individualCount: 
1; sex: male; lifeStage: immature; recordedBy: Santos, A.P.M. | Takiya, D.M.; identifiedBy: Inês Corrêa Gonçalves; institutionCode: DZRJ; basisOfRecord: PreservedSpecimen

Distribution: Venezuela. Brazil: PI!, CE!, PE.

Notes: New species record for CE.

\section{Order Hemiptera}

\section{Infraorder Gerromorpha}

\section{Family Gerridae}

\section{Genus Limnogonus Stål, 1868}

Notes: Genus firstly recorded from CE in Cordeiro and Moreira 2015.

\section{Limnogonus profugus Drake \& Harris, 1930}

\section{Material}

a. $\quad$ country: Brazil; stateProvince: Ceará; municipality: Ubajara; locality: Parque Nacional de Ubajara, Trilha Araticum, Rio das Minas; maximumElevationInMeters: 524; verbatimCoordinates: $3^{\circ} 50^{\prime} 3$ "S, $40^{\circ} 54^{\prime} 18^{\prime \prime} \mathrm{W}$; samplingProtocol: Manual; verbatimEventDate: 14.ii.13; individualCount: 1; sex: female; lifeStage: adult; recordedBy: Santos, A.P.M. | Takiya, D.M.; identifiedBy: Felipe F. F. Moreira; institutionCode: DZRJ; basisOfRecord: PreservedSpecimen

Distribution: Brazil: CE, PB, PE, MT, GO, MG, MS, SP, RJ. Peru. Paraguay. Argentina. Notes: Species firstly recorded from CE in Cordeiro and Moreira 2015.

\section{Family Veliidae}

\section{Genus Rhagovelia Mayr, 1865}

Notes: Genus firstly recorded from CE in Cordeiro and Moreira 2015.

\section{Rhagovelia whitei (Breddin, 1898)}

\section{Materials}

a. country: Brazil; stateProvince: Ceará; municipality: Ubajara; locality: Parque Nacional de Ubajara, Trilha Araticum, Rio das Minas; maximumElevationInMeters: 524; verbatimCoordinates: $3^{\circ} 50^{\prime} 3$ "S, $40^{\circ} 54^{\prime} 18$ "W; samplingProtocol: Manual; verbatimEventDate: 23.iv.12; individualCount: 1; sex: male; lifeStage: adult; recordedBy: 
Takiya, D.M.; identifiedBy: Felipe F. F. Moreira; institutionCode: DZRJ; basisOfRecord: PreservedSpecimen

b. $\quad$ country: Brazil; stateProvince: Ceará; municipality: Ubajara; locality: Parque Nacional de Ubajara, Trilha Araticum, Rio das Minas; maximumElevationInMeters: 524; verbatimCoordinates: $3^{\circ} 50$ '3"S, 4054'18"W; samplingProtocol: Manual; verbatimEventDate: 23.iv.12; individualCount: 1; sex: female; lifeStage: adult; recordedBy: Takiya, D.M.; identifiedBy: Felipe F. F. Moreira; institutionCode: DZRJ; basisOfRecord: PreservedSpecimen

Distribution: Brazil: PA, CE, MA, MT, GO, MG, MS, SP. Paraguay.

Notes: Species firstly recorded from CE in Cordeiro and Moreira 2015.

\section{Rhagovelia sp. 1}

\section{Materials}

a. country: Brazil; stateProvince: Ceará; municipality: Ubajara; locality: Parque Nacional de Ubajara, Trilha Araticum, Rio das Minas; maximumElevationInMeters: 524; verbatimCoordinates: $3^{\circ} 50^{\prime} 3$ "S, 4054'18"W; samplingProtocol: Manual; verbatimEventDate: 17.ii.13; individualCount: 12; lifeStage: immature; recordedBy: Takiya, D.M.; identifiedBy: Felipe F. F. Moreira; institutionCode: DZRJ; basisOfRecord: PreservedSpecimen

b. country: Brazil; stateProvince: Ceará; municipality: Ubajara; locality: Parque Nacional de Ubajara, Trilha Samambaia, Rio Gameleira; maximumElevationInMeters: 874; verbatimCoordinates: $3^{\circ} 50^{\prime 25}$ "S, 4054'19"W; samplingProtocol: Manual; verbatimEventDate: 24.iv.12; individualCount: 1; sex: female; lifeStage: adult; recordedBy: Takiya, D.M.; identifiedBy: Felipe F. F. Moreira; institutionCode: DZRJ; basisOfRecord: PreservedSpecimen

\section{Infraorder Nepomorpha}

\section{Family Belostomatidae}

\section{Genus Belostoma Latreille, 1807}

\section{Belostoma sp. 1*}

\section{Material}

a. country: Brazil; stateProvince: Ceará; municipality: Ubajara; locality: Parque Nacional de Ubajara, Trilha Samambaia, Rio Gameleira; maximumElevationInMeters: 874; verbatimCoordinates: $3^{\circ} 50 ' 25$ "S, 4054'19"W; samplingProtocol: Manual; verbatimEventDate: 21.iv.12; individualCount: 4; lifeStage: immature; recordedBy: Takiya, D.M.; identifiedBy: Julianna Freires Barbosa; institutionCode: DZRJ; basisOfRecord: PreservedSpecimen 


\section{Family Corixidae}

\section{Genus Tenagobia Bergroth, 1899}

\section{Tenagobia sp. 1}

\section{Materials}

a. country: Brazil; stateProvince: Ceará; municipality: Ubajara; locality: Parque Nacional de Ubajara, Trilha Samambaia, Rio Gameleira; maximumElevationInMeters: 874; verbatimCoordinates: $3^{\circ} 50^{\prime} 25 " \mathrm{~S}, 40^{\circ} 54^{\prime} 19^{\prime \prime} \mathrm{W}$; samplingProtocol: Pennsylvania light trap; verbatimEventDate: 21.iv.12; individualCount: 2; sex: male; lifeStage: adult; recordedBy: Takiya, D.M. | Câmara, J.T.; identifiedBy: Julianna Freires Barbosa; institutionCode: DZRJ; basisOfRecord: PreservedSpecimen

b. $\quad$ country: Brazil; stateProvince: Ceará; municipality: Ubajara; locality: Parque Nacional de Ubajara, Trilha Samambaia, Rio Gameleira; maximumElevationInMeters: 874; verbatimCoordinates: $3^{\circ} 50^{\prime} 25^{\prime \prime}$ S, $40^{\circ} 54^{\prime} 19^{\prime \prime}$; samplingProtocol: Pennsylvania light trap; verbatimEventDate: 21.iv.12; individualCount: 7; sex: female; lifeStage: adult; recordedBy: Takiya, D.M. | Câmara, J.T.; identifiedBy: Julianna Freires Barbosa; institutionCode: DZRJ; basisOfRecord: PreservedSpecimen

\section{Family Gelastocoridae}

Notes: New family record for CE.

\section{Genus Gelastocoris Kirkaldy, 1897}

Notes: New genus record for CE.

\section{Gelastocoris angulatus (Melin, 1929)}

\section{Materials}

a. country: Brazil; stateProvince: Ceará; municipality: Ubajara; locality: Parque Nacional de Ubajara, Cachoeira do Cafundó; maximumElevationInMeters: 783; verbatimCoordinates: 350'12"S, 4054'35"W; samplingProtocol: Manual; verbatimEventDate: 16.ii.13; individualCount: 1; sex: male; lifeStage: adult; recordedBy: Santos, A.P.M. | Takiya, D.M.; identifiedBy: Julianna Freires Barbosa; institutionCode: DZRJ; basisOfRecord: PreservedSpecimen

b. $\quad$ country: Brazil; stateProvince: Ceará; municipality: Ubajara; locality: Parque Nacional de Ubajara, Trilha Araticum, Rio das Minas; maximumElevationInMeters: 524; verbatimCoordinates: $3^{\circ} 50$ '3"S, 4054'18"W; samplingProtocol: YPT; verbatimEventDate: 22.iv.12; individualCount: 1; sex: male; lifeStage: adult; recordedBy: Câmara, J.T.; identifiedBy: Julianna Freires Barbosa; institutionCode: DZRJ; basisOfRecord: PreservedSpecimen

Distribution: Guyana. Suriname. Brazil: PA, AM, CE!, MT, BA, GO, MG, SP, RJ. Bolivia. Paraguay. Argentina. 
Notes: New species record for CE.

\section{Gelastocoris sp.}

\section{Materials}

a. country: Brazil; stateProvince: Ceará; municipality: Ubajara; locality: Parque Nacional de Ubajara, Trilha Araticum, Rio das Minas; maximumElevationInMeters: 524; verbatimCoordinates: $3^{\circ} 50^{\prime} 3$ "S, $40^{\circ} 54^{\prime} 18^{\prime \prime} \mathrm{W}$; samplingProtocol: Manual; verbatimEventDate: 22.iv.12; individualCount: 1; lifeStage: immature; recordedBy: Takiya, D.M.; identifiedBy: Julianna Freires Barbosa; institutionCode: DZRJ; basisOfRecord: PreservedSpecimen

b. country: Brazil; stateProvince: Ceará; municipality: Ubajara; locality: Parque Nacional de Ubajara, Trilha Araticum, Rio das Minas; maximumElevationInMeters: 524; verbatimCoordinates: $3^{\circ} 50^{\prime} 3 " \mathrm{~S}, 40^{\circ} 54^{\prime} 18 \mathrm{~W} \mathrm{~W}$; samplingProtocol: YPT; verbatimEventDate: 22.iv.12; individualCount: 1; sex: male; lifeStage: adult; recordedBy: Câmara, J.T.; identifiedBy: Julianna Freires Barbosa; institutionCode: DZRJ; basisOfRecord: PreservedSpecimen

\section{Family Naucoridae}

Notes: New family record for CE.

\section{Genus Limnocoris Stål, 1858}

Notes: New genus record for CE.

\section{Limnocoris sp. 1}

\section{Material}

a. country: Brazil; stateProvince: Ceará; municipality: Ubajara; locality: Parque Nacional de Ubajara, Trilha Araticum, Rio das Minas; maximumElevationInMeters: 524; verbatimCoordinates: $3^{\circ} 50^{\prime} 3$ "S, 4054'18"W; samplingProtocol: Manual; verbatimEventDate: 14.ii.13; individualCount: 3; sex: female; lifeStage: adult; recordedBy: Santos, A.P.M. | Takiya, D.M.; identifiedBy: Julianna Freires Barbosa; institutionCode: DZRJ; basisOfRecord: PreservedSpecimen

\section{Family Notonectidae}

\section{Genus Martarega White, 1879}

\section{Martarega bentoi* Truxal, 1949}

\section{Materials}

a. $\quad$ country: Brazil; stateProvince: Ceará; municipality: Ubajara; locality: Parque Nacional de Ubajara, Cachoeira do Cafundó; maximumElevationInMeters: 783; verbatimCoordinates: 
$3^{\circ} 50^{\prime} 12^{\prime \prime S}, 40^{\circ} 54^{\prime} 35 " \mathrm{~W}$; samplingProtocol: Manual; verbatimEventDate: 16.ii.13; individualCount: 9; sex: male; lifeStage: adult; recordedBy: Santos, A.P.M. | Takiya, D.M.; identifiedBy: Julianna Freires Barbosa; institutionCode: DZRJ; basisOfRecord: PreservedSpecimen

b. country: Brazil; stateProvince: Ceará; municipality: Ubajara; locality: Parque Nacional de Ubajara, Cachoeira do Cafundó; maximumElevationInMeters: 783; verbatimCoordinates: 350'12"S, 4054'35"W; samplingProtocol: Manual; verbatimEventDate: 16.ii.13; individualCount: 3; sex: female; lifeStage: adult; recordedBy: Santos, A.P.M. | Takiya, D.M.; identifiedBy: Julianna Freires Barbosa; institutionCode: DZRJ; basisOfRecord: PreservedSpecimen

c. country: Brazil; stateProvince: Ceará; municipality: Ubajara; locality: Parque Nacional de Ubajara, Cachoeira do Cafundó; maximumElevationInMeters: 783; verbatimCoordinates: 350'12"S, 4054'35"W; samplingProtocol: Manual; verbatimEventDate: 16.ii.13; individualCount: 28; lifeStage: immature; recordedBy: Santos, A.P.M. | Takiya, D.M.; identifiedBy: Julianna Freires Barbosa; institutionCode: DZRJ; basisOfRecord: PreservedSpecimen

Distribution: Brazil: PI, CE!, PE, MT, MG, RJ. Argentina.

Notes: New species record for CE.

\section{Order Odonata}

\section{Suborder Anisoptera}

\section{Family Aeshnidae}

\section{Genus Castoraeschna Calvert, 1952}

\section{Castoraeschna corbeti Carvalho, Pinto \& Ferreira-Jr, 2009}

\section{Materials}

a. country: Brazil; stateProvince: Ceará; municipality: Ubajara; locality: Parque Nacional de Ubajara, Rio das Minas, próximo ao Portão Araticum; maximumElevationlnMeters: 328; verbatimCoordinates: $3^{\circ} 49^{\prime} 32.6$ "S, 40 $53^{\circ} 32.8^{\prime \prime W}$; samplingProtocol: Malaise intercept trap; verbatimEventDate: 16.ii.13; individualCount: 1; sex: male; lifeStage: adult; recordedBy: Rafael, J.A. | Limeira-de-Oliveira, F. | Takiya, D.M. | Santos, A.P.M. | et al.; identifiedBy: Alcimar do Lago Carvalho | Ângelo Parise Pinto; institutionCode: DZRJ; basisOfRecord: PreservedSpecimen

b. country: Brazil; stateProvince: Ceará; municipality: Ubajara; locality: Parque Nacional de Ubajara, Trilha Araticum, Rio das Minas; maximumElevationInMeters: 524; verbatimCoordinates: $3^{\circ} 50^{\prime} 3$ "S, 4054'18"W; sampling Protocol: Manual; verbatimEventDate: 18.ii.13; individualCount: 1; sex: male; lifeStage: adult; recordedBy: Santos, A.P.M. | Takiya, D.M.; identifiedBy: Alcimar do Lago Carvalho | Ângelo Parise Pinto; institutionCode: DZRJ; basisOfRecord: PreservedSpecimen

Distribution: Brazil: PA, CE!. 
Notes: New species record for CE.

\section{Genus Gynacantha Rambur, 1842}

\section{Gynacantha nervosa* Rambur, 1842}

\section{Material}

a. country: Brazil; stateProvince: Ceará; municipality: Ubajara; locality: Parque Nacional de Ubajara, Portão Neblina; maximumElevationInMeters: 849; verbatimCoordinates: $3^{\circ}$ 50'18"S, 4053'54"W; samplingProtocol: Manual; verbatimEventDate: 17.ii.13; individualCount: 1; sex: female; lifeStage: adult; recordedBy: Santos, A.P.M. | Takiya, D.M.; identifiedBy: Ângelo Parise Pinto; institutionCode: DZRJ; basisOfRecord: PreservedSpecimen

Distribution: USA south to Panama. Trinidad and Tobago. Colombia. Venezuela. Guyana. Suriname. French Guiana. Brazil: RR, PI!, CE, PE, MT, MG, MS, ES, RJ. Ecuador. Peru. Bolivia.

\section{Family Gomphidae}

\section{Genus Progomphus Selys, 1854}

\section{Progomphus complicatus Selys, 1854}

\section{Materials}

a. country: Brazil; stateProvince: Ceará; municipality: Ubajara; locality: Parque Nacional de Ubajara, Trilha Araticum, Rio das Minas na altura da trilha do teleférico; maximumElevationInMeters: 420; verbatimCoordinates: 349'58"S, 4053'53"W; samplingProtocol: Malaise intercept trap; verbatimEventDate: 14.ii.13; individualCount: 1; sex: male; lifeStage: adult; recordedBy: Rafael, J.A. | Limeira-de-Oliveira, F. | Takiya, D.M. | Santos, A.P.M. | et al.; identifiedBy: Ângelo Parise Pinto | Marcus Vinicius O. de Almeida; institutionCode: CZMA; basisOfRecord: PreservedSpecimen

b. $\quad$ country: Brazil; stateProvince: Ceará; municipality: Ubajara; locality: Parque Nacional de Ubajara, Trilha Araticum, Rio das Minas na altura da trilha do teleférico; maximumElevationInMeters: 420 ; verbatimCoordinates: $3^{\circ} 49^{\prime} 58^{\prime \prime} \mathrm{S}, 40^{\circ} 53^{\prime} 53^{\prime \prime} \mathrm{W}$; samplingProtocol: Malaise intercept trap; verbatimEventDate: 14.ii.13; individualCount: 1; sex: male; lifeStage: adult; recordedBy: Rafael, J.A. | Limeira-de-Oliveira, F. | Takiya, D.M. | Santos, A.P.M. | et al.; identifiedBy: Ângelo Parise Pinto | Marcus Vinicius O. de Almeida; institutionCode: DZRJ; basisOfRecord: PreservedSpecimen

c. country: Brazil; stateProvince: Ceará; municipality: Ubajara; locality: Parque Nacional de Ubajara, Rio das Minas, próximo ao Portão Araticum; maximumElevationInMeters: 328; verbatimCoordinates: $3^{\circ} 49^{\prime} 32.6^{\prime \prime S}, 40^{\circ} 53^{\prime} 32.8^{\prime \prime} \mathrm{W}$; samplingProtocol: Malaise intercept trap; verbatimEventDate: 14.ii.13; individualCount: 1; sex: female; lifeStage: adult; recordedBy: Rafael, J.A. | Limeira-de-Oliveira, F. | Takiya, D.M. | Santos, A.P.M. | et al.; identifiedBy: Ângelo Parise Pinto | Marcus Vinicius O. de Almeida; institutionCode: DZRJ; basisOfRecord: PreservedSpecimen 
Distribution: Brazil: CE, BA, MG, ES, SP, RJ, SC, RS. Paraguay. Argentina.

Notes: Species firstly recorded from CE in De Almeida et al. 2013.

\section{Progomphus dorsopallidus Byers, 1934}

\section{Materials}

a. country: Brazil; stateProvince: Ceará; municipality: Ubajara; locality: Parque Nacional de Ubajara, Trilha Araticum, Rio das Minas na altura da trilha do teleférico; maximumElevationInMeters: 420 ; verbatimCoordinates: $3^{\circ} 49^{\prime} 58^{\prime \prime S}, 40^{\circ} 53^{\prime} 53^{\prime \prime} \mathrm{W}$; samplingProtocol: Malaise intercept trap; verbatimEventDate: 14.ii.13; individualCount: 1; sex: female; lifeStage: adult; recordedBy: Rafael, J.A. | Limeira-de-Oliveira, F. | Takiya, D.M. | Santos, A.P.M. | et al.; identifiedBy: Ângelo Parise Pinto | Marcus Vinicius O. de Almeida; institutionCode: CZMA; basisOfRecord: PreservedSpecimen

b. $\quad$ country: Brazil; stateProvince: Ceará; municipality: Ubajara; locality: Parque Nacional de Ubajara, Trilha Araticum, Rio das Minas na altura da trilha do teleférico; maximumElevationInMeters: 420; verbatimCoordinates: $3^{\circ} 49^{\prime} 58^{\prime \prime} \mathrm{S}, 40^{\circ} 53^{\prime} 53^{\prime \prime} \mathrm{W}$; samplingProtocol: Malaise intercept trap; verbatimEventDate: 14.ii.13; individualCount: 1; sex: female; lifeStage: adult; recordedBy: Rafael, J.A. | Limeira-de-Oliveira, F. | Takiya, D.M. | Santos, A.P.M. | et al.; identifiedBy: Ângelo Parise Pinto | Marcus Vinicius O. de Almeida; institutionCode: DZRJ; basisOfRecord: PreservedSpecimen

c. country: Brazil; stateProvince: Ceará; municipality: Ubajara; locality: Parque Nacional de Ubajara, Rio das Minas, próximo ao Portão Araticum; maximumElevationlnMeters: 328; verbatimCoordinates: $3^{\circ} 49^{\prime} 32.6^{\prime \prime} \mathrm{S}, 40^{\circ} 53^{\prime} 32.8^{\prime \prime} \mathrm{W}$; samplingProtocol: Malaise intercept trap; verbatimEventDate: 14.ii.13; individualCount: 1; sex: female; lifeStage: adult; recordedBy: Rafael, J.A. | Limeira-de-Oliveira, F. | Takiya, D.M. | Santos, A.P.M. | et al.; identifiedBy: Ângelo Parise Pinto | Marcus Vinicius O. de Almeida; institutionCode: DZRJ; basisOfRecord: PreservedSpecimen

Distribution: Trinidad and Tobago. Venezuela. Guyana. Brazil: CE, ES.

\section{Family Libellulidae}

\section{Genus Brechmorhoga Kirby, 1889}

\section{Brechmorhoga nubecula (Rambur, 1842)}

\section{Material}

a. country: Brazil; stateProvince: Ceará; municipality: Ubajara; locality: Parque Nacional de Ubajara, Trilha Araticum, Rio das Minas; maximumElevationInMeters: 524; verbatimCoordinates: $3^{\circ} 50^{\prime} 3$ "S, $40^{\circ} 54^{\prime} 18^{\prime \prime} \mathrm{W}$; samplingProtocol: Manual; verbatimEventDate: 18.ii.13; individualCount: 1; sex: male; lifeStage: adult; recordedBy: Santos, A.P.M. | Takiya, D.M.; identifiedBy: Ângelo Parise Pinto; institutionCode: DZRJ; basisOfRecord: PreservedSpecimen 
Distribution: Mexico. Belize. Costa Rica. Panama. Trinidad and Tobago. Colombia. Venezuela. Brazil: CE!, MT, BA, MG, ES, SP, RJ, SC. Ecuador. Peru. Paraguay. Argentina

Notes: New species record for CE.

\section{Genus Dasythemis Karsch, 1889}

\section{Dasythemis esmeralda Ris, 1910}

\section{Material}

a. country: Brazil; stateProvince: Ceará; municipality: Ubajara; locality: Parque Nacional de Ubajara, Trilha Samambaia, Rio Gameleira; maximumElevationInMeters: 874; verbatimCoordinates: $3^{\circ} 50^{\prime} 25^{\prime \prime}$ S, 4054'19"W; samplingProtocol: Manual; verbatimEventDate: 24.iv.12; individualCount: 1; sex: male; lifeStage: adult; recordedBy: Takiya, D.M. | Cavichioli, R.R.; identifiedBy: Alcimar do Lago Carvalho | Ângelo Parise Pinto; institutionCode: DZRJ; basisOfRecord: PreservedSpecimen

Distribution: Trinidad and Tobago. Colombia. Venezuela. Suriname. French Guiana. Brazil: AM, CE, PE, MT, RO, GO, MS, SP, RJ. Ecuador. Peru. Bolivia.

\section{Genus Dythemis Hagen, 1861}

\section{Dythemis nigra Martin, 1897}

\section{Materials}

a. country: Brazil; stateProvince: Ceará; municipality: Ubajara; locality: Parque Nacional de Ubajara, Rio das Minas, próximo ao Portão Araticum; maximumElevationInMeters: 328; verbatimCoordinates: $3^{\circ} 49^{\prime} 32.6^{\prime \prime S}, 40^{\circ} 53^{\prime} 32.8^{\prime \prime W}$; samplingProtocol: Malaise intercept trap; verbatimEventDate: 14.ii.13; individualCount: 1; sex: female; lifeStage: adult; recordedBy: Rafael, J.A. | Limeira-de-Oliveira, F. | Takiya, D.M. | Santos, A.P.M. | et al.; identifiedBy: Ângelo Parise Pinto | Marcus Vinicius O. de Almeida; institutionCode: CZMA; basisOfRecord: PreservedSpecimen

b. country: Brazil; stateProvince: Ceará; municipality: Ubajara; locality: Parque Nacional de Ubajara, Trilha Araticum, Rio das Minas; maximumElevationInMeters: 524; verbatimCoordinates: $3^{\circ} 50^{\prime} 3$ "S, $40^{\circ} 54^{\prime} 18$ "W; samplingProtocol: Manual; verbatimEventDate: 18.ii.13; individualCount: 1; sex: male; lifeStage: adult; recordedBy: Santos, A.P.M. | Takiya, D.M.; identifiedBy: Ângelo Parise Pinto | Marcus Vinicius O. de Almeida; institutionCode: DZRJ; basisOfRecord: PreservedSpecimen

Distribution: Mexico south to Panama. Trinidad and Tobago. Colombia. Venezuela. Guyana. Suriname. French Guiana. Brazil: RR, PA, AM, CE, PE, MT, RO, BA, GO, MG, MS, ES, SP, RJ, SC. Ecuador. Peru. Paraguay. Argentina. 


\section{Genus Erythrodiplax Brauer, 1868}

\section{Erythrodiplax basalis* (Kirby, 1897)}

\section{Material}

a. country: Brazil; stateProvince: Ceará; municipality: Ubajara; locality: Parque Nacional de Ubajara, Trilha Samambaia, Rio Gameleira; maximumElevationInMeters: 874; verbatimCoordinates: $3^{\circ} 50^{\prime 25}$ "S, 4054'19"W; samplingProtocol: Manual; verbatimEventDate: 24.iv.12; individualCount: 1; sex: male; lifeStage: adult; recordedBy: Takiya, D.M. | Cavichioli, R.R.; identifiedBy: Ângelo Parise Pinto; institutionCode: DZRJ; basisOfRecord: PreservedSpecimen

Distribution: Trinidad and Tobago. Colombia. Venezuela. Guyana. Suriname. French Guiana. Brazil: RR, PA, AM, MA, PI!, CE, PE, MT, RO, BA, MG, MS, ES, SP, RJ, RS. Ecuador. Peru. Bolivia. Paraguay. Argentina. Uruguay.

\section{Erythrodiplax castanea (Burmeister, 1839)}

\section{Material}

a. country: Brazil; stateProvince: Ceará; municipality: Ubajara; locality: Parque Nacional de Ubajara, Trilha Samambaia, Rio Gameleira; maximumElevationInMeters: 874; verbatimCoordinates: $3^{\circ} 50^{\prime 2} 2$ "S, $^{\circ} 0^{\circ} 54^{\prime} 19^{\prime \prime W}$; samplingProtocol: Manual; verbatimEventDate: 24.iv.12; individualCount: 1; sex: male; lifeStage: adult; recordedBy: Takiya, D.M. | Cavichioli, R.R.; identifiedBy: Ângelo Parise Pinto; institutionCode: DZRJ; basisOfRecord: PreservedSpecimen

Distribution: Belize. Guatemala. Costa Rica. Trinidad and Tobago. Colombia. Venezuela. Guyana. Suriname. French Guiana. Brazil: PA, AM, CE!, PE, MT, RO, BA, GO, MS, ES, SP, RJ, SC. Ecuador. Peru. Bolivia. Paraguay. Argentina.

Notes: New species record for CE.

\section{Erythrodiplax fusca* (Rambur, 1842)}

\section{Material}

a. country: Brazil; stateProvince: Ceará; municipality: Ubajara; locality: Parque Nacional de Ubajara, Trilha Araticum, Rio das Minas; maximumElevationInMeters: 524; verbatimCoordinates: $3^{\circ} 50^{\prime} 3$ "S, $40^{\circ} 54^{\prime} 18^{\prime \prime} \mathrm{W}$; samplingProtocol: Manual; verbatimEventDate: 18.ii.13; individualCount: 1; sex: male; lifeStage: adult; recordedBy: Santos, A.P.M. | Takiya, D.M.; identifiedBy: Ângelo Parise Pinto; institutionCode: DZRJ; basisOfRecord: PreservedSpecimen

Distribution: USA south to Panama. Trinidad and Tobago. Colombia. Venezuela. Guyana. Suriname. French Guiana. Brazil: PA, AM, MA, PI, CE, RN, PE, MT, RO, BA, GO, MG, MS, ES, SP, RJ, SC, RS. Ecuador. Peru. Bolivia. Paraguay. Argentina. Uruguay. 


\section{Erythrodiplax paraguayensis (Förster, 1904)}

\section{Material}

a. country: Brazil; stateProvince: Ceará; municipality: Ubajara; locality: Parque Nacional de Ubajara, Rio Cafundó, pouco acima da cachoeira; maximumElevationInMeters: 795; verbatimCoordinates: $3^{\circ} 50^{\prime} 13^{\prime \prime} \mathrm{S}, 40^{\circ} 54^{\prime} 35 " \mathrm{~W}$; samplingProtocol: Suspended intercept trap; verbatimEventDate: 10.ii.13; individualCount: 1; sex: female; lifeStage: adult; recordedBy: Câmara, J.T.; identifiedBy: Ângelo Parise Pinto; institutionCode: DZRJ; basisOfRecord: PreservedSpecimen

Distribution: Colombia. Venezuela. Guyana. Suriname. Brazil: RR, MA, CE, MT, MG, MS, SP, RJ, RS. Ecuador. Bolivia. Paraguay. Argentina.

\section{Genus Macrothemis Hagen, 1868}

\section{Macrothemis rupicola Rácenis, 1957}

\section{Material}

a. country: Brazil; stateProvince: Ceará; municipality: Ubajara; locality: Parque Nacional de Ubajara, Trilha Araticum, Rio das Minas; maximumElevationInMeters: 524; verbatimCoordinates: $3^{\circ} 50$ '3"S, 4054'18"W; samplingProtocol: Manual; verbatimEventDate: 18.ii.13; individualCount: 1; sex: male; lifeStage: adult; recordedBy: Santos, A.P.M. | Takiya, D.M.; identifiedBy: Ângelo Parise Pinto; institutionCode: DZRJ; basisOfRecord: PreservedSpecimen

Distribution: Venezuela. French Guiana. Brazil: CE!, MG, RJ.

Notes: New species record for Northeastern Brazil.

\section{Family incertae sedis}

\section{Genus Neocordulia Selys, 1882}

Notes: New genus record for CE.

\section{Neocordulia setifera (Hagen in Selys, 1871)}

\section{Material}

a. country: Brazil; stateProvince: Ceará; municipality: Ubajara; locality: Parque Nacional de Ubajara, Trilha Araticum, Rio das Minas; maximumElevationInMeters: 524; verbatimCoordinates: $3^{\circ} 50^{\prime} 3$ "S, 4054'18"W; samplingProtocol: Manual; verbatimEventDate: 18.ii.13; individualCount: 1; sex: male; lifeStage: adult; recordedBy: Santos, A.P.M. | Takiya, D.M.; identifiedBy: Ângelo Parise Pinto; institutionCode: DZRJ; basisOfRecord: PreservedSpecimen 
Distribution: Brazil: CE!, SP, RJ, PR. Argentina.

Notes: New species record for Northeastern Brazil. See Fig. 23.

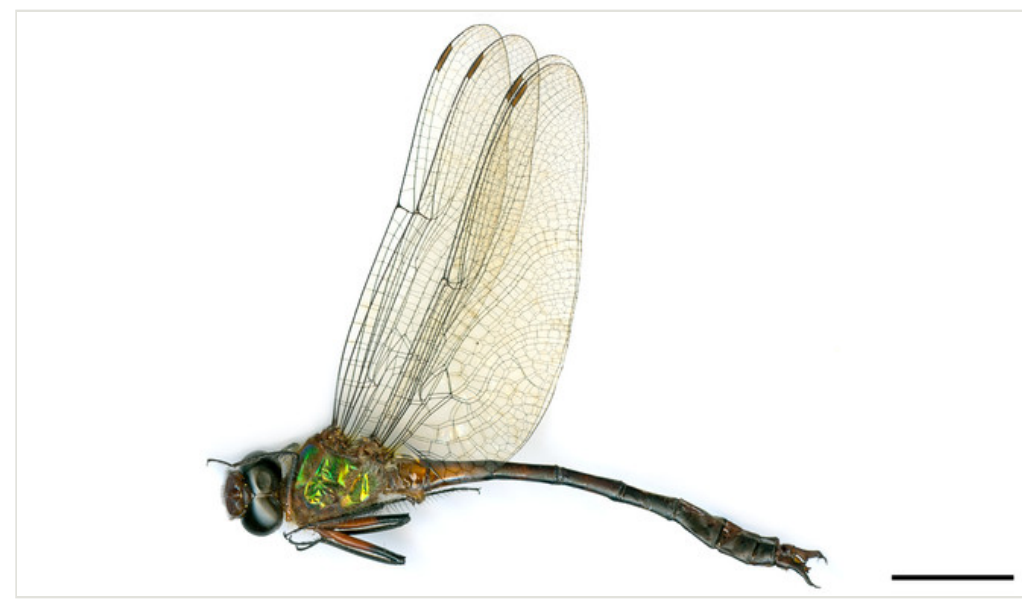

Figure 23.

Lateral habitus of a male of Neocordulia setifera collected at PNU-02 (DZRJ), representing its first record for Northeastern Brazil. Scale bar $=10 \mathrm{~mm}$.

\section{Suborder Zygoptera}

\section{Family Calopterygidae}

\section{Genus Hetaerina Hagen in Selys, 1853}

\section{Hetaerina indeprensa Garrison, 1990}

\section{Materials}

a. country: Brazil; stateProvince: Ceará; municipality: Ubajara; locality: Parque Nacional de Ubajara, Trilha Araticum, Rio das Minas na altura da trilha do teleférico; maximumElevationInMeters: 420; verbatimCoordinates: 349'58"S, 4053'53"W; samplingProtocol: Malaise intercept trap; verbatimEventDate: 14.ii.13; individualCount: 1; sex: male; lifeStage: adult; recordedBy: Rafael, J.A. | Limeira-de-Oliveira, F. I Takiya, D.M. | Santos, A.P.M. | et al.; identifiedBy: Ângelo Parise Pinto; institutionCode: DZRJ; basisOfRecord: PreservedSpecimen

b. country: Brazil; stateProvince: Ceará; municipality: Ubajara; locality: Parque Nacional de Ubajara, Rio das Minas, próximo ao Portão Araticum; maximumElevationInMeters: 328; verbatimCoordinates: 349'32.6"S, 4053'32.8"W; samplingProtocol: Malaise intercept trap; verbatimEventDate: 16.ii.13; individualCount: 1; sex: male; lifeStage: adult; recordedBy: Rafael, J.A. | Limeira-de-Oliveira, F. | Takiya, D.M. | Santos, A.P.M. | et al.; identifiedBy: Ângelo Parise Pinto; institutionCode: DZRJ; basisOfRecord:

PreservedSpecimen 
Distribution: Brazil: PA, CE!.

Notes: New species record for Northeastern Brazil.

\section{Genus Mnesarete Cowley, 1934}

\section{Mnesarete sp. 1}

\section{Material}

a. country: Brazil; stateProvince: Ceará; municipality: Ubajara; locality: Parque Nacional de Ubajara, Trilha Araticum, Rio das Minas; maximumElevationInMeters: 524; verbatimCoordinates: $3^{\circ} 50^{\prime} 3$ "S, $40^{\circ} 54^{\prime} 18 \mathrm{WW}$; samplingProtocol: Manual; verbatimEventDate: 18.ii.13; individualCount: 1; sex: female; lifeStage: adult; recordedBy: Santos, A.P.M. | Takiya, D.M.; identifiedBy: Ângelo Parise Pinto; institutionCode: DZRJ; basisOfRecord: PreservedSpecimen

\section{Family Coenagrionidae}

Genus Acanthagrion Selys, 1876

\section{Acanthagrion aff. quadratum Selys, 1876}

\section{Material}

a. country: Brazil; stateProvince: Ceará; municipality: Ubajara; locality: Parque Nacional de Ubajara, Cachoeira do Cafundó; maximumElevationInMeters: 783; verbatimCoordinates: $3^{\circ} 50^{\prime} 12^{\prime \prime S}, 40^{\circ} 54^{\prime} 35 " \mathrm{~W}$; sampling Protocol: Malaise intercept trap; verbatimEventDate: 18.xi.12; individualCount: 1; sex: female; lifeStage: adult; recordedBy: Limeira-de-Oliveira, F. | Marques, D.W.A.; identifiedBy: Ângelo Parise Pinto; institutionCode: DZRJ; basisOfRecord: PreservedSpecimen

\section{Genus Argia Rambur, 1842}

\section{Argia lilacina Selys, 1865}

\section{Material}

a. country: Brazil; stateProvince: Ceará; municipality: Ubajara; locality: Parque Nacional de Ubajara, Cachoeira do Cafundó; maximumElevationInMeters: 783; verbatimCoordinates: 350'12"S, 4054'35"W; samplingProtocol: Manual; verbatimEventDate: 13.xi.12; individualCount: 1; sex: male; lifeStage: adult; recordedBy: Limeira-de-Oliveira, F. I Marques, D.W.A.; identifiedBy: Ângelo Parise Pinto; institutionCode: DZRJ; basisOfRecord: PreservedSpecimen 
Distribution: Brazil: CE!, TO, MT, GO, MG, MS, ES, SP, RJ. Bolivia. Paraguay. Argentina.

Notes: New species record for Northeastern Brazil.

\section{Argia modesta Selys, 1865}

\section{Materials}

a. $\quad$ country: Brazil; stateProvince: Ceará; municipality: Ubajara; locality: Parque Nacional de Ubajara, Cachoeira do Cafundó; maximumElevationInMeters: 783; verbatimCoordinates: $3^{\circ} 50^{\prime} 12^{\prime \prime S}, 40^{\circ} 54^{\prime} 35 " \mathrm{~W}$; samplingProtocol: Manual; verbatimEventDate: 13.xi.12; individualCount: 1; sex: male; lifeStage: adult; recordedBy: Limeira-de-Oliveira, F. I Marques, D.W.A.; identifiedBy: Rosser W. Garrison; institutionCode: DZRJ; basisOfRecord: PreservedSpecimen

b. country: Brazil; stateProvince: Ceará; municipality: Ubajara; locality: Parque Nacional de Ubajara, Cachoeira do Cafundó; maximumElevationInMeters: 783; verbatimCoordinates: $3^{\circ} 50^{\prime} 12$ "S, 4054'35"W; samplingProtocol: Suspended intercept trap; verbatimEventDate: 16.xii.12; individualCount: 1; sex: male; lifeStage: adult; recordedBy: Limeira-de-Oliveira, F. | Pinto Júnior, J.S.; identifiedBy: Rosser W. Garrison; institutionCode: DZRJ; basisOfRecord: PreservedSpecimen

c. country: Brazil; stateProvince: Ceará; municipality: Ubajara; locality: Parque Nacional de Ubajara, Cachoeira do Cafundó; maximumElevationInMeters: 783; verbatimCoordinates: $3^{\circ} 50^{\prime} 12^{\prime \prime S}$, 4054'35"W; samplingProtocol: Suspended intercept trap; verbatimEventDate: 16.xii.12; individualCount: 1; sex: female; lifeStage: adult; recordedBy: Limeira-de-

Oliveira, F. | Pinto Júnior, J.S.; identifiedBy: Rosser W. Garrison; institutionCode: DZRJ; basisOfRecord: PreservedSpecimen

d. country: Brazil; stateProvince: Ceará; municipality: Ubajara; locality: Parque Nacional de Ubajara, Cachoeira do Cafundó; maximumElevationInMeters: 783; verbatimCoordinates: $3^{\circ} 50^{\prime} 12$ 'S, 4054'35"W; samplingProtocol: Malaise intercept trap; verbatimEventDate: 18.xi.12; individualCount: 1; sex: male; lifeStage: adult; recordedBy: Limeira-de-Oliveira, F. | Marques, D.W.A.; identifiedBy: Rosser W. Garrison; institutionCode: DZRJ; basisOfRecord: PreservedSpecimen

Distribution: Brazil: CE, MG, ES, SP, RJ.

\section{Argia tinctipennis* Selys, 1865}

\section{Materials}

a. country: Brazil; stateProvince: Ceará; municipality: Ubajara; locality: Parque Nacional de Ubajara, Trilha Araticum, Rio das Minas; maximumElevationInMeters: 524; verbatimCoordinates: $3^{\circ} 50$ '3"S, 4054'18"W; sampling Protocol: Manual; verbatimEventDate: 18.ii.13; individualCount: 1; sex: male; lifeStage: adult; recordedBy: Santos, A.P.M. | Takiya, D.M.; identifiedBy: Ângelo Parise Pinto; institutionCode: DZRJ; basisOfRecord: PreservedSpecimen

b. country: Brazil; stateProvince: Ceará; municipality: Ubajara; locality: Parque Nacional de Ubajara, Cachoeira do Cafundó; maximumElevationInMeters: 783; verbatimCoordinates: $3^{\circ} 50^{\prime} 12^{\prime \prime}$ S, 4054'35"W; samplingProtocol: Suspended intercept trap; verbatimEventDate: 1.xii.12; individualCount: 1; sex: female; lifeStage: adult; recordedBy: Limeira-de-Oliveira, 
F. | Pinto Júnior, J.S.; identifiedBy: Rosser W. Garrison; institutionCode: DZRJ; basisOfRecord: PreservedSpecimen

Distribution: Brazil: AM, PI!, CE!, MT, GO, MS. Peru.

Notes: New species record for Northeastern Brazil.

\section{Argia sp. 1}

\section{Materials}

a. country: Brazil; stateProvince: Ceará; municipality: Ubajara; locality: Parque Nacional de Ubajara, Cachoeira do Cafundó; maximumElevationInMeters: 783; verbatimCoordinates: $3^{\circ} 50^{\prime} 12^{\prime \prime S}, 40^{\circ} 54^{\prime} 35 " \mathrm{~W}$; sampling Protocol: Suspended intercept trap; verbatimEventDate: 1.xii.12; individualCount: 1; sex: male; lifeStage: adult; recordedBy: Limeira-de-Oliveira, F. | Pinto Júnior, J.S.; identifiedBy: Rosser W. Garrison; institutionCode: DZRJ; basisOfRecord: PreservedSpecimen

b. $\quad$ country: Brazil; stateProvince: Ceará; municipality: Ubajara; locality: Parque Nacional de Ubajara, Trilha Samambaia, Rio Gameleira; maximumElevationInMeters: 874; verbatimCoordinates: $3^{\circ} 50^{\prime 25}$ "S, 4054'19"W; samplingProtocol: Manual; verbatimEventDate: 24.iv.12; individualCount: 1; sex: female; lifeStage: adult; recordedBy: Takiya, D.M. | Cavichioli, R.R.; identifiedBy: Alcimar Carvalho; institutionCode: DZRJ; basisOfRecord: PreservedSpecimen

\section{Genus Oxyagrion Selys, 1876}

\section{Oxyagrion chapadense Costa, 1978}

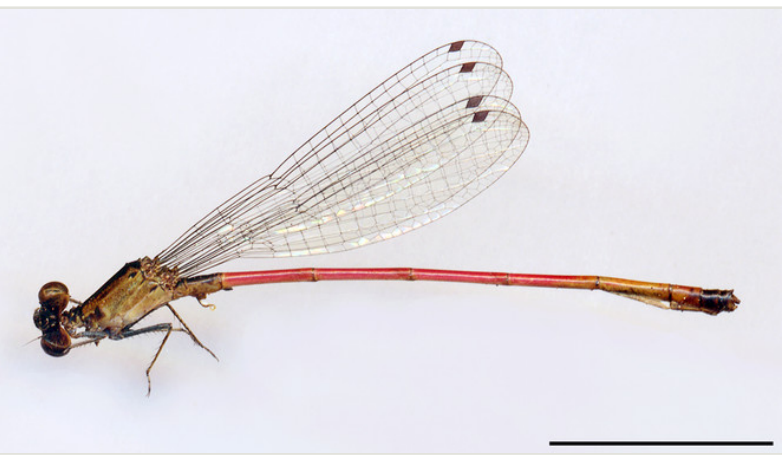

Figure 24.

Lateral habitus of a male melanistic morph of Oxyagrion chapadense collected at PNU-02 (DZRJ). Scale bar $=10 \mathrm{~mm}$.

\section{Material}

a. country: Brazil; stateProvince: Ceará; municipality: Ubajara; locality: Parque Nacional de Ubajara, Trilha Araticum, Rio das Minas; maximumElevationInMeters: 524; verbatimCoordinates: 350'3"S, 4054'18"W; sampling Protocol: Manual; 
verbatimEventDate: 18.ii.13; individualCount: 1; sex: male; lifeStage: adult; recordedBy: Santos, A.P.M. | Takiya, D.M.; identifiedBy: Ângelo Parise Pinto; institutionCode: DZRJ; basisOfRecord: PreservedSpecimen

Distribution: Brazil: CE, MT, BA, GO, MG, MS, SP, PR. Bolivia. Paraguay. Argentina. Uruguay.

Notes: See Fig. 24.

\section{Order Plecoptera}

\section{Family Perlidae}

\section{Genus Anacroneuria Klapálek, 1909}

\section{Anacroneuria calori* Duarte \& Lecci, 2016}

\section{Materials}

a. $\quad$ country: Brazil; stateProvince: Ceará; municipality: Ubajara; locality: Parque Nacional de Ubajara, Trilha Araticum, Rio da Minas abaixo do teleférico; maximumElevationInMeters: 395; verbatimCoordinates: $3^{\circ} 49^{\prime} 43.3^{\prime \prime S}, 40^{\circ} 53^{\prime} 51.5^{\prime \prime}$; samplingProtocol: Malaise intercept trap; verbatimEventDate: 1.ii.13; individualCount: 2; sex: male; lifeStage: adult; recordedBy: Rafael, J.A. | Limeira-de-Oliveira, F. | Takiya, D.M. | Santos, A.P.M | et al.; identifiedBy: Fernanda Avelino-Capistrano; institutionCode: DZRJ; basisOfRecord: PreservedSpecimen

b. country: Brazil; stateProvince: Ceará; municipality: Ubajara; locality: Parque Nacional de Ubajara, Trilha Araticum, Rio da Minas abaixo do teleférico; maximumElevationInMeters: 395; verbatimCoordinates: $3^{\circ} 49^{\prime} 43.3^{\prime \prime} \mathrm{S}, 40^{\circ} 53^{\prime} 51.5^{\prime \prime} \mathrm{W}$; sampling Protocol: Malaise intercept trap; verbatimEventDate: 1.ii.13; individualCount: 2; sex: female; lifeStage: adult; recordedBy: Rafael, J.A. | Limeira-de-Oliveira, F. | Takiya, D.M. | Santos, A.P.M | et al.; identifiedBy: Fernanda Avelino-Capistrano; institutionCode: DZRJ; basisOfRecord: PreservedSpecimen

c. country: Brazil; stateProvince: Ceará; municipality: Ubajara; locality: Parque Nacional de Ubajara, Trilha Samambaia, Rio Gameleira; maximumElevationInMeters: 874; verbatimCoordinates: $3^{\circ} 50^{\prime} 25 " \mathrm{~S}, 40^{\circ} 54^{\prime} 19^{\prime \prime} \mathrm{W}$; samplingProtocol: Pennsylvania light trap; verbatimEventDate: 14.ii.13; individualCount: 2; sex: male; lifeStage: adult; recordedBy: Santos, A.P.M. | Takiya, D.M.; identifiedBy: Fernanda Avelino-Capistrano; institutionCode: DZRJ; basisOfRecord: PreservedSpecimen

d. country: Brazil; stateProvince: Ceará; municipality: Ubajara; locality: Parque Nacional de Ubajara, Trilha Araticum, Rio das Minas; maximumElevationInMeters: 524; verbatimCoordinates: $3^{\circ} 50^{\prime} 3$ 'S, $40^{\circ} 54^{\prime} 18^{\prime \prime} \mathrm{W}$; samplingProtocol: Pennsylvania light trap; verbatimEventDate: 14.ii.13; individualCount: 3; sex: male; lifeStage: adult; recordedBy: Santos, A.P.M. | Takiya, D.M.; identifiedBy: Fernanda Avelino-Capistrano; institutionCode: DZRJ; basisOfRecord: PreservedSpecimen

e. country: Brazil; stateProvince: Ceará; municipality: Ubajara; locality: Parque Nacional de Ubajara, Trilha Araticum, Rio das Minas; maximumElevationInMeters: 524; verbatimCoordinates: $3^{\circ} 50^{\prime} 3$ "S, 4054'18"W; samplingProtocol: Malaise intercept trap; verbatimEventDate: 16.ii.13; individualCount: 1; sex: male; lifeStage: adult; recordedBy: 
Rafael, J.A. | Limeira-de-Oliveira, F. | Takiya, D.M. | Santos, A.P.M | et al.; identifiedBy: Fernanda Avelino-Capistrano; institutionCode: DZRJ; basisOfRecord: PreservedSpecimen

f. country: Brazil; stateProvince: Ceará; municipality: Ubajara; locality: Parque Nacional de Ubajara, Cachoeira do Cafundó; maximumElevationInMeters: 783; verbatimCoordinates: $3^{\circ} 50^{\prime} 12$ 'S, 4054'35"W; samplingProtocol: Manual; verbatimEventDate: 16.ii.13; individualCount: 4; lifeStage: immature; recordedBy: Santos, A.P.M. | Takiya, D.M.; identifiedBy: Fernanda Avelino-Capistrano; institutionCode: DZRJ; basisOfRecord: PreservedSpecimen

g. country: Brazil; stateProvince: Ceará; municipality: Ubajara; locality: Parque Nacional de Ubajara, Portão Neblina; maximumElevationInMeters: 849 ; verbatimCoordinates: $3^{\circ}$ 50'18"S, 4053'54"W; samplingProtocol: Pennsylvania light trap; verbatimEventDate: 17.ii.13; individualCount: 1; sex: male; lifeStage: adult; recordedBy: Santos, A.P.M. | Takiya, D.M.; identifiedBy: Fernanda Avelino-Capistrano; institutionCode: DZRJ; basisOfRecord: PreservedSpecimen

h. country: Brazil; stateProvince: Ceará; municipality: Ubajara; locality: Parque Nacional de Ubajara, Trilha Araticum, Rio das Minas; maximumElevationInMeters: 524; verbatimCoordinates: $3^{\circ} 50^{\prime} 3$ "S, $40^{\circ} 54^{\prime} 18^{\prime \prime} \mathrm{W}$; samplingProtocol: Manual; verbatimEventDate: 19.ii.13; individualCount: 1; lifeStage: immature; recordedBy: Santos, A.P.M. | Takiya, D.M.; identifiedBy: Fernanda Avelino-Capistrano; institutionCode: DZRJ; basisOfRecord: PreservedSpecimen

i. country: Brazil; stateProvince: Ceará; municipality: Ubajara; locality: Parque Nacional de Ubajara, Trilha Araticum, Rio das Minas na altura da trilha do teleférico; maximumElevationInMeters: 420; verbatimCoordinates: $3^{\circ} 49^{\prime} 58^{\prime \prime S}, 40^{\circ} 53^{\prime} 53^{\prime \prime} \mathrm{W}$; samplingProtocol: Malaise intercept trap; verbatimEventDate: 20.iv.12; individualCount: 7; sex: male; lifeStage: adult; recordedBy: Rafael, J.A. | Limeira-de-Oliveira, F. | Takiya, D.M. | et al.; identifiedBy: Fernanda Avelino-Capistrano; institutionCode: DZRJ; basisOfRecord: PreservedSpecimen

j. country: Brazil; stateProvince: Ceará; municipality: Ubajara; locality: Parque Nacional de Ubajara, Trilha Samambaia, Rio Gameleira; maximumElevationInMeters: 874; verbatimCoordinates: $3^{\circ} 50^{\prime} 25$ "S, $40^{\circ} 54^{\prime} 19$ "W; sampling Protocol: Pennsylvania light trap; verbatimEventDate: 21.iv.12; individualCount: 1; sex: female; lifeStage: adult; recordedBy: Takiya, D.M. | Câmara, J.T.; identifiedBy: Fernanda Avelino-Capistrano; institutionCode: DZRJ; basisOfRecord: PreservedSpecimen

k. country: Brazil; stateProvince: Ceará; municipality: Ubajara; locality: Parque Nacional de Ubajara, Trilha Araticum, Rio das Minas; maximumElevationInMeters: 524; verbatimCoordinates: $3^{\circ} 50^{\prime} 3$ "S, $40^{\circ} 54^{\prime} 18$ "W; samplingProtocol: Pennsylvania light trap; verbatimEventDate: 22.iv.12; individualCount: 1; sex: female; lifeStage: adult; recordedBy: Takiya, D.M. | Somavilla, A.; identifiedBy: Fernanda Avelino-Capistrano; institutionCode: DZRJ; basisOfRecord: PreservedSpecimen

I. country: Brazil; stateProvince: Ceará; municipality: Ubajara; locality: Parque Nacional de Ubajara, Trilha Araticum, Rio Cafundó; maximumElevationInMeters: 753; verbatimCoordinates: $3^{\circ} 50^{\prime} 12$ "S, 4054'31"W; samplingProtocol: Manual; verbatimEventDate: 23.iv.12; individualCount: 1; lifeStage: immature; recordedBy: Takiya, D.M.; identifiedBy: Fernanda Avelino-Capistrano; institutionCode: DZRJ; basisOfRecord: PreservedSpecimen

m. country: Brazil; stateProvince: Ceará; municipality: Ubajara; locality: Parque Nacional de Ubajara, Trilha Araticum, Rio das Minas na altura da trilha do teleférico; maximumElevationInMeters: 420; verbatimCoordinates: $3^{\circ} 49^{\prime} 58^{\prime \prime S}, 40^{\circ} 53^{\prime} 53 " \mathrm{~W}$; samplingProtocol: Malaise intercept trap; verbatimEventDate: 23.iv.12; individualCount: 7; 
sex: female; lifeStage: adult; recordedBy: Rafael, J.A. | Limeira-de-Oliveira, F. | Takiya, D.M. | et al.; identifiedBy: Fernanda Avelino-Capistrano; institutionCode: DZRJ; basisOfRecord: PreservedSpecimen

n. country: Brazil; stateProvince: Ceará; municipality: Ubajara; locality: Parque Nacional de Ubajara, Cachoeira do Cafundó; maximumElevationInMeters: 783; verbatimCoordinates: $3^{\circ} 50^{\prime} 12^{\prime \prime S}, 40^{\circ} 54^{\prime} 35 " \mathrm{~W}$; sampling Protocol: Pennsylvania light trap; verbatimEventDate: 24.iv.12; individualCount: 1; sex: male; lifeStage: adult; recordedBy: Takiya, D.M. | Rafael, J.A.; identifiedBy: Fernanda Avelino-Capistrano; institutionCode: DZRJ; basisOfRecord: PreservedSpecimen

Distribution: Brazil: PI!, CE.

Notes: See Fig. 25.

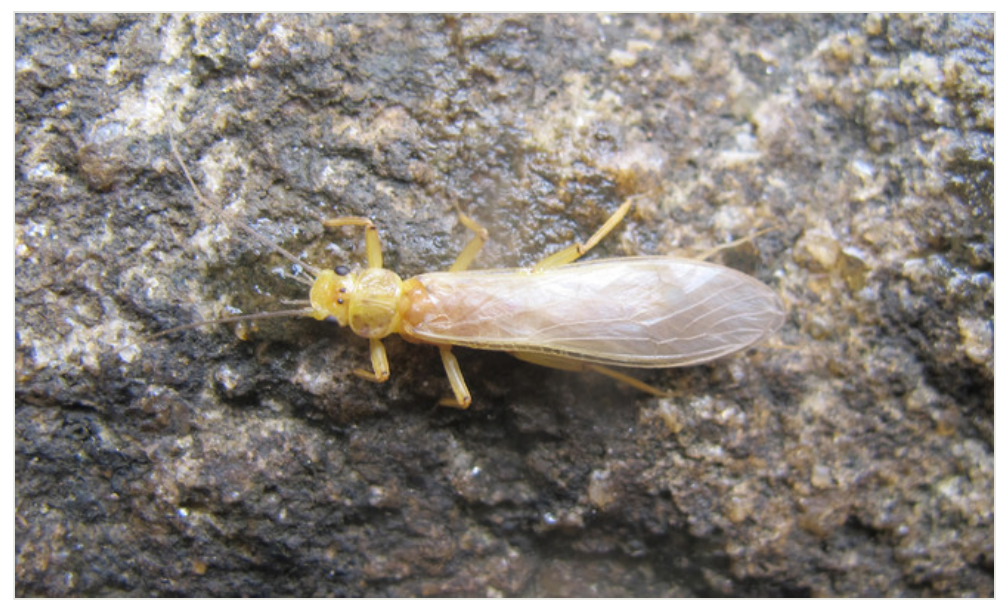

Figure 25.

Teneral adult specimen of Anacroneuria calori collected at PNU-02. This species was also recorded from PNSC, representing the first record of Plecoptera for Piauí.

\section{Order Trichoptera}

\section{Family Calamoceratidae}

\section{Genus Phylloicus Müller, 1880}

\section{Phylloicus obliquus Navás, 1931}

\section{Materials}

a. country: Brazil; stateProvince: Ceará; municipality: Ubajara; locality: Parque Nacional de Ubajara, Rio Cafundó, pouco acima da cachoeira; maximumElevationInMeters: 795; verbatimCoordinates: $3^{\circ} 50^{\prime} 13^{\prime \prime S}, 40^{\circ} 54^{\prime} 35 " \mathrm{~W}$; samplingProtocol: Malaise intercept trap; verbatimEventDate: 1.xii.12; individualCount: 3; sex: male; lifeStage: adult; recordedBy: 
Limeira-de-Oliveira | et al.; identifiedBy: Allan Paulo Moreira dos Santos; institutionCode: DZRJ; basisOfRecord: PreservedSpecimen

b. country: Brazil; stateProvince: Ceará; municipality: Ubajara; locality: Parque Nacional de Ubajara, Rio Cafundó, pouco acima da cachoeira; maximumElevationInMeters: 795; verbatimCoordinates: $3^{\circ} 50^{\prime} 13^{\prime \prime} \mathrm{S}, 40^{\circ} 54^{\prime} 35 " \mathrm{~W}$; samplingProtocol: Malaise intercept trap; verbatimEventDate: 13.xi.12; individualCount: 1; sex: male; lifeStage: adult; recordedBy: Limeira-de-Oliveira | et al.; identifiedBy: Allan Paulo Moreira dos Santos; institutionCode: DZRJ; basisOfRecord: PreservedSpecimen

C. country: Brazil; stateProvince: Ceará; municipality: Ubajara; locality: Parque Nacional de Ubajara, Rio Cafundó, pouco acima da cachoeira; maximumElevationInMeters: 795; verbatimCoordinates: $3^{\circ} 50^{\prime} 13^{\prime \prime} \mathrm{S}, 40^{\circ} 54^{\prime} 35 " \mathrm{~W}$; samplingProtocol: Malaise intercept trap; verbatimEventDate: 13.xi.12; individualCount: 1; sex: female; lifeStage: adult; recordedBy: Limeira-de-Oliveira | et al.; identifiedBy: Allan Paulo Moreira dos Santos; institutionCode: DZRJ; basisOfRecord: PreservedSpecimen

d. country: Brazil; stateProvince: Ceará; municipality: Ubajara; locality: Parque Nacional de Ubajara, Rio Cafundó, pouco acima da cachoeira; maximumElevationInMeters: 795; verbatimCoordinates: $3^{\circ} 50^{\prime} 13^{\prime \prime S}, 40^{\circ} 54^{\prime} 35 " \mathrm{~W}$; samplingProtocol: Malaise intercept trap; verbatimEventDate: 13.xi.12; individualCount: 2; sex: male; lifeStage: adult; recordedBy: Limeira-de-Oliveira | et al.; identifiedBy: Allan Paulo Moreira dos Santos; institutionCode: DZRJ; basisOfRecord: PreservedSpecimen

e. country: Brazil; stateProvince: Ceará; municipality: Ubajara; locality: Parque Nacional de Ubajara, Rio Cafundó, pouco acima da cachoeira; maximumElevationInMeters: 795; verbatimCoordinates: $3^{\circ} 50^{\prime} 13^{\prime \prime S}, 40^{\circ} 54^{\prime} 35 " \mathrm{~W}$; samplingProtocol: Malaise intercept trap; verbatimEventDate: 13.xi.12; individualCount: 1; sex: female; lifeStage: adult; recordedBy: Limeira-de-Oliveira | et al.; identifiedBy: Allan Paulo Moreira dos Santos; institutionCode: DZRJ; basisOfRecord: PreservedSpecimen

f. country: Brazil; stateProvince: Ceará; municipality: Ubajara; locality: Parque Nacional de Ubajara, Trilha Araticum, Rio das Minas na altura da trilha do teleférico; maximumElevationInMeters: 420; verbatimCoordinates: $3^{\circ} 49^{\prime} 58$ "S, 4053'53"W; samplingProtocol: Malaise intercept trap; verbatimEventDate: 14.ii.13; individualCount: 1; sex: male; lifeStage: adult; recordedBy: Rafael, J.A. | Limeira-de-Oliveira, F. | Takiya, D.M. | Santos, A.P.M. | et al.; identifiedBy: Allan Paulo Moreira dos Santos; institutionCode: DZRJ; basisOfRecord: PreservedSpecimen

g. country: Brazil; stateProvince: Ceará; municipality: Ubajara; locality: Parque Nacional de Ubajara, Trilha Araticum, Rio das Minas na altura da trilha do teleférico; maximumElevationInMeters: 420; verbatimCoordinates: $3^{\circ} 49^{\prime} 58^{\prime \prime S}, 40^{\circ} 53^{\prime} 53^{\prime \prime} \mathrm{W}$; sampling Protocol: Malaise intercept trap; verbatimEventDate: 14.ii.13; individualCount: 1; sex: female; lifeStage: adult; recordedBy: Rafael, J.A. | Limeira-de-Oliveira, F. | Takiya, D.M. | Santos, A.P.M. | et al.; identifiedBy: Allan Paulo Moreira dos Santos; institutionCode: DZRJ; basisOfRecord: PreservedSpecimen

h. country: Brazil; stateProvince: Ceará; municipality: Ubajara; locality: Parque Nacional de Ubajara, Trilha Samambaia, Rio Gameleira; maximumElevationInMeters: 874; verbatimCoordinates: $3^{\circ} 50^{\prime} 25^{\prime \prime S}, 40^{\circ} 54^{\prime} 19 " \mathrm{~W}$; samplingProtocol: Malaise intercept trap; verbatimEventDate: 20.iv.12; individualCount: 13; sex: male; lifeStage: adult; recordedBy: Rafael, J.A. | Limeira-de-Oliveira, F. | Takiya, D.M. | et al.; identifiedBy: Allan Paulo Moreira dos Santos; institutionCode: DZRJ; basisOfRecord: PreservedSpecimen

i. country: Brazil; stateProvince: Ceará; municipality: Ubajara; locality: Parque Nacional de Ubajara, Trilha Samambaia, Rio Gameleira; maximumElevationInMeters: 874; verbatimCoordinates: $3^{\circ} 50^{\prime} 25^{\prime \prime} \mathrm{S}, 40^{\circ} 54^{\prime} 19^{\prime \prime} \mathrm{W}$; samplingProtocol: Malaise intercept trap; verbatimEventDate: 20.iv.12; individualCount: 1; sex: female; lifeStage: adult; recordedBy: 
Rafael, J.A. | Limeira-de-Oliveira, F. | Takiya, D.M. | et al.; identifiedBy: Allan Paulo Moreira dos Santos; institutionCode: DZRJ; basisOfRecord: PreservedSpecimen

j. country: Brazil; stateProvince: Ceará; municipality: Ubajara; locality: Parque Nacional de Ubajara, Rio Cafundó, pouco acima da cachoeira; maximumElevationInMeters: 795; verbatimCoordinates: $3^{\circ} 50^{\prime} 13^{\prime \prime} \mathrm{S}, 40^{\circ} 54^{\prime} 35^{\prime \prime} \mathrm{W}$; samplingProtocol: Malaise intercept trap; verbatimEventDate: 21.iv.12; individualCount: 6; sex: male; lifeStage: adult; recordedBy: Rafael, J.A. | Limeira-de-Oliveira, F. | Takiya, D.M. | et al.; identifiedBy: Allan Paulo Moreira dos Santos; institutionCode: DZRJ; basisOfRecord: PreservedSpecimen

k. country: Brazil; stateProvince: Ceará; municipality: Ubajara; locality: Parque Nacional de Ubajara, Rio Cafundó, pouco acima da cachoeira; maximumElevationInMeters: 795; verbatimCoordinates: $3^{\circ} 50^{\prime} 13^{\prime \prime} \mathrm{S}, 40^{\circ} 54^{\prime} 35 " \mathrm{~W}$; samplingProtocol: Pennsylvania light trap; verbatimEventDate: 24.iv.12; individualCount: 1; sex: male; lifeStage: adult; recordedBy: Takiya, D.M. | Rafael, J.A.; identifiedBy: Allan Paulo Moreira dos Santos; institutionCode: DZRJ; basisOfRecord: PreservedSpecimen

Distribution: Brazil: CE, BA, MG, RJ, SC.

\section{Phylloicus pirapo Prather, 2003}

\section{Materials}

a. country: Brazil; stateProvince: Ceará; municipality: Ubajara; locality: Parque Nacional de Ubajara, Rio Cafundó, pouco acima da cachoeira; maximumElevationInMeters: 795; verbatimCoordinates: $3^{\circ} 50^{\prime} 13^{\prime \prime}$ S, $40^{\circ} 54^{\prime} 35 " \mathrm{~W}$; samplingProtocol: Suspended intercept trap; verbatimEventDate: 1.ii.13; individualCount: 1; sex: male; lifeStage: adult; recordedBy: Limeira-de-Oliveira | Rafael, J.A. | et al.; identifiedBy: Allan Paulo Moreira dos Santos; institutionCode: DZRJ; basisOfRecord: PreservedSpecimen

b. $\quad$ country: Brazil; stateProvince: Ceará; municipality: Ubajara; locality: Parque Nacional de Ubajara, Rio Cafundó, pouco acima da cachoeira; maximumElevationInMeters: 795; verbatimCoordinates: $3^{\circ} 50^{\prime} 13$ "S, 4054'35"W; samplingProtocol: Suspended intercept trap; verbatimEventDate: 1.ii.13; individualCount: 2; sex: female; lifeStage: adult; recordedBy: Limeira-de-Oliveira | Rafael, J.A. | et al.; identifiedBy: Allan Paulo Moreira dos Santos; institutionCode: DZRJ; basisOfRecord: PreservedSpecimen

c. country: Brazil; stateProvince: Ceará; municipality: Ubajara; locality: Parque Nacional de Ubajara, Rio Cafundó, pouco acima da cachoeira; maximumElevationInMeters: 795; verbatimCoordinates: $3^{\circ} 50^{\prime} 13^{\prime \prime} \mathrm{S}, 40^{\circ} 54^{\prime} 35 \mathrm{~W} \mathrm{~W}$; samplingProtocol: Malaise intercept trap; verbatimEventDate: 1.ii.13; individualCount: 2; sex: male; lifeStage: adult; recordedBy: Limeira-de-Oliveira | et al.; identifiedBy: Allan Paulo Moreira dos Santos; institutionCode: DZRJ; basisOfRecord: PreservedSpecimen

d. country: Brazil; stateProvince: Ceará; municipality: Ubajara; locality: Parque Nacional de Ubajara, Rio Cafundó, pouco acima da cachoeira; maximumElevationInMeters: 795; verbatimCoordinates: $3^{\circ} 50^{\prime} 13^{\prime \prime} \mathrm{S}, 40^{\circ} 54^{\prime} 35 \mathrm{~W} \mathrm{~W}$; samplingProtocol: Malaise intercept trap; verbatimEventDate: 1.ii.13; individualCount: 3; sex: female; lifeStage: adult; recordedBy: Limeira-de-Oliveira | et al.; identifiedBy: Allan Paulo Moreira dos Santos; institutionCode: DZRJ; basisOfRecord: PreservedSpecimen

e. $\quad$ country: Brazil; stateProvince: Ceará; municipality: Ubajara; locality: Parque Nacional de Ubajara, Rio Cafundó, pouco acima da cachoeira; maximumElevationInMeters: 795; verbatimCoordinates: $3^{\circ} 50^{\prime} 13^{\prime \prime S}, 40^{\circ} 54^{\prime} 35 \mathrm{WW}$; samplingProtocol: Malaise intercept trap; verbatimEventDate: 1.xii.12; individualCount: 1; sex: female; lifeStage: adult; recordedBy: 
Limeira-de-Oliveira | et al.; identifiedBy: Allan Paulo Moreira dos Santos; institutionCode: DZRJ; basisOfRecord: PreservedSpecimen

f. country: Brazil; stateProvince: Ceará; municipality: Ubajara; locality: Parque Nacional de Ubajara, Trilha Samambaia, Rio Gameleira; maximumElevationInMeters: 874; verbatimCoordinates: $3^{\circ} 50^{\prime 2} 25^{\prime \prime}$ S, $40^{\circ} 54^{\prime} 19^{\prime \prime} \mathrm{W}$; samplingProtocol: Malaise intercept trap; verbatimEventDate: 14.ii.13; individualCount: 1; sex: female; lifeStage: adult; recordedBy: Rafael, J.A. | Limeira-de-Oliveira, F. | Takiya, D.M. | Santos, A.P.M. | et al.; identifiedBy: Allan Paulo Moreira dos Santos; institutionCode: DZRJ; basisOfRecord: PreservedSpecimen

g. country: Brazil; stateProvince: Ceará; municipality: Ubajara; locality: Parque Nacional de Ubajara, Trilha Araticum, Rio das Minas na altura da trilha do teleférico; maximumElevationInMeters: 420; verbatimCoordinates: $3^{\circ} 49^{\prime} 58^{\prime \prime S}, 40^{\circ} 53^{\prime} 53^{\prime \prime} \mathrm{W}$; samplingProtocol: Malaise intercept trap; verbatimEventDate: 14.ii.13; individualCount: 1; sex: male; lifeStage: adult; recordedBy: Rafael, J.A. | Limeira-de-Oliveira, F. | Takiya, D.M. | Santos, A.P.M. | et al.; identifiedBy: Allan Paulo Moreira dos Santos; institutionCode: DZRJ; basisOfRecord: PreservedSpecimen

h. country: Brazil; stateProvince: Ceará; municipality: Ubajara; locality: Parque Nacional de Ubajara, Trilha Araticum, Rio das Minas na altura da trilha do teleférico; maximumElevationInMeters: 420; verbatimCoordinates: 349'58"S, 4053'53"W; samplingProtocol: Malaise intercept trap; verbatimEventDate: 14.ii.13; individualCount: 1; sex: female; lifeStage: adult; recordedBy: Rafael, J.A. | Limeira-de-Oliveira, F. | Takiya, D.M. | Santos, A.P.M. | et al.; identifiedBy: Allan Paulo Moreira dos Santos; institutionCode: DZRJ; basisOfRecord: PreservedSpecimen

i. country: Brazil; stateProvince: Ceará; municipality: Ubajara; locality: Parque Nacional de Ubajara, Trilha Araticum, Rio das Minas na altura da trilha do teleférico; maximumElevationInMeters: 420; verbatimCoordinates: 349'58"S, 4053'53"W; samplingProtocol: Malaise intercept trap; verbatimEventDate: 14.ii.13; individualCount: 12; sex: male; lifeStage: adult; recordedBy: Rafael, J.A. | Limeira-de-Oliveira, F. | Takiya, D.M. | Santos, A.P.M. | et al.; identifiedBy: Allan Paulo Moreira dos Santos; institutionCode: DZRJ; basisOfRecord: PreservedSpecimen

j. country: Brazil; stateProvince: Ceará; municipality: Ubajara; locality: Parque Nacional de Ubajara, Trilha Araticum, Rio das Minas na altura da trilha do teleférico; maximumElevationInMeters: 420; verbatimCoordinates: $3^{\circ} 49^{\prime} 58^{\prime \prime} \mathrm{S}$, 4053'53"W; samplingProtocol: Malaise intercept trap; verbatimEventDate: 14.ii.13; individualCount: 1; sex: female; lifeStage: adult; recordedBy: Rafael, J.A. | Limeira-de-Oliveira, F. | Takiya, D.M. | Santos, A.P.M. | et al.; identifiedBy: Allan Paulo Moreira dos Santos; institutionCode: DZRJ; basisOfRecord: PreservedSpecimen

k. country: Brazil; stateProvince: Ceará; municipality: Ubajara; locality: Parque Nacional de Ubajara, Trilha Araticum, Rio das Minas na altura da trilha do teleférico; maximumElevationInMeters: 420; verbatimCoordinates: $3^{\circ} 49^{\prime} 58$ "S, 4053'53"W; samplingProtocol: Malaise intercept trap; verbatimEventDate: 14.ii.13; individualCount: 15; sex: male; lifeStage: adult; recordedBy: Rafael, J.A. | Limeira-de-Oliveira, F. | Takiya, D.M. | Santos, A.P.M. | et al.; identifiedBy: Allan Paulo Moreira dos Santos; institutionCode: DZRJ; basisOfRecord: PreservedSpecimen

I. country: Brazil; stateProvince: Ceará; municipality: Ubajara; locality: Parque Nacional de Ubajara, Trilha Araticum, Rio das Minas na altura da trilha do teleférico; maximumElevationInMeters: 420; verbatimCoordinates: $3^{\circ} 49^{\prime} 58^{\prime \prime S}, 40^{\circ} 53^{\prime} 53^{\prime \prime} \mathrm{W}$; samplingProtocol: Malaise intercept trap; verbatimEventDate: 14.ii.13; individualCount: 12; sex: female; lifeStage: adult; recordedBy: Rafael, J.A. | Limeira-de-Oliveira, F. | Takiya, 
D.M. | Santos, A.P.M. | et al.; identifiedBy: Allan Paulo Moreira dos Santos; institutionCode: DZRJ; basisOfRecord: PreservedSpecimen

m. country: Brazil; stateProvince: Ceará; municipality: Ubajara; locality: Parque Nacional de Ubajara, Rio das Minas, próximo ao Portão Araticum; maximumElevationInMeters: 328; verbatimCoordinates: $3^{\circ} 49^{\prime} 32.6^{\prime \prime S}, 40^{\circ} 53^{\prime} 32.8^{\prime \prime W}$; samplingProtocol: Malaise intercept trap; verbatimEventDate: 14.ii.13; individualCount: 1; sex: male; lifeStage: adult; recordedBy: Rafael, J.A. | Limeira-de-Oliveira, F. | Takiya, D.M. | Santos, A.P.M. | et al.; identifiedBy: Allan Paulo Moreira dos Santos; institutionCode: DZRJ; basisOfRecord: PreservedSpecimen

n. country: Brazil; stateProvince: Ceará; municipality: Ubajara; locality: Parque Nacional de Ubajara, Rio das Minas, próximo ao Portão Araticum; maximumElevationInMeters: 328; verbatimCoordinates: $3^{\circ} 49^{\prime} 32.6^{\prime \prime} \mathrm{S}, 40^{\circ} 53^{\prime} 32.8^{\prime \prime} \mathrm{W}$; samplingProtocol: Malaise intercept trap; verbatimEventDate: 14.ii.13; individualCount: 2; sex: female; lifeStage: adult; recordedBy: Rafael, J.A. | Limeira-de-Oliveira, F. | Takiya, D.M. | Santos, A.P.M. | et al.; identifiedBy: Allan Paulo Moreira dos Santos; institutionCode: DZRJ; basisOfRecord: PreservedSpecimen

o. country: Brazil; stateProvince: Ceará; municipality: Ubajara; locality: Parque Nacional de Ubajara, Rio das Minas, próximo ao Portão Araticum; maximumElevationInMeters: 328; verbatimCoordinates: $3^{\circ} 49^{\prime} 32.6^{\prime \prime S}, 40^{\circ} 53^{\prime} 32.8^{\prime \prime W}$; samplingProtocol: Malaise intercept trap; verbatimEventDate: 14.ii.13; individualCount: 5; sex: male; lifeStage: adult; recordedBy: Rafael, J.A. | Limeira-de-Oliveira, F. | Takiya, D.M. | Santos, A.P.M. | et al.; identifiedBy: Allan Paulo Moreira dos Santos; institutionCode: DZRJ; basisOfRecord: PreservedSpecimen

p. country: Brazil; stateProvince: Ceará; municipality: Ubajara; locality: Parque Nacional de Ubajara, Rio das Minas, próximo ao Portão Araticum; maximumElevationInMeters: 328; verbatimCoordinates: $3^{\circ} 49^{\prime} 32.6^{\prime \prime S}, 40^{\circ} 53^{\prime} 32.8^{\prime \prime W}$; samplingProtocol: Malaise intercept trap; verbatimEventDate: 14.ii.13; individualCount: 6; sex: female; lifeStage: adult; recordedBy: Rafael, J.A. | Limeira-de-Oliveira, F. | Takiya, D.M. | Santos, A.P.M. | et al.; identifiedBy: Allan Paulo Moreira dos Santos; institutionCode: DZRJ; basisOfRecord: PreservedSpecimen

q. $\quad$ country: Brazil; stateProvince: Ceará; municipality: Ubajara; locality: Parque Nacional de Ubajara, Rio das Minas, próximo ao Portão Araticum; maximumElevationInMeters: 328; verbatimCoordinates: $3^{\circ} 49^{\prime} 32.6^{\prime \prime}$ S, 40 $43^{\circ} 32.8^{\prime \prime W}$; samplingProtocol: Malaise intercept trap; verbatimEventDate: 14.ii.13; individualCount: 6; sex: male; lifeStage: adult; recordedBy: Rafael, J.A. | Limeira-de-Oliveira, F. | Takiya, D.M. | Santos, A.P.M. | et al.; identifiedBy: Allan Paulo Moreira dos Santos; institutionCode: DZRJ; basisOfRecord: PreservedSpecimen

r. country: Brazil; stateProvince: Ceará; municipality: Ubajara; locality: Parque Nacional de Ubajara, Rio das Minas, próximo ao Portão Araticum; maximumElevationInMeters: 328; verbatimCoordinates: $3^{\circ} 49^{\prime} 32.6^{\prime \prime S}, 40^{\circ} 53^{\prime} 32.8^{\prime \prime W}$; samplingProtocol: Malaise intercept trap; verbatimEventDate: 14.ii.13; individualCount: 14; sex: female; lifeStage: adult; recordedBy: Rafael, J.A. | Limeira-de-Oliveira, F. | Takiya, D.M. | Santos, A.P.M. | et al.; identifiedBy: Allan Paulo Moreira dos Santos; institutionCode: DZRJ; basisOfRecord: PreservedSpecimen

s. $\quad$ country: Brazil; stateProvince: Ceará; municipality: Ubajara; locality: Parque Nacional de Ubajara, Trilha Samambaia, Rio Gameleira; maximumElevationInMeters: 874; verbatimCoordinates: $3^{\circ} 50^{\prime} 25^{\prime \prime S}, 40^{\circ} 54^{\prime} 19^{\prime \prime W}$; samplingProtocol: Malaise intercept trap; verbatimEventDate: 17.ii.13; individualCount: 2; sex: male; lifeStage: adult; recordedBy: Rafael, J.A. | Limeira-de-Oliveira, F. | Takiya, D.M. | Santos, A.P.M. | et al.; identifiedBy: 
Allan Paulo Moreira dos Santos; institutionCode: DZRJ; basisOfRecord: PreservedSpecimen

t. country: Brazil; stateProvince: Ceará; municipality: Ubajara; locality: Parque Nacional de Ubajara, Trilha Samambaia, Rio Gameleira; maximumElevationInMeters: 874; verbatimCoordinates: $3^{\circ} 50^{\prime} 25^{\prime \prime} \mathrm{S}, 40^{\circ} 54^{\prime} 19^{\prime \prime W}$; samplingProtocol: Malaise intercept trap; verbatimEventDate: 17.ii.13; individualCount: 2; sex: female; lifeStage: adult; recordedBy: Rafael, J.A. | Limeira-de-Oliveira, F. | Takiya, D.M. | Santos, A.P.M. | et al.; identifiedBy: Allan Paulo Moreira dos Santos; institutionCode: DZRJ; basisOfRecord: PreservedSpecimen

u. country: Brazil; stateProvince: Ceará; municipality: Ubajara; locality: Parque Nacional de Ubajara, Trilha Araticum, Rio das Minas; maximumElevationInMeters: 524; verbatimCoordinates: $3^{\circ} 50^{\prime} 3$ "S, $40^{\circ} 54^{\prime} 18 \mathrm{~W}$; samplingProtocol: Malaise intercept trap; verbatimEventDate: 17.ii.13; individualCount: 1; sex: male; lifeStage: adult; recordedBy: Rafael, J.A. | Limeira-de-Oliveira, F. | Takiya, D.M. | Santos, A.P.M. | et al.; identifiedBy: Allan Paulo Moreira dos Santos; institutionCode: DZRJ; basisOfRecord: PreservedSpecimen

v. country: Brazil; stateProvince: Ceará; municipality: Ubajara; locality: Parque Nacional de Ubajara, Trilha Araticum, Rio das Minas; maximumElevationInMeters: 524; verbatimCoordinates: $3^{\circ} 50^{\prime} 3$ "S, $40^{\circ} 54^{\prime} 18 \mathrm{~W}$; samplingProtocol: Malaise intercept trap; verbatimEventDate: 17.ii.13; individualCount: 1; sex: female; lifeStage: adult; recordedBy: Rafael, J.A. | Limeira-de-Oliveira, F. | Takiya, D.M. | Santos, A.P.M. | et al.; identifiedBy: Allan Paulo Moreira dos Santos; institutionCode: DZRJ; basisOfRecord: PreservedSpecimen

w. country: Brazil; stateProvince: Ceará; municipality: Ubajara; locality: Parque Nacional de Ubajara, Rio das Minas, próximo ao Portão Araticum; maximumElevationInMeters: 328; verbatimCoordinates: $3^{\circ} 49^{\prime} 32.6^{\prime \prime S}, 40^{\circ} 53^{\prime} 32.8^{\prime \prime W}$; samplingProtocol: Malaise intercept trap; verbatimEventDate: 17.ii.13; individualCount: 3; sex: male; lifeStage: adult; recordedBy: Rafael, J.A. | Limeira-de-Oliveira, F. | Takiya, D.M. | Santos, A.P.M. | et al.; identifiedBy: Allan Paulo Moreira dos Santos; institutionCode: DZRJ; basisOfRecord: PreservedSpecimen

x. country: Brazil; stateProvince: Ceará; municipality: Ubajara; locality: Parque Nacional de Ubajara, Rio Cafundó, pouco acima da cachoeira; maximumElevationInMeters: 795; verbatimCoordinates: $3^{\circ} 50^{\prime} 13^{\prime \prime S}, 40^{\circ} 54^{\prime} 35^{\prime \prime W}$; samplingProtocol: Suspended intercept trap; verbatimEventDate: 19.i.13; individualCount: 1; sex: male; lifeStage: adult; recordedBy: Rafael, J.A. | Limeira-de-Oliveira, F. | et al.; identifiedBy: Allan Paulo Moreira dos Santos; institutionCode: DZRJ; basisOfRecord: PreservedSpecimen

y. country: Brazil; stateProvince: Ceará; municipality: Ubajara; locality: Parque Nacional de Ubajara, Rio Cafundó, pouco acima da cachoeira; maximumElevationInMeters: 795; verbatimCoordinates: $3^{\circ} 50^{\prime} 13^{\prime \prime} \mathrm{S}, 40^{\circ} 54^{\prime} 35^{\prime \prime W}$; samplingProtocol: Suspended intercept trap; verbatimEventDate: 19.i.13; individualCount: 1; sex: male; lifeStage: adult; recordedBy: Rafael, J.A. | Limeira-de-Oliveira, F. | et al.; identifiedBy: Allan Paulo Moreira dos Santos; institutionCode: DZRJ; basisOfRecord: PreservedSpecimen

z. country: Brazil; stateProvince: Ceará; municipality: Ubajara; locality: Parque Nacional de Ubajara, Trilha Samambaia, Rio Gameleira; maximumElevationInMeters: 874; verbatimCoordinates: $3^{\circ} 50^{\prime} 25^{\prime \prime S}, 40^{\circ} 54^{\prime} 19^{\prime \prime W}$; samplingProtocol: Malaise intercept trap; verbatimEventDate: 20.iv.12; individualCount: 2; sex: female; lifeStage: adult; recordedBy: Rafael, J.A. | Limeira-de-Oliveira, F. | Takiya, D.M. | et al.; identifiedBy: Allan Paulo Moreira dos Santos; institutionCode: DZRJ; basisOfRecord: PreservedSpecimen

aa. country: Brazil; stateProvince: Ceará; municipality: Ubajara; locality: Parque Nacional de Ubajara, Trilha Araticum, Rio das Minas na altura da trilha do teleférico; 
maximumElevationInMeters: 420; verbatimCoordinates: $3^{\circ} 49^{\prime} 58^{\prime \prime S}, 40^{\circ} 53^{\prime} 53^{\prime \prime} \mathrm{W}$;

samplingProtocol: Malaise intercept trap; verbatimEventDate: 20.iv.12; individualCount: 5; sex: male; lifeStage: adult; recordedBy: Rafael, J.A. | Limeira-de-Oliveira, F. | Takiya, D.M. | et al.; identifiedBy: Allan Paulo Moreira dos Santos; institutionCode: DZRJ; basisOfRecord: PreservedSpecimen

ab. country: Brazil; stateProvince: Ceará; municipality: Ubajara; locality: Parque Nacional de Ubajara, Trilha Araticum, Rio das Minas na altura da trilha do teleférico; maximumElevationInMeters: 420; verbatimCoordinates: $3^{\circ} 49^{\prime} 58^{\prime \prime S}, 40^{\circ} 53^{\prime} 53^{\prime \prime} \mathrm{W}$; samplingProtocol: Malaise intercept trap; verbatimEventDate: 20.iv.12; individualCount: 14; sex: female; lifeStage: adult; recordedBy: Rafael, J.A. | Limeira-de-Oliveira, F. | Takiya, D.M. | et al.; identifiedBy: Allan Paulo Moreira dos Santos; institutionCode: DZRJ; basisOfRecord: PreservedSpecimen

ac. country: Brazil; stateProvince: Ceará; municipality: Ubajara; locality: Parque Nacional de Ubajara, Rio Cafundó, pouco acima da cachoeira; maximumElevationInMeters: 795; verbatimCoordinates: $3^{\circ} 50^{\prime} 13^{\prime \prime S}, 40^{\circ} 54^{\prime} 35$ "W; samplingProtocol: Malaise intercept trap; verbatimEventDate: 21.iv.12; individualCount: 1; sex: male; lifeStage: adult; recordedBy: Rafael, J.A. | Limeira-de-Oliveira, F. | Takiya, D.M. | et al.; identifiedBy: Allan Paulo Moreira dos Santos; institutionCode: DZRJ; basisOfRecord: PreservedSpecimen

ad. country: Brazil; stateProvince: Ceará; municipality: Ubajara; locality: Parque Nacional de Ubajara, Trilha Araticum, Rio das Minas na altura da trilha do teleférico; maximumElevationInMeters: 420; verbatimCoordinates: $3^{\circ} 49^{\prime} 58^{\prime \prime} \mathrm{S}, 40^{\circ} 53^{\prime} 53^{\prime \prime} \mathrm{W}$; samplingProtocol: Malaise intercept trap; verbatimEventDate: 21.v.12; individualCount: 1; sex: male; lifeStage: adult; recordedBy: Limeira-de-Oliveira | et al.; identifiedBy: Allan Paulo Moreira dos Santos; institutionCode: DZRJ; basisOfRecord: PreservedSpecimen

ae. country: Brazil; stateProvince: Ceará; municipality: Ubajara; locality: Parque Nacional de Ubajara, Trilha Araticum, Rio das Minas na altura da trilha do teleférico; maximumElevationInMeters: 420; verbatimCoordinates: 349'58"S, 4053'53"W; samplingProtocol: Malaise intercept trap; verbatimEventDate: 21.v.12; individualCount: 3; sex: female; lifeStage: adult; recordedBy: Limeira-de-Oliveira | et al.; identifiedBy: Allan Paulo Moreira dos Santos; institutionCode: DZRJ; basisOfRecord: PreservedSpecimen

Distribution: Brazil!: CE!. Paraguay. Argentina.

Notes: New species record for Brazil.

\section{Family Ecnomidae}

Notes: New family record for CE.

\section{Genus Austrotinodes Schmid, 1955}

Notes: New genus record for CE.

\section{Austrotinodes paraguayensis* Flint, 1983}

\section{Materials}

a. country: Brazil; stateProvince: Ceará; municipality: Ubajara; locality: Parque Nacional de Ubajara, Rio Cafundó, pouco acima da cachoeira; maximumElevationInMeters: 795; verbatimCoordinates: $3^{\circ} 50^{\prime} 13^{\prime \prime S}, 40^{\circ} 54^{\prime} 35 " \mathrm{~W}$; sampling Protocol: Malaise intercept trap; 
verbatimEventDate: 1.ii.13; individualCount: 1; sex: male; lifeStage: adult; recordedBy: Limeira-de-Oliveira | et al.; identifiedBy: Wagner Rafael Maciel de Souza; institutionCode: DZRJ; basisOfRecord: PreservedSpecimen

b. $\quad$ country: Brazil; stateProvince: Ceará; municipality: Ubajara; locality: Parque Nacional de Ubajara, Trilha Samambaia, Mirante da cachoeira do Gameleira; maximumElevationInMeters: 880 ; verbatimCoordinates: $3^{\circ} 50^{\prime} 21$ 'S, 4054'23"W; samplingProtocol: Pennsylvania light trap; verbatimEventDate: 13.ii.13; individualCount: 1; sex: male; lifeStage: adult; recordedBy: Santos, A.P.M. | Takiya, D.M.; identifiedBy: Wagner Rafael Maciel de Souza; institutionCode: DZRJ; basisOfRecord: PreservedSpecimen

c. country: Brazil; stateProvince: Ceará; municipality: Ubajara; locality: Parque Nacional de Ubajara, Rio Cafundó, pouco acima da cachoeira; maximumElevationInMeters: 795; verbatimCoordinates: $3^{\circ} 50^{\prime} 13^{\prime \prime} \mathrm{S}, 40^{\circ} 54^{\prime} 35^{\prime \prime} \mathrm{W}$; samplingProtocol: Malaise intercept trap; verbatimEventDate: 13.xi.12; individualCount: 3; sex: male; lifeStage: adult; recordedBy: Limeira-de-Oliveira | et al.; identifiedBy: Wagner Rafael Maciel de Souza; institutionCode: DZRJ; basisOfRecord: PreservedSpecimen

d. country: Brazil; stateProvince: Ceará; municipality: Ubajara; locality: Parque Nacional de Ubajara, Trilha Samambaia, Rio Gameleira; maximumElevationInMeters: 874; verbatimCoordinates: $3^{\circ} 50^{\prime} 25 " \mathrm{~S}, 40^{\circ} 54^{\prime} 19^{\prime \prime} \mathrm{W}$; samplingProtocol: Pennsylvania light trap; verbatimEventDate: 14.ii.13; individualCount: 2; sex: female; lifeStage: adult; recordedBy: Santos, A.P.M. | Takiya, D.M.; identifiedBy: Wagner Rafael Maciel de Souza; institutionCode: DZRJ; basisOfRecord: PreservedSpecimen

e. $\quad$ country: Brazil; stateProvince: Ceará; municipality: Ubajara; locality: Parque Nacional de Ubajara, Trilha Araticum, Rio das Minas; maximumElevationInMeters: 524; verbatimCoordinates: $3^{\circ} 50^{\prime} 3$ "S, $40^{\circ} 54^{\prime} 18 \mathrm{~W}$; samplingProtocol: Malaise intercept trap; verbatimEventDate: 14.ii.13; individualCount: 4; sex: male; lifeStage: adult; recordedBy: Rafael, J.A. | Limeira-de-Oliveira, F. | Takiya, D.M. | Santos, A.P.M. | et al.; identifiedBy: Wagner Rafael Maciel de Souza; institutionCode: DZRJ; basisOfRecord: PreservedSpecimen

f. country: Brazil; stateProvince: Ceará; municipality: Ubajara; locality: Parque Nacional de Ubajara, Trilha Araticum, Rio das Minas; maximumElevationInMeters: 524; verbatimCoordinates: $3^{\circ} 50^{\prime} 3$ "S, $40^{\circ} 54^{\prime} 18^{\prime \prime W}$; samplingProtocol: Malaise intercept trap; verbatimEventDate: 14.ii.13; individualCount: 2; sex: female; lifeStage: adult; recordedBy: Rafael, J.A. | Limeira-de-Oliveira, F. | Takiya, D.M. | Santos, A.P.M. | et al.; identifiedBy: Wagner Rafael Maciel de Souza; institutionCode: DZRJ; basisOfRecord: PreservedSpecimen

g. country: Brazil; stateProvince: Ceará; municipality: Ubajara; locality: Parque Nacional de Ubajara, Cachoeira do Cafundó; maximumElevationInMeters: 783; verbatimCoordinates: $3^{\circ} 50^{\prime} 12^{\prime \prime} \mathrm{S}, 40^{\circ} 54^{\prime} 35 \mathrm{~W} \mathrm{~W}$; samplingProtocol: Pennsylvania light trap; verbatimEventDate: 15.ii.13; individualCount: 2; sex: male; lifeStage: adult; recordedBy: Santos, A.P.M. | Takiya, D.M.; identifiedBy: Wagner Rafael Maciel de Souza; institutionCode: DZRJ; basisOfRecord: PreservedSpecimen

h. country: Brazil; stateProvince: Ceará; municipality: Ubajara; locality: Parque Nacional de Ubajara, Trilha Samambaia, Rio Gameleira; maximumElevationInMeters: 874; verbatimCoordinates: $3^{\circ} 50^{\prime} 25 " \mathrm{~S}, 40^{\circ} 54^{\prime} 19 " \mathrm{~W}$; samplingProtocol: Pennsylvania light trap; verbatimEventDate: 18.iv.12; individualCount: 1; sex: female; lifeStage: adult; recordedBy: Takiya, D.M.; identifiedBy: Wagner Rafael Maciel de Souza; institutionCode: DZRJ; basisOfRecord: PreservedSpecimen

i. country: Brazil; stateProvince: Ceará; municipality: Ubajara; locality: Parque Nacional de Ubajara, Trilha Samambaia, Rio Gameleira; maximumElevationInMeters: 874; 
verbatimCoordinates: $3^{\circ} 50^{\prime 2} 25^{\prime \prime S}, 40^{\circ} 54^{\prime} 19^{\prime \prime} \mathrm{W}$; samplingProtocol: Malaise intercept trap; verbatimEventDate: 20.iv.12; individualCount: 1; sex: male; lifeStage: adult; recordedBy: Rafael, J.A. | Limeira-de-Oliveira, F. | Takiya, D.M. | et al.; identifiedBy: Wagner Rafael Maciel de Souza; institutionCode: DZRJ; basisOfRecord: PreservedSpecimen

j. country: Brazil; stateProvince: Ceará; municipality: Ubajara; locality: Parque Nacional de Ubajara, Trilha Samambaia, Rio Gameleira; maximumElevationInMeters: 874; verbatimCoordinates: $3^{\circ} 50^{\prime 2} 25^{\prime} \mathrm{S}, 40^{\circ} 54^{\prime} 19 " \mathrm{~W}$; samplingProtocol: Malaise intercept trap; verbatimEventDate: 20.iv.12; individualCount: 1; sex: female; lifeStage: adult; recordedBy: Rafael, J.A. | Limeira-de-Oliveira, F. | Takiya, D.M. | et al.; identifiedBy: Wagner Rafael Maciel de Souza; institutionCode: DZRJ; basisOfRecord: PreservedSpecimen

k. country: Brazil; stateProvince: Ceará; municipality: Ubajara; locality: Parque Nacional de Ubajara, Trilha Samambaia, Rio Gameleira; maximumElevationInMeters: 874; verbatimCoordinates: $3^{\circ} 50^{\prime} 25^{\prime \prime}$ S, 4054'19"W; samplingProtocol: Malaise intercept trap; verbatimEventDate: 20.iv.12; individualCount: 3; sex: male; lifeStage: adult; recordedBy: Rafael, J.A. | Limeira-de-Oliveira, F. | Takiya, D.M. | et al.; identifiedBy: Wagner Rafael Maciel de Souza; institutionCode: DZRJ; basisOfRecord: PreservedSpecimen

I. country: Brazil; stateProvince: Ceará; municipality: Ubajara; locality: Parque Nacional de Ubajara, Trilha Samambaia, Rio Gameleira; maximumElevationInMeters: 874; verbatimCoordinates: $3^{\circ} 50^{\prime} 25^{\prime \prime}$ S, $40^{\circ} 54^{\prime} 19^{\prime \prime W}$; samplingProtocol: Malaise intercept trap; verbatimEventDate: 20.iv.12; individualCount: 3; sex: female; lifeStage: adult; recordedBy: Rafael, J.A. | Limeira-de-Oliveira, F. | Takiya, D.M. | et al.; identifiedBy: Wagner Rafael Maciel de Souza; institutionCode: DZRJ; basisOfRecord: PreservedSpecimen

m. country: Brazil; stateProvince: Ceará; municipality: Ubajara; locality: Parque Nacional de Ubajara, Trilha Araticum, Rio das Minas na altura da trilha do teleférico; maximumElevationInMeters: 420; verbatimCoordinates: $3^{\circ} 49^{\prime} 58^{\prime \prime} \mathrm{S}, 40^{\circ} 53^{\prime} 53^{\prime \prime} \mathrm{W}$; samplingProtocol: Malaise intercept trap; verbatimEventDate: 20.iv.12; individualCount: 1; sex: male; lifeStage: adult; recordedBy: Rafael, J.A. | Limeira-de-Oliveira, F. | Takiya, D.M. | et al.; identifiedBy: Wagner Rafael Maciel de Souza; institutionCode: DZRJ; basisOfRecord: PreservedSpecimen

n. country: Brazil; stateProvince: Ceará; municipality: Ubajara; locality: Parque Nacional de Ubajara, Trilha Araticum, Rio das Minas na altura da trilha do teleférico; maximumElevationInMeters: 420; verbatimCoordinates: $3^{\circ} 49^{\prime} 58^{\prime \prime S}, 40^{\circ} 53^{\prime} 53^{\prime \prime} \mathrm{W}$; samplingProtocol: Malaise intercept trap; verbatimEventDate: 20.iv.12; individualCount: 1; sex: male; lifeStage: adult; recordedBy: Rafael, J.A. | Limeira-de-Oliveira, F. | Takiya, D.M. | et al.; identifiedBy: Wagner Rafael Maciel de Souza; institutionCode: DZRJ; basisOfRecord: PreservedSpecimen

o. country: Brazil; stateProvince: Ceará; municipality: Ubajara; locality: Parque Nacional de Ubajara, Trilha Araticum, Rio das Minas na altura da trilha do teleférico; maximumElevationInMeters: 420 ; verbatimCoordinates: $3^{\circ} 49^{\prime} 58^{\prime \prime S}, 40^{\circ} 53^{\prime} 53^{\prime \prime} \mathrm{W}$; samplingProtocol: Malaise intercept trap; verbatimEventDate: 20.iv.12; individualCount: 1; sex: male; lifeStage: adult; recordedBy: Rafael, J.A. | Limeira-de-Oliveira, F. | Takiya, D.M. | et al.; identifiedBy: Wagner Rafael Maciel de Souza; institutionCode: DZRJ; basisOfRecord: PreservedSpecimen

p. country: Brazil; stateProvince: Ceará; municipality: Ubajara; locality: Parque Nacional de Ubajara, Trilha Araticum, Rio das Minas na altura da trilha do teleférico; maximumElevationInMeters: 420; verbatimCoordinates: 349'58"S, 4053'53"W; samplingProtocol: Malaise intercept trap; verbatimEventDate: 20.iv.12; individualCount: 1; sex: female; lifeStage: adult; recordedBy: Rafael, J.A. | Limeira-de-Oliveira, F. | Takiya, D.M. | et al.; identifiedBy: Wagner Rafael Maciel de Souza; institutionCode: DZRJ; basisOfRecord: PreservedSpecimen 
q. country: Brazil; stateProvince: Ceará; municipality: Ubajara; locality: Parque Nacional de Ubajara, Trilha Araticum, Rio das Minas na altura da trilha do teleférico;

maximumElevationInMeters: 420; verbatimCoordinates: $3^{\circ} 49^{\prime} 58$ "S, 4053'53"W;

samplingProtocol: Malaise intercept trap; verbatimEventDate: 20.iv.12; individualCount: 1; sex: male; lifeStage: adult; recordedBy: Rafael, J.A. | Limeira-de-Oliveira, F. | Takiya, D.M. | et al.; identifiedBy: Wagner Rafael Maciel de Souza; institutionCode: DZRJ; basisOfRecord: PreservedSpecimen

r. country: Brazil; stateProvince: Ceará; municipality: Ubajara; locality: Parque Nacional de Ubajara, Trilha Araticum, Rio das Minas na altura da trilha do teleférico;

maximumElevationInMeters: 420; verbatimCoordinates: $3^{\circ} 49^{\prime} 58^{\prime \prime S}, 40^{\circ} 53^{\prime} 53 " \mathrm{~W}$;

samplingProtocol: Malaise intercept trap; verbatimEventDate: 20.iv.12; individualCount:

13; sex: male; lifeStage: adult; recordedBy: Rafael, J.A. | Limeira-de-Oliveira, F. | Takiya,

D.M. | et al.; identifiedBy: Wagner Rafael Maciel de Souza; institutionCode: DZRJ;

basisOfRecord: PreservedSpecimen

s. $\quad$ country: Brazil; stateProvince: Ceará; municipality: Ubajara; locality: Parque Nacional de Ubajara, Trilha Araticum, Rio das Minas na altura da trilha do teleférico;

maximumElevationInMeters: 420; verbatimCoordinates: $3^{\circ} 49^{\prime} 58^{\prime \prime S}, 40^{\circ} 53^{\prime} 53^{\prime \prime} \mathrm{W}$;

samplingProtocol: Malaise intercept trap; verbatimEventDate: 20.iv.12; individualCount:

12; sex: female; lifeStage: adult; recordedBy: Rafael, J.A. | Limeira-de-Oliveira, F. | Takiya,

D.M. | et al.; identifiedBy: Wagner Rafael Maciel de Souza; institutionCode: DZRJ;

basisOfRecord: PreservedSpecimen

t. country: Brazil; stateProvince: Ceará; municipality: Ubajara; locality: Parque Nacional de Ubajara, Trilha Araticum, Rio das Minas na altura da trilha do teleférico;

maximumElevationInMeters: 420; verbatimCoordinates: $3^{\circ} 49^{\prime} 58^{\prime \prime S}, 40^{\circ} 53^{\prime} 53$ "W;

samplingProtocol: Malaise intercept trap; verbatimEventDate: 20.iv.12; individualCount: 1; sex: female; lifeStage: adult; recordedBy: Rafael, J.A. | Limeira-de-Oliveira, F. | Takiya,

D.M. | et al.; identifiedBy: Wagner Rafael Maciel de Souza; institutionCode: DZRJ; basisOfRecord: PreservedSpecimen

u. country: Brazil; stateProvince: Ceará; municipality: Ubajara; locality: Parque Nacional de Ubajara, Trilha Samambaia, Rio Gameleira; maximumElevationInMeters: 874;

verbatimCoordinates: $3^{\circ} 50^{\prime} 25^{\prime \prime}$ S, $40^{\circ} 54^{\prime} 19 " \mathrm{~W}$; samplingProtocol: Pennsylvania light trap; verbatimEventDate: 21.iv.12; individualCount: 1; sex: male; lifeStage: adult; recordedBy:

Takiya, D.M. | Câmara, J.T.; identifiedBy: Wagner Rafael Maciel de Souza;

institutionCode: DZRJ; basisOfRecord: PreservedSpecimen

v. country: Brazil; stateProvince: Ceará; municipality: Ubajara; locality: Parque Nacional de Ubajara, Trilha Araticum, Rio das Minas na altura da trilha do teleférico;

maximumElevationInMeters: 420; verbatimCoordinates: $3^{\circ} 49^{\prime} 58^{\prime \prime} \mathrm{S}, 40^{\circ} 53^{\prime} 53^{\prime \prime} \mathrm{W}$;

samplingProtocol: Malaise intercept trap; verbatimEventDate: 21.v.12; individualCount: 6; sex: male; lifeStage: adult; recordedBy: Limeira-de-Oliveira | et al.; identifiedBy: Wagner Rafael Maciel de Souza; institutionCode: DZRJ; basisOfRecord: PreservedSpecimen

w. country: Brazil; stateProvince: Ceará; municipality: Ubajara; locality: Parque Nacional de Ubajara, Trilha Araticum, Rio das Minas na altura da trilha do teleférico;

maximumElevationInMeters: 420; verbatimCoordinates: $3^{\circ} 49^{\prime} 58^{\prime \prime S}, 40^{\circ} 53^{\prime} 53^{\prime \prime} \mathrm{W}$;

samplingProtocol: Malaise intercept trap; verbatimEventDate: 21.v.12; individualCount: 5; sex: female; lifeStage: adult; recordedBy: Limeira-de-Oliveira | et al.; identifiedBy: Wagner Rafael Maciel de Souza; institutionCode: DZRJ; basisOfRecord: PreservedSpecimen

x. country: Brazil; stateProvince: Ceará; municipality: Ubajara; locality: Parque Nacional de Ubajara, Trilha Samambaia, Mirante da cachoeira do Gameleira;

maximumElevationInMeters: 880; verbatimCoordinates: $3^{\circ} 50^{\prime 2} 21$ "S, 4054'23"W;

samplingProtocol: Pennsylvania light trap; verbatimEventDate: 23.iv.12; individualCount: 
1; sex: male; lifeStage: adult; recordedBy: Takiya, D.M. | Câmara, J.T.; identifiedBy: Wagner Rafael Maciel de Souza; institutionCode: DZRJ; basisOfRecord: PreservedSpecimen

Distribution: Brazil: PI!, CE!, MG. Paraguay.

Notes: New species record for Northeastern Brazil.

\section{Family Helicopsychidae}

\section{Genus Helicopsyche von Siebold, 1856}

\section{Helicopsyche (Feropsyche) monda Flint, 1983}

\section{Materials}

a. $\quad$ country: Brazil; stateProvince: Ceará; municipality: Ubajara; locality: Parque Nacional de Ubajara, Trilha Samambaia, Rio Gameleira; maximumElevationInMeters: 874; verbatimCoordinates: $3^{\circ} 50^{\prime 2} 25^{\prime} \mathrm{S}, 40^{\circ} 54^{\prime} 19^{\prime \prime} \mathrm{W}$; samplingProtocol: Pennsylvania light trap; verbatimEventDate: 13.ii.13; individualCount: 1; sex: male; lifeStage: adult; recordedBy: Santos, A.P.M. | Takiya, D.M.; identifiedBy: Allan Paulo Moreira dos Santos; institutionCode: DZRJ; basisOfRecord: PreservedSpecimen

b. $\quad$ country: Brazil; stateProvince: Ceará; municipality: Ubajara; locality: Parque Nacional de Ubajara, Trilha Samambaia, Rio Gameleira; maximumElevationInMeters: 874; verbatimCoordinates: $3^{\circ} 50^{\prime} 25 " \mathrm{~S}, 40^{\circ} 54^{\prime} 19^{\prime \prime} \mathrm{W}$; samplingProtocol: Pennsylvania light trap; verbatimEventDate: 13.ii.13; individualCount: 2; sex: female; lifeStage: adult; recordedBy: Santos, A.P.M. | Takiya, D.M.; identifiedBy: Allan Paulo Moreira dos Santos; institutionCode: DZRJ; basisOfRecord: PreservedSpecimen

c. country: Brazil; stateProvince: Ceará; municipality: Ubajara; locality: Parque Nacional de Ubajara, Trilha Samambaia, Rio Gameleira; maximumElevationInMeters: 874; verbatimCoordinates: $3^{\circ} 50^{\prime 2} 25^{\prime} \mathrm{S}, 40^{\circ} 54^{\prime} 19^{\prime \prime} \mathrm{W}$; samplingProtocol: Pennsylvania light trap; verbatimEventDate: 13.ii.13; individualCount: 4; sex: male; lifeStage: adult; recordedBy: Santos, A.P.M. | Takiya, D.M.; identifiedBy: Allan Paulo Moreira dos Santos; institutionCode: DZRJ; basisOfRecord: PreservedSpecimen

d. country: Brazil; stateProvince: Ceará; municipality: Ubajara; locality: Parque Nacional de Ubajara, Trilha Samambaia, Rio Gameleira; maximumElevationInMeters: 874; verbatimCoordinates: $3^{\circ} 50^{\prime} 25^{\prime \prime} \mathrm{S}, 40^{\circ} 54^{\prime} 19^{\prime \prime} \mathrm{W}$; samplingProtocol: Pennsylvania light trap; verbatimEventDate: 13.ii.13; individualCount: 1; sex: female; lifeStage: adult; recordedBy: Santos, A.P.M. | Takiya, D.M.; identifiedBy: Allan Paulo Moreira dos Santos; institutionCode: DZRJ; basisOfRecord: PreservedSpecimen

e. $\quad$ country: Brazil; stateProvince: Ceará; municipality: Ubajara; locality: Parque Nacional de Ubajara, Trilha Samambaia, Rio Gameleira; maximumElevationInMeters: 874; verbatimCoordinates: $3^{\circ} 50^{\prime} 25^{\prime \prime}$ S, $40^{\circ} 54^{\prime} 19 " \mathrm{~W}$; samplingProtocol: Pennsylvania light trap; verbatimEventDate: 14.ii.13; individualCount: 1; sex: male; lifeStage: adult; recordedBy: Santos, A.P.M. | Takiya, D.M.; identifiedBy: Allan Paulo Moreira dos Santos; institutionCode: DZRJ; basisOfRecord: PreservedSpecimen

f. country: Brazil; stateProvince: Ceará; municipality: Ubajara; locality: Parque Nacional de Ubajara, Trilha Araticum, Rio das Minas; maximumElevationInMeters: 524; verbatimCoordinates: $3^{\circ} 50^{\prime} 3$ "S, $40^{\circ} 54^{\prime} 18$ "W; samplingProtocol: Malaise intercept trap; 
verbatimEventDate: 14.ii.13; individualCount: 2; sex: male; lifeStage: adult; recordedBy: Rafael, J.A. | Limeira-de-Oliveira, F. | Takiya, D.M. | Santos, A.P.M. | et al.; identifiedBy: Allan Paulo Moreira dos Santos; institutionCode: DZRJ; basisOfRecord: PreservedSpecimen

g. country: Brazil; stateProvince: Ceará; municipality: Ubajara; locality: Parque Nacional de Ubajara, Cachoeira do Cafundó; maximumElevationInMeters: 783; verbatimCoordinates: 350'12"S, 4054'35"W; samplingProtocol: Pennsylvania light trap; verbatimEventDate: 15.ii.13; individualCount: 8; sex: male; lifeStage: adult; recordedBy: Santos, A.P.M. | Takiya, D.M.; identifiedBy: Allan Paulo Moreira dos Santos; institutionCode: DZRJ; basisOfRecord: PreservedSpecimen

h. $\quad$ country: Brazil; stateProvince: Ceará; municipality: Ubajara; locality: Parque Nacional de Ubajara, Ponte sobre Rio Miranda; maximumElevationInMeters: 792; verbatimCoordinates: $3^{\circ} 50^{\prime} 7.4^{\prime \prime S}, 40^{\circ} 54^{\prime} 47.5^{\prime \prime W}$; samplingProtocol: Pennsylvania light trap; verbatimEventDate: 15.ii.13; individualCount: 2; sex: male; lifeStage: adult; recordedBy: Santos, A.P.M. | Takiya, D.M.; identifiedBy: Allan Paulo Moreira dos Santos; institutionCode: DZRJ; basisOfRecord: PreservedSpecimen

i. country: Brazil; stateProvince: Ceará; municipality: Ubajara; locality: Parque Nacional de Ubajara, Ponte sobre Rio Miranda; maximumElevationInMeters: 792; verbatimCoordinates: $3^{\circ} 50^{\prime} 7.4^{\prime \prime S}, 40^{\circ} 54^{\prime} 47.5$ "W; samplingProtocol: Pennsylvania light trap; verbatimEventDate: 15.ii.13; individualCount: 2; sex: female; lifeStage: adult; recordedBy: Santos, A.P.M. | Takiya, D.M.; identifiedBy: Allan Paulo Moreira dos Santos; institutionCode: DZRJ; basisOfRecord: PreservedSpecimen

j. country: Brazil; stateProvince: Ceará; municipality: Ubajara; locality: Parque Nacional de Ubajara, Trilha Araticum, Rio das Minas na altura da trilha do teleférico; maximumElevationInMeters: 420; verbatimCoordinates: 349'58"S, 4053'53"W; samplingProtocol: Malaise intercept trap; verbatimEventDate: 20.iv.12; individualCount: 1; sex: male; lifeStage: adult; recordedBy: Rafael, J.A. | Limeira-de-Oliveira, F. | Takiya, D.M. I et al.; identifiedBy: Allan Paulo Moreira dos Santos; institutionCode: DZRJ; basisOfRecord: PreservedSpecimen

k. country: Brazil; stateProvince: Ceará; municipality: Ubajara; locality: Parque Nacional de Ubajara, Trilha Araticum, Rio das Minas na altura da trilha do teleférico; maximumElevationInMeters: 420; verbatimCoordinates: $3^{\circ} 49^{\prime} 58^{\prime \prime S}, 40^{\circ} 53^{\prime} 53^{\prime \prime} \mathrm{W}$; samplingProtocol: Malaise intercept trap; verbatimEventDate: 20.iv.12; individualCount: 1; sex: male; lifeStage: adult; recordedBy: Rafael, J.A. | Limeira-de-Oliveira, F. | Takiya, D.M. I et al.; identifiedBy: Allan Paulo Moreira dos Santos; institutionCode: DZRJ; basisOfRecord: PreservedSpecimen

I. country: Brazil; stateProvince: Ceará; municipality: Ubajara; locality: Parque Nacional de Ubajara, Trilha Samambaia, Rio Gameleira; maximumElevationInMeters: 874; verbatimCoordinates: $3^{\circ} 50^{\prime 2} 25^{\prime \prime}$, $40^{\circ} 54^{\prime} 19 " \mathrm{~W}$; samplingProtocol: Pennsylvania light trap; verbatimEventDate: 21.iv.12; individualCount: 2; sex: male; lifeStage: adult; recordedBy: Takiya, D.M. | Câmara, J.T.; identifiedBy: Allan Paulo Moreira dos Santos; institutionCode: DZRJ; basisOfRecord: PreservedSpecimen

m. country: Brazil; stateProvince: Ceará; municipality: Ubajara; locality: Parque Nacional de Ubajara, Trilha Samambaia, Rio Gameleira; maximumElevationInMeters: 874; verbatimCoordinates: $3^{\circ} 50^{\prime} 25 " \mathrm{~S}, 40^{\circ} 54^{\prime} 19 " \mathrm{~W}$; samplingProtocol: Pennsylvania light trap; verbatimEventDate: 21.iv.12; individualCount: 1; sex: female; lifeStage: adult; recordedBy: Takiya, D.M. | Câmara, J.T.; identifiedBy: Allan Paulo Moreira dos Santos; institutionCode: DZRJ; basisOfRecord: PreservedSpecimen

n. country: Brazil; stateProvince: Ceará; municipality: Ubajara; locality: Parque Nacional de Ubajara, Trilha Samambaia, Rio Gameleira; maximumElevationInMeters: 874; 
verbatimCoordinates: $3^{\circ} 50^{\prime} 25^{\prime \prime}$ S, $40^{\circ} 54^{\prime} 19^{\prime \prime} \mathrm{W}$; samplingProtocol: Pennsylvania light trap; verbatimEventDate: 21.iv.12; individualCount: 4; sex: male; lifeStage: adult; recordedBy: Takiya, D.M. | Câmara, J.T.; identifiedBy: Allan Paulo Moreira dos Santos; institutionCode: DZRJ; basisOfRecord: PreservedSpecimen

o. country: Brazil; stateProvince: Ceará; municipality: Ubajara; locality: Parque Nacional de Ubajara, Rio Cafundó, pouco acima da cachoeira; maximumElevationInMeters: 795; verbatimCoordinates: $3^{\circ} 50^{\prime} 13^{\prime \prime} \mathrm{S}, 40^{\circ} 54^{\prime} 35 \mathrm{~W} \mathrm{~W}$; samplingProtocol: Malaise intercept trap; verbatimEventDate: 21.iv.12; individualCount: 3; sex: male; lifeStage: adult; recordedBy: Rafael, J.A. | Limeira-de-Oliveira, F. | Takiya, D.M. | et al.; identifiedBy: Allan Paulo Moreira dos Santos; institutionCode: DZRJ; basisOfRecord: PreservedSpecimen

p. country: Brazil; stateProvince: Ceará; municipality: Ubajara; locality: Parque Nacional de Ubajara, Rio Cafundó, pouco acima da cachoeira; maximumElevationInMeters: 795; verbatimCoordinates: $3^{\circ} 50^{\prime} 13^{\prime \prime} \mathrm{S}, 40^{\circ} 54^{\prime} 35 \mathrm{WW}$; samplingProtocol: Malaise intercept trap; verbatimEventDate: 21.iv.12; individualCount: 1; sex: female; lifeStage: adult; recordedBy: Rafael, J.A. | Limeira-de-Oliveira, F. | Takiya, D.M. | et al.; identifiedBy: Allan Paulo Moreira dos Santos; institutionCode: DZRJ; basisOfRecord: PreservedSpecimen

q. country: Brazil; stateProvince: Ceará; municipality: Ubajara; locality: Parque Nacional de Ubajara, Trilha Samambaia, Mirante da cachoeira do Gameleira; maximumElevationInMeters: 880; verbatimCoordinates: $3^{\circ} 50^{\prime} 21$ 'S, 4054'23"W; samplingProtocol: Pennsylvania light trap; verbatimEventDate: 23.iv.12; individualCount: 4; sex: male; lifeStage: adult; recordedBy: Takiya, D.M. | Câmara, J.T.; identifiedBy: Allan Paulo Moreira dos Santos; institutionCode: DZRJ; basisOfRecord: PreservedSpecimen

r. country: Brazil; stateProvince: Ceará; municipality: Ubajara; locality: Parque Nacional de Ubajara, Trilha Samambaia, Mirante da cachoeira do Gameleira; maximumElevationInMeters: 880; verbatimCoordinates: $3^{\circ} 50^{\prime} 21^{\prime \prime S}, 40^{\circ} 54^{\prime} 23^{\prime \prime W}$; sampling Protocol: Pennsylvania light trap; verbatimEventDate: 23.iv.12; individualCount: 8; sex: female; lifeStage: adult; recordedBy: Takiya, D.M. | Câmara, J.T.; identifiedBy: Allan Paulo Moreira dos Santos; institutionCode: DZRJ; basisOfRecord: PreservedSpecimen

s. $\quad$ country: Brazil; stateProvince: Ceará; municipality: Ubajara; locality: Parque Nacional de Ubajara, Rio Cafundó, pouco acima da cachoeira; maximumElevationInMeters: 795; verbatimCoordinates: $3^{\circ} 50^{\prime} 13^{\prime \prime} \mathrm{S}, 40^{\circ} 54^{\prime} 35 " \mathrm{~W}$; samplingProtocol: Pennsylvania light trap; verbatimEventDate: 24.iv.12; individualCount: 3; sex: male; lifeStage: adult; recordedBy: Takiya, D.M. | Rafael, J.A.; identifiedBy: Allan Paulo Moreira dos Santos; institutionCode: DZRJ; basisOfRecord: PreservedSpecimen

Distribution: Brazil: CE!, MG, SP, SC.

Notes: New species record for Northeastern Brazil.

\section{Helicopsyche (Feropsyche) vergelana* Ross, 1956}

\section{Materials}

a. country: Brazil; stateProvince: Ceará; municipality: Ubajara; locality: Parque Nacional de Ubajara, Rio Cafundó, pouco acima da cachoeira; maximumElevationInMeters: 795; verbatimCoordinates: $3^{\circ} 50^{\prime} 13^{\prime \prime} \mathrm{S}, 40^{\circ} 54^{\prime} 35 \mathrm{WW}$; samplingProtocol: Malaise intercept trap; verbatimEventDate: 13.ix.12; individualCount: 2; sex: male; lifeStage: adult; recordedBy: Limeira-de-Oliveira | et al.; identifiedBy: Allan Paulo Moreira dos Santos; institutionCode: DZRJ; basisOfRecord: PreservedSpecimen 
b. country: Brazil; stateProvince: Ceará; municipality: Ubajara; locality: Parque Nacional de Ubajara, Rio Cafundó, pouco acima da cachoeira; maximumElevationInMeters: 795; verbatimCoordinates: $3^{\circ} 50^{\prime} 13^{\prime \prime} \mathrm{S}, 40^{\circ} 54^{\prime} 35 \mathrm{WW}$; samplingProtocol: Malaise intercept trap; verbatimEventDate: 13.xi.12; individualCount: 1; sex: male; lifeStage: adult; recordedBy: Limeira-de-Oliveira | et al.; identifiedBy: Allan Paulo Moreira dos Santos; institutionCode: DZRJ; basisOfRecord: PreservedSpecimen

c. country: Brazil; stateProvince: Ceará; municipality: Ubajara; locality: Parque Nacional de Ubajara, Rio Cafundó, pouco acima da cachoeira; maximumElevationInMeters: 795; verbatimCoordinates: $3^{\circ} 50^{\prime} 13^{\prime \prime} \mathrm{S}, 40^{\circ} 54^{\prime} 35^{\prime \prime} \mathrm{W}$; samplingProtocol: Malaise intercept trap; verbatimEventDate: 13.xi.12; individualCount: 4; sex: female; lifeStage: adult; recordedBy: Limeira-de-Oliveira | et al.; identifiedBy: Allan Paulo Moreira dos Santos; institutionCode: DZRJ; basisOfRecord: PreservedSpecimen

d. country: Brazil; stateProvince: Ceará; municipality: Ubajara; locality: Parque Nacional de Ubajara, Trilha Araticum, Rio das Minas; maximumElevationInMeters: 524;

verbatimCoordinates: $3^{\circ} 50 ' 3 " \mathrm{~S}, 40^{\circ} 54^{\prime} 18 \mathrm{~W}$; samplingProtocol: Malaise intercept trap; verbatimEventDate: 14.ii.13; individualCount: 32; sex: male; lifeStage: adult; recordedBy: Rafael, J.A. | Limeira-de-Oliveira, F. | Takiya, D.M. | Santos, A.P.M. | et al.; identifiedBy: Allan Paulo Moreira dos Santos; institutionCode: DZRJ; basisOfRecord: PreservedSpecimen

e. country: Brazil; stateProvince: Ceará; municipality: Ubajara; locality: Parque Nacional de Ubajara, Trilha Araticum, Rio das Minas na altura da trilha do teleférico; maximumElevationInMeters: 420; verbatimCoordinates: $3^{\circ} 49^{\prime} 58^{\prime \prime S}, 40^{\circ} 53^{\prime} 53^{\prime \prime} \mathrm{W}$; samplingProtocol: Malaise intercept trap; verbatimEventDate: 14.ii.13; individualCount: 4; sex: male; lifeStage: adult; recordedBy: Rafael, J.A. | Limeira-de-Oliveira, F. | Takiya, D.M. | Santos, A.P.M. | et al.; identifiedBy: Allan Paulo Moreira dos Santos; institutionCode: DZRJ; basisOfRecord: PreservedSpecimen

f. country: Brazil; stateProvince: Ceará; municipality: Ubajara; locality: Parque Nacional de Ubajara, Trilha Araticum, Rio das Minas na altura da trilha do teleférico; maximumElevationInMeters: 420; verbatimCoordinates: 349'58"S, 4053'53"W; samplingProtocol: Malaise intercept trap; verbatimEventDate: 14.ii.13; individualCount: 36; sex: female; lifeStage: adult; recordedBy: Rafael, J.A. | Limeira-de-Oliveira, F. | Takiya, D.M. | Santos, A.P.M. | et al.; identifiedBy: Allan Paulo Moreira dos Santos; institutionCode: DZRJ; basisOfRecord: PreservedSpecimen

g. country: Brazil; stateProvince: Ceará; municipality: Ubajara; locality: Parque Nacional de Ubajara, Trilha Araticum, Rio da Minas abaixo do teleférico; maximumElevationInMeters: 395; verbatimCoordinates: $3^{\circ} 49^{\prime} 43.3$ "S, 4053'51.5"W; samplingProtocol: Pennsylvania light trap; verbatimEventDate: 14.ii.13; individualCount: 1; sex: male; lifeStage: adult; recordedBy: Santos, A.P.M. | Takiya, D.M.; identifiedBy: Allan Paulo Moreira dos Santos; institutionCode: DZRJ; basisOfRecord: PreservedSpecimen

h. country: Brazil; stateProvince: Ceará; municipality: Ubajara; locality: Parque Nacional de Ubajara, Cachoeira do Cafundó; maximumElevationInMeters: 783; verbatimCoordinates: $3^{\circ} 50^{\prime} 12 " \mathrm{~S}, 40^{\circ} 54^{\prime} 35^{\prime \prime} \mathrm{W}$; samplingProtocol: Pennsylvania light trap; verbatimEventDate: 15.ii.13; individualCount: 2; sex: male; lifeStage: adult; recordedBy: Santos, A.P.M. | Takiya, D.M.; identifiedBy: Allan Paulo Moreira dos Santos; institutionCode: DZRJ; basisOfRecord: PreservedSpecimen

i. country: Brazil; stateProvince: Ceará; municipality: Ubajara; locality: Parque Nacional de Ubajara, Trilha Araticum, Rio das Minas; maximumElevationInMeters: 524; verbatimCoordinates: $3^{\circ} 50^{\prime} 3$ "S, $40^{\circ} 54^{\prime} 18 \mathrm{WW}$; samplingProtocol: Malaise intercept trap; verbatimEventDate: 17.ii.13; individualCount: 4; sex: male; lifeStage: adult; recordedBy: Rafael, J.A. | Limeira-de-Oliveira, F. | Takiya, D.M. | Santos, A.P.M. | et al.; identifiedBy: 
Allan Paulo Moreira dos Santos; institutionCode: DZRJ; basisOfRecord: PreservedSpecimen

j. $\quad$ country: Brazil; stateProvince: Ceará; municipality: Ubajara; locality: Parque Nacional de Ubajara, Trilha Araticum, Rio das Minas; maximumElevationInMeters: 524; verbatimCoordinates: $3^{\circ} 50^{\prime} 3$ "S, $40^{\circ} 54^{\prime} 18^{\prime \prime} \mathrm{W}$; samplingProtocol: Malaise intercept trap; verbatimEventDate: 17.ii.13; individualCount: 4; sex: female; lifeStage: adult; recordedBy: Rafael, J.A. | Limeira-de-Oliveira, F. | Takiya, D.M. | Santos, A.P.M. | et al.; identifiedBy: Allan Paulo Moreira dos Santos; institutionCode: DZRJ; basisOfRecord: PreservedSpecimen

k. country: Brazil; stateProvince: Ceará; municipality: Ubajara; locality: Parque Nacional de Ubajara, Trilha Araticum, Rio das Minas na altura da trilha do teleférico; maximumElevationInMeters: 420; verbatimCoordinates: $3^{\circ} 49^{\prime} 58^{\prime \prime S}, 40^{\circ} 53^{\prime} 53^{\prime \prime} \mathrm{W}$; samplingProtocol: Malaise intercept trap; verbatimEventDate: 20.iv.12; individualCount: 1; sex: male; lifeStage: adult; recordedBy: Rafael, J.A. | Limeira-de-Oliveira, F. | Takiya, D.M. | et al.; identifiedBy: Allan Paulo Moreira dos Santos; institutionCode: DZRJ; basisOfRecord: PreservedSpecimen

I. country: Brazil; stateProvince: Ceará; municipality: Ubajara; locality: Parque Nacional de Ubajara, Trilha Araticum, Rio das Minas na altura da trilha do teleférico; maximumElevationInMeters: 420; verbatimCoordinates: 349'58"S, 4053'53"W; samplingProtocol: Malaise intercept trap; verbatimEventDate: 20.iv.12; individualCount: 1; sex: male; lifeStage: adult; recordedBy: Rafael, J.A. | Limeira-de-Oliveira, F. | Takiya, D.M. | et al.; identifiedBy: Allan Paulo Moreira dos Santos; institutionCode: DZRJ; basisOfRecord: PreservedSpecimen

m. country: Brazil; stateProvince: Ceará; municipality: Ubajara; locality: Parque Nacional de Ubajara, Trilha Araticum, Rio das Minas na altura da trilha do teleférico; maximumElevationInMeters: 420; verbatimCoordinates: $3^{\circ} 49^{\prime} 58^{\prime \prime S}, 40^{\circ} 53^{\prime} 53^{\prime \prime W}$; samplingProtocol: Malaise intercept trap; verbatimEventDate: 20.iv.12; individualCount: 2; sex: female; lifeStage: adult; recordedBy: Rafael, J.A. | Limeira-de-Oliveira, F. | Takiya, D.M. | et al.; identifiedBy: Allan Paulo Moreira dos Santos; institutionCode: DZRJ; basisOfRecord: PreservedSpecimen

n. country: Brazil; stateProvince: Ceará; municipality: Ubajara; locality: Parque Nacional de Ubajara, Trilha Araticum, Rio das Minas na altura da trilha do teleférico; maximumElevationInMeters: 420; verbatimCoordinates: $3^{\circ} 49^{\prime} 58$ "S, 4053'53"W; samplingProtocol: Malaise intercept trap; verbatimEventDate: 20.iv.12; individualCount: 3; sex: male; lifeStage: adult; recordedBy: Rafael, J.A. | Limeira-de-Oliveira, F. | Takiya, D.M. | et al.; identifiedBy: Allan Paulo Moreira dos Santos; institutionCode: DZRJ; basisOfRecord: PreservedSpecimen

o. country: Brazil; stateProvince: Ceará; municipality: Ubajara; locality: Parque Nacional de Ubajara, Trilha Araticum, Rio das Minas na altura da trilha do teleférico; maximumElevationInMeters: 420; verbatimCoordinates: 349'58"S, 4053'53"W; samplingProtocol: Malaise intercept trap; verbatimEventDate: 20.iv.12; individualCount: 6; sex: male; lifeStage: adult; recordedBy: Rafael, J.A. | Limeira-de-Oliveira, F. | Takiya, D.M. | et al.; identifiedBy: Allan Paulo Moreira dos Santos; institutionCode: DZRJ; basisOfRecord: PreservedSpecimen

p. country: Brazil; stateProvince: Ceará; municipality: Ubajara; locality: Parque Nacional de Ubajara, Trilha Araticum, Rio das Minas na altura da trilha do teleférico; maximumElevationInMeters: 420; verbatimCoordinates: $3^{\circ} 49^{\prime} 58^{\prime \prime S}, 40^{\circ} 53^{\prime} 53^{\prime \prime} \mathrm{W}$; samplingProtocol: Malaise intercept trap; verbatimEventDate: 20.iv.12; individualCount: 11; sex: female; lifeStage: adult; recordedBy: Rafael, J.A. | Limeira-de-Oliveira, F. | Takiya, 
D.M. | et al.; identifiedBy: Allan Paulo Moreira dos Santos; institutionCode: DZRJ; basisOfRecord: PreservedSpecimen

q. country: Brazil; stateProvince: Ceará; municipality: Ubajara; locality: Parque Nacional de Ubajara, Trilha Araticum, Rio das Minas na altura da trilha do teleférico; maximumElevationInMeters: 420; verbatimCoordinates: $3^{\circ} 49^{\prime} 58^{\prime \prime S}, 40^{\circ} 53^{\prime} 53^{\prime \prime} \mathrm{W}$; samplingProtocol: Malaise intercept trap; verbatimEventDate: 20.iv.12; individualCount: 20; sex: male; lifeStage: adult; recordedBy: Rafael, J.A. | Limeira-de-Oliveira, F. | Takiya, D.M. | et al.; identifiedBy: Allan Paulo Moreira dos Santos; institutionCode: DZRJ; basisOfRecord: PreservedSpecimen

r. country: Brazil; stateProvince: Ceará; municipality: Ubajara; locality: Parque Nacional de Ubajara, Rio Cafundó, pouco acima da cachoeira; maximumElevationInMeters: 795; verbatimCoordinates: $3^{\circ} 50^{\prime} 13^{\prime \prime} \mathrm{S}, 40^{\circ} 54^{\prime} 35 \mathrm{WW}$; samplingProtocol: Malaise intercept trap; verbatimEventDate: 21.iv.12; individualCount: 15; sex: male; lifeStage: adult; recordedBy: Rafael, J.A. | Limeira-de-Oliveira, F. | Takiya, D.M. | et al.; identifiedBy: Allan Paulo Moreira dos Santos; institutionCode: DZRJ; basisOfRecord: PreservedSpecimen

s. $\quad$ country: Brazil; stateProvince: Ceará; municipality: Ubajara; locality: Parque Nacional de Ubajara, Trilha Araticum, Rio das Minas na altura da trilha do teleférico; maximumElevationInMeters: 420; verbatimCoordinates: $3^{\circ} 49^{\prime} 58^{\prime \prime} \mathrm{S}, 40^{\circ} 53^{\prime} 53^{\prime \prime} \mathrm{W}$; samplingProtocol: Malaise intercept trap; verbatimEventDate: 21.v.12; individualCount: 18; sex: male; lifeStage: adult; recordedBy: Limeira-de-Oliveira | et al.; identifiedBy: Allan Paulo Moreira dos Santos; institutionCode: DZRJ; basisOfRecord: PreservedSpecimen

t. country: Brazil; stateProvince: Ceará; municipality: Ubajara; locality: Parque Nacional de Ubajara, Trilha Araticum, Rio das Minas na altura da trilha do teleférico; maximumElevationInMeters: 420; verbatimCoordinates: 349'58"S, 4053'53"W; samplingProtocol: Malaise intercept trap; verbatimEventDate: 21.v.12; individualCount: 38; sex: female; lifeStage: adult; recordedBy: Limeira-de-Oliveira | et al.; identifiedBy: Allan Paulo Moreira dos Santos; institutionCode: DZRJ; basisOfRecord: PreservedSpecimen

u. $\quad$ country: Brazil; stateProvince: Ceará; municipality: Ubajara; locality: Parque Nacional de Ubajara, Trilha Araticum, Rio das Minas; maximumElevationInMeters: 524; verbatimCoordinates: $3^{\circ} 50^{\prime} 3 " \mathrm{~S}, 40^{\circ} 54^{\prime} 18 \mathrm{~W}$; samplingProtocol: Pennsylvania light trap; verbatimEventDate: 22.iv.12; individualCount: 2; sex: male; lifeStage: adult; recordedBy: Takiya, D.M. | Somavilla, A.; identifiedBy: Allan Paulo Moreira dos Santos; institutionCode: DZRJ; basisOfRecord: PreservedSpecimen

v. country: Brazil; stateProvince: Ceará; municipality: Ubajara; locality: Parque Nacional de Ubajara, Trilha Araticum, Rio das Minas; maximumElevationInMeters: 524; verbatimCoordinates: $3^{\circ} 50^{\prime} 3$ "S, $40^{\circ} 54^{\prime} 18$ "W; sampling Protocol: Pennsylvania light trap; verbatimEventDate: 22.iv.12; individualCount: 18; sex: female; lifeStage: adult; recordedBy: Takiya, D.M. | Somavilla, A.; identifiedBy: Allan Paulo Moreira dos Santos; institutionCode: DZRJ; basisOfRecord: PreservedSpecimen

w. country: Brazil; stateProvince: Ceará; municipality: Ubajara; locality: Parque Nacional de Ubajara, Rio Cafundó, pouco acima da cachoeira; maximumElevationInMeters: 795; verbatimCoordinates: $3^{\circ} 50^{\prime} 13^{\prime \prime}$ S, $40^{\circ} 54^{\prime} 35 " \mathrm{~W}$; samplingProtocol: Pennsylvania light trap; verbatimEventDate: 24.iv.12; individualCount: 1; sex: male; lifeStage: adult; recordedBy: Takiya, D.M. | Rafael, J.A.; identifiedBy: Allan Paulo Moreira dos Santos; institutionCode: DZRJ; basisOfRecord: PreservedSpecimen

Distribution: Mexico. Belize. Guatemala. Honduras. Costa Rica. Panama. Trinidad and Tobago. Venezuela. Suriname. Brazil: PI!, CE!, PE. Peru. 
Notes: New species record for CE.

\section{Family Hydropsychidae}

\section{Genus Leptonema Guérin, 1843}

\section{Leptonema spinulum Flint, McAlpine \& Ross, 1987}

\section{Material}

a. country: Brazil; stateProvince: Ceará; municipality: Ubajara; locality: Parque Nacional de Ubajara, Rio Cafundó, pouco acima da cachoeira; maximumElevationInMeters: 795; verbatimCoordinates: $3^{\circ} 50^{\prime} 13^{\prime \prime} \mathrm{S}, 40^{\circ} 54^{\prime} 35 " \mathrm{~W}$; samplingProtocol: Pennsylvania light trap; verbatimEventDate: 24.iv.12; individualCount: 1; sex: male; lifeStage: adult; recordedBy: Takiya, D.M. | Rafael, J.A.; identifiedBy: Allan Paulo Moreira dos Santos; institutionCode: DZRJ; basisOfRecord: PreservedSpecimen

Distribution: Venezuela. Guyana. Brazil: PA, AM, CE!, MT, DF. Peru. Argentina.

Notes: New species record for Northeastern Brazil.

\section{Leptonema viridianum* Navás, 1916}

\section{Materials}

a. $\quad$ country: Brazil; stateProvince: Ceará; municipality: Ubajara; locality: Parque Nacional de Ubajara, Rio Cafundó, pouco acima da cachoeira; maximumElevationInMeters: 795; verbatimCoordinates: $3^{\circ} 50^{\prime} 13^{\prime \prime S}$, 4054'35"W; samplingProtocol: Malaise intercept trap; verbatimEventDate: 1.ii.13; individualCount: 1; sex: female; lifeStage: adult; recordedBy: Limeira-de-Oliveira | et al.; identifiedBy: Allan Paulo Moreira dos Santos; institutionCode: DZRJ; basisOfRecord: PreservedSpecimen

b. country: Brazil; stateProvince: Ceará; municipality: Ubajara; locality: Parque Nacional de Ubajara, Rio Cafundó, pouco acima da cachoeira; maximumElevationInMeters: 795; verbatimCoordinates: $3^{\circ} 50^{\prime} 13^{\prime \prime S}, 40^{\circ} 54^{\prime} 35 " \mathrm{~W}$; samplingProtocol: Malaise intercept trap; verbatimEventDate: 13.xi.12; individualCount: 1; sex: male; lifeStage: adult; recordedBy: Limeira-de-Oliveira | et al.; identifiedBy: Allan Paulo Moreira dos Santos; institutionCode: DZRJ; basisOfRecord: PreservedSpecimen

c. country: Brazil; stateProvince: Ceará; municipality: Ubajara; locality: Parque Nacional de Ubajara, Trilha Araticum, Rio das Minas; maximumElevationInMeters: 524; verbatimCoordinates: $3^{\circ} 50^{\prime} 3 " \mathrm{~S}, 40^{\circ} 54^{\prime} 18 \mathrm{~W}$; samplingProtocol: Pennsylvania light trap; verbatimEventDate: 14.ii.13; individualCount: 1; sex: female; lifeStage: adult; recordedBy: Santos, A.P.M. | Takiya, D.M.; identifiedBy: Allan Paulo Moreira dos Santos; institutionCode: DZRJ; basisOfRecord: PreservedSpecimen

d. country: Brazil; stateProvince: Ceará; municipality: Ubajara; locality: Parque Nacional de Ubajara, Trilha Araticum, Rio das Minas; maximumElevationInMeters: 524; verbatimCoordinates: $3^{\circ} 50 ' 3 " \mathrm{~S}, 40^{\circ} 54^{\prime} 18 \mathrm{~W}$; samplingProtocol: Pennsylvania light trap; verbatimEventDate: 14.ii.13; individualCount: 1; sex: male; lifeStage: adult; recordedBy: Santos, A.P.M. | Takiya, D.M.; identifiedBy: Allan Paulo Moreira dos Santos; institutionCode: DZRJ; basisOfRecord: PreservedSpecimen 
e. $\quad$ country: Brazil; stateProvince: Ceará; municipality: Ubajara; locality: Parque Nacional de Ubajara, Trilha Araticum, Rio das Minas; maximumElevationInMeters: 524; verbatimCoordinates: $3^{\circ} 50^{\prime} 3$ "S, $40^{\circ} 54^{\prime} 18 \mathrm{~W} \mathrm{~W}$; samplingProtocol: Pennsylvania light trap; verbatimEventDate: 14.ii.13; individualCount: 1; sex: female; lifeStage: adult; recordedBy: Santos, A.P.M. | Takiya, D.M.; identifiedBy: Allan Paulo Moreira dos Santos; institutionCode: DZRJ; basisOfRecord: PreservedSpecimen

f. country: Brazil; stateProvince: Ceará; municipality: Ubajara; locality: Parque Nacional de Ubajara, Trilha Araticum, Rio das Minas; maximumElevationInMeters: 524; verbatimCoordinates: $3^{\circ} 50^{\prime} 3$ "S, $40^{\circ} 54^{\prime} 18 \mathrm{~W}$; samplingProtocol: Pennsylvania light trap; verbatimEventDate: 14.ii.13; individualCount: 5; sex: male; lifeStage: adult; recordedBy: Santos, A.P.M. | Takiya, D.M.; identifiedBy: Allan Paulo Moreira dos Santos; institutionCode: DZRJ; basisOfRecord: PreservedSpecimen

g. country: Brazil; stateProvince: Ceará; municipality: Ubajara; locality: Parque Nacional de Ubajara, Trilha Araticum, Rio das Minas na altura da trilha do teleférico; maximumElevationInMeters: 420; verbatimCoordinates: $3^{\circ} 49^{\prime} 58$ "S, 4053'53"W; samplingProtocol: Malaise intercept trap; verbatimEventDate: 14.ii.13; individualCount: 3; sex: male; lifeStage: adult; recordedBy: Rafael, J.A. | Limeira-de-Oliveira, F. | Takiya, D.M. | Santos, A.P.M. | et al.; identifiedBy: Allan Paulo Moreira dos Santos; institutionCode: DZRJ; basisOfRecord: PreservedSpecimen

h. country: Brazil; stateProvince: Ceará; municipality: Ubajara; locality: Parque Nacional de Ubajara, Rio das Minas, próximo ao Portăo Araticum; maximumElevationInMeters: 328; verbatimCoordinates: $3^{\circ} 49^{\prime} 32.6^{\prime \prime} \mathrm{S}, 40^{\circ} 53^{\prime} 32.8^{\prime \prime} \mathrm{W}$; samplingProtocol: Malaise intercept trap; verbatimEventDate: 14.ii.13; individualCount: 6; sex: female; lifeStage: adult; recordedBy: Rafael, J.A. | Limeira-de-Oliveira, F. | Takiya, D.M. | Santos, A.P.M. | et al.; identifiedBy: Allan Paulo Moreira dos Santos; institutionCode: DZRJ; basisOfRecord: PreservedSpecimen

i. country: Brazil; stateProvince: Ceará; municipality: Ubajara; locality: Parque Nacional de Ubajara, Trilha Araticum, Rio das Minas; maximumElevationInMeters: 524; verbatimCoordinates: $3^{\circ} 50^{\prime} 3^{\prime \prime} \mathrm{S}, 40^{\circ} 54^{\prime} 18^{\prime \prime} \mathrm{W}$; samplingProtocol: Manual; verbatimEventDate: 17.ii.13; individualCount: 3; sex: male; lifeStage: adult; recordedBy: Santos, A.P.M. | Takiya, D.M.; identifiedBy: Allan Paulo Moreira dos Santos; institutionCode: DZRJ; basisOfRecord: PreservedSpecimen

j. $\quad$ country: Brazil; stateProvince: Ceará; municipality: Ubajara; locality: Parque Nacional de Ubajara, Rio das Minas, próximo ao Portăo Araticum; maximumElevationInMeters: 328; verbatimCoordinates: $3^{\circ} 49^{\prime} 32.6$ "S, 4053'32.8"W; samplingProtocol: Malaise intercept trap; verbatimEventDate: 17.ii.13; individualCount: 3; sex: female; lifeStage: adult; recordedBy: Rafael, J.A. | Limeira-de-Oliveira, F. | Takiya, D.M. | Santos, A.P.M. | et al.; identifiedBy: Allan Paulo Moreira dos Santos; institutionCode: DZRJ; basisOfRecord: PreservedSpecimen

k. country: Brazil; stateProvince: Ceará; municipality: Ubajara; locality: Parque Nacional de Ubajara, Trilha Samambaia, Rio Gameleira; maximumElevationInMeters: 874; verbatimCoordinates: $3^{\circ} 50^{\prime} 25^{\prime \prime}$ S, $40^{\circ} 54^{\prime} 19^{\prime \prime} \mathrm{W}$; samplingProtocol: Malaise intercept trap; verbatimEventDate: 20.iv.12; individualCount: 2; sex: female; lifeStage: adult; recordedBy: Rafael, J.A. | Limeira-de-Oliveira, F. | Takiya, D.M. | et al.; identifiedBy: Allan Paulo Moreira dos Santos; institutionCode: DZRJ; basisOfRecord: PreservedSpecimen

I. country: Brazil; stateProvince: Ceará; municipality: Ubajara; locality: Parque Nacional de Ubajara, Trilha Araticum, Rio das Minas na altura da trilha do teleférico; maximumElevationInMeters: 420 ; verbatimCoordinates: $3^{\circ} 49^{\prime} 58^{\prime \prime S}, 40^{\circ} 53^{\prime} 53^{\prime \prime} \mathrm{W}$; samplingProtocol: Malaise intercept trap; verbatimEventDate: $20 . i v .12$; individualCount: 10; sex: male; lifeStage: adult; recordedBy: Rafael, J.A. | Limeira-de-Oliveira, F. | Takiya, 
D.M. | et al.; identifiedBy: Allan Paulo Moreira dos Santos; institutionCode: DZRJ; basisOfRecord: PreservedSpecimen

m. country: Brazil; stateProvince: Ceará; municipality: Ubajara; locality: Parque Nacional de Ubajara, Trilha Araticum, Rio das Minas na altura da trilha do teleférico; maximumElevationInMeters: 420; verbatimCoordinates: $3^{\circ} 49^{\prime} 58^{\prime \prime} \mathrm{S}, 40^{\circ} 53^{\prime} 53^{\prime \prime} \mathrm{W}$; samplingProtocol: Malaise intercept trap; verbatimEventDate: 20.iv.12; individualCount: 57; sex: female; lifeStage: adult; recordedBy: Rafael, J.A. | Limeira-de-Oliveira, F. | Takiya, D.M. | et al.; identifiedBy: Allan Paulo Moreira dos Santos; institutionCode: DZRJ; basisOfRecord: PreservedSpecimen

n. country: Brazil; stateProvince: Ceará; municipality: Ubajara; locality: Parque Nacional de Ubajara, Trilha Araticum, Rio das Minas na altura da trilha do teleférico; maximumElevationInMeters: 420; verbatimCoordinates: 349'58"S, 4053'53"W; samplingProtocol: Malaise intercept trap; verbatimEventDate: 20.iv.12; individualCount: 5; sex: male; lifeStage: adult; recordedBy: Rafael, J.A. | Limeira-de-Oliveira, F. | Takiya, D.M. | et al.; identifiedBy: Allan Paulo Moreira dos Santos; institutionCode: DZRJ; basisOfRecord: PreservedSpecimen

o. country: Brazil; stateProvince: Ceará; municipality: Ubajara; locality: Parque Nacional de Ubajara, Trilha Araticum, Rio das Minas na altura da trilha do teleférico; maximumElevationInMeters: 420; verbatimCoordinates: 349'58"S, 4053'53"W; samplingProtocol: Malaise intercept trap; verbatimEventDate: 20.iv.12; individualCount: 52; sex: female; lifeStage: adult; recordedBy: Rafael, J.A. | Limeira-de-Oliveira, F. | Takiya, D.M. | et al.; identifiedBy: Allan Paulo Moreira dos Santos; institutionCode: DZRJ; basisOfRecord: PreservedSpecimen

p. country: Brazil; stateProvince: Ceará; municipality: Ubajara; locality: Parque Nacional de Ubajara, Trilha Araticum, Rio das Minas; maximumElevationInMeters: 524; verbatimCoordinates: $3^{\circ} 50^{\prime} 3$ "S, $40^{\circ} 54^{\prime} 18 \mathrm{WW}$; samplingProtocol: Pennsylvania light trap; verbatimEventDate: 21.iv.12; individualCount: 11; sex: male; lifeStage: adult; recordedBy: Takiya, D.M. | Câmara, J.T.; identifiedBy: Allan Paulo Moreira dos Santos; institutionCode: DZRJ; basisOfRecord: PreservedSpecimen

q. country: Brazil; stateProvince: Ceará; municipality: Ubajara; locality: Parque Nacional de Ubajara, Trilha Araticum, Rio das Minas; maximumElevationInMeters: 524; verbatimCoordinates: $3^{\circ} 50^{\prime} 3$ "S, $40^{\circ} 54^{\prime} 18^{\prime \prime} \mathrm{W}$; samplingProtocol: Pennsylvania light trap; verbatimEventDate: 21.iv.12; individualCount: 25; sex: female; lifeStage: adult; recordedBy: Takiya, D.M. | Câmara, J.T.; identifiedBy: Allan Paulo Moreira dos Santos; institutionCode: DZRJ; basisOfRecord: PreservedSpecimen

r. country: Brazil; stateProvince: Ceará; municipality: Ubajara; locality: Parque Nacional de Ubajara, Trilha Araticum, Rio das Minas; maximumElevationInMeters: 524; verbatimCoordinates: $3^{\circ} 50^{\prime} 3$ "S, $40^{\circ} 54^{\prime} 18 \mathrm{~W}$; samplingProtocol: Pennsylvania light trap; verbatimEventDate: 22.iv.12; individualCount: 2; sex: male; lifeStage: adult; recordedBy: Takiya, D.M. | Somavilla, A.; identifiedBy: Allan Paulo Moreira dos Santos; institutionCode: DZRJ; basisOfRecord: PreservedSpecimen

s. $\quad$ country: Brazil; stateProvince: Ceará; municipality: Ubajara; locality: Parque Nacional de Ubajara, Trilha Araticum, Rio das Minas; maximumElevationInMeters: 524; verbatimCoordinates: $3^{\circ} 50^{\prime} 3$ "S, $40^{\circ} 54^{\prime} 18 \mathrm{WW}$; samplingProtocol: Pennsylvania light trap; verbatimEventDate: 22.iv.12; individualCount: 11; sex: female; lifeStage: adult; recordedBy: Takiya, D.M. | Somavilla, A.; identifiedBy: Allan Paulo Moreira dos Santos; institutionCode: DZRJ; basisOfRecord: PreservedSpecimen

Distribution: Colombia. Venezuela. Guyana. Brazil: PA, PI, CE, GO, MG, DF, ES, RJ. Ecuador. Peru. Bolivia. Paraguay. Argentina. 


\section{Genus Macrostemum Kolenati, 1859}

\section{Macrostemum hyalinum (F.J. Pictet, 1836)}

\section{Materials}

a. country: Brazil; stateProvince: Ceará; municipality: Ubajara; locality: Parque Nacional de Ubajara, Rio Cafundó, pouco acima da cachoeira; maximumElevationInMeters: 795; verbatimCoordinates: $3^{\circ} 50^{\prime} 13^{\prime \prime} \mathrm{S}, 40^{\circ} 54^{\prime} 35^{\prime \prime} \mathrm{W}$; samplingProtocol: Malaise intercept trap; verbatimEventDate: 1.ii.13; individualCount: 5; sex: male; lifeStage: adult; recordedBy: Limeira-de-Oliveira | et al.; identifiedBy: Allan Paulo Moreira dos Santos; institutionCode: DZRJ; basisOfRecord: PreservedSpecimen

b. country: Brazil; stateProvince: Ceará; municipality: Ubajara; locality: Parque Nacional de Ubajara, Rio Cafundó, pouco acima da cachoeira; maximumElevationInMeters: 795; verbatimCoordinates: 350'13"S, 4054'35"W; samplingProtocol: Malaise intercept trap; verbatimEventDate: 1.ii.13; individualCount: 4; sex: female; lifeStage: adult; recordedBy: Limeira-de-Oliveira | et al.; identifiedBy: Allan Paulo Moreira dos Santos; institutionCode: DZRJ; basisOfRecord: PreservedSpecimen

c. country: Brazil; stateProvince: Ceará; municipality: Ubajara; locality: Parque Nacional de Ubajara, Rio Cafundó, pouco acima da cachoeira; maximumElevationInMeters: 795; verbatimCoordinates: $3^{\circ} 50^{\prime} 13^{\prime \prime S}$, 4054'35"W; samplingProtocol: Malaise intercept trap; verbatimEventDate: 1.xii.12; individualCount: 1; sex: male; lifeStage: adult; recordedBy: Limeira-de-Oliveira | et al.; identifiedBy: Allan Paulo Moreira dos Santos; institutionCode: DZRJ; basisOfRecord: PreservedSpecimen

d. country: Brazil; stateProvince: Ceará; municipality: Ubajara; locality: Parque Nacional de Ubajara, Rio Cafundó, pouco acima da cachoeira; maximumElevationInMeters: 795; verbatimCoordinates: $3^{\circ} 50^{\prime} 13^{\prime \prime} S$, 4054'35"W; samplingProtocol: Malaise intercept trap; verbatimEventDate: 1.xii.12; individualCount: 6; sex: female; lifeStage: adult; recordedBy: Limeira-de-Oliveira | et al.; identifiedBy: Allan Paulo Moreira dos Santos; institutionCode: DZRJ; basisOfRecord: PreservedSpecimen

e. $\quad$ country: Brazil; stateProvince: Ceará; municipality: Ubajara; locality: Parque Nacional de Ubajara, Rio Cafundó, pouco acima da cachoeira; maximumElevationInMeters: 795; verbatimCoordinates: 350'13"S, 4054'35"W; samplingProtocol: Malaise intercept trap; verbatimEventDate: 13.xi.12; individualCount: 8; sex: male; lifeStage: adult; recordedBy: Limeira-de-Oliveira | et al.; identifiedBy: Allan Paulo Moreira dos Santos; institutionCode: DZRJ; basisOfRecord: PreservedSpecimen

f. country: Brazil; stateProvince: Ceará; municipality: Ubajara; locality: Parque Nacional de Ubajara, Rio Cafundó, pouco acima da cachoeira; maximumElevationInMeters: 795; verbatimCoordinates: 350'13"S, 4054'35"W; samplingProtocol: Malaise intercept trap; verbatimEventDate: 13.xi.12; individualCount: 5; sex: female; lifeStage: adult; recordedBy: Limeira-de-Oliveira | et al.; identifiedBy: Allan Paulo Moreira dos Santos; institutionCode: DZRJ; basisOfRecord: PreservedSpecimen

g. country: Brazil; stateProvince: Ceará; municipality: Ubajara; locality: Parque Nacional de Ubajara, Rio Cafundó, pouco acima da cachoeira; maximumElevationInMeters: 795; verbatimCoordinates: $3^{\circ} 50^{\prime} 13^{\prime \prime} \mathrm{S}, 40^{\circ} 54^{\prime} 35^{\prime \prime} \mathrm{W}$; samplingProtocol: Malaise intercept trap; verbatimEventDate: 13.xi.12; individualCount: 2; sex: female; lifeStage: adult; recordedBy: Limeira-de-Oliveira | et al.; identifiedBy: Allan Paulo Moreira dos Santos; institutionCode: DZRJ; basisOfRecord: PreservedSpecimen

h. country: Brazil; stateProvince: Ceará; municipality: Ubajara; locality: Parque Nacional de Ubajara, Rio Cafundó, pouco acima da cachoeira; maximumElevationInMeters: 795; 
verbatimCoordinates: $3^{\circ} 50^{\prime} 13^{\prime \prime} \mathrm{S}, 40^{\circ} 54^{\prime} 35^{\prime \prime} \mathrm{W}$; samplingProtocol: Malaise intercept trap; verbatimEventDate: 13.xi.12; individualCount: 1; sex: female; lifeStage: adult; recordedBy: Limeira-de-Oliveira | et al.; identifiedBy: Allan Paulo Moreira dos Santos; institutionCode: DZRJ; basisOfRecord: PreservedSpecimen

i. country: Brazil; stateProvince: Ceará; municipality: Ubajara; locality: Parque Nacional de Ubajara, Trilha Samambaia, Rio Gameleira; maximumElevationInMeters: 874; verbatimCoordinates: $3^{\circ} 50^{\prime} 25^{\prime \prime}$ S, $40^{\circ} 54^{\prime} 19^{\prime \prime}$; samplingProtocol: Pennsylvania light trap; verbatimEventDate: 14.ii.13; individualCount: 1; sex: female; lifeStage: adult; recordedBy: Santos, A.P.M. | Takiya, D.M.; identifiedBy: Allan Paulo Moreira dos Santos; institutionCode: DZRJ; basisOfRecord: PreservedSpecimen

j. country: Brazil; stateProvince: Ceará; municipality: Ubajara; locality: Parque Nacional de Ubajara, Trilha Samambaia, Rio Gameleira; maximumElevationInMeters: 874; verbatimCoordinates: $3^{\circ} 50^{\prime 2} 25^{\prime} \mathrm{S}, 40^{\circ} 54^{\prime} 19^{\prime \prime W}$; samplingProtocol: Malaise intercept trap; verbatimEventDate: 14.ii.13; individualCount: 3; sex: male; lifeStage: adult; recordedBy: Rafael, J.A. | Limeira-de-Oliveira, F. | Takiya, D.M. | Santos, A.P.M. | et al.; identifiedBy: Allan Paulo Moreira dos Santos; institutionCode: DZRJ; basisOfRecord: PreservedSpecimen

k. country: Brazil; stateProvince: Ceará; municipality: Ubajara; locality: Parque Nacional de Ubajara, Trilha Samambaia, Rio Gameleira; maximumElevationInMeters: 874; verbatimCoordinates: $3^{\circ} 50^{\prime} 25^{\prime \prime S}, 40^{\circ} 54^{\prime} 19^{\prime \prime W}$; samplingProtocol: Malaise intercept trap; verbatimEventDate: 17.ii.13; individualCount: 2; sex: male; lifeStage: adult; recordedBy: Rafael, J.A. | Limeira-de-Oliveira, F. | Takiya, D.M. | Santos, A.P.M. | et al.; identifiedBy: Allan Paulo Moreira dos Santos; institutionCode: DZRJ; basisOfRecord: PreservedSpecimen

I. country: Brazil; stateProvince: Ceará; municipality: Ubajara; locality: Parque Nacional de Ubajara, Trilha Samambaia, Rio Gameleira; maximumElevationInMeters: 874; verbatimCoordinates: $3^{\circ} 50^{\prime 2} 25^{\prime} \mathrm{S}, 40^{\circ} 54^{\prime} 19^{\prime \prime W}$; samplingProtocol: Malaise intercept trap; verbatimEventDate: 17.ii.13; individualCount: 2; sex: female; lifeStage: adult; recordedBy: Rafael, J.A. | Limeira-de-Oliveira, F. | Takiya, D.M. | Santos, A.P.M. | et al.; identifiedBy: Allan Paulo Moreira dos Santos; institutionCode: DZRJ; basisOfRecord: PreservedSpecimen

m. country: Brazil; stateProvince: Ceará; municipality: Ubajara; locality: Parque Nacional de Ubajara, Rio Cafundó, pouco acima da cachoeira; maximumElevationInMeters: 795; verbatimCoordinates: $3^{\circ} 50^{\prime} 13^{\prime \prime}$ S, $40^{\circ} 54^{\prime} 35 " \mathrm{~W}$; samplingProtocol: Malaise intercept trap; verbatimEventDate: 18.xi.12; individualCount: 1; sex: male; lifeStage: adult; recordedBy: Limeira-de-Oliveira | et al.; identifiedBy: Allan Paulo Moreira dos Santos; institutionCode: DZRJ; basisOfRecord: PreservedSpecimen

n. country: Brazil; stateProvince: Ceará; municipality: Ubajara; locality: Parque Nacional de Ubajara, Rio Cafundó, pouco acima da cachoeira; maximumElevationInMeters: 795; verbatimCoordinates: $3^{\circ} 50^{\prime} 13^{\prime \prime S}, 40^{\circ} 54^{\prime} 35 " \mathrm{~W}$; samplingProtocol: Malaise intercept trap; verbatimEventDate: 18.xi.12; individualCount: 4; sex: male; lifeStage: adult; recordedBy: Limeira-de-Oliveira | et al.; identifiedBy: Allan Paulo Moreira dos Santos; institutionCode: DZRJ; basisOfRecord: PreservedSpecimen

o. country: Brazil; stateProvince: Ceará; municipality: Ubajara; locality: Parque Nacional de Ubajara, Trilha Samambaia, Rio Gameleira; maximumElevationInMeters: 874; verbatimCoordinates: $3^{\circ} 50^{\prime} 25^{\prime \prime S}, 40^{\circ} 54^{\prime} 19 " \mathrm{~W}$; samplingProtocol: Malaise intercept trap; verbatimEventDate: 20.iv.12; individualCount: 1; sex: male; lifeStage: adult; recordedBy: Rafael, J.A. | Limeira-de-Oliveira, F. | Takiya, D.M. | et al.; identifiedBy: Allan Paulo Moreira dos Santos; institutionCode: DZRJ; basisOfRecord: PreservedSpecimen 
p. country: Brazil; stateProvince: Ceará; municipality: Ubajara; locality: Parque Nacional de Ubajara, Trilha Samambaia, Rio Gameleira; maximumElevationInMeters: 874; verbatimCoordinates: $3^{\circ} 50^{\prime} 25^{\prime \prime S}, 40^{\circ} 54^{\prime} 19 " \mathrm{~W}$; samplingProtocol: Malaise intercept trap; verbatimEventDate: 20.iv.12; individualCount: 3; sex: female; lifeStage: adult; recordedBy: Rafael, J.A. | Limeira-de-Oliveira, F. | Takiya, D.M. | et al.; identifiedBy: Allan Paulo Moreira dos Santos; institutionCode: DZRJ; basisOfRecord: PreservedSpecimen

q. country: Brazil; stateProvince: Ceará; municipality: Ubajara; locality: Parque Nacional de Ubajara, Trilha Samambaia, Rio Gameleira; maximumElevationInMeters: 874; verbatimCoordinates: $3^{\circ} 50^{\prime 25} \mathrm{~S}$ S, $40^{\circ} 54^{\prime} 19 \mathrm{~W} \mathrm{~W}$; samplingProtocol: Malaise intercept trap; verbatimEventDate: 20.iv.12; individualCount: 1; sex: male; lifeStage: adult; recordedBy: Rafael, J.A. | Limeira-de-Oliveira, F. | Takiya, D.M. | et al.; identifiedBy: Allan Paulo Moreira dos Santos; institutionCode: DZRJ; basisOfRecord: PreservedSpecimen

r. country: Brazil; stateProvince: Ceará; municipality: Ubajara; locality: Parque Nacional de Ubajara, Trilha Samambaia, Rio Gameleira; maximumElevationInMeters: 874; verbatimCoordinates: $3^{\circ} 50$ '25"S, 4054'19"W; samplingProtocol: Malaise intercept trap; verbatimEventDate: 20.iv.12; individualCount: 2; sex: female; lifeStage: adult; recordedBy: Rafael, J.A. | Limeira-de-Oliveira, F. | Takiya, D.M. | et al.; identifiedBy: Allan Paulo Moreira dos Santos; institutionCode: DZRJ; basisOfRecord: PreservedSpecimen

Distribution: Colombia. Venezuela. Guyana. Brazil: PA, CE, PB, PE, MT, AC, BA, ES, SP, RJ, PR. Peru.

\section{Macrostemum ulmeri* (Banks, 1913)}

\section{Materials}

a. country: Brazil; stateProvince: Ceará; municipality: Ubajara; locality: Parque Nacional de Ubajara, Trilha Araticum, Rio das Minas; maximumElevationInMeters: 524; verbatimCoordinates: $3^{\circ} 50^{\prime} 3$ "S, $40^{\circ} 54^{\prime} 18 \mathrm{~W}$; samplingProtocol: Malaise intercept trap; verbatimEventDate: 17.ii.13; individualCount: 1; sex: male; lifeStage: adult; recordedBy: Rafael, J.A. | Limeira-de-Oliveira, F. | Takiya, D.M. | Santos, A.P.M. | et al.; identifiedBy: Allan Paulo Moreira dos Santos; institutionCode: DZRJ; basisOfRecord: PreservedSpecimen

b. $\quad$ country: Brazil; stateProvince: Ceará; municipality: Ubajara; locality: Parque Nacional de Ubajara, Trilha Araticum, Rio das Minas na altura da trilha do teleférico; maximumElevationInMeters: 420; verbatimCoordinates: $3^{\circ} 49^{\prime} 58^{\prime \prime S}, 40^{\circ} 53^{\prime} 53^{\prime \prime} \mathrm{W}$; samplingProtocol: Malaise intercept trap; verbatimEventDate: 20.iv.12; individualCount: 1; sex: female; lifeStage: adult; recordedBy: Rafael, J.A. | Limeira-de-Oliveira, F. | Takiya, D.M. | et al.; identifiedBy: Allan Paulo Moreira dos Santos; institutionCode: DZRJ; basisOfRecord: PreservedSpecimen

c. country: Brazil; stateProvince: Ceará; municipality: Ubajara; locality: Parque Nacional de Ubajara, Rio Cafundó, pouco acima da cachoeira; maximumElevationInMeters: 795; verbatimCoordinates: $3^{\circ} 50^{\prime} 13^{\prime \prime} \mathrm{S}, 40^{\circ} 54^{\prime} 35 \mathrm{~W} \mathrm{~W}$; samplingProtocol: Malaise intercept trap; verbatimEventDate: 1.xii.12; individualCount: 1; sex: male; lifeStage: adult; recordedBy: Limeira-de-Oliveira | et al.; identifiedBy: Allan Paulo Moreira dos Santos; institutionCode: DZRJ; basisOfRecord: PreservedSpecimen

d. country: Brazil; stateProvince: Ceará; municipality: Ubajara; locality: Parque Nacional de Ubajara, Rio Cafundó, pouco acima da cachoeira; maximumElevationInMeters: 795; verbatimCoordinates: $3^{\circ} 50^{\prime} 13^{\prime \prime} \mathrm{S}, 40^{\circ} 54^{\prime} 35 " \mathrm{~W}$; samplingProtocol: Malaise intercept trap; verbatimEventDate: 1.xii.12; individualCount: 6; sex: female; lifeStage: adult; recordedBy: 
Limeira-de-Oliveira | et al.; identifiedBy: Allan Paulo Moreira dos Santos; institutionCode: DZRJ; basisOfRecord: PreservedSpecimen

e. $\quad$ country: Brazil; stateProvince: Ceará; municipality: Ubajara; locality: Parque Nacional de Ubajara, Rio Cafundó, pouco acima da cachoeira; maximumElevationInMeters: 795; verbatimCoordinates: $3^{\circ} 50^{\prime} 13^{\prime \prime} \mathrm{S}, 40^{\circ} 54^{\prime} 35^{\prime \prime} \mathrm{W}$; samplingProtocol: Malaise intercept trap; verbatimEventDate: 13.xi.12; individualCount: 8; sex: male; lifeStage: adult; recordedBy: Limeira-de-Oliveira | et al.; identifiedBy: Allan Paulo Moreira dos Santos; institutionCode: DZRJ; basisOfRecord: PreservedSpecimen

f. country: Brazil; stateProvince: Ceará; municipality: Ubajara; locality: Parque Nacional de Ubajara, Rio Cafundó, pouco acima da cachoeira; maximumElevationInMeters: 795; verbatimCoordinates: $3^{\circ} 50^{\prime} 13^{\prime \prime S}$, 4054'35"W; samplingProtocol: Malaise intercept trap; verbatimEventDate: 13.xi.12; individualCount: 5; sex: female; lifeStage: adult; recordedBy: Limeira-de-Oliveira | et al.; identifiedBy: Allan Paulo Moreira dos Santos; institutionCode: DZRJ; basisOfRecord: PreservedSpecimen

g. country: Brazil; stateProvince: Ceará; municipality: Ubajara; locality: Parque Nacional de Ubajara, Rio Cafundó, pouco acima da cachoeira; maximumElevationInMeters: 795; verbatimCoordinates: $3^{\circ} 50^{\prime} 13^{\prime \prime} \mathrm{S}, 40^{\circ} 54^{\prime} 35 \mathrm{WW}$; samplingProtocol: Malaise intercept trap; verbatimEventDate: 13.xi.12; individualCount: 2; sex: female; lifeStage: adult; recordedBy: Limeira-de-Oliveira | et al.; identifiedBy: Allan Paulo Moreira dos Santos; institutionCode: DZRJ; basisOfRecord: PreservedSpecimen

h. country: Brazil; stateProvince: Ceará; municipality: Ubajara; locality: Parque Nacional de Ubajara, Rio Cafundó, pouco acima da cachoeira; maximumElevationInMeters: 795; verbatimCoordinates: $3^{\circ} 50^{\prime} 13^{\prime \prime} \mathrm{S}, 40^{\circ} 54^{\prime} 35 \mathrm{WW}$; samplingProtocol: Malaise intercept trap; verbatimEventDate: 13.xi.12; individualCount: 1; sex: female; lifeStage: adult; recordedBy: Limeira-de-Oliveira | et al.; identifiedBy: Allan Paulo Moreira dos Santos; institutionCode: DZRJ; basisOfRecord: PreservedSpecimen

i. country: Brazil; stateProvince: Ceará; municipality: Ubajara; locality: Parque Nacional de Ubajara, Trilha Samambaia, Rio Gameleira; maximumElevationInMeters: 874; verbatimCoordinates: $3^{\circ} 50^{\prime} 25^{\prime \prime}$ S, $40^{\circ} 54^{\prime} 19^{\prime \prime} \mathrm{W}$; samplingProtocol: Pennsylvania light trap; verbatimEventDate: 14.ii.13; individualCount: 1; sex: female; lifeStage: adult; recordedBy: Santos, A.P.M. | Takiya, D.M.; identifiedBy: Allan Paulo Moreira dos Santos; institutionCode: DZRJ; basisOfRecord: PreservedSpecimen

j. country: Brazil; stateProvince: Ceará; municipality: Ubajara; locality: Parque Nacional de Ubajara, Trilha Samambaia, Rio Gameleira; maximumElevationInMeters: 874; verbatimCoordinates: $3^{\circ} 50^{\prime 25}$ "S, 4054'19"W; samplingProtocol: Malaise intercept trap; verbatimEventDate: 14.ii.13; individualCount: 3; sex: male; lifeStage: adult; recordedBy: Rafael, J.A. | Limeira-de-Oliveira, F. | Takiya, D.M. | Santos, A.P.M. | et al.; identifiedBy: Allan Paulo Moreira dos Santos; institutionCode: DZRJ; basisOfRecord: PreservedSpecimen

k. country: Brazil; stateProvince: Ceará; municipality: Ubajara; locality: Parque Nacional de Ubajara, Trilha Samambaia, Rio Gameleira; maximumElevationInMeters: 874; verbatimCoordinates: $3^{\circ} 50^{\prime} 25^{\prime \prime} \mathrm{S}, 40^{\circ} 54^{\prime} 19^{\prime \prime W}$; samplingProtocol: Malaise intercept trap; verbatimEventDate: 17.ii.13; individualCount: 2; sex: male; lifeStage: adult; recordedBy: Rafael, J.A. | Limeira-de-Oliveira, F. | Takiya, D.M. | Santos, A.P.M. | et al.; identifiedBy: Allan Paulo Moreira dos Santos; institutionCode: DZRJ; basisOfRecord: PreservedSpecimen

I. country: Brazil; stateProvince: Ceará; municipality: Ubajara; locality: Parque Nacional de Ubajara, Trilha Samambaia, Rio Gameleira; maximumElevationInMeters: 874; verbatimCoordinates: $3^{\circ} 50^{\prime} 25^{\prime \prime} \mathrm{S}, 40^{\circ} 54^{\prime} 19^{\prime \prime W}$; samplingProtocol: Malaise intercept trap; verbatimEventDate: 17.ii.13; individualCount: 2; sex: female; lifeStage: adult; recordedBy: 
Rafael, J.A. | Limeira-de-Oliveira, F. | Takiya, D.M. | Santos, A.P.M. | et al.; identifiedBy: Allan Paulo Moreira dos Santos; institutionCode: DZRJ; basisOfRecord:

PreservedSpecimen

m. country: Brazil; stateProvince: Ceará; municipality: Ubajara; locality: Parque Nacional de Ubajara, Rio Cafundó, pouco acima da cachoeira; maximumElevationInMeters: 795; verbatimCoordinates: $3^{\circ} 50^{\prime} 13^{\prime \prime S}$, $40^{\circ} 54^{\prime} 35 " \mathrm{~W}$; samplingProtocol: Malaise intercept trap; verbatimEventDate: 18.xi.12; individualCount: 1; sex: male; lifeStage: adult; recordedBy: Limeira-de-Oliveira | et al.; identifiedBy: Allan Paulo Moreira dos Santos; institutionCode: DZRJ; basisOfRecord: PreservedSpecimen

n. country: Brazil; stateProvince: Ceará; municipality: Ubajara; locality: Parque Nacional de Ubajara, Rio Cafundó, pouco acima da cachoeira; maximumElevationInMeters: 795; verbatimCoordinates: $3^{\circ} 50^{\prime} 13^{\prime \prime S}$, 4054'35"W; samplingProtocol: Malaise intercept trap; verbatimEventDate: 18.xi.12; individualCount: 4; sex: male; lifeStage: adult; recordedBy: Limeira-de-Oliveira | et al.; identifiedBy: Allan Paulo Moreira dos Santos; institutionCode: DZRJ; basisOfRecord: PreservedSpecimen

o. country: Brazil; stateProvince: Ceará; municipality: Ubajara; locality: Parque Nacional de Ubajara, Trilha Samambaia, Rio Gameleira; maximumElevationInMeters: 874; verbatimCoordinates: $3^{\circ} 50^{\prime} 25^{\prime \prime S}, 40^{\circ} 54^{\prime} 19 " \mathrm{~W}$; samplingProtocol: Malaise intercept trap; verbatimEventDate: 20.iv.12; individualCount: 1; sex: male; lifeStage: adult; recordedBy: Rafael, J.A. | Limeira-de-Oliveira, F. | Takiya, D.M. | et al.; identifiedBy: Allan Paulo Moreira dos Santos; institutionCode: DZRJ; basisOfRecord: PreservedSpecimen

p. country: Brazil; stateProvince: Ceará; municipality: Ubajara; locality: Parque Nacional de Ubajara, Trilha Samambaia, Rio Gameleira; maximumElevationInMeters: 874; verbatimCoordinates: $3^{\circ} 50^{\prime} 25^{\prime \prime}$ S, $40^{\circ} 54^{\prime} 19 " \mathrm{~W}$; samplingProtocol: Malaise intercept trap; verbatimEventDate: 20.iv.12; individualCount: 3; sex: female; lifeStage: adult; recordedBy: Rafael, J.A. | Limeira-de-Oliveira, F. | Takiya, D.M. | et al.; identifiedBy: Allan Paulo Moreira dos Santos; institutionCode: DZRJ; basisOfRecord: PreservedSpecimen

q. country: Brazil; stateProvince: Ceará; municipality: Ubajara; locality: Parque Nacional de Ubajara, Trilha Samambaia, Rio Gameleira; maximumElevationInMeters: 874; verbatimCoordinates: $3^{\circ} 50^{\prime} 25 " \mathrm{~S}, 40^{\circ} 54^{\prime} 19^{\prime \prime} \mathrm{W}$; samplingProtocol: Malaise intercept trap; verbatimEventDate: 20.iv.12; individualCount: 1; sex: male; lifeStage: adult; recordedBy: Rafael, J.A. | Limeira-de-Oliveira, F. | Takiya, D.M. | et al.; identifiedBy: Allan Paulo Moreira dos Santos; institutionCode: DZRJ; basisOfRecord: PreservedSpecimen

r. country: Brazil; stateProvince: Ceará; municipality: Ubajara; locality: Parque Nacional de Ubajara, Trilha Samambaia, Rio Gameleira; maximumElevationInMeters: 874; verbatimCoordinates: $3^{\circ} 50^{\prime} 25^{\prime \prime S}, 40^{\circ} 54^{\prime} 19 " \mathrm{~W}$; samplingProtocol: Malaise intercept trap; verbatimEventDate: 20.iv.12; individualCount: 2; sex: female; lifeStage: adult; recordedBy: Rafael, J.A. | Limeira-de-Oliveira, F. | Takiya, D.M. | et al.; identifiedBy: Allan Paulo Moreira dos Santos; institutionCode: DZRJ; basisOfRecord: PreservedSpecimen

Distribution: Honduras. Costa Rica. Panama. Colombia. Suriname. Brazil: PA, AM, PI!, CE!, MT, AC, RO, SP. Ecuador. Peru.

Notes: New species record for CE.

\section{Genus Smicridea McLachlan, 1871}




\section{Smicridea (Smicridea) bivittata* (Hagen, 1861)}

\section{Materials}

a. country: Brazil; stateProvince: Ceará; municipality: Ubajara; locality: Parque Nacional de Ubajara, Trilha Araticum, Rio das Minas; maximumElevationInMeters: 524; verbatimCoordinates: $3^{\circ} 50^{\prime} 3$ "S, $40^{\circ} 54^{\prime} 18$ "W; samplingProtocol: Malaise intercept trap; verbatimEventDate: 17.ii.13; individualCount: 1; sex: male; lifeStage: adult; recordedBy: Rafael, J.A. | Limeira-de-Oliveira, F. | Takiya, D.M. | Santos, A.P.M. | et al.; identifiedBy: Allan Paulo Moreira dos Santos; institutionCode: DZRJ; basisOfRecord: PreservedSpecimen

b. $\quad$ country: Brazil; stateProvince: Ceará; municipality: Ubajara; locality: Parque Nacional de Ubajara, Trilha Araticum, Rio das Minas na altura da trilha do teleférico; maximumElevationInMeters: 420; verbatimCoordinates: $3^{\circ} 49^{\prime} 58^{\prime \prime} \mathrm{S}, 40^{\circ} 53^{\prime} 53^{\prime \prime} \mathrm{W}$; samplingProtocol: Malaise intercept trap; verbatimEventDate: 20.iv.12; individualCount: 1; sex: male; lifeStage: adult; recordedBy: Rafael, J.A. | Limeira-de-Oliveira, F. | Takiya, D.M. | et al.; identifiedBy: Allan Paulo Moreira dos Santos; institutionCode: DZRJ; basisOfRecord: PreservedSpecimen

c. country: Brazil; stateProvince: Ceará; municipality: Ubajara; locality: Parque Nacional de Ubajara, Trilha Samambaia, Rio Gameleira; maximumElevationInMeters: 874; verbatimCoordinates: $3^{\circ} 50^{\prime} 25^{\prime \prime}$ S, $40^{\circ} 54^{\prime} 19^{\prime \prime} \mathrm{W}$; samplingProtocol: Pennsylvania light trap; verbatimEventDate: 21.iv.12; individualCount: 8; sex: male; lifeStage: adult; recordedBy: Takiya, D.M. | Câmara, J.T.; identifiedBy: Allan Paulo Moreira dos Santos; institutionCode: DZRJ; basisOfRecord: PreservedSpecimen

d. country: Brazil; stateProvince: Ceará; municipality: Ubajara; locality: Parque Nacional de Ubajara, Trilha Samambaia, Rio Gameleira; maximumElevationInMeters: 874; verbatimCoordinates: $3^{\circ} 50^{\prime 2} 25^{\prime \prime}$, $40^{\circ} 54^{\prime} 19^{\prime \prime} \mathrm{W}$; samplingProtocol: Pennsylvania light trap; verbatimEventDate: 21.iv.12; individualCount: 6; sex: female; lifeStage: adult; recordedBy: Takiya, D.M. | Câmara, J.T.; identifiedBy: Allan Paulo Moreira dos Santos; institutionCode: DZRJ; basisOfRecord: PreservedSpecimen

e. $\quad$ country: Brazil; stateProvince: Ceará; municipality: Ubajara; locality: Parque Nacional de Ubajara, Trilha Samambaia, Rio Gameleira; maximumElevationInMeters: 874; verbatimCoordinates: $3^{\circ} 50^{\prime} 25 " \mathrm{~S}, 40^{\circ} 54^{\prime} 19^{\prime \prime} \mathrm{W}$; samplingProtocol: Pennsylvania light trap; verbatimEventDate: 21.iv.12; individualCount: 18; sex: male; lifeStage: adult; recordedBy: Takiya, D.M. | Câmara, J.T.; identifiedBy: Allan Paulo Moreira dos Santos; institutionCode: DZRJ; basisOfRecord: PreservedSpecimen

f. country: Brazil; stateProvince: Ceará; municipality: Ubajara; locality: Parque Nacional de Ubajara, Trilha Samambaia, Rio Gameleira; maximumElevationInMeters: 874; verbatimCoordinates: $3^{\circ} 50^{\prime} 25^{\prime \prime}$ S, $40^{\circ} 54^{\prime} 19^{\prime \prime W}$; samplingProtocol: Pennsylvania light trap; verbatimEventDate: 21.iv.12; individualCount: 10; sex: female; lifeStage: adult; recordedBy: Takiya, D.M. | Câmara, J.T.; identifiedBy: Allan Paulo Moreira dos Santos; institutionCode: DZRJ; basisOfRecord: PreservedSpecimen

g. country: Brazil; stateProvince: Ceará; municipality: Ubajara; locality: Parque Nacional de Ubajara, Rio Cafundó, pouco acima da cachoeira; maximumElevationInMeters: 795; verbatimCoordinates: $3^{\circ} 50^{\prime} 13^{\prime \prime S}, 40^{\circ} 54^{\prime} 35$ "W; samplingProtocol: Malaise intercept trap; verbatimEventDate: 21.iv.12; individualCount: 2; sex: male; lifeStage: adult; recordedBy: Rafael, J.A. | Limeira-de-Oliveira, F. | Takiya, D.M. | et al.; identifiedBy: Allan Paulo Moreira dos Santos; institutionCode: DZRJ; basisOfRecord: PreservedSpecimen

h. country: Brazil; stateProvince: Ceará; municipality: Ubajara; locality: Parque Nacional de Ubajara, Trilha Araticum, Rio das Minas; maximumElevationInMeters: 524; verbatimCoordinates: $3^{\circ} 50^{\prime} 3$ "S, $40^{\circ} 54^{\prime} 18$ "W; samplingProtocol: Pennsylvania light trap; 
verbatimEventDate: 22.iv.12; individualCount: 1; sex: male; lifeStage: adult; recordedBy: Takiya, D.M. | Somavilla, A.; identifiedBy: Allan Paulo Moreira dos Santos; institutionCode: DZRJ; basisOfRecord: PreservedSpecimen

i. country: Brazil; stateProvince: Ceará; municipality: Ubajara; locality: Parque Nacional de Ubajara, Trilha Araticum, Rio das Minas; maximumElevationInMeters: 524; verbatimCoordinates: $3^{\circ} 50$ '3"S, 4054'18"W; samplingProtocol: Pennsylvania light trap; verbatimEventDate: 22.iv.12; individualCount: 1; sex: female; lifeStage: adult; recordedBy: Takiya, D.M. | Somavilla, A.; identifiedBy: Allan Paulo Moreira dos Santos; institutionCode: DZRJ; basisOfRecord: PreservedSpecimen

j. country: Brazil; stateProvince: Ceará; municipality: Ubajara; locality: Parque Nacional de Ubajara, Rio Cafundó, pouco acima da cachoeira; maximumElevationInMeters: 795; verbatimCoordinates: $3^{\circ} 50^{\prime} 13^{\prime \prime} \mathrm{S}, 40^{\circ} 54^{\prime} 35$ "W; samplingProtocol: Pennsylvania light trap; verbatimEventDate: 24.iv.12; individualCount: 1; sex: male; lifeStage: adult; recordedBy: Takiya, D.M. | Rafael, J.A.; identifiedBy: Allan Paulo Moreira dos Santos; institutionCode: DZRJ; basisOfRecord: PreservedSpecimen

Distribution: Mexico. Guatemala. Honduras. El Salvador. Costa Rica. Trinidad and Tobago. Venezuela. Suriname. Brazil: PA, PI!, CE!, MG, SP. Ecuador.

Notes: New species record for Northeastern Brazil.

\section{Smicridea (Smicridea) franciscana Rocha, Dumas \& Nessimian, 2016}

\section{Materials}

a. country: Brazil; stateProvince: Ceará; municipality: Ubajara; locality: Parque Nacional de Ubajara, Trilha Araticum, Rio das Minas na altura da trilha do teleférico; maximumElevationInMeters: 420; verbatimCoordinates: $3^{\circ} 49^{\prime} 58^{\prime \prime S}, 40^{\circ} 53^{\prime} 53^{\prime \prime} \mathrm{W}$; samplingProtocol: Malaise intercept trap; verbatimEventDate: 14.ii.13; individualCount: 1; sex: male; lifeStage: adult; recordedBy: Rafael, J.A. | Limeira-de-Oliveira, F. | Takiya, D.M. | Santos, A.P.M. | et al.; identifiedBy: Allan Paulo Moreira dos Santos; institutionCode: DZRJ; basisOfRecord: PreservedSpecimen

b. country: Brazil; stateProvince: Ceará; municipality: Ubajara; locality: Parque Nacional de Ubajara, Rio das Minas, próximo ao Portão Araticum; maximumElevationInMeters: 328; verbatimCoordinates: $3^{\circ} 49^{\prime} 32.6^{\prime \prime S}, 40^{\circ} 53^{\prime} 32.8^{\prime \prime} \mathrm{W}$; samplingProtocol: Malaise intercept trap; verbatimEventDate: 17.ii.13; individualCount: 3; sex: male; lifeStage: adult; recordedBy: Rafael, J.A. | Limeira-de-Oliveira, F. | Takiya, D.M. | Santos, A.P.M. | et al.; identifiedBy: Allan Paulo Moreira dos Santos; institutionCode: DZRJ; basisOfRecord: PreservedSpecimen

c. country: Brazil; stateProvince: Ceará; municipality: Ubajara; locality: Parque Nacional de Ubajara, Trilha Samambaia, Rio Gameleira; maximumElevationInMeters: 874; verbatimCoordinates: $3^{\circ} 50^{\prime 2} 25^{\prime \prime}$, $40^{\circ} 54^{\prime} 19^{\prime \prime} \mathrm{W}$; samplingProtocol: Pennsylvania light trap; verbatimEventDate: 21.iv.12; individualCount: 8; sex: male; lifeStage: adult; recordedBy: Takiya, D.M. | Câmara, J.T.; identifiedBy: Allan Paulo Moreira dos Santos; institutionCode: DZRJ; basisOfRecord: PreservedSpecimen

d. country: Brazil; stateProvince: Ceará; municipality: Ubajara; locality: Parque Nacional de Ubajara, Trilha Samambaia, Rio Gameleira; maximumElevationInMeters: 874; verbatimCoordinates: $3^{\circ} 50^{\prime} 25 " \mathrm{~S}, 40^{\circ} 54^{\prime} 19^{\prime \prime} \mathrm{W}$; sampling Protocol: Pennsylvania light trap; verbatimEventDate: 21.iv.12; individualCount: 6; sex: female; lifeStage: adult; recordedBy: 
Takiya, D.M. | Câmara, J.T.; identifiedBy: Allan Paulo Moreira dos Santos; institutionCode: DZRJ; basisOfRecord: PreservedSpecimen

e. $\quad$ country: Brazil; stateProvince: Ceará; municipality: Ubajara; locality: Parque Nacional de Ubajara, Trilha Samambaia, Rio Gameleira; maximumElevationInMeters: 874; verbatimCoordinates: $3^{\circ} 50^{\prime} 25^{\prime \prime}$ S, $40^{\circ} 54^{\prime} 19 " \mathrm{~W}$; samplingProtocol: Pennsylvania light trap; verbatimEventDate: 21.iv.12; individualCount: 18; sex: male; lifeStage: adult; recordedBy: Takiya, D.M. | Câmara, J.T.; identifiedBy: Allan Paulo Moreira dos Santos; institutionCode: DZRJ; basisOfRecord: PreservedSpecimen

f. country: Brazil; stateProvince: Ceará; municipality: Ubajara; locality: Parque Nacional de Ubajara, Trilha Samambaia, Rio Gameleira; maximumElevationInMeters: 874; verbatimCoordinates: $3^{\circ} 50^{\prime} 25 " \mathrm{~S}, 40^{\circ} 54^{\prime} 19 " \mathrm{~W}$; sampling Protocol: Pennsylvania light trap; verbatimEventDate: 21.iv.12; individualCount: 10; sex: female; lifeStage: adult; recordedBy: Takiya, D.M. | Câmara, J.T.; identifiedBy: Allan Paulo Moreira dos Santos; institutionCode: DZRJ; basisOfRecord: PreservedSpecimen

g. country: Brazil; stateProvince: Ceará; municipality: Ubajara; locality: Parque Nacional de Ubajara, Rio Cafundó, pouco acima da cachoeira; maximumElevationInMeters: 795; verbatimCoordinates: $3^{\circ} 50^{\prime} 13^{\prime \prime S}, 40^{\circ} 54^{\prime} 35 " \mathrm{~W}$; samplingProtocol: Malaise intercept trap; verbatimEventDate: 21.iv.12; individualCount: 2; sex: male; lifeStage: adult; recordedBy: Rafael, J.A. | Limeira-de-Oliveira, F. | Takiya, D.M. | et al.; identifiedBy: Allan Paulo Moreira dos Santos; institutionCode: DZRJ; basisOfRecord: PreservedSpecimen

h. country: Brazil; stateProvince: Ceará; municipality: Ubajara; locality: Parque Nacional de Ubajara, Trilha Araticum, Rio das Minas; maximumElevationInMeters: 524; verbatimCoordinates: $3^{\circ} 50^{\prime} 3$ "S, $40^{\circ} 54^{\prime} 18$ "W; samplingProtocol: Pennsylvania light trap; verbatimEventDate: 22.iv.12; individualCount: 1; sex: male; lifeStage: adult; recordedBy: Takiya, D.M. | Somavilla, A.; identifiedBy: Allan Paulo Moreira dos Santos; institutionCode: DZRJ; basisOfRecord: PreservedSpecimen

i. country: Brazil; stateProvince: Ceará; municipality: Ubajara; locality: Parque Nacional de Ubajara, Trilha Araticum, Rio das Minas; maximumElevationInMeters: 524; verbatimCoordinates: $3^{\circ} 50 ' 3$ "S, 4054'18"W; samplingProtocol: Pennsylvania light trap; verbatimEventDate: 22.iv.12; individualCount: 1; sex: female; lifeStage: adult; recordedBy: Takiya, D.M. | Somavilla, A.; identifiedBy: Allan Paulo Moreira dos Santos; institutionCode: DZRJ; basisOfRecord: PreservedSpecimen

j. country: Brazil; stateProvince: Ceará; municipality: Ubajara; locality: Parque Nacional de Ubajara, Rio Cafundó, pouco acima da cachoeira; maximumElevationInMeters: 795; verbatimCoordinates: $3^{\circ} 50^{\prime} 13^{\prime \prime} \mathrm{S}, 40^{\circ} 54^{\prime} 35 " \mathrm{~W}$; sampling Protocol: Pennsylvania light trap; verbatimEventDate: 24.iv.12; individualCount: 1; sex: male; lifeStage: adult; recordedBy: Takiya, D.M. | Rafael, J.A.; identifiedBy: Allan Paulo Moreira dos Santos; institutionCode: DZRJ; basisOfRecord: PreservedSpecimen

Distribution: Brazil: CE!, MG.

Notes: New species record for Northeastern Brazil. 


\section{Smicridea (Smicridea) sp. 2*}

\section{Materials}

a. country: Brazil; stateProvince: Ceará; municipality: Ubajara; locality: Parque Nacional de Ubajara, Rio Cafundó, pouco acima da cachoeira; maximumElevationInMeters: 795; verbatimCoordinates: $3^{\circ} 50^{\prime} 13^{\prime \prime} \mathrm{S}, 40^{\circ} 54^{\prime} 35 \mathrm{WW}$; samplingProtocol: Malaise intercept trap; verbatimEventDate: 1.ii.13; individualCount: 3; sex: male; lifeStage: adult; recordedBy: Limeira-de-Oliveira | et al.; identifiedBy: Allan Paulo Moreira dos Santos; institutionCode: DZRJ; basisOfRecord: PreservedSpecimen

b. country: Brazil; stateProvince: Ceará; municipality: Ubajara; locality: Parque Nacional de Ubajara, Rio Cafundó, pouco acima da cachoeira; maximumElevationInMeters: 795; verbatimCoordinates: $3^{\circ} 50^{\prime} 13^{\prime \prime S}$, 4054'35"W; samplingProtocol: Malaise intercept trap; verbatimEventDate: 1.ii.13; individualCount: 1; sex: female; lifeStage: adult; recordedBy: Limeira-de-Oliveira | et al.; identifiedBy: Allan Paulo Moreira dos Santos; institutionCode: DZRJ; basisOfRecord: PreservedSpecimen

c. country: Brazil; stateProvince: Ceará; municipality: Ubajara; locality: Parque Nacional de Ubajara, Rio Cafundó, pouco acima da cachoeira; maximumElevationInMeters: 795; verbatimCoordinates: $3^{\circ} 50^{\prime} 13^{\prime \prime} \mathrm{S}, 40^{\circ} 54^{\prime} 35^{\prime \prime} \mathrm{W}$; samplingProtocol: Malaise intercept trap; verbatimEventDate: 13.ix.12; individualCount: 1; sex: male; lifeStage: adult; recordedBy: Limeira-de-Oliveira | et al.; identifiedBy: Allan Paulo Moreira dos Santos; institutionCode: DZRJ; basisOfRecord: PreservedSpecimen

d. country: Brazil; stateProvince: Ceará; municipality: Ubajara; locality: Parque Nacional de Ubajara, Rio Cafundó, pouco acima da cachoeira; maximumElevationInMeters: 795; verbatimCoordinates: $3^{\circ} 50^{\prime} 13^{\prime \prime S}$, 4054'35"W; samplingProtocol: Malaise intercept trap; verbatimEventDate: 13.xi.12; individualCount: 8; sex: male; lifeStage: adult; recordedBy: Limeira-de-Oliveira | et al.; identifiedBy: Allan Paulo Moreira dos Santos; institutionCode: DZRJ; basisOfRecord: PreservedSpecimen

e. $\quad$ country: Brazil; stateProvince: Ceará; municipality: Ubajara; locality: Parque Nacional de Ubajara, Rio Cafundó, pouco acima da cachoeira; maximumElevationInMeters: 795; verbatimCoordinates: $3^{\circ} 50^{\prime} 13^{\prime \prime S}, 40^{\circ} 54^{\prime} 35 \mathrm{~W} \mathrm{~W}$; samplingProtocol: Malaise intercept trap; verbatimEventDate: 13.xi.12; individualCount: 5; sex: female; lifeStage: adult; recordedBy: Limeira-de-Oliveira | et al.; identifiedBy: Allan Paulo Moreira dos Santos; institutionCode: DZRJ; basisOfRecord: PreservedSpecimen

f. country: Brazil; stateProvince: Ceará; municipality: Ubajara; locality: Parque Nacional de Ubajara, Trilha Samambaia, Rio Gameleira; maximumElevationInMeters: 874; verbatimCoordinates: $3^{\circ} 50^{\prime} 25^{\prime \prime}$ S, $40^{\circ} 54^{\prime} 19^{\prime \prime} \mathrm{W}$; samplingProtocol: Malaise intercept trap; verbatimEventDate: 20.iv.12; individualCount: 28; sex: male; lifeStage: adult; recordedBy: Rafael, J.A. | Limeira-de-Oliveira, F. | Takiya, D.M. | et al.; identifiedBy: Allan Paulo Moreira dos Santos; institutionCode: DZRJ; basisOfRecord: PreservedSpecimen

g. country: Brazil; stateProvince: Ceará; municipality: Ubajara; locality: Parque Nacional de Ubajara, Trilha Samambaia, Rio Gameleira; maximumElevationInMeters: 874; verbatimCoordinates: $3^{\circ} 50^{\prime 2} 25^{\prime \prime S}, 40^{\circ} 54^{\prime} 19^{\prime \prime W}$; samplingProtocol: Malaise intercept trap; verbatimEventDate: 20.iv.12; individualCount: 48; sex: male; lifeStage: adult; recordedBy: Rafael, J.A. | Limeira-de-Oliveira, F. | Takiya, D.M. | et al.; identifiedBy: Allan Paulo Moreira dos Santos; institutionCode: DZRJ; basisOfRecord: PreservedSpecimen

Notes: Undescribed species. 


\section{Smicridea (Smicridea) sp. 3}

\section{Materials}

a. country: Brazil; stateProvince: Ceará; municipality: Ubajara; locality: Parque Nacional de Ubajara, Rio Cafundó, pouco acima da cachoeira; maximumElevationInMeters: 795; verbatimCoordinates: $3^{\circ} 50^{\prime} 13^{\prime \prime S}, 40^{\circ} 54^{\prime} 35 \mathrm{WW}$; samplingProtocol: Malaise intercept trap; verbatimEventDate: 13.xi.12; individualCount: 2; sex: male; lifeStage: adult; recordedBy: Limeira-de-Oliveira | et al.; identifiedBy: Allan Paulo Moreira dos Santos; institutionCode: DZRJ; basisOfRecord: PreservedSpecimen

b. country: Brazil; stateProvince: Ceará; municipality: Ubajara; locality: Parque Nacional de Ubajara, Rio Cafundó, pouco acima da cachoeira; maximumElevationInMeters: 795; verbatimCoordinates: $3^{\circ} 50^{\prime} 13^{\prime \prime S}$, 4054'35"W; samplingProtocol: Malaise intercept trap; verbatimEventDate: 13.xi.12; individualCount: 2; sex: female; lifeStage: adult; recordedBy: Limeira-de-Oliveira | et al.; identifiedBy: Allan Paulo Moreira dos Santos; institutionCode: DZRJ; basisOfRecord: PreservedSpecimen

c. country: Brazil; stateProvince: Ceará; municipality: Ubajara; locality: Parque Nacional de Ubajara, Trilha Araticum, Rio das Minas; maximumElevationInMeters: 524; verbatimCoordinates: $3^{\circ} 50^{\prime} 3$ "S, $40^{\circ} 54^{\prime} 18^{\prime \prime} \mathrm{W}$; samplingProtocol: Malaise intercept trap; verbatimEventDate: 14.ii.13; individualCount: 5; sex: male; lifeStage: adult; recordedBy: Rafael, J.A. | Limeira-de-Oliveira, F. | Takiya, D.M. | Santos, A.P.M. | et al.; identifiedBy: Allan Paulo Moreira dos Santos; institutionCode: DZRJ; basisOfRecord: PreservedSpecimen

d. country: Brazil; stateProvince: Ceará; municipality: Ubajara; locality: Parque Nacional de Ubajara, Trilha Araticum, Rio das Minas; maximumElevationInMeters: 524; verbatimCoordinates: $3^{\circ} 50^{\prime} 3$ "S, $40^{\circ} 54^{\prime} 18^{\prime \prime W}$; samplingProtocol: Malaise intercept trap; verbatimEventDate: 14.ii.13; individualCount: 1; sex: female; lifeStage: adult; recordedBy: Rafael, J.A. | Limeira-de-Oliveira, F. | Takiya, D.M. | Santos, A.P.M. | et al.; identifiedBy: Allan Paulo Moreira dos Santos; institutionCode: DZRJ; basisOfRecord: PreservedSpecimen

e. $\quad$ country: Brazil; stateProvince: Ceará; municipality: Ubajara; locality: Parque Nacional de Ubajara, Trilha Araticum, Rio das Minas na altura da trilha do teleférico; maximumElevationInMeters: 420; verbatimCoordinates: $3^{\circ} 49^{\prime} 58^{\prime \prime S}, 40^{\circ} 53^{\prime} 53^{\prime \prime} \mathrm{W}$;

samplingProtocol: Malaise intercept trap; verbatimEventDate: 14.ii.13; individualCount: 2; sex: male; lifeStage: adult; recordedBy: Rafael, J.A. | Limeira-de-Oliveira, F. | Takiya, D.M. | Santos, A.P.M. | et al.; identifiedBy: Allan Paulo Moreira dos Santos; institutionCode: DZRJ; basisOfRecord: PreservedSpecimen

f. country: Brazil; stateProvince: Ceará; municipality: Ubajara; locality: Parque Nacional de Ubajara, Trilha Araticum, Rio das Minas; maximumElevationInMeters: 524; verbatimCoordinates: $3^{\circ} 50^{\prime} 3$ "S, $40^{\circ} 54^{\prime} 18 \mathrm{~W}$; samplingProtocol: Malaise intercept trap; verbatimEventDate: 17.ii.13; individualCount: 1; sex: male; lifeStage: adult; recordedBy: Rafael, J.A. | Limeira-de-Oliveira, F. | Takiya, D.M. | Santos, A.P.M. | et al.; identifiedBy: Allan Paulo Moreira dos Santos; institutionCode: DZRJ; basisOfRecord: PreservedSpecimen

g. country: Brazil; stateProvince: Ceará; municipality: Ubajara; locality: Parque Nacional de Ubajara, Trilha Araticum, Rio das Minas; maximumElevationInMeters: 524; verbatimCoordinates: $3^{\circ} 50^{\prime} 3^{\prime \prime} \mathrm{S}, 40^{\circ} 54^{\prime} 18^{\prime \prime} \mathrm{W}$; samplingProtocol: Malaise intercept trap; verbatimEventDate: 17.ii.13; individualCount: 2; sex: female; lifeStage: adult; recordedBy: Rafael, J.A. | Limeira-de-Oliveira, F. | Takiya, D.M. | Santos, A.P.M. | et al.; identifiedBy: Allan Paulo Moreira dos Santos; institutionCode: DZRJ; basisOfRecord: PreservedSpecimen 
h. country: Brazil; stateProvince: Ceará; municipality: Ubajara; locality: Parque Nacional de Ubajara, Trilha Samambaia, Rio Gameleira; maximumElevationInMeters: 874; verbatimCoordinates: $3^{\circ} 50^{\prime} 25^{\prime \prime S}, 40^{\circ} 54^{\prime} 19 " \mathrm{~W}$; samplingProtocol: Malaise intercept trap; verbatimEventDate: 20.iv.12; individualCount: 1; sex: male; lifeStage: adult; recordedBy: Rafael, J.A. | Limeira-de-Oliveira, F. | Takiya, D.M. | et al.; identifiedBy: Allan Paulo Moreira dos Santos; institutionCode: DZRJ; basisOfRecord: PreservedSpecimen

i. country: Brazil; stateProvince: Ceará; municipality: Ubajara; locality: Parque Nacional de Ubajara, Trilha Samambaia, Rio Gameleira; maximumElevationInMeters: 874; verbatimCoordinates: $3^{\circ} 50^{\prime 25} \mathrm{~S}$ S, $40^{\circ} 54^{\prime} 19 \mathrm{~W} \mathrm{~W}$; samplingProtocol: Malaise intercept trap; verbatimEventDate: 20.iv.12; individualCount: 2; sex: male; lifeStage: adult; recordedBy: Rafael, J.A. | Limeira-de-Oliveira, F. | Takiya, D.M. | et al.; identifiedBy: Allan Paulo Moreira dos Santos; institutionCode: DZRJ; basisOfRecord: PreservedSpecimen

j. $\quad$ country: Brazil; stateProvince: Ceará; municipality: Ubajara; locality: Parque Nacional de Ubajara, Trilha Araticum, Rio das Minas na altura da trilha do teleférico; maximumElevationInMeters: 420; verbatimCoordinates: $3^{\circ} 49^{\prime} 58$ "S, 4053'53"W; samplingProtocol: Malaise intercept trap; verbatimEventDate: 20.iv.12; individualCount: 1; sex: male; lifeStage: adult; recordedBy: Rafael, J.A. | Limeira-de-Oliveira, F. | Takiya, D.M. | et al.; identifiedBy: Allan Paulo Moreira dos Santos; institutionCode: DZRJ; basisOfRecord: PreservedSpecimen

k. country: Brazil; stateProvince: Ceará; municipality: Ubajara; locality: Parque Nacional de Ubajara, Trilha Araticum, Rio das Minas na altura da trilha do teleférico; maximumElevationInMeters: 420; verbatimCoordinates: $3^{\circ} 49^{\prime} 58^{\prime \prime S}, 40^{\circ} 53^{\prime} 53^{\prime \prime} \mathrm{W}$; samplingProtocol: Malaise intercept trap; verbatimEventDate: 20.iv.12; individualCount: 1; sex: male; lifeStage: adult; recordedBy: Rafael, J.A. | Limeira-de-Oliveira, F. | Takiya, D.M. I et al.; identifiedBy: Allan Paulo Moreira dos Santos; institutionCode: DZRJ; basisOfRecord: PreservedSpecimen

I. country: Brazil; stateProvince: Ceará; municipality: Ubajara; locality: Parque Nacional de Ubajara, Rio Cafundó, pouco acima da cachoeira; maximumElevationInMeters: 795; verbatimCoordinates: $3^{\circ} 50^{\prime} 13^{\prime \prime} \mathrm{S}, 40^{\circ} 54^{\prime} 35 \mathrm{~W} \mathrm{~W}$; samplingProtocol: Malaise intercept trap; verbatimEventDate: 21.iv.12; individualCount: 1; sex: male; lifeStage: adult; recordedBy: Rafael, J.A. | Limeira-de-Oliveira, F. | Takiya, D.M. | et al.; identifiedBy: Allan Paulo Moreira dos Santos; institutionCode: DZRJ; basisOfRecord: PreservedSpecimen

Notes: Undescribed species.

\section{Family Hydroptilidae}

Notes: Family firstly recorded from CE in Souza et al. 2014b.

\section{Genus Alisotrichia Flint, 1964}

Notes: New genus record for CE. 


\section{Alisotrichia sp. 1}

\section{Materials}

a. country: Brazil; stateProvince: Ceará; municipality: Ubajara; locality: Parque Nacional de Ubajara, Rio Cafundó, pouco acima da cachoeira; maximumElevationInMeters: 795; verbatimCoordinates: $3^{\circ} 50^{\prime} 13^{\prime \prime S}, 40^{\circ} 54^{\prime} 35 \mathrm{WW}$; samplingProtocol: Malaise intercept trap; verbatimEventDate: 13.ix.12; individualCount: 23; sex: male; lifeStage: adult; recordedBy: Limeira-de-Oliveira | et al.; identifiedBy: Allan Paulo Moreira dos Santos; institutionCode: DZRJ; basisOfRecord: PreservedSpecimen

b. $\quad$ country: Brazil; stateProvince: Ceará; municipality: Ubajara; locality: Parque Nacional de Ubajara, Rio Cafundó, pouco acima da cachoeira; maximumElevationInMeters: 795; verbatimCoordinates: $3^{\circ} 50^{\prime} 13^{\prime \prime} \mathrm{S}, 40^{\circ} 54^{\prime} 35 \mathrm{WW}$; samplingProtocol: Malaise intercept trap; verbatimEventDate: 13.ix.12; individualCount: 10; sex: female; lifeStage: adult; recordedBy: Limeira-de-Oliveira | et al.; identifiedBy: Allan Paulo Moreira dos Santos; institutionCode: DZRJ; basisOfRecord: PreservedSpecimen

c. country: Brazil; stateProvince: Ceará; municipality: Ubajara; locality: Parque Nacional de Ubajara, Rio Cafundó, pouco acima da cachoeira; maximumElevationInMeters: 795; verbatimCoordinates: $3^{\circ} 50^{\prime} 13^{\prime \prime} \mathrm{S}, 40^{\circ} 54^{\prime} 35^{\prime \prime} \mathrm{W}$; samplingProtocol: Malaise intercept trap; verbatimEventDate: 13.ix.12; individualCount: 23; sex: male; lifeStage: adult; recordedBy: Limeira-de-Oliveira | et al.; identifiedBy: Allan Paulo Moreira dos Santos; institutionCode: DZRJ; basisOfRecord: PreservedSpecimen

d. country: Brazil; stateProvince: Ceará; municipality: Ubajara; locality: Parque Nacional de Ubajara, Rio Cafundó, pouco acima da cachoeira; maximumElevationInMeters: 795; verbatimCoordinates: $3^{\circ} 50^{\prime} 13^{\prime \prime} \mathrm{S}, 40^{\circ} 54^{\prime} 35 \mathrm{WW}$; samplingProtocol: Malaise intercept trap; verbatimEventDate: 13.ix.12; individualCount: 10; sex: female; lifeStage: adult; recordedBy: Limeira-de-Oliveira | et al.; identifiedBy: Allan Paulo Moreira dos Santos; institutionCode: DZRJ; basisOfRecord: PreservedSpecimen

e. country: Brazil; stateProvince: Ceará; municipality: Ubajara; locality: Parque Nacional de Ubajara, Rio Cafundó, pouco acima da cachoeira; maximumElevationInMeters: 795; verbatimCoordinates: $3^{\circ} 50^{\prime} 13^{\prime \prime} \mathrm{S}, 40^{\circ} 54^{\prime} 35^{\prime \prime} \mathrm{W}$; samplingProtocol: Malaise intercept trap; verbatimEventDate: 13.xi.12; individualCount: 1; sex: male; lifeStage: adult; recordedBy: Limeira-de-Oliveira | et al.; identifiedBy: Allan Paulo Moreira dos Santos; institutionCode: DZRJ; basisOfRecord: PreservedSpecimen

f. country: Brazil; stateProvince: Ceará; municipality: Ubajara; locality: Parque Nacional de Ubajara, Trilha Samambaia, Rio Gameleira; maximumElevationInMeters: 874; verbatimCoordinates: $3^{\circ} 50^{\prime} 25^{\prime \prime} \mathrm{S}, 40^{\circ} 54^{\prime} 19^{\prime \prime} \mathrm{W}$; samplingProtocol: Malaise intercept trap; verbatimEventDate: 20.iv.12; individualCount: 3; sex: male; lifeStage: adult; recordedBy: Rafael, J.A. | Limeira-de-Oliveira, F. | Takiya, D.M. | et al.; identifiedBy: Allan Paulo Moreira dos Santos; institutionCode: DZRJ; basisOfRecord: PreservedSpecimen

g. country: Brazil; stateProvince: Ceará; municipality: Ubajara; locality: Parque Nacional de Ubajara, Trilha Samambaia, Rio Gameleira; maximumElevationInMeters: 874; verbatimCoordinates: $3^{\circ} 50^{\prime} 25^{\prime \prime} \mathrm{S}, 40^{\circ} 54^{\prime} 19^{\prime \prime W}$; samplingProtocol: Malaise intercept trap; verbatimEventDate: 20.iv.12; individualCount: 4; sex: female; lifeStage: adult; recordedBy: Rafael, J.A. | Limeira-de-Oliveira, F. | Takiya, D.M. | et al.; identifiedBy: Allan Paulo Moreira dos Santos; institutionCode: DZRJ; basisOfRecord: PreservedSpecimen

h. country: Brazil; stateProvince: Ceará; municipality: Ubajara; locality: Parque Nacional de Ubajara, Trilha Samambaia, Rio Gameleira; maximumElevationInMeters: 874; verbatimCoordinates: $3^{\circ} 50^{\prime} 25^{\prime \prime S}, 40^{\circ} 54^{\prime} 19 " \mathrm{~W}$; samplingProtocol: Malaise intercept trap; verbatimEventDate: 20.iv.12; individualCount: 10; sex: male; lifeStage: adult; recordedBy: 
Rafael, J.A. | Limeira-de-Oliveira, F. | Takiya, D.M. | et al.; identifiedBy: Allan Paulo Moreira dos Santos; institutionCode: DZRJ; basisOfRecord: PreservedSpecimen

i. country: Brazil; stateProvince: Ceará; municipality: Ubajara; locality: Parque Nacional de Ubajara, Trilha Samambaia, Rio Gameleira; maximumElevationInMeters: 874; verbatimCoordinates: $3^{\circ} 50^{\prime 2} 25^{\prime \prime}$, $40^{\circ} 54^{\prime} 19^{\prime \prime} \mathrm{W}$; samplingProtocol: Malaise intercept trap; verbatimEventDate: $20 . i v .12$; individualCount: 10; sex: female; lifeStage: adult; recordedBy: Rafael, J.A. | Limeira-de-Oliveira, F. | Takiya, D.M. | et al.; identifiedBy: Allan Paulo Moreira dos Santos; institutionCode: DZRJ; basisOfRecord: PreservedSpecimen

j. country: Brazil; stateProvince: Ceará; municipality: Ubajara; locality: Parque Nacional de Ubajara, Trilha Araticum, Rio das Minas na altura da trilha do teleférico; maximumElevationInMeters: 420; verbatimCoordinates: $3^{\circ} 49^{\prime} 58^{\prime \prime S}, 40^{\circ} 53^{\prime} 53 " \mathrm{~W}$; samplingProtocol: Malaise intercept trap; verbatimEventDate: 20.iv.12; individualCount: 1; sex: male; lifeStage: adult; recordedBy: Rafael, J.A. | Limeira-de-Oliveira, F. | Takiya, D.M. | et al.; identifiedBy: Allan Paulo Moreira dos Santos; institutionCode: DZRJ; basisOfRecord: PreservedSpecimen

k. country: Brazil; stateProvince: Ceará; municipality: Ubajara; locality: Parque Nacional de Ubajara, Trilha Araticum, Rio das Minas na altura da trilha do teleférico; maximumElevationInMeters: 420; verbatimCoordinates: $3^{\circ} 49^{\prime} 58^{\prime \prime S}, 40^{\circ} 53^{\prime} 53^{\prime \prime} \mathrm{W}$;

samplingProtocol: Malaise intercept trap; verbatimEventDate: 20.iv.12; individualCount: 2; sex: male; lifeStage: adult; recordedBy: Rafael, J.A. | Limeira-de-Oliveira, F. | Takiya, D.M. I et al.; identifiedBy: Allan Paulo Moreira dos Santos; institutionCode: DZRJ; basisOfRecord: PreservedSpecimen

I. country: Brazil; stateProvince: Ceará; municipality: Ubajara; locality: Parque Nacional de Ubajara, Trilha Araticum, Rio das Minas na altura da trilha do teleférico; maximumElevationInMeters: 420; verbatimCoordinates: $3^{\circ} 49^{\prime} 58^{\prime \prime S}, 40^{\circ} 53^{\prime} 53^{\prime \prime} \mathrm{W}$; samplingProtocol: Malaise intercept trap; verbatimEventDate: 20.iv.12; individualCount: 6; sex: male; lifeStage: adult; recordedBy: Rafael, J.A. | Limeira-de-Oliveira, F. | Takiya, D.M. | et al.; identifiedBy: Allan Paulo Moreira dos Santos; institutionCode: DZRJ; basisOfRecord: PreservedSpecimen

Notes: Undescribed species.

\section{Alisotrichia sp. 2}

\section{Materials}

a. country: Brazil; stateProvince: Ceará; municipality: Ubajara; locality: Parque Nacional de Ubajara, Rio das Minas, próximo ao Portão Araticum; maximumElevationInMeters: 328; verbatimCoordinates: $3^{\circ} 49^{\prime} 32.6^{\prime \prime S}, 40^{\circ} 53^{\prime} 32.8^{\prime \prime W}$; sampling Protocol: Malaise intercept trap; verbatimEventDate: 17.ii.13; individualCount: 1; sex: male; lifeStage: adult; recordedBy: Rafael, J.A. | Limeira-de-Oliveira, F. | Takiya, D.M. | Santos, A.P.M. | et al.; identifiedBy: Allan Paulo Moreira dos Santos; institutionCode: DZRJ; basisOfRecord: PreservedSpecimen

b. country: Brazil; stateProvince: Ceará; municipality: Ubajara; locality: Parque Nacional de Ubajara, Trilha Araticum, Rio das Minas na altura da trilha do teleférico; maximumElevationInMeters: 420; verbatimCoordinates: 349'58"S, 4053'53"W; samplingProtocol: Malaise intercept trap; verbatimEventDate: 20.iv.12; individualCount: 1; sex: male; lifeStage: adult; recordedBy: Rafael, J.A. | Limeira-de-Oliveira, F. | Takiya, D.M. I et al.; identifiedBy: Allan Paulo Moreira dos Santos; institutionCode: DZRJ; basisOfRecord: PreservedSpecimen 
c. country: Brazil; stateProvince: Ceará; municipality: Ubajara; locality: Parque Nacional de Ubajara, Trilha Araticum, Rio das Minas na altura da trilha do teleférico; maximumElevationInMeters: 420; verbatimCoordinates: $3^{\circ} 49^{\prime} 58^{\prime \prime S}, 40^{\circ} 53^{\prime} 53^{\prime \prime} \mathrm{W}$; samplingProtocol: Malaise intercept trap; verbatimEventDate: 20.iv.12; individualCount: 1; sex: female; lifeStage: adult; recordedBy: Rafael, J.A. | Limeira-de-Oliveira, F. | Takiya, D.M. | et al.; identifiedBy: Allan Paulo Moreira dos Santos; institutionCode: DZRJ; basisOfRecord: PreservedSpecimen

d. country: Brazil; stateProvince: Ceará; municipality: Ubajara; locality: Parque Nacional de Ubajara, Trilha Araticum, Rio das Minas na altura da trilha do teleférico; maximumElevationInMeters: 420; verbatimCoordinates: $3^{\circ} 49^{\prime} 58^{\prime \prime S}, 40^{\circ} 53^{\prime} 53 " \mathrm{~W}$; samplingProtocol: Malaise intercept trap; verbatimEventDate: 20.iv.12; individualCount: 11; sex: male; lifeStage: adult; recordedBy: Rafael, J.A. | Limeira-de-Oliveira, F. | Takiya, D.M. | et al.; identifiedBy: Allan Paulo Moreira dos Santos; institutionCode: DZRJ; basisOfRecord: PreservedSpecimen

e. $\quad$ country: Brazil; stateProvince: Ceará; municipality: Ubajara; locality: Parque Nacional de Ubajara, Trilha Araticum, Rio das Minas na altura da trilha do teleférico; maximumElevationInMeters: 420; verbatimCoordinates: $3^{\circ} 49^{\prime} 58^{\prime \prime S}, 40^{\circ} 53^{\prime} 53^{\prime \prime W}$; samplingProtocol: Malaise intercept trap; verbatimEventDate: 20.iv.12; individualCount: 18; sex: male; lifeStage: adult; recordedBy: Rafael, J.A. | Limeira-de-Oliveira, F. | Takiya, D.M. | et al.; identifiedBy: Allan Paulo Moreira dos Santos; institutionCode: DZRJ; basisOfRecord: PreservedSpecimen

f. country: Brazil; stateProvince: Ceará; municipality: Ubajara; locality: Parque Nacional de Ubajara, Trilha Araticum, Rio das Minas na altura da trilha do teleférico; maximumElevationInMeters: 420; verbatimCoordinates: $3^{\circ} 49^{\prime} 58^{\prime \prime S}, 40^{\circ} 53^{\prime} 53^{\prime \prime} \mathrm{W}$; samplingProtocol: Malaise intercept trap; verbatimEventDate: 20.iv.12; individualCount: 25; sex: male; lifeStage: adult; recordedBy: Rafael, J.A. | Limeira-de-Oliveira, F. | Takiya, D.M. | et al.; identifiedBy: Allan Paulo Moreira dos Santos; institutionCode: DZRJ; basisOfRecord: PreservedSpecimen

g. country: Brazil; stateProvince: Ceará; municipality: Ubajara; locality: Parque Nacional de Ubajara, Trilha Araticum, Rio das Minas na altura da trilha do teleférico; maximumElevationInMeters: 420; verbatimCoordinates: $3^{\circ} 49^{\prime} 58^{\prime \prime S}, 40^{\circ} 53^{\prime} 53 " \mathrm{~W}$; samplingProtocol: Malaise intercept trap; verbatimEventDate: $20 . i v .12$; individualCount: 27; sex: male; lifeStage: adult; recordedBy: Rafael, J.A. | Limeira-de-Oliveira, F. | Takiya, D.M. | et al.; identifiedBy: Allan Paulo Moreira dos Santos; institutionCode: DZRJ; basisOfRecord: PreservedSpecimen

h. country: Brazil; stateProvince: Ceará; municipality: Ubajara; locality: Parque Nacional de Ubajara, Trilha Araticum, Rio das Minas na altura da trilha do teleférico; maximumElevationInMeters: 420; verbatimCoordinates: $3^{\circ} 49^{\prime} 58^{\prime \prime S}, 40^{\circ} 53^{\prime} 53 " \mathrm{~W}$; samplingProtocol: Malaise intercept trap; verbatimEventDate: 20.iv.12; individualCount: 79; sex: male; lifeStage: adult; recordedBy: Rafael, J.A. | Limeira-de-Oliveira, F. | Takiya, D.M. | et al.; identifiedBy: Allan Paulo Moreira dos Santos; institutionCode: DZRJ; basisOfRecord: PreservedSpecimen

i. country: Brazil; stateProvince: Ceará; municipality: Ubajara; locality: Parque Nacional de Ubajara, Trilha Araticum, Rio das Minas na altura da trilha do teleférico; maximumElevationInMeters: 420; verbatimCoordinates: $3^{\circ} 49^{\prime} 58^{\prime \prime S}, 40^{\circ} 53^{\prime} 53$ "W; samplingProtocol: Malaise intercept trap; verbatimEventDate: 21.v.12; individualCount: 1; sex: male; lifeStage: adult; recordedBy: Limeira-de-Oliveira | et al.; identifiedBy: Allan Paulo Moreira dos Santos; institutionCode: DZRJ; basisOfRecord: PreservedSpecimen

j. $\quad$ country: Brazil; stateProvince: Ceará; municipality: Ubajara; locality: Parque Nacional de Ubajara, Trilha Araticum, Rio das Minas na altura da trilha do teleférico; 
maximumElevationInMeters: 420; verbatimCoordinates: $3^{\circ} 49^{\prime} 58$ "S, 4053'53"W; samplingProtocol: Malaise intercept trap; verbatimEventDate: 21.v.12; individualCount: 7; sex: female; lifeStage: adult; recordedBy: Limeira-de-Oliveira | et al.; identifiedBy: Allan Paulo Moreira dos Santos; institutionCode: DZRJ; basisOfRecord: PreservedSpecimen

k. country: Brazil; stateProvince: Ceará; municipality: Ubajara; locality: Parque Nacional de Ubajara, Trilha Araticum, Rio das Minas na altura da trilha do teleférico; maximumElevationInMeters: 420; verbatimCoordinates: 349'58"S, 4053'53"W; samplingProtocol: Malaise intercept trap; verbatimEventDate: 20.iv.12; individualCount: 2; sex: male; lifeStage: adult; recordedBy: Rafael, J.A. | Limeira-de-Oliveira, F. | Takiya, D.M. | et al.; identifiedBy: Allan Paulo Moreira dos Santos; institutionCode: DZRJ; basisOfRecord: PreservedSpecimen

I. country: Brazil; stateProvince: Ceará; municipality: Ubajara; locality: Parque Nacional de Ubajara, Trilha Araticum, Rio das Minas na altura da trilha do teleférico; maximumElevationInMeters: 420; verbatimCoordinates: $3^{\circ} 49^{\prime} 58^{\prime \prime S}, 40^{\circ} 53^{\prime} 53^{\prime \prime} \mathrm{W}$; samplingProtocol: Malaise intercept trap; verbatimEventDate: 20.iv.12; individualCount: 6; sex: male; lifeStage: adult; recordedBy: Rafael, J.A. | Limeira-de-Oliveira, F. | Takiya, D.M. | et al.; identifiedBy: Allan Paulo Moreira dos Santos; institutionCode: DZRJ; basisOfRecord: PreservedSpecimen

Notes: Undescribed species.

\section{Alisotrichia sp. 3}

\section{Materials}

a. country: Brazil; stateProvince: Ceará; municipality: Ubajara; locality: Parque Nacional de Ubajara, Trilha Araticum, Rio das Minas na altura da trilha do teleférico; maximumElevationInMeters: 420; verbatimCoordinates: $3^{\circ} 49^{\prime} 58^{\prime \prime S}, 40^{\circ} 53^{\prime} 53^{\prime \prime} \mathrm{W}$; samplingProtocol: Malaise intercept trap; verbatimEventDate: 20.iv.12; individualCount: 1; sex: male; lifeStage: adult; recordedBy: Rafael, J.A. | Limeira-de-Oliveira, F. | Takiya, D.M. I et al.; identifiedBy: Allan Paulo Moreira dos Santos; institutionCode: DZRJ; basisOfRecord: PreservedSpecimen

b. $\quad$ country: Brazil; stateProvince: Ceará; municipality: Ubajara; locality: Parque Nacional de Ubajara, Trilha Araticum, Rio das Minas na altura da trilha do teleférico; maximumElevationInMeters: 420; verbatimCoordinates: $3^{\circ} 49^{\prime} 58^{\prime \prime S}, 40^{\circ} 53^{\prime} 53^{\prime \prime} \mathrm{W}$; samplingProtocol: Malaise intercept trap; verbatimEventDate: 20.iv.12; individualCount: 1; sex: male; lifeStage: adult; recordedBy: Rafael, J.A. | Limeira-de-Oliveira, F. | Takiya, D.M. I et al.; identifiedBy: Allan Paulo Moreira dos Santos; institutionCode: DZRJ; basisOfRecord: PreservedSpecimen

c. country: Brazil; stateProvince: Ceará; municipality: Ubajara; locality: Parque Nacional de Ubajara, Trilha Araticum, Rio das Minas na altura da trilha do teleférico; maximumElevationInMeters: 420; verbatimCoordinates: $3^{\circ} 49^{\prime} 58^{\prime \prime S}, 40^{\circ} 53^{\prime} 53^{\prime \prime} \mathrm{W}$; samplingProtocol: Malaise intercept trap; verbatimEventDate: 20.iv.12; individualCount: 1; sex: female; lifeStage: adult; recordedBy: Rafael, J.A. | Limeira-de-Oliveira, F. | Takiya, D.M. | et al.; identifiedBy: Allan Paulo Moreira dos Santos; institutionCode: DZRJ; basisOfRecord: PreservedSpecimen

d. country: Brazil; stateProvince: Ceará; municipality: Ubajara; locality: Parque Nacional de Ubajara, Trilha Araticum, Rio das Minas na altura da trilha do teleférico; maximumElevationInMeters: 420; verbatimCoordinates: $3^{\circ} 49^{\prime} 58^{\prime \prime S}, 40^{\circ} 53^{\prime} 53^{\prime \prime} \mathrm{W}$; samplingProtocol: Malaise intercept trap; verbatimEventDate: 20.iv.12; individualCount: 
11; sex: male; lifeStage: adult; recordedBy: Rafael, J.A. | Limeira-de-Oliveira, F. | Takiya, D.M. | et al.; identifiedBy: Allan Paulo Moreira dos Santos; institutionCode: DZRJ; basisOfRecord: PreservedSpecimen

e. country: Brazil; stateProvince: Ceará; municipality: Ubajara; locality: Parque Nacional de Ubajara, Trilha Araticum, Rio das Minas na altura da trilha do teleférico;

maximumElevationInMeters: 420; verbatimCoordinates: $3^{\circ} 49^{\prime} 58^{\prime \prime S}, 40^{\circ} 53^{\prime} 53^{\prime \prime} \mathrm{W}$;

samplingProtocol: Malaise intercept trap; verbatimEventDate: 20.iv.12; individualCount:

18; sex: male; lifeStage: adult; recordedBy: Rafael, J.A. | Limeira-de-Oliveira, F. | Takiya,

D.M. | et al.; identifiedBy: Allan Paulo Moreira dos Santos; institutionCode: DZRJ; basisOfRecord: PreservedSpecimen

f. country: Brazil; stateProvince: Ceará; municipality: Ubajara; locality: Parque Nacional de Ubajara, Trilha Araticum, Rio das Minas na altura da trilha do teleférico;

maximumElevationInMeters: 420; verbatimCoordinates: $3^{\circ} 49^{\prime} 58^{\prime \prime S}, 40^{\circ} 53^{\prime} 53^{\prime \prime} \mathrm{W}$;

samplingProtocol: Malaise intercept trap; verbatimEventDate: 20.iv.12; individualCount:

25; sex: male; lifeStage: adult; recordedBy: Rafael, J.A. | Limeira-de-Oliveira, F. | Takiya,

D.M. | et al.; identifiedBy: Allan Paulo Moreira dos Santos; institutionCode: DZRJ;

basisOfRecord: PreservedSpecimen

g. country: Brazil; stateProvince: Ceará; municipality: Ubajara; locality: Parque Nacional de Ubajara, Trilha Araticum, Rio das Minas na altura da trilha do teleférico;

maximumElevationInMeters: 420; verbatimCoordinates: $3^{\circ} 49^{\prime} 58$ "S, 4053'53"W;

samplingProtocol: Malaise intercept trap; verbatimEventDate: $20 . i v .12$; individualCount:

27; sex: male; lifeStage: adult; recordedBy: Rafael, J.A. | Limeira-de-Oliveira, F. | Takiya,

D.M. | et al.; identifiedBy: Allan Paulo Moreira dos Santos; institutionCode: DZRJ;

basisOfRecord: PreservedSpecimen

h. country: Brazil; stateProvince: Ceará; municipality: Ubajara; locality: Parque Nacional de Ubajara, Trilha Araticum, Rio das Minas na altura da trilha do teleférico;

maximumElevationInMeters: 420; verbatimCoordinates: $3^{\circ} 49^{\prime} 58^{\prime \prime S}, 40^{\circ} 53^{\prime} 53^{\prime \prime} \mathrm{W}$;

samplingProtocol: Malaise intercept trap; verbatimEventDate: 20.iv.12; individualCount:

79; sex: male; lifeStage: adult; recordedBy: Rafael, J.A. | Limeira-de-Oliveira, F. | Takiya,

D.M. | et al.; identifiedBy: Allan Paulo Moreira dos Santos; institutionCode: DZRJ; basisOfRecord: PreservedSpecimen

i. country: Brazil; stateProvince: Ceará; municipality: Ubajara; locality: Parque Nacional de Ubajara, Trilha Araticum, Rio das Minas na altura da trilha do teleférico;

maximumElevationInMeters: 420; verbatimCoordinates: $3^{\circ} 49^{\prime} 58$ "S, 4053'53"W;

samplingProtocol: Malaise intercept trap; verbatimEventDate: 21.v.12; individualCount: 1; sex: male; lifeStage: adult; recordedBy: Limeira-de-Oliveira | et al.; identifiedBy: Allan Paulo Moreira dos Santos; institutionCode: DZRJ; basisOfRecord: PreservedSpecimen

j. country: Brazil; stateProvince: Ceará; municipality: Ubajara; locality: Parque Nacional de Ubajara, Trilha Araticum, Rio das Minas na altura da trilha do teleférico; maximumElevationInMeters: 420; verbatimCoordinates: $3^{\circ} 49^{\prime} 58^{\prime \prime S}, 40^{\circ} 53^{\prime} 53 " \mathrm{~W}$; samplingProtocol: Malaise intercept trap; verbatimEventDate: 21.v.12; individualCount: 7; sex: female; lifeStage: adult; recordedBy: Limeira-de-Oliveira | et al.; identifiedBy: Allan Paulo Moreira dos Santos; institutionCode: DZRJ; basisOfRecord: PreservedSpecimen

Notes: Undescribed species. 


\section{Genus Hydroptila Dalman, 1918}

Notes: Genus firstly record from CE in Souza et al. 2014a.

\section{Hydroptila marighellai Souza, Santos \& Takiya, 2014}

\section{Materials}

a. $\quad$ country: Brazil; stateProvince: Ceará; municipality: Ubajara; locality: Parque Nacional de Ubajara, Trilha Araticum, Rio das Minas na altura da trilha do teleférico; maximumElevationInMeters: 420; verbatimCoordinates: $3^{\circ} 49^{\prime} 58$ "S, 4053'53"W; samplingProtocol: Malaise intercept trap; verbatimEventDate: 20.iv.12; individualCount: 2; sex: male; lifeStage: adult; recordedBy: Rafael, J.A. | Limeira-de-Oliveira, F. | Takiya, D.M. | et al.; identifiedBy: Allan Paulo Moreira dos Santos; institutionCode: DZRJ; basisOfRecord: PreservedSpecimen

b. $\quad$ country: Brazil; stateProvince: Ceará; municipality: Ubajara; locality: Parque Nacional de Ubajara, Trilha Araticum, Rio das Minas na altura da trilha do teleférico; maximumElevationInMeters: 420; verbatimCoordinates: $3^{\circ} 49^{\prime} 58^{\prime \prime S}$, 4053'53"W; samplingProtocol: Malaise intercept trap; verbatimEventDate: 20.iv.12; individualCount: 3; sex: female; lifeStage: adult; recordedBy: Rafael, J.A. | Limeira-de-Oliveira, F. | Takiya, D.M. | et al.; identifiedBy: Allan Paulo Moreira dos Santos; institutionCode: DZRJ; basisOfRecord: PreservedSpecimen

c. country: Brazil; stateProvince: Ceará; municipality: Ubajara; locality: Parque Nacional de Ubajara, Trilha Araticum, Rio das Minas; maximumElevationInMeters: 524; verbatimCoordinates: $3^{\circ} 50^{\prime} 3$ "S, $40^{\circ} 54^{\prime} 18 \mathrm{~W}$; samplingProtocol: Pennsylvania light trap; verbatimEventDate: 22.iv.12; individualCount: 2; sex: male; lifeStage: adult; recordedBy: Takiya, D.M. | Somavilla, A.; identifiedBy: Allan Paulo Moreira dos Santos; institutionCode: DZRJ; basisOfRecord: PreservedSpecimen

Distribution: Brazil: CE, PE, AL.

Notes: Species described in Souza et al. 2014a.

\section{Genus Metrichia Ross, 1938}

Notes: Genus firstly recorded from CE in Santos et al. 2016a.

\section{Metrichia acuminata Santos, Takiya \& Nessimian, 2016}

\section{Materials}

a. country: Brazil; stateProvince: Ceará; municipality: Ubajara; locality: Parque Nacional de Ubajara, Rio Cafundó, pouco acima da cachoeira; maximumElevationInMeters: 795; verbatimCoordinates: $3^{\circ} 50^{\prime} 13^{\prime \prime} \mathrm{S}, 40^{\circ} 54^{\prime} 35 \mathrm{WW}$; samplingProtocol: Malaise intercept trap; verbatimEventDate: 13.xi.12; individualCount: 2; sex: male; lifeStage: adult; recordedBy: Limeira-de-Oliveira | et al.; identifiedBy: Allan Paulo Moreira dos Santos; institutionCode: DZRJ; basisOfRecord: PreservedSpecimen

b. country: Brazil; stateProvince: Ceará; municipality: Ubajara; locality: Parque Nacional de Ubajara, Trilha Araticum, Rio das Minas; maximumElevationInMeters: 524; verbatimCoordinates: $3^{\circ} 50^{\prime} 3$ "S, $40^{\circ} 54^{\prime} 18^{\prime \prime} \mathrm{W}$; samplingProtocol: Malaise intercept trap; 
verbatimEventDate: 14.ii.13; individualCount: 2; sex: male; lifeStage: adult; recordedBy: Rafael, J.A. | Limeira-de-Oliveira, F. | Takiya, D.M. | Santos, A.P.M. | et al.; identifiedBy: Allan Paulo Moreira dos Santos; institutionCode: DZRJ; basisOfRecord: PreservedSpecimen

c. country: Brazil; stateProvince: Ceará; municipality: Ubajara; locality: Parque Nacional de Ubajara, Trilha Samambaia, Mirante da cachoeira do Gameleira; maximumElevationInMeters: 880; verbatimCoordinates: $3^{\circ} 50^{\prime} 21$ 'S, 4054'23"W; samplingProtocol: Pennsylvania light trap; verbatimEventDate: 23.iv.12; individualCount: 1; sex: male; lifeStage: adult; recordedBy: Takiya, D.M. | Câmara, J.T.; identifiedBy: Allan Paulo Moreira dos Santos; institutionCode: DZRJ; basisOfRecord: PreservedSpecimen

Distribution: Brazil: CE, AL.

Notes: Species described in Santos et al. 2016a.

\section{Metrichia vulgaris Santos, Takiya \& Nessimian, 2016}

\section{Materials}

a. country: Brazil; stateProvince: Ceará; municipality: Ubajara; locality: Parque Nacional de Ubajara, Trilha Samambaia, Rio Gameleira; maximumElevationInMeters: 874; verbatimCoordinates: $3^{\circ} 50^{\prime} 25^{\prime \prime} \mathrm{S}, 40^{\circ} 54^{\prime} 19^{\prime \prime W}$; samplingProtocol: Malaise intercept trap; verbatimEventDate: 20.iv.12; individualCount: 1; sex: male; lifeStage: adult; recordedBy: Rafael, J.A. | Limeira-de-Oliveira, F. | Takiya, D.M. | et al.; identifiedBy: Allan Paulo Moreira dos Santos; institutionCode: DZRJ; basisOfRecord: PreservedSpecimen

b. country: Brazil; stateProvince: Ceará; municipality: Ubajara; locality: Parque Nacional de Ubajara, Trilha Araticum, Rio das Minas; maximumElevationInMeters: 524; verbatimCoordinates: $3^{\circ} 50^{\prime} 3$ "S, $40^{\circ} 54^{\prime} 18^{\prime \prime} \mathrm{W}$; samplingProtocol: Malaise intercept trap; verbatimEventDate: 14.ii.13; individualCount: 2; sex: male; lifeStage: adult; recordedBy: Rafael, J.A. | Limeira-de-Oliveira, F. | Takiya, D.M. | Santos, A.P.M. | et al.; identifiedBy: Allan Paulo Moreira dos Santos; institutionCode: DZRJ; basisOfRecord: PreservedSpecimen

c. country: Brazil; stateProvince: Ceará; municipality: Ubajara; locality: Parque Nacional de Ubajara, Trilha Samambaia, Mirante da cachoeira do Gameleira; maximumElevationInMeters: 880; verbatimCoordinates: $3^{\circ} 50^{\prime} 21$ "S, 4054'23"W; samplingProtocol: Pennsylvania light trap; verbatimEventDate: 23.iv.12; individualCount: 1; sex: male; lifeStage: adult; recordedBy: Takiya, D.M. | Câmara, J.T.; identifiedBy: Allan Paulo Moreira dos Santos; institutionCode: DZRJ; basisOfRecord: PreservedSpecimen

Distribution: Brazil: CE, GO, MG, RJ.

Notes: Species described in Santos et al. 2016a.

\section{Metrichia rafaeli Santos, Takiya \& Nessimian, 2016}

\section{Materials}

a. $\quad$ country: Brazil; stateProvince: Ceará; municipality: Ubajara; locality: Parque Nacional de Ubajara, Rio Cafundó, pouco acima da cachoeira; maximumElevationInMeters: 795; verbatimCoordinates: $3^{\circ} 50^{\prime} 13^{\prime \prime S}, 40^{\circ} 54^{\prime} 35 " \mathrm{~W}$; samplingProtocol: Malaise intercept trap; 
verbatimEventDate: 13.ix.12; individualCount: 1; sex: male; lifeStage: adult; recordedBy: Limeira-de-Oliveira | et al.; identifiedBy: Allan Paulo Moreira dos Santos; institutionCode: DZRJ; basisOfRecord: PreservedSpecimen

b. $\quad$ country: Brazil; stateProvince: Ceará; municipality: Ubajara; locality: Parque Nacional de Ubajara, Trilha Araticum, Rio das Minas na altura da trilha do teleférico; maximumElevationInMeters: 420; verbatimCoordinates: $3^{\circ} 49^{\prime} 58^{\prime \prime S}, 40^{\circ} 53^{\prime} 53^{\prime \prime} \mathrm{W}$; samplingProtocol: Malaise intercept trap; verbatimEventDate: 14.ii.13; individualCount: 1; sex: male; lifeStage: adult; recordedBy: Rafael, J.A. | Limeira-de-Oliveira, F. | Takiya, D.M. | Santos, A.P.M. | et al.; identifiedBy: Allan Paulo Moreira dos Santos; institutionCode: DZRJ; basisOfRecord: PreservedSpecimen

c. country: Brazil; stateProvince: Ceará; municipality: Ubajara; locality: Parque Nacional de Ubajara, Cachoeira do Cafundó; maximumElevationInMeters: 783; verbatimCoordinates: $3^{\circ} 50^{\prime} 12$ "S, 4054'35"W; samplingProtocol: Pennsylvania light trap; verbatimEventDate: 15.ii.13; individualCount: 1; sex: male; lifeStage: adult; recordedBy: Santos, A.P.M. | Takiya, D.M.; identifiedBy: Allan Paulo Moreira dos Santos; institutionCode: DZRJ; basisOfRecord: PreservedSpecimen

d. country: Brazil; stateProvince: Ceará; municipality: Ubajara; locality: Parque Nacional de Ubajara, Trilha Samambaia, Rio Gameleira; maximumElevationInMeters: 874; verbatimCoordinates: $3^{\circ} 50^{\prime 2} 2$ "S, $^{\circ} 0^{\circ} 54^{\prime} 19 \mathrm{\prime W}$; samplingProtocol: Malaise intercept trap; verbatimEventDate: 17.ii.13; individualCount: 1; sex: male; lifeStage: adult; recordedBy: Rafael, J.A. | Limeira-de-Oliveira, F. | Takiya, D.M. | Santos, A.P.M. | et al.; identifiedBy: Allan Paulo Moreira dos Santos; institutionCode: DZRJ; basisOfRecord: PreservedSpecimen

e. country: Brazil; stateProvince: Ceará; municipality: Ubajara; locality: Parque Nacional de Ubajara, Trilha Araticum, Rio das Minas; maximumElevationInMeters: 524; verbatimCoordinates: $3^{\circ} 50^{\prime} 3$ "S, 4054'18"W; samplingProtocol: Malaise intercept trap; verbatimEventDate: 17.ii.13; individualCount: 2; sex: male; lifeStage: adult; recordedBy: Rafael, J.A. | Limeira-de-Oliveira, F. | Takiya, D.M. | Santos, A.P.M. | et al.; identifiedBy: Allan Paulo Moreira dos Santos; institutionCode: DZRJ; basisOfRecord: PreservedSpecimen

f. country: Brazil; stateProvince: Ceará; municipality: Ubajara; locality: Parque Nacional de Ubajara, Trilha Araticum, Rio das Minas na altura da trilha do teleférico; maximumElevationInMeters: 420; verbatimCoordinates: $3^{\circ} 49^{\prime} 58^{\prime \prime S}$, 4053'53"W; samplingProtocol: Malaise intercept trap; verbatimEventDate: 20.iv.12; individualCount: 1; sex: male; lifeStage: adult; recordedBy: Rafael, J.A. | Limeira-de-Oliveira, F. | Takiya, D.M. | et al.; identifiedBy: Allan Paulo Moreira dos Santos; institutionCode: DZRJ; basisOfRecord: PreservedSpecimen

Distribution: Brazil: CE.

Notes: Species described in Santos et al. 2016a.

\section{Metrichia ubajara Santos, Takiya \& Nessimian, 2016}

\section{Materials}

a. country: Brazil; stateProvince: Ceará; municipality: Ubajara; locality: Parque Nacional de Ubajara, Rio Cafundó, pouco acima da cachoeira; maximumElevationInMeters: 795; verbatimCoordinates: $3^{\circ} 50^{\prime} 13^{\prime \prime} \mathrm{S}, 40^{\circ} 54^{\prime} 35 \mathrm{WW}$; samplingProtocol: Malaise intercept trap; verbatimEventDate: 13.xi.12; individualCount: 2; sex: male; lifeStage: adult; recordedBy: 
Limeira-de-Oliveira | et al.; identifiedBy: Allan Paulo Moreira dos Santos; institutionCode: DZRJ; basisOfRecord: PreservedSpecimen

b. country: Brazil; stateProvince: Ceará; municipality: Ubajara; locality: Parque Nacional de Ubajara, Rio Cafundó, pouco acima da cachoeira; maximumElevationInMeters: 795; verbatimCoordinates: $3^{\circ} 50^{\prime} 13^{\prime \prime} \mathrm{S}, 40^{\circ} 54^{\prime} 35 \mathrm{~W} \mathrm{~W}$; samplingProtocol: Malaise intercept trap; verbatimEventDate: 13.xi.12; individualCount: 10; sex: male; lifeStage: adult; recordedBy: Limeira-de-Oliveira | et al.; identifiedBy: Allan Paulo Moreira dos Santos; institutionCode: DZRJ; basisOfRecord: PreservedSpecimen

c. country: Brazil; stateProvince: Ceará; municipality: Ubajara; locality: Parque Nacional de Ubajara, Rio Cafundó, pouco acima da cachoeira; maximumElevationInMeters: 795; verbatimCoordinates: $3^{\circ} 50^{\prime} 13^{\prime \prime} \mathrm{S}, 40^{\circ} 54^{\prime} 35 " \mathrm{~W}$; samplingProtocol: Malaise intercept trap; verbatimEventDate: 13.xi.12; individualCount: 26; sex: male; lifeStage: adult; recordedBy: Limeira-de-Oliveira | et al.; identifiedBy: Allan Paulo Moreira dos Santos; institutionCode: DZRJ; basisOfRecord: PreservedSpecimen

d. country: Brazil; stateProvince: Ceará; municipality: Ubajara; locality: Parque Nacional de Ubajara, Trilha Araticum, Rio das Minas; maximumElevationInMeters: 524; verbatimCoordinates: $3^{\circ} 50^{\prime} 3$ "S, $40^{\circ} 54^{\prime} 18$ "W; samplingProtocol: Malaise intercept trap; verbatimEventDate: 14.ii.13; individualCount: 7; sex: male; lifeStage: adult; recordedBy: Rafael, J.A. | Limeira-de-Oliveira, F. | Takiya, D.M. | Santos, A.P.M. | et al.; identifiedBy: Allan Paulo Moreira dos Santos; institutionCode: DZRJ; basisOfRecord: PreservedSpecimen

e. $\quad$ country: Brazil; stateProvince: Ceará; municipality: Ubajara; locality: Parque Nacional de Ubajara, Trilha Araticum, Rio das Minas na altura da trilha do teleférico; maximumElevationInMeters: 420; verbatimCoordinates: $3^{\circ} 49^{\prime} 58^{\prime \prime} \mathrm{S}, 40^{\circ} 53^{\prime} 53^{\prime \prime} \mathrm{W}$; samplingProtocol: Malaise intercept trap; verbatimEventDate: 14.ii.13; individualCount: 9; sex: male; lifeStage: adult; recordedBy: Rafael, J.A. | Limeira-de-Oliveira, F. | Takiya, D.M. | Santos, A.P.M. | et al.; identifiedBy: Allan Paulo Moreira dos Santos; institutionCode: DZRJ; basisOfRecord: PreservedSpecimen

f. country: Brazil; stateProvince: Ceará; municipality: Ubajara; locality: Parque Nacional de Ubajara, Rio das Minas, próximo ao Portão Araticum; maximumElevationInMeters: 328; verbatimCoordinates: $3^{\circ} 49^{\prime} 32.6^{\prime \prime S}, 40^{\circ} 53^{\prime} 32.8^{\prime \prime} \mathrm{W}$; samplingProtocol: Malaise intercept trap; verbatimEventDate: 14.ii.13; individualCount: 2; sex: male; lifeStage: adult; recordedBy: Rafael, J.A. | Limeira-de-Oliveira, F. | Takiya, D.M. | Santos, A.P.M. | et al.; identifiedBy: Allan Paulo Moreira dos Santos; institutionCode: DZRJ; basisOfRecord: PreservedSpecimen

g. country: Brazil; stateProvince: Ceará; municipality: Ubajara; locality: Parque Nacional de Ubajara, Rio Cafundó, pouco acima da cachoeira; maximumElevationInMeters: 795; verbatimCoordinates: $3^{\circ} 50^{\prime} 13^{\prime \prime S}, 40^{\circ} 54^{\prime} 35 " \mathrm{~W}$; samplingProtocol: Malaise intercept trap; verbatimEventDate: 18.xi.12; individualCount: 9; sex: male; lifeStage: adult; recordedBy: Limeira-de-Oliveira | et al.; identifiedBy: Allan Paulo Moreira dos Santos; institutionCode: DZRJ; basisOfRecord: PreservedSpecimen

h. country: Brazil; stateProvince: Ceará; municipality: Ubajara; locality: Parque Nacional de Ubajara, Trilha Araticum, Rio das Minas na altura da trilha do teleférico; maximumElevationInMeters: 420; verbatimCoordinates: $3^{\circ} 49^{\prime} 58^{\prime \prime S}$, 4053'53"W; samplingProtocol: Malaise intercept trap; verbatimEventDate: 20.iv.12; individualCount: 1; sex: male; lifeStage: adult; recordedBy: Rafael, J.A. | Limeira-de-Oliveira, F. | Takiya, D.M. I et al.; identifiedBy: Allan Paulo Moreira dos Santos; institutionCode: DZRJ; basisOfRecord: PreservedSpecimen

i. country: Brazil; stateProvince: Ceará; municipality: Ubajara; locality: Parque Nacional de Ubajara, Trilha Araticum, Rio das Minas na altura da trilha do teleférico; 
maximumElevationInMeters: 420 ; verbatimCoordinates: $3^{\circ} 49^{\prime} 58^{\prime \prime S}, 40^{\circ} 53^{\prime} 53^{\prime \prime} \mathrm{W}$;

samplingProtocol: Malaise intercept trap; verbatimEventDate: 20.iv.12; individualCount: 1; sex: male; lifeStage: adult; recordedBy: Rafael, J.A. | Limeira-de-Oliveira, F. | Takiya, D.M. | et al.; identifiedBy: Allan Paulo Moreira dos Santos; institutionCode: DZRJ; basisOfRecord: PreservedSpecimen

j. country: Brazil; stateProvince: Ceará; municipality: Ubajara; locality: Parque Nacional de Ubajara, Trilha Araticum, Rio das Minas na altura da trilha do teleférico; maximumElevationInMeters: 420; verbatimCoordinates: $3^{\circ} 49^{\prime} 58^{\prime \prime S}, 40^{\circ} 53^{\prime} 53 " \mathrm{~W}$;

samplingProtocol: Malaise intercept trap; verbatimEventDate: 20.iv.12; individualCount: 1; sex: male; lifeStage: adult; recordedBy: Rafael, J.A. | Limeira-de-Oliveira, F. | Takiya, D.M. | et al.; identifiedBy: Allan Paulo Moreira dos Santos; institutionCode: DZRJ; basisOfRecord: PreservedSpecimen

Distribution: Brazil: CE.

Notes: Species described in Santos et al. 2016a.

\section{Genus Neotrichia Morton, 1905}

Notes: New genus record for CE.

\section{Neotrichia sp. $2^{*}$}

\section{Materials}

a. country: Brazil; stateProvince: Ceará; municipality: Ubajara; locality: Parque Nacional de Ubajara, Trilha Samambaia, Rio Gameleira; maximumElevationInMeters: 874; verbatimCoordinates: $3^{\circ} 50^{\prime} 25 " \mathrm{~S}, 40^{\circ} 54^{\prime} 19 " \mathrm{~W}$; samplingProtocol: Pennsylvania light trap; verbatimEventDate: 13.ii.13; individualCount: 1; sex: male; lifeStage: adult; recordedBy: Santos, A.P.M. | Takiya, D.M.; identifiedBy: Allan Paulo Moreira dos Santos; institutionCode: DZRJ; basisOfRecord: PreservedSpecimen

b. country: Brazil; stateProvince: Ceará; municipality: Ubajara; locality: Parque Nacional de Ubajara, Trilha Araticum, Rio da Minas abaixo do teleférico; maximumElevationInMeters: 395; verbatimCoordinates: $3^{\circ} 49^{\prime} 43.3^{\prime \prime} \mathrm{S}, 40^{\circ} 53^{\prime} 51.5^{\prime \prime} \mathrm{W}$; samplingProtocol: Pennsylvania light trap; verbatimEventDate: 14.ii.13; individualCount: 1; sex: male; lifeStage: adult; recordedBy: Santos, A.P.M. | Takiya, D.M.; identifiedBy: Allan Paulo Moreira dos Santos; institutionCode: DZRJ; basisOfRecord: PreservedSpecimen

c. country: Brazil; stateProvince: Ceará; municipality: Ubajara; locality: Parque Nacional de Ubajara, Trilha Araticum, Rio das Minas na altura da trilha do teleférico; maximumElevationInMeters: 420; verbatimCoordinates: $3^{\circ} 49^{\prime} 58^{\prime \prime S}, 40^{\circ} 53^{\prime} 53 " \mathrm{~W}$; samplingProtocol: Malaise intercept trap; verbatimEventDate: 20.iv.12; individualCount: 1; sex: male; lifeStage: adult; recordedBy: Rafael, J.A. | Limeira-de-Oliveira, F. | Takiya, D.M. | et al.; identifiedBy: Allan Paulo Moreira dos Santos; institutionCode: DZRJ; basisOfRecord: PreservedSpecimen 


\section{Neotrichia sp. 5}

\section{Materials}

a. country: Brazil; stateProvince: Ceará; municipality: Ubajara; locality: Parque Nacional de Ubajara, Trilha Araticum, Rio das Minas na altura da trilha do teleférico; maximumElevationInMeters: 420 ; verbatimCoordinates: $3^{\circ} 49^{\prime} 58^{\prime \prime S}, 40^{\circ} 53^{\prime} 53^{\prime \prime} \mathrm{W}$; samplingProtocol: Malaise intercept trap; verbatimEventDate: 14.ii.13; individualCount: 1; sex: male; lifeStage: adult; recordedBy: Rafael, J.A. | Limeira-de-Oliveira, F. | Takiya, D.M. | Santos, A.P.M. | et al.; identifiedBy: Allan Paulo Moreira dos Santos; institutionCode: DZRJ; basisOfRecord: PreservedSpecimen

b. country: Brazil; stateProvince: Ceará; municipality: Ubajara; locality: Parque Nacional de Ubajara, Trilha Araticum, Rio da Minas abaixo do teleférico; maximumElevationInMeters: 395; verbatimCoordinates: $3^{\circ} 49^{\prime} 43.3^{\prime \prime S}$, 4053'51.5"W; samplingProtocol: Pennsylvania light trap; verbatimEventDate: 14.ii.13; individualCount: 1; sex: male; lifeStage: adult; recordedBy: Santos, A.P.M. | Takiya, D.M.; identifiedBy: Allan Paulo Moreira dos Santos; institutionCode: DZRJ; basisOfRecord: PreservedSpecimen

c. country: Brazil; stateProvince: Ceará; municipality: Ubajara; locality: Parque Nacional de Ubajara, Trilha Araticum, Rio das Minas na altura da trilha do teleférico; maximumElevationInMeters: 420; verbatimCoordinates: $3^{\circ} 49^{\prime} 58^{\prime \prime S}, 40^{\circ} 53^{\prime} 53^{\prime \prime} \mathrm{W}$; samplingProtocol: Malaise intercept trap; verbatimEventDate: 20.iv.12; individualCount: 1; sex: male; lifeStage: adult; recordedBy: Rafael, J.A. | Limeira-de-Oliveira, F. | Takiya, D.M. | et al.; identifiedBy: Allan Paulo Moreira dos Santos; institutionCode: DZRJ; basisOfRecord: PreservedSpecimen

\section{Genus Ochrotrichia Mosely, 1934}

Notes: Genus firstly recorded from CE in Souza et al. 2014a.

\section{Ochrotrichia caatinga Souza, Santos \& Takiya, 2014}

\section{Materials}

a. country: Brazil; stateProvince: Ceará; municipality: Ubajara; locality: Parque Nacional de Ubajara, Trilha Araticum, Rio das Minas; maximumElevationInMeters: 524; verbatimCoordinates: $3^{\circ} 50^{\prime} 3$ "S, $40^{\circ} 54^{\prime} 18^{\prime \prime W}$; sampling Protocol: Malaise intercept trap; verbatimEventDate: 14.ii.13; individualCount: 1; sex: male; lifeStage: adult; recordedBy: Rafael, J.A. | Limeira-de-Oliveira, F. | Takiya, D.M. | Santos, A.P.M. | et al.; identifiedBy: Allan Paulo Moreira dos Santos; institutionCode: DZRJ; basisOfRecord: PreservedSpecimen

b. $\quad$ country: Brazil; stateProvince: Ceará; municipality: Ubajara; locality: Parque Nacional de Ubajara, Trilha Samambaia, Rio Gameleira; maximumElevationInMeters: 874; verbatimCoordinates: $3^{\circ} 50^{\prime} 25^{\prime \prime} \mathrm{S}, 40^{\circ} 54^{\prime} 19^{\prime \prime W}$; samplingProtocol: Malaise intercept trap; verbatimEventDate: 17.ii.13; individualCount: 1; sex: male; lifeStage: adult; recordedBy: Rafael, J.A. | Limeira-de-Oliveira, F. | Takiya, D.M. | Santos, A.P.M. | et al.; identifiedBy: Allan Paulo Moreira dos Santos; institutionCode: DZRJ; basisOfRecord: PreservedSpecimen

c. country: Brazil; stateProvince: Ceará; municipality: Ubajara; locality: Parque Nacional de Ubajara, Trilha Araticum, Rio das Minas; maximumElevationInMeters: 524; verbatimCoordinates: $3^{\circ} 50^{\prime} 3^{\prime \prime} \mathrm{S}, 40^{\circ} 54^{\prime} 18^{\prime \prime} \mathrm{W}$; samplingProtocol: Malaise intercept trap; 
verbatimEventDate: 17.ii.13; individualCount: 1; sex: male; lifeStage: adult; recordedBy: Rafael, J.A. | Limeira-de-Oliveira, F. | Takiya, D.M. | Santos, A.P.M. | et al.; identifiedBy: Allan Paulo Moreira dos Santos; institutionCode: DZRJ; basisOfRecord: PreservedSpecimen

d. country: Brazil; stateProvince: Ceará; municipality: Ubajara; locality: Parque Nacional de Ubajara, Trilha Samambaia, Rio Gameleira; maximumElevationInMeters: 874; verbatimCoordinates: $3^{\circ} 50^{\prime 2} 25^{\prime} \mathrm{S}, 40^{\circ} 54^{\prime} 19^{\prime \prime W}$; samplingProtocol: Malaise intercept trap; verbatimEventDate: 20.iv.12; individualCount: 3; sex: male; lifeStage: adult; recordedBy: Rafael, J.A. | Limeira-de-Oliveira, F. | Takiya, D.M. | et al.; identifiedBy: Allan Paulo Moreira dos Santos; institutionCode: DZRJ; basisOfRecord: PreservedSpecimen

e. $\quad$ country: Brazil; stateProvince: Ceará; municipality: Ubajara; locality: Parque Nacional de Ubajara, Rio Cafundó, pouco acima da cachoeira; maximumElevationInMeters: 795; verbatimCoordinates: $3^{\circ} 50^{\prime} 13^{\prime \prime} \mathrm{S}, 40^{\circ} 54^{\prime} 35^{\prime \prime} \mathrm{W}$; samplingProtocol: Malaise intercept trap; verbatimEventDate: 21.iv.12; individualCount: 1; sex: male; lifeStage: adult; recordedBy: Rafael, J.A. | Limeira-de-Oliveira, F. | Takiya, D.M. | et al.; identifiedBy: Allan Paulo Moreira dos Santos; institutionCode: DZRJ; basisOfRecord: PreservedSpecimen

Distribution: Brazil: CE.

Notes: Species described in Souza et al. 2014a.

\section{Ochrotrichia limeirai Souza, Santos \& Takiya, 2014}

\section{Materials}

a. country: Brazil; stateProvince: Ceará; municipality: Ubajara; locality: Parque Nacional de Ubajara, Rio Cafundó, pouco acima da cachoeira; maximumElevationInMeters: 795; verbatimCoordinates: $3^{\circ} 50^{\prime} 13^{\prime \prime} \mathrm{S}, 40^{\circ} 54^{\prime} 35 \mathrm{WW}$; samplingProtocol: Malaise intercept trap; verbatimEventDate: 13.xi.12; individualCount: 1; sex: male; lifeStage: adult; recordedBy: Limeira-de-Oliveira | et al.; identifiedBy: Allan Paulo Moreira dos Santos; institutionCode: DZRJ; basisOfRecord: PreservedSpecimen

b. country: Brazil; stateProvince: Ceará; municipality: Ubajara; locality: Parque Nacional de Ubajara, Trilha Samambaia, Rio Gameleira; maximumElevationInMeters: 874; verbatimCoordinates: $3^{\circ} 50^{\prime} 25$ "S, 4054'19"W; samplingProtocol: Malaise intercept trap; verbatimEventDate: 17.ii.13; individualCount: 1; sex: male; lifeStage: adult; recordedBy: Rafael, J.A. | Limeira-de-Oliveira, F. | Takiya, D.M. | Santos, A.P.M. | et al.; identifiedBy: Allan Paulo Moreira dos Santos; institutionCode: DZRJ; basisOfRecord: PreservedSpecimen

c. country: Brazil; stateProvince: Ceará; municipality: Ubajara; locality: Parque Nacional de Ubajara, Trilha Araticum, Rio das Minas; maximumElevationInMeters: 524; verbatimCoordinates: $3^{\circ} 50^{\prime} 3$ "S, $40^{\circ} 54^{\prime} 18 \mathrm{~W}$; samplingProtocol: Malaise intercept trap; verbatimEventDate: 17.ii.13; individualCount: 1; sex: male; lifeStage: adult; recordedBy: Rafael, J.A. | Limeira-de-Oliveira, F. | Takiya, D.M. | Santos, A.P.M. | et al.; identifiedBy: Allan Paulo Moreira dos Santos; institutionCode: DZRJ; basisOfRecord: PreservedSpecimen

d. country: Brazil; stateProvince: Ceará; municipality: Ubajara; locality: Parque Nacional de Ubajara, Trilha Samambaia, Rio Gameleira; maximumElevationInMeters: 874; verbatimCoordinates: $3^{\circ} 50^{\prime} 25 " \mathrm{~S}, 40^{\circ} 54^{\prime} 19 \mathrm{~W} \mathrm{~W}$; samplingProtocol: Malaise intercept trap; verbatimEventDate: 20.iv.12; individualCount: 3; sex: male; lifeStage: adult; recordedBy: 
Rafael, J.A. | Limeira-de-Oliveira, F. | Takiya, D.M. | et al.; identifiedBy: Allan Paulo Moreira dos Santos; institutionCode: DZRJ; basisOfRecord: PreservedSpecimen

e. $\quad$ country: Brazil; stateProvince: Ceará; municipality: Ubajara; locality: Parque Nacional de Ubajara, Rio Cafundó, pouco acima da cachoeira; maximumElevationInMeters: 795; verbatimCoordinates: $3^{\circ} 50^{\prime} 13^{\prime \prime S}, 40^{\circ} 54^{\prime} 35 " \mathrm{~W}$; samplingProtocol: Malaise intercept trap; verbatimEventDate: 21.iv.12; individualCount: 1; sex: male; lifeStage: adult; recordedBy: Rafael, J.A. | Limeira-de-Oliveira, F. | Takiya, D.M. | et al.; identifiedBy: Allan Paulo Moreira dos Santos; institutionCode: DZRJ; basisOfRecord: PreservedSpecimen

Distribution: Brazil: CE.

Notes: Species described in Souza et al. 2014a.

\section{Ochrotrichia patulosa (Wasmund \& Holzenthal, 2007)}

\section{Material}

a. country: Brazil; stateProvince: Ceará; municipality: Ubajara; locality: Parque Nacional de Ubajara, Rio Cafundó, pouco acima da cachoeira; maximumElevationInMeters: 795; verbatimCoordinates: $3^{\circ} 50^{\prime} 13^{\prime \prime S}, 40^{\circ} 54^{\prime} 35 \mathrm{~W} \mathrm{~W}$; samplingProtocol: Malaise intercept trap; verbatimEventDate: 13.xi.12; individualCount: 1; sex: male; lifeStage: adult; recordedBy: Limeira-de-Oliveira | et al.; identifiedBy: Wagner Rafael Maciel de Souza; institutionCode: DZRJ; basisOfRecord: PreservedSpecimen

Distribution: Brazil: CE, RJ.

Notes: Species firstly recorded from CE in Souza et al. 2014a.

\section{Genus Oxyethira Eaton, 1873}

Notes: New genus record for CE.

\section{Oxyethira parce (Edwards \& Arnold, 1961)}

\section{Materials}

a. $\quad$ country: Brazil; stateProvince: Ceará; municipality: Ubajara; locality: Parque Nacional de Ubajara, Trilha Araticum, Rio das Minas; maximumElevationInMeters: 524; verbatimCoordinates: $3^{\circ} 50^{\prime} 3$ "S, $40^{\circ} 54^{\prime} 18^{\prime \prime} \mathrm{W}$; samplingProtocol: Malaise intercept trap; verbatimEventDate: 14.ii.13; individualCount: 2; sex: male; lifeStage: adult; recordedBy: Rafael, J.A. | Limeira-de-Oliveira, F. | Takiya, D.M. | Santos, A.P.M. | et al.; identifiedBy: Allan Paulo Moreira dos Santos; institutionCode: DZRJ; basisOfRecord: PreservedSpecimen

b. country: Brazil; stateProvince: Ceará; municipality: Ubajara; locality: Parque Nacional de Ubajara, Trilha Araticum, Rio das Minas; maximumElevationInMeters: 524; verbatimCoordinates: $3^{\circ} 50^{\prime} 3$ "S, $40^{\circ} 54^{\prime} 18^{\prime \prime} \mathrm{W}$; sampling Protocol: Malaise intercept trap; verbatimEventDate: 17.ii.13; individualCount: 1; sex: male; lifeStage: adult; recordedBy: Rafael, J.A. | Limeira-de-Oliveira, F. | Takiya, D.M. | Santos, A.P.M. | et al.; identifiedBy: Allan Paulo Moreira dos Santos; institutionCode: DZRJ; basisOfRecord: PreservedSpecimen 
c. country: Brazil; stateProvince: Ceará; municipality: Ubajara; locality: Parque Nacional de Ubajara, Trilha Araticum, Rio das Minas; maximumElevationInMeters: 524; verbatimCoordinates: $3^{\circ} 50^{\prime} 3 " \mathrm{~S}, 40^{\circ} 54^{\prime} 18^{\prime \prime} \mathrm{W}$; samplingProtocol: Pennsylvania light trap; verbatimEventDate: 22.iv.12; individualCount: 2; sex: male; lifeStage: adult; recordedBy: Takiya, D.M. | Somavilla, A.; identifiedBy: Allan Paulo Moreira dos Santos; institutionCode: DZRJ; basisOfRecord: PreservedSpecimen

Distribution: USA, Mexico. Costa Rica. Panama. Trinidad and Tobago. Colombia. Venezuela. Guyana. Brazil: CE!, MG. Ecuador. Peru. Bolivia. Argentina.

Notes: New species record for Northeastern Brazil.

\section{Oxyethira tica* Harris \& Holzenthal, 1992}

\section{Materials}

a. country: Brazil; stateProvince: Ceará; municipality: Ubajara; locality: Parque Nacional de Ubajara, Trilha Araticum, Rio das Minas; maximumElevationInMeters: 524; verbatimCoordinates: $3^{\circ} 50^{\prime} 3$ "S, $40^{\circ} 54^{\prime} 18 \mathrm{~W} \mathrm{~W}$; samplingProtocol: Malaise intercept trap; verbatimEventDate: 14.ii.13; individualCount: 1; sex: male; lifeStage: adult; recordedBy: Rafael, J.A. | Limeira-de-Oliveira, F. | Takiya, D.M. | Santos, A.P.M. | et al.; identifiedBy: Allan Paulo Moreira dos Santos; institutionCode: DZRJ; basisOfRecord: PreservedSpecimen

b. $\quad$ country: Brazil; stateProvince: Ceará; municipality: Ubajara; locality: Parque Nacional de Ubajara, Trilha Araticum, Rio das Minas na altura da trilha do teleférico; maximumElevationInMeters: 420; verbatimCoordinates: $3^{\circ} 49^{\prime} 58^{\prime \prime S}, 40^{\circ} 53^{\prime} 53 " \mathrm{~W}$; samplingProtocol: Malaise intercept trap; verbatimEventDate: 14.ii.13; individualCount: 1; sex: male; lifeStage: adult; recordedBy: Rafael, J.A. | Limeira-de-Oliveira, F. | Takiya, D.M. | Santos, A.P.M. | et al.; identifiedBy: Allan Paulo Moreira dos Santos; institutionCode: DZRJ; basisOfRecord: PreservedSpecimen

c. country: Brazil; stateProvince: Ceará; municipality: Ubajara; locality: Parque Nacional de Ubajara, Trilha Araticum, Rio das Minas na altura da trilha do teleférico; maximumElevationInMeters: 420; verbatimCoordinates: $3^{\circ} 49^{\prime} 58^{\prime \prime S}, 40^{\circ} 53^{\prime} 53^{\prime \prime} \mathrm{W}$; samplingProtocol: Malaise intercept trap; verbatimEventDate: 14.ii.13; individualCount: 1; sex: female; lifeStage: adult; recordedBy: Rafael, J.A. | Limeira-de-Oliveira, F. | Takiya, D.M. | Santos, A.P.M. | et al.; identifiedBy: Allan Paulo Moreira dos Santos; institutionCode: DZRJ; basisOfRecord: PreservedSpecimen

d. country: Brazil; stateProvince: Ceará; municipality: Ubajara; locality: Parque Nacional de Ubajara, Rio das Minas, próximo ao Portão Araticum; maximumElevationInMeters: 328; verbatimCoordinates: $3^{\circ} 49^{\prime} 32.6^{\prime \prime S}, 40^{\circ} 53^{\prime} 32.8^{\prime \prime W}$; samplingProtocol: Malaise intercept trap; verbatimEventDate: 17.ii.13; individualCount: 1; sex: male; lifeStage: adult; recordedBy: Rafael, J.A. | Limeira-de-Oliveira, F. | Takiya, D.M. | Santos, A.P.M. | et al.; identifiedBy: Allan Paulo Moreira dos Santos; institutionCode: DZRJ; basisOfRecord: PreservedSpecimen

e. $\quad$ country: Brazil; stateProvince: Ceará; municipality: Ubajara; locality: Parque Nacional de Ubajara, Trilha Araticum, Rio das Minas; maximumElevationInMeters: 524; verbatimCoordinates: $3^{\circ} 50^{\prime} 3$ "S, $40^{\circ} 54^{\prime} 18$ "W; samplingProtocol: Pennsylvania light trap; verbatimEventDate: 22.iv.12; individualCount: 1; sex: male; lifeStage: adult; recordedBy: Takiya, D.M. | Somavilla, A.; identifiedBy: Allan Paulo Moreira dos Santos; institutionCode: DZRJ; basisOfRecord: PreservedSpecimen 
f. country: Brazil; stateProvince: Ceará; municipality: Ubajara; locality: Parque Nacional de Ubajara, Rio Cafundó, pouco acima da cachoeira; maximumElevationInMeters: 795; verbatimCoordinates: $3^{\circ} 50^{\prime} 13^{\prime \prime} \mathrm{S}, 40^{\circ} 54^{\prime} 35 " \mathrm{~W}$; sampling Protocol: Pennsylvania light trap; verbatimEventDate: 24.iv.12; individualCount: 1; sex: male; lifeStage: adult; recordedBy: Takiya, D.M. | Rafael, J.A.; identifiedBy: Allan Paulo Moreira dos Santos; institutionCode: DZRJ; basisOfRecord: PreservedSpecimen

Distribution: Mexico. Honduras. Costa Rica. Panama. Guadeloupe. Dominica. Santa Lucia. Saint Vicent and the Grenadines. Granada. Trinidad and Tobago. Venezuela. Brazil: AM, PI!, CE!, MG, RJ. Ecuador.

Notes: New species record for Northeastern Brazil.

\section{Family Leptoceridae}

\section{Genus Atanatolica Mosely, 1936}

Notes: Genus firstly recorded from CE in Henriques-Oliveira and Santos 2014.

\section{Atanatolica nordestina Henriques-Oliveira \& Santos, 2014}

\section{Materials}

a. country: Brazil; stateProvince: Ceará; municipality: Ubajara; locality: Parque Nacional de Ubajara, Rio Cafundó, pouco acima da cachoeira; maximumElevationInMeters: 795; verbatimCoordinates: $3^{\circ} 50^{\prime} 13^{\prime \prime} \mathrm{S}, 40^{\circ} 54^{\prime} 35 \mathrm{WW}$; samplingProtocol: Malaise intercept trap; verbatimEventDate: 1.ii.13; individualCount: 2; sex: male; lifeStage: adult; recordedBy: Limeira-de-Oliveira | et al.; identifiedBy: Ana Lucia Henriques Oliveira | Allan Paulo Moreira dos Santos; institutionCode: DZRJ; basisOfRecord: PreservedSpecimen

b. $\quad$ country: Brazil; stateProvince: Ceará; municipality: Ubajara; locality: Parque Nacional de Ubajara, Rio Cafundó, pouco acima da cachoeira; maximumElevationInMeters: 795; verbatimCoordinates: $3^{\circ} 50^{\prime} 13^{\prime \prime S}, 40^{\circ} 54^{\prime} 35^{\prime \prime W}$; samplingProtocol: Malaise intercept trap; verbatimEventDate: 1.ii.13; individualCount: 1; sex: female; lifeStage: adult; recordedBy: Limeira-de-Oliveira | et al.; identifiedBy: Ana Lucia Henriques Oliveira | Allan Paulo Moreira dos Santos; institutionCode: DZRJ; basisOfRecord: PreservedSpecimen

c. country: Brazil; stateProvince: Ceará; municipality: Ubajara; locality: Parque Nacional de Ubajara, Rio Cafundó, pouco acima da cachoeira; maximumElevationInMeters: 795; verbatimCoordinates: $3^{\circ} 50^{\prime} 13^{\prime \prime} \mathrm{S}, 40^{\circ} 54^{\prime} 35^{\prime \prime} \mathrm{W}$; samplingProtocol: Malaise intercept trap; verbatimEventDate: 13.xi.12; individualCount: 2; sex: female; lifeStage: adult; recordedBy: Limeira-de-Oliveira | et al.; identifiedBy: Ana Lucia Henriques Oliveira | Allan Paulo Moreira dos Santos; institutionCode: DZRJ; basisOfRecord: PreservedSpecimen

d. country: Brazil; stateProvince: Ceará; municipality: Ubajara; locality: Parque Nacional de Ubajara, Cachoeira do Cafundó; maximumElevationInMeters: 783; verbatimCoordinates: 350'12"S, 4054'35"W; samplingProtocol: Manual; verbatimEventDate: 16.ii.13; individualCount: 1; sex: male; lifeStage: adult; recordedBy: Santos, A.P.M. | Takiya, D.M.; identifiedBy: Ana Lucia Henriques Oliveira | Allan Paulo Moreira dos Santos; institutionCode: DZRJ; basisOfRecord: PreservedSpecimen

e. country: Brazil; stateProvince: Ceará; municipality: Ubajara; locality: Parque Nacional de Ubajara, Trilha Araticum, Rio Cafundó; maximumElevationInMeters: 753; 
verbatimCoordinates: $3^{\circ} 50^{\prime} 12^{\prime \prime S}, 40^{\circ} 54^{\prime} 31$ "W; samplingProtocol: Manual; verbatimEventDate: 17.ii.13; individualCount: 1; sex: male; lifeStage: adult; recordedBy: Santos, A.P.M. | Takiya, D.M.; identifiedBy: Ana Lucia Henriques Oliveira| Allan Paulo Moreira dos Santos; institutionCode: DZRJ; basisOfRecord: PreservedSpecimen

f. country: Brazil; stateProvince: Ceará; municipality: Ubajara; locality: Parque Nacional de Ubajara, Trilha Araticum, Rio Cafundó; maximumElevationInMeters: 753; verbatimCoordinates: $3^{\circ} 50^{\prime} 12$ "S, 4054'31"W; samplingProtocol: Manual; verbatimEventDate: 17.ii.13; individualCount: 12; lifeStage: immature; recordedBy: Santos, A.P.M. | Takiya, D.M.; identifiedBy: Ana Lucia Henriques Oliveira | Allan Paulo Moreira dos Santos; institutionCode: DZRJ; basisOfRecord: PreservedSpecimen

g. country: Brazil; stateProvince: Ceará; municipality: Ubajara; locality: Parque Nacional de Ubajara, Rio das Minas, próximo ao Portão Araticum; maximumElevationInMeters: 328; verbatimCoordinates: $3^{\circ} 49^{\prime} 32.6^{\prime \prime S}, 40^{\circ} 53^{\prime} 32.8 \mathrm{WW}$; samplingProtocol: Manual; verbatimEventDate: 17.ii.13; individualCount: 11; lifeStage: immature; recordedBy: Santos, A.P.M. | Takiya, D.M.; identifiedBy: Ana Lucia Henriques Oliveira | Allan Paulo Moreira dos Santos; institutionCode: DZRJ; basisOfRecord: PreservedSpecimen

h. country: Brazil; stateProvince: Ceará; municipality: Ubajara; locality: Parque Nacional de Ubajara, Rio Cafundó, pouco acima da cachoeira; maximumElevationInMeters: 795; verbatimCoordinates: $3^{\circ} 50^{\prime} 13^{\prime \prime} \mathrm{S}, 40^{\circ} 54^{\prime} 35 " \mathrm{~W}$; samplingProtocol: Malaise intercept trap; verbatimEventDate: 18.xi.12; individualCount: 3; sex: male; lifeStage: adult; recordedBy: Limeira-de-Oliveira | et al.; identifiedBy: Ana Lucia Henriques Oliveira | Allan Paulo Moreira dos Santos; institutionCode: DZRJ; basisOfRecord: PreservedSpecimen

i. country: Brazil; stateProvince: Ceará; municipality: Ubajara; locality: Parque Nacional de Ubajara, Rio Cafundó, pouco acima da cachoeira; maximumElevationInMeters: 795; verbatimCoordinates: $3^{\circ} 50^{\prime} 13^{\prime \prime} \mathrm{S}, 40^{\circ} 54^{\prime} 35 " \mathrm{~W}$; sampling Protocol: Malaise intercept trap; verbatimEventDate: 18.xi.12; individualCount: 3; sex: female; lifeStage: adult; recordedBy: Limeira-de-Oliveira | et al.; identifiedBy: Ana Lucia Henriques Oliveira | Allan Paulo Moreira dos Santos; institutionCode: DZRJ; basisOfRecord: PreservedSpecimen

j. country: Brazil; stateProvince: Ceará; municipality: Ubajara; locality: Parque Nacional de Ubajara, Trilha Araticum, Rio das Minas na altura da trilha do teleférico; maximumElevationInMeters: 420; verbatimCoordinates: $3^{\circ} 49^{\prime} 58^{\prime \prime S}, 40^{\circ} 53^{\prime} 53^{\prime \prime} \mathrm{W}$; samplingProtocol: Malaise intercept trap; verbatimEventDate: 20.iv.12; individualCount: 2; sex: male; lifeStage: adult; recordedBy: Rafael, J.A. | Limeira-de-Oliveira, F. | Takiya, D.M. | et al.; identifiedBy: Ana Lucia Henriques Oliveira| Allan Paulo Moreira dos Santos; institutionCode: DZRJ; basisOfRecord: PreservedSpecimen

k. country: Brazil; stateProvince: Ceará; municipality: Ubajara; locality: Parque Nacional de Ubajara, Rio Cafundó, pouco acima da cachoeira; maximumElevationInMeters: 795; verbatimCoordinates: $3^{\circ} 50^{\prime} 13^{\prime \prime} \mathrm{S}, 40^{\circ} 54^{\prime} 35 \mathrm{~W} \mathrm{~W}$; samplingProtocol: Pennsylvania light trap; verbatimEventDate: 24.iv.12; individualCount: 1; sex: male; lifeStage: adult; recordedBy: Takiya, D.M. | Rafael, J.A.; identifiedBy: Ana Lucia Henriques Oliveira | Allan Paulo Moreira dos Santos; institutionCode: DZRJ; basisOfRecord: PreservedSpecimen

Distribution: Brazil: CE.

Notes: Species described in Henriques-Oliveira and Santos 2014. See Fig. 26. 


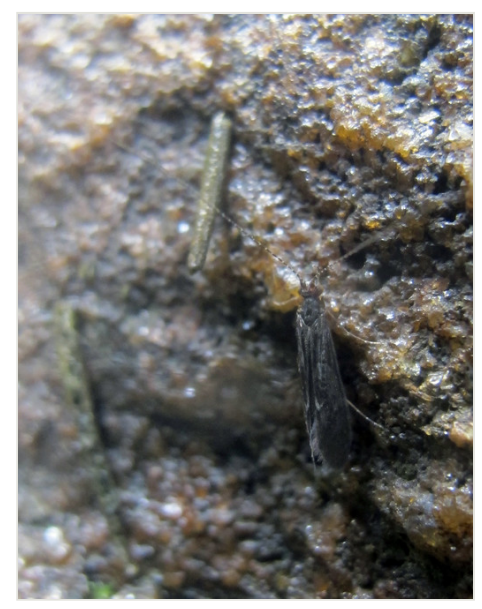

Figure 26.

Adult and immature specimens of Atanatolica nordestina collected at PNU-04, species described based on material collected during this project.

\section{Genus Nectopsyche Müller, 1879}

\section{Nectopsyche sp.}

\section{Material}

a. country: Brazil; stateProvince: Ceará; municipality: Ubajara; locality: Parque Nacional de Ubajara, Trilha Araticum, Rio Cafundó; maximumElevationInMeters: 753; verbatimCoordinates: $3^{\circ} 50^{\prime} 12$ "S, 4054'31"W; samplingProtocol: Manual; verbatimEventDate: 23.iv.12; individualCount: 10; lifeStage: immature; recordedBy: Takiya, D.M.; identifiedBy: Allan Paulo Moreira dos Santos; institutionCode: DZRJ; basisOfRecord: PreservedSpecimen

\section{Genus Oecetis McLachlan, 1877}

\section{Oecetis fibra Chen \& Morse, 2012}

\section{Materials}

a. country: Brazil; stateProvince: Ceará; municipality: Ubajara; locality: Parque Nacional de Ubajara, Trilha Samambaia, Rio Gameleira; maximumElevationInMeters: 874; verbatimCoordinates: $3^{\circ} 50^{\prime} 25^{\prime \prime}$ S, $40^{\circ} 54^{\prime} 19^{\prime \prime} \mathrm{W}$; samplingProtocol: Pennsylvania light trap; verbatimEventDate: 13.ii.13; individualCount: 1; sex: male; lifeStage: adult; recordedBy: Santos, A.P.M. | Takiya, D.M.; identifiedBy: Allan Paulo Moreira dos Santos; institutionCode: DZRJ; basisOfRecord: PreservedSpecimen

b. $\quad$ country: Brazil; stateProvince: Ceará; municipality: Ubajara; locality: Parque Nacional de Ubajara, Rio Cafundó, pouco acima da cachoeira; maximumElevationInMeters: 795; verbatimCoordinates: $3^{\circ} 50^{\prime} 13^{\prime \prime} \mathrm{S}, 40^{\circ} 54^{\prime} 35^{\prime \prime} \mathrm{W}$; samplingProtocol: Malaise intercept trap; 
verbatimEventDate: 13.xi.12; individualCount: 2; sex: male; lifeStage: adult; recordedBy: Limeira-de-Oliveira | et al.; identifiedBy: Ana Lucia Henriques Oliveira; institutionCode: DZRJ; basisOfRecord: PreservedSpecimen

c. country: Brazil; stateProvince: Ceará; municipality: Ubajara; locality: Parque Nacional de Ubajara, Rio Cafundó, pouco acima da cachoeira; maximumElevationInMeters: 795; verbatimCoordinates: $3^{\circ} 50^{\prime} 13^{\prime \prime S}, 40^{\circ} 54^{\prime} 35 \mathrm{WW}$; samplingProtocol: Malaise intercept trap; verbatimEventDate: 13.xi.12; individualCount: 1; sex: female; lifeStage: adult; recordedBy: Limeira-de-Oliveira | et al.; identifiedBy: Ana Lucia Henriques Oliveira; institutionCode: DZRJ; basisOfRecord: PreservedSpecimen

d. country: Brazil; stateProvince: Ceará; municipality: Ubajara; locality: Parque Nacional de Ubajara, Trilha Samambaia, Rio Gameleira; maximumElevationInMeters: 874; verbatimCoordinates: $3^{\circ} 50^{\prime 2} 25^{\prime} \mathrm{S}, 40^{\circ} 54^{\prime} 19$ "W; samplingProtocol: Pennsylvania light trap; verbatimEventDate: 14.ii.13; individualCount: 1; sex: female; lifeStage: adult; recordedBy: Santos, A.P.M. | Takiya, D.M.; identifiedBy: Ana Lucia Henriques Oliveira; institutionCode: DZRJ; basisOfRecord: PreservedSpecimen

e. country: Brazil; stateProvince: Ceará; municipality: Ubajara; locality: Parque Nacional de Ubajara, Rio Cafundó, pouco acima da cachoeira; maximumElevationInMeters: 795; verbatimCoordinates: $3^{\circ} 50^{\prime} 13^{\prime \prime S}, 40^{\circ} 54^{\prime} 35 " \mathrm{~W}$; samplingProtocol: Malaise intercept trap; verbatimEventDate: 18.xi.12; individualCount: 1; sex: male; lifeStage: adult; recordedBy: Limeira-de-Oliveira | et al.; identifiedBy: Ana Lucia Henriques Oliveira; institutionCode: DZRJ; basisOfRecord: PreservedSpecimen

f. country: Brazil; stateProvince: Ceará; municipality: Ubajara; locality: Parque Nacional de Ubajara, Rio Cafundó, pouco acima da cachoeira; maximumElevationInMeters: 795; verbatimCoordinates: $3^{\circ} 50^{\prime} 13^{\prime \prime} \mathrm{S}, 40^{\circ} 54^{\prime} 35 " \mathrm{~W}$; samplingProtocol: Malaise intercept trap; verbatimEventDate: 18.xi.12; individualCount: 1; sex: female; lifeStage: adult; recordedBy: Limeira-de-Oliveira | et al.; identifiedBy: Ana Lucia Henriques Oliveira; institutionCode: DZRJ; basisOfRecord: PreservedSpecimen

Distribution: Brazil: CE!, ES, SP, RJ, PR, SC.

Notes: New species record for Northeastern Brazil.

\section{Family Odontoceridae}

\section{Genus Marilia Müller, 1880}

\section{Marilia flexuosa Ulmer, 1905}

\section{Materials}

a. country: Brazil; stateProvince: Ceará; municipality: Ubajara; locality: Parque Nacional de Ubajara, Trilha Samambaia, Rio Gameleira; maximumElevationInMeters: 874; verbatimCoordinates: $3^{\circ} 50^{\prime 25}$ "S, 4054'19"W; samplingProtocol: Pennsylvania light trap; verbatimEventDate: 13.ii.13; individualCount: 2; sex: female; lifeStage: adult; recordedBy: Santos, A.P.M. | Takiya, D.M.; identifiedBy: Allan Paulo Moreira dos Santos; institutionCode: DZRJ; basisOfRecord: PreservedSpecimen

b. $\quad$ country: Brazil; stateProvince: Ceará; municipality: Ubajara; locality: Parque Nacional de Ubajara, Trilha Samambaia, Rio Gameleira; maximumElevationInMeters: 874; verbatimCoordinates: $3^{\circ} 50^{\prime} 25^{\prime \prime}$ S, $40^{\circ} 54^{\prime} 19^{\prime \prime}$; samplingProtocol: Pennsylvania light trap; 
verbatimEventDate: 14.ii.13; individualCount: 1; sex: male; lifeStage: adult; recordedBy: Santos, A.P.M. | Takiya, D.M.; identifiedBy: Allan Paulo Moreira dos Santos; institutionCode: DZRJ; basisOfRecord: PreservedSpecimen

c. country: Brazil; stateProvince: Ceará; municipality: Ubajara; locality: Parque Nacional de Ubajara, Trilha Samambaia, Rio Gameleira; maximumElevationInMeters: 874; verbatimCoordinates: $3^{\circ} 50^{\prime} 25^{\prime \prime}$ S, $40^{\circ} 54^{\prime} 19^{\prime \prime}$; samplingProtocol: Pennsylvania light trap; verbatimEventDate: 14.ii.13; individualCount: 1; sex: female; lifeStage: adult; recordedBy: Santos, A.P.M. | Takiya, D.M.; identifiedBy: Allan Paulo Moreira dos Santos; institutionCode: DZRJ; basisOfRecord: PreservedSpecimen

d. country: Brazil; stateProvince: Ceará; municipality: Ubajara; locality: Parque Nacional de Ubajara, Trilha Araticum, Rio das Minas; maximumElevationInMeters: 524; verbatimCoordinates: $3^{\circ} 50^{\prime} 3$ "S, $40^{\circ} 54^{\prime} 18 \mathrm{~W}$; samplingProtocol: Pennsylvania light trap; verbatimEventDate: 14.ii.13; individualCount: 1; sex: male; lifeStage: adult; recordedBy: Santos, A.P.M. | Takiya, D.M.; identifiedBy: Allan Paulo Moreira dos Santos; institutionCode: DZRJ; basisOfRecord: PreservedSpecimen

e. $\quad$ country: Brazil; stateProvince: Ceará; municipality: Ubajara; locality: Parque Nacional de Ubajara, Trilha Araticum, Rio das Minas; maximumElevationInMeters: 524; verbatimCoordinates: $3^{\circ} 50^{\prime} 3$ "S, $40^{\circ} 54^{\prime} 18^{\prime \prime W}$; samplingProtocol: Malaise intercept trap; verbatimEventDate: 14.ii.13; individualCount: 6; sex: male; lifeStage: adult; recordedBy: Rafael, J.A. | Limeira-de-Oliveira, F. | Takiya, D.M. | Santos, A.P.M. | et al.; identifiedBy: Allan Paulo Moreira dos Santos; institutionCode: DZRJ; basisOfRecord: PreservedSpecimen

f. country: Brazil; stateProvince: Ceará; municipality: Ubajara; locality: Parque Nacional de Ubajara, Trilha Araticum, Rio das Minas; maximumElevationInMeters: 524; verbatimCoordinates: $3^{\circ} 50^{\prime} 3$ "S, $40^{\circ} 54^{\prime} 18^{\prime \prime W}$; samplingProtocol: Malaise intercept trap; verbatimEventDate: 14.ii.13; individualCount: 6; sex: female; lifeStage: adult; recordedBy: Rafael, J.A. | Limeira-de-Oliveira, F. | Takiya, D.M. | Santos, A.P.M. | et al.; identifiedBy: Allan Paulo Moreira dos Santos; institutionCode: DZRJ; basisOfRecord: PreservedSpecimen

g. country: Brazil; stateProvince: Ceará; municipality: Ubajara; locality: Parque Nacional de Ubajara, Trilha Araticum, Rio das Minas na altura da trilha do teleférico; maximumElevationInMeters: 420 ; verbatimCoordinates: $3^{\circ} 49^{\prime} 58^{\prime \prime S}, 40^{\circ} 53^{\prime} 53^{\prime \prime} \mathrm{W}$; samplingProtocol: Malaise intercept trap; verbatimEventDate: 14.ii.13; individualCount: 4; sex: male; lifeStage: adult; recordedBy: Rafael, J.A. | Limeira-de-Oliveira, F. | Takiya, D.M. | Santos, A.P.M. | et al.; identifiedBy: Allan Paulo Moreira dos Santos; institutionCode: DZRJ; basisOfRecord: PreservedSpecimen

h. country: Brazil; stateProvince: Ceará; municipality: Ubajara; locality: Parque Nacional de Ubajara, Trilha Araticum, Rio das Minas na altura da trilha do teleférico; maximumElevationInMeters: 420; verbatimCoordinates: $3^{\circ} 49^{\prime} 58^{\prime \prime} \mathrm{S}, 40^{\circ} 53^{\prime} 53^{\prime \prime} \mathrm{W}$; samplingProtocol: Malaise intercept trap; verbatimEventDate: 14.ii.13; individualCount: 6; sex: female; lifeStage: adult; recordedBy: Rafael, J.A. | Limeira-de-Oliveira, F. | Takiya, D.M. | Santos, A.P.M. | et al.; identifiedBy: Allan Paulo Moreira dos Santos; institutionCode: DZRJ; basisOfRecord: PreservedSpecimen

i. country: Brazil; stateProvince: Ceará; municipality: Ubajara; locality: Parque Nacional de Ubajara, Trilha Araticum, Rio da Minas abaixo do teleférico; maximumElevationInMeters: 395; verbatimCoordinates: $3^{\circ} 49^{\prime} 43.3^{\prime \prime S}, 40^{\circ} 53^{\prime} 51.5^{\prime \prime W}$; samplingProtocol: Pennsylvania light trap; verbatimEventDate: 14.ii.13; individualCount: 3; sex: male; lifeStage: adult; recordedBy: Santos, A.P.M. | Takiya, D.M.; identifiedBy: Allan Paulo Moreira dos Santos; institutionCode: DZRJ; basisOfRecord: PreservedSpecimen 
j. country: Brazil; stateProvince: Ceará; municipality: Ubajara; locality: Parque Nacional de Ubajara, Trilha Araticum, Rio da Minas abaixo do teleférico; maximumElevationInMeters: 395; verbatimCoordinates: $3^{\circ} 49^{\prime} 43.3^{\prime \prime S}, 40^{\circ} 53^{\prime} 51.5 " \mathrm{~W}$; samplingProtocol: Pennsylvania light trap; verbatimEventDate: 14.ii.13; individualCount: 2; sex: female; lifeStage: adult; recordedBy: Santos, A.P.M. | Takiya, D.M.; identifiedBy: Allan Paulo Moreira dos Santos; institutionCode: DZRJ; basisOfRecord: PreservedSpecimen

k. country: Brazil; stateProvince: Ceará; municipality: Ubajara; locality: Parque Nacional de Ubajara, Rio das Minas, próximo ao Portão Araticum; maximumElevationInMeters: 328; verbatimCoordinates: $3^{\circ} 49^{\prime} 32.6^{\prime \prime S}, 40^{\circ} 53^{\prime} 32.8^{\prime \prime} \mathrm{W}$; samplingProtocol: Malaise intercept trap; verbatimEventDate: 14.ii.13; individualCount: 1; sex: male; lifeStage: adult; recordedBy: Rafael, J.A. | Limeira-de-Oliveira, F. | Takiya, D.M. | Santos, A.P.M. | et al.; identifiedBy: Allan Paulo Moreira dos Santos; institutionCode: DZRJ; basisOfRecord: PreservedSpecimen

I. country: Brazil; stateProvince: Ceará; municipality: Ubajara; locality: Parque Nacional de Ubajara, Rio das Minas, próximo ao Portão Araticum; maximumElevationInMeters: 328; verbatimCoordinates: $3^{\circ} 49^{\prime} 32.6^{\prime \prime}$ S, 4053'32.8"W; samplingProtocol: Malaise intercept trap; verbatimEventDate: 14.ii.13; individualCount: 2; sex: male; lifeStage: adult; recordedBy: Rafael, J.A. | Limeira-de-Oliveira, F. | Takiya, D.M. | Santos, A.P.M. | et al.; identifiedBy: Allan Paulo Moreira dos Santos; institutionCode: DZRJ; basisOfRecord: PreservedSpecimen

m. country: Brazil; stateProvince: Ceará; municipality: Ubajara; locality: Parque Nacional de Ubajara, Rio das Minas, próximo ao Portão Araticum; maximumElevationInMeters: 328; verbatimCoordinates: $3^{\circ} 49^{\prime} 32.6^{\prime \prime} \mathrm{S}, 40^{\circ} 53^{\prime} 32.8^{\prime \prime} \mathrm{W}$; samplingProtocol: Malaise intercept trap; verbatimEventDate: 14.ii.13; individualCount: 2; sex: female; lifeStage: adult; recordedBy: Rafael, J.A. | Limeira-de-Oliveira, F. | Takiya, D.M. | Santos, A.P.M. | et al.; identifiedBy: Allan Paulo Moreira dos Santos; institutionCode: DZRJ; basisOfRecord: PreservedSpecimen

n. country: Brazil; stateProvince: Ceará; municipality: Ubajara; locality: Parque Nacional de Ubajara, Cachoeira do Cafundó; maximumElevationInMeters: 783; verbatimCoordinates: $3^{\circ} 50^{\prime} 12^{\prime \prime}$ S, $40^{\circ} 54^{\prime} 35 " \mathrm{~W}$; samplingProtocol: Pennsylvania light trap; verbatimEventDate: 15.ii.13; individualCount: 1; sex: female; lifeStage: adult; recordedBy: Santos, A.P.M. | Takiya, D.M.; identifiedBy: Allan Paulo Moreira dos Santos; institutionCode: DZRJ; basisOfRecord: PreservedSpecimen

o. country: Brazil; stateProvince: Ceará; municipality: Ubajara; locality: Parque Nacional de Ubajara, Trilha Samambaia, Rio Gameleira; maximumElevationInMeters: 874; verbatimCoordinates: $3^{\circ} 50$ '25"S, 4054'19"W; samplingProtocol: Malaise intercept trap; verbatimEventDate: 17.ii.13; individualCount: 1; sex: female; lifeStage: adult; recordedBy: Rafael, J.A. | Limeira-de-Oliveira, F. | Takiya, D.M. | Santos, A.P.M. | et al.; identifiedBy: Allan Paulo Moreira dos Santos; institutionCode: DZRJ; basisOfRecord: PreservedSpecimen

p. $\quad$ country: Brazil; stateProvince: Ceará; municipality: Ubajara; locality: Parque Nacional de Ubajara, Trilha Araticum, Rio das Minas; maximumElevationInMeters: 524; verbatimCoordinates: $3^{\circ} 50^{\prime} 3$ "S, $40^{\circ} 54^{\prime} 18 \mathrm{WW}$; samplingProtocol: Malaise intercept trap; verbatimEventDate: 17.ii.13; individualCount: 1; sex: female; lifeStage: adult; recordedBy: Rafael, J.A. | Limeira-de-Oliveira, F. | Takiya, D.M. | Santos, A.P.M. | et al.; identifiedBy: Allan Paulo Moreira dos Santos; institutionCode: DZRJ; basisOfRecord: PreservedSpecimen

q. country: Brazil; stateProvince: Ceará; municipality: Ubajara; locality: Parque Nacional de Ubajara, Rio das Minas, próximo ao Portão Araticum; maximumElevationInMeters: 328; verbatimCoordinates: $3^{\circ} 49^{\prime} 32.6^{\prime \prime} \mathrm{S}, 40^{\circ} 53^{\prime} 32.8^{\prime \prime W}$; samplingProtocol: Malaise intercept 
trap; verbatimEventDate: 17.ii.13; individualCount: 3; sex: male; lifeStage: adult; recordedBy: Rafael, J.A. | Limeira-de-Oliveira, F. | Takiya, D.M. | Santos, A.P.M. | et al.; identifiedBy: Allan Paulo Moreira dos Santos; institutionCode: DZRJ; basisOfRecord: PreservedSpecimen

r. country: Brazil; stateProvince: Ceará; municipality: Ubajara; locality: Parque Nacional de Ubajara, Rio das Minas, próximo ao Portão Araticum; maximumElevationInMeters: 328; verbatimCoordinates: $3^{\circ} 49^{\prime} 32.6^{\prime \prime S}, 40^{\circ} 53^{\prime} 32.8^{\prime \prime W}$; samplingProtocol: Malaise intercept trap; verbatimEventDate: 17.ii.13; individualCount: 2; sex: female; lifeStage: adult; recordedBy: Rafael, J.A. | Limeira-de-Oliveira, F. | Takiya, D.M. | Santos, A.P.M. | et al.; identifiedBy: Allan Paulo Moreira dos Santos; institutionCode: DZRJ; basisOfRecord: PreservedSpecimen

s. $\quad$ country: Brazil; stateProvince: Ceará; municipality: Ubajara; locality: Parque Nacional de Ubajara, Rio Cafundó, pouco acima da cachoeira; maximumElevationInMeters: 795; verbatimCoordinates: $3^{\circ} 50^{\prime} 13^{\prime \prime S}, 40^{\circ} 54^{\prime} 35 \mathrm{WW}$; samplingProtocol: Malaise intercept trap; verbatimEventDate: 19.i.13; individualCount: 1; sex: male; lifeStage: adult; recordedBy: Limeira-de-Oliveira | et al.; identifiedBy: Allan Paulo Moreira dos Santos; institutionCode: DZRJ; basisOfRecord: PreservedSpecimen

t. country: Brazil; stateProvince: Ceará; municipality: Ubajara; locality: Parque Nacional de Ubajara, Trilha Araticum, Rio das Minas na altura da trilha do teleférico; maximumElevationInMeters: 420; verbatimCoordinates: 349'58"S, 4053'53"W; samplingProtocol: Malaise intercept trap; verbatimEventDate: 20.iv.12; individualCount: 1; sex: male; lifeStage: adult; recordedBy: Rafael, J.A. | Limeira-de-Oliveira, F. | Takiya, D.M. | et al.; identifiedBy: Allan Paulo Moreira dos Santos; institutionCode: DZRJ; basisOfRecord: PreservedSpecimen

u. country: Brazil; stateProvince: Ceará; municipality: Ubajara; locality: Parque Nacional de Ubajara, Trilha Araticum, Rio das Minas na altura da trilha do teleférico; maximumElevationInMeters: 420; verbatimCoordinates: $3^{\circ} 49^{\prime} 58^{\prime \prime S}, 40^{\circ} 53^{\prime} 53^{\prime \prime} \mathrm{W}$; samplingProtocol: Malaise intercept trap; verbatimEventDate: 20.iv.12; individualCount: 1; sex: female; lifeStage: adult; recordedBy: Rafael, J.A. | Limeira-de-Oliveira, F. | Takiya, D.M. | et al.; identifiedBy: Allan Paulo Moreira dos Santos; institutionCode: DZRJ; basisOfRecord: PreservedSpecimen

v. country: Brazil; stateProvince: Ceará; municipality: Ubajara; locality: Parque Nacional de Ubajara, Trilha Araticum, Rio das Minas na altura da trilha do teleférico; maximumElevationInMeters: 420; verbatimCoordinates: $3^{\circ} 49^{\prime} 58^{\prime \prime S}, 40^{\circ} 53^{\prime} 53^{\prime \prime} \mathrm{W}$; samplingProtocol: Malaise intercept trap; verbatimEventDate: 20.iv.12; individualCount: 1; sex: male; lifeStage: adult; recordedBy: Rafael, J.A. | Limeira-de-Oliveira, F. | Takiya, D.M. | et al.; identifiedBy: Allan Paulo Moreira dos Santos; institutionCode: DZRJ; basisOfRecord: PreservedSpecimen

w. country: Brazil; stateProvince: Ceará; municipality: Ubajara; locality: Parque Nacional de Ubajara, Trilha Araticum, Rio das Minas na altura da trilha do teleférico; maximumElevationInMeters: 420; verbatimCoordinates: $3^{\circ} 49^{\prime} 58^{\prime \prime S}, 40^{\circ} 53^{\prime} 53 " \mathrm{~W}$; samplingProtocol: Malaise intercept trap; verbatimEventDate: 20.iv.12; individualCount: 2; sex: male; lifeStage: adult; recordedBy: Rafael, J.A. | Limeira-de-Oliveira, F. | Takiya, D.M. I et al.; identifiedBy: Allan Paulo Moreira dos Santos; institutionCode: DZRJ; basisOfRecord: PreservedSpecimen

x. country: Brazil; stateProvince: Ceará; municipality: Ubajara; locality: Parque Nacional de Ubajara, Trilha Araticum, Rio das Minas na altura da trilha do teleférico; maximumElevationInMeters: 420; verbatimCoordinates: $3^{\circ} 49^{\prime} 58^{\prime \prime S}, 40^{\circ} 53^{\prime} 53^{\prime \prime} \mathrm{W}$; samplingProtocol: Malaise intercept trap; verbatimEventDate: 20.iv.12; individualCount: 5; sex: female; lifeStage: adult; recordedBy: Rafael, J.A. | Limeira-de-Oliveira, F. | Takiya, 
D.M. | et al.; identifiedBy: Allan Paulo Moreira dos Santos; institutionCode: DZRJ; basisOfRecord: PreservedSpecimen

y. $\quad$ country: Brazil; stateProvince: Ceará; municipality: Ubajara; locality: Parque Nacional de Ubajara, Trilha Araticum, Rio das Minas na altura da trilha do teleférico; maximumElevationInMeters: 420; verbatimCoordinates: $3^{\circ} 49^{\prime} 58^{\prime \prime S}, 40^{\circ} 53^{\prime} 53^{\prime \prime} \mathrm{W}$; samplingProtocol: Malaise intercept trap; verbatimEventDate: 20.iv.12; individualCount: 1; sex: female; lifeStage: adult; recordedBy: Rafael, J.A. | Limeira-de-Oliveira, F. | Takiya, D.M. | et al.; identifiedBy: Allan Paulo Moreira dos Santos; institutionCode: DZRJ; basisOfRecord: PreservedSpecimen

z. country: Brazil; stateProvince: Ceará; municipality: Ubajara; locality: Parque Nacional de Ubajara, Trilha Araticum, Rio das Minas na altura da trilha do teleférico; maximumElevationInMeters: 420; verbatimCoordinates: $3^{\circ} 49^{\prime} 58^{\prime \prime S}, 40^{\circ} 53^{\prime} 53 " \mathrm{~W}$; samplingProtocol: Malaise intercept trap; verbatimEventDate: 21.v.12; individualCount: 1; sex: male; lifeStage: adult; recordedBy: Limeira-de-Oliveira | et al.; identifiedBy: Allan Paulo Moreira dos Santos; institutionCode: DZRJ; basisOfRecord: PreservedSpecimen

aa. country: Brazil; stateProvince: Ceará; municipality: Ubajara; locality: Parque Nacional de Ubajara, Trilha Araticum, Rio das Minas na altura da trilha do teleférico; maximumElevationInMeters: 420; verbatimCoordinates: $3^{\circ} 49^{\prime} 58^{\prime \prime} \mathrm{S}, 40^{\circ} 53^{\prime} 53^{\prime \prime} \mathrm{W}$; samplingProtocol: Malaise intercept trap; verbatimEventDate: 21.v.12; individualCount: 4; sex: female; lifeStage: adult; recordedBy: Limeira-de-Oliveira | et al.; identifiedBy: Allan Paulo Moreira dos Santos; institutionCode: DZRJ; basisOfRecord: PreservedSpecimen ab. country: Brazil; stateProvince: Ceará; municipality: Ubajara; locality: Parque Nacional de Ubajara, Trilha Samambaia, Mirante da cachoeira do Gameleira; maximumElevationInMeters: 880; verbatimCoordinates: $3^{\circ} 50^{\prime} 21$ 'S, 4054'23"W; samplingProtocol: Pennsylvania light trap; verbatimEventDate: 23.iv.12; individualCount: 5; sex: male; lifeStage: adult; recordedBy: Takiya, D.M. | Câmara, J.T.; identifiedBy: Allan Paulo Moreira dos Santos; institutionCode: DZRJ; basisOfRecord: PreservedSpecimen

Distribution: Canada. USA. Mexico. Guatemala. Nicaragua. Costa Rica. Panama. Colombia. Brazil: CE!. Peru. Argentina.

Notes: New species record for Northeastern Brazil.

\section{Genus Chimarra Stephens, 1829}

\section{Chimarra (Chimarra) calori Blahnik \& Holzenthal, 2012}

\section{Materials}

a. country: Brazil; stateProvince: Ceará; municipality: Ubajara; locality: Parque Nacional de Ubajara, Trilha Araticum, Rio das Minas; maximumElevationInMeters: 524; verbatimCoordinates: $3^{\circ} 50^{\prime} 3$ "S, $40^{\circ} 54^{\prime} 18^{\prime \prime} \mathrm{W}$; samplingProtocol: Malaise intercept trap; verbatimEventDate: 14.ii.13; individualCount: 4; sex: male; lifeStage: adult; recordedBy: Rafael, J.A. | Limeira-de-Oliveira, F. | Takiya, D.M. | Santos, A.P.M. | et al.; identifiedBy: Allan Paulo Moreira dos Santos; institutionCode: DZRJ; basisOfRecord: PreservedSpecimen

b. country: Brazil; stateProvince: Ceará; municipality: Ubajara; locality: Parque Nacional de Ubajara, Trilha Araticum, Rio das Minas; maximumElevationInMeters: 524; verbatimCoordinates: $3^{\circ} 50^{\prime} 3$ "S, $40^{\circ} 54^{\prime} 18^{\prime \prime W}$; sampling Protocol: Malaise intercept trap; verbatimEventDate: 14.ii.13; individualCount: 4; sex: female; lifeStage: adult; recordedBy: 
Rafael, J.A. | Limeira-de-Oliveira, F. | Takiya, D.M. | Santos, A.P.M. | et al.; identifiedBy: Allan Paulo Moreira dos Santos; institutionCode: DZRJ; basisOfRecord:

\section{PreservedSpecimen}

c. country: Brazil; stateProvince: Ceará; municipality: Ubajara; locality: Parque Nacional de Ubajara, Trilha Araticum, Rio das Minas na altura da trilha do teleférico; maximumElevationInMeters: 420; verbatimCoordinates: $3^{\circ} 49^{\prime} 58^{\prime \prime} \mathrm{S}, 40^{\circ} 53^{\prime} 53^{\prime \prime} \mathrm{W}$; samplingProtocol: Malaise intercept trap; verbatimEventDate: 14.ii.13; individualCount: 2; sex: male; lifeStage: adult; recordedBy: Rafael, J.A. | Limeira-de-Oliveira, F. | Takiya, D.M. | Santos, A.P.M. | et al.; identifiedBy: Allan Paulo Moreira dos Santos; institutionCode: DZRJ; basisOfRecord: PreservedSpecimen

d. country: Brazil; stateProvince: Ceará; municipality: Ubajara; locality: Parque Nacional de Ubajara, Trilha Araticum, Rio das Minas na altura da trilha do teleférico; maximumElevationInMeters: 420; verbatimCoordinates: $3^{\circ} 49^{\prime} 58^{\prime \prime} \mathrm{S}, 40^{\circ} 53^{\prime} 53^{\prime \prime} \mathrm{W}$; samplingProtocol: Malaise intercept trap; verbatimEventDate: 14.ii.13; individualCount: 6; sex: male; lifeStage: adult; recordedBy: Rafael, J.A. | Limeira-de-Oliveira, F. | Takiya, D.M. | Santos, A.P.M. | et al.; identifiedBy: Allan Paulo Moreira dos Santos; institutionCode: DZRJ; basisOfRecord: PreservedSpecimen

e. $\quad$ country: Brazil; stateProvince: Ceará; municipality: Ubajara; locality: Parque Nacional de Ubajara, Trilha Araticum, Rio das Minas na altura da trilha do teleférico; maximumElevationInMeters: 420 ; verbatimCoordinates: $3^{\circ} 49^{\prime} 58^{\prime \prime} \mathrm{S}, 40^{\circ} 53^{\prime} 53^{\prime \prime} \mathrm{W}$; samplingProtocol: Malaise intercept trap; verbatimEventDate: 14.ii.13; individualCount: 8; sex: female; lifeStage: adult; recordedBy: Rafael, J.A. | Limeira-de-Oliveira, F. | Takiya, D.M. | Santos, A.P.M. | et al.; identifiedBy: Allan Paulo Moreira dos Santos; institutionCode: DZRJ; basisOfRecord: PreservedSpecimen

f. country: Brazil; stateProvince: Ceará; municipality: Ubajara; locality: Parque Nacional de Ubajara, Rio das Minas, próximo ao Portão Araticum; maximumElevationInMeters: 328; verbatimCoordinates: $3^{\circ} 49^{\prime} 32.6^{\prime \prime S}, 40^{\circ} 53^{\prime} 32.8^{\prime \prime W}$; samplingProtocol: Malaise intercept trap; verbatimEventDate: 14.ii.13; individualCount: 4; sex: male; lifeStage: adult; recordedBy: Rafael, J.A. | Limeira-de-Oliveira, F. | Takiya, D.M. | Santos, A.P.M. | et al.; identifiedBy: Allan Paulo Moreira dos Santos; institutionCode: DZRJ; basisOfRecord: PreservedSpecimen

g. country: Brazil; stateProvince: Ceará; municipality: Ubajara; locality: Parque Nacional de Ubajara, Rio das Minas, próximo ao Portão Araticum; maximumElevationInMeters: 328; verbatimCoordinates: $3^{\circ} 49^{\prime} 32.6$ "S, 4053'32.8"W; samplingProtocol: Malaise intercept trap; verbatimEventDate: 14.ii.13; individualCount: 13; sex: female; lifeStage: adult; recordedBy: Rafael, J.A. | Limeira-de-Oliveira, F. | Takiya, D.M. | Santos, A.P.M. | et al.; identifiedBy: Allan Paulo Moreira dos Santos; institutionCode: DZRJ; basisOfRecord: PreservedSpecimen

h. country: Brazil; stateProvince: Ceará; municipality: Ubajara; locality: Parque Nacional de Ubajara, Trilha Araticum, Rio das Minas; maximumElevationInMeters: 524; verbatimCoordinates: $3^{\circ} 50^{\prime} 3$ "S, $40^{\circ} 54^{\prime} 18 " \mathrm{~W}$; sampling Protocol: Malaise intercept trap; verbatimEventDate: 17.ii.13; individualCount: 3; sex: male; lifeStage: adult; recordedBy: Rafael, J.A. | Limeira-de-Oliveira, F. | Takiya, D.M. | Santos, A.P.M. | et al.; identifiedBy: Allan Paulo Moreira dos Santos; institutionCode: DZRJ; basisOfRecord: PreservedSpecimen

i. country: Brazil; stateProvince: Ceará; municipality: Ubajara; locality: Parque Nacional de Ubajara, Rio das Minas, próximo ao Portão Araticum; maximumElevationlnMeters: 328; verbatimCoordinates: $3^{\circ} 49^{\prime} 32.6^{\prime \prime S}, 40^{\circ} 53^{\prime} 32.8^{\prime \prime} \mathrm{W}$; samplingProtocol: Malaise intercept trap; verbatimEventDate: 17.ii.13; individualCount: 10; sex: male; lifeStage: adult; recordedBy: Rafael, J.A. | Limeira-de-Oliveira, F. | Takiya, D.M. | Santos, A.P.M. | et al.; 
identifiedBy: Allan Paulo Moreira dos Santos; institutionCode: DZRJ; basisOfRecord: PreservedSpecimen

j. country: Brazil; stateProvince: Ceará; municipality: Ubajara; locality: Parque Nacional de Ubajara, Rio das Minas, próximo ao Portão Araticum; maximumElevationInMeters: 328; verbatimCoordinates: $3^{\circ} 49^{\prime} 32.6^{\prime \prime S}, 40^{\circ} 53^{\prime} 32.8^{\prime \prime W}$; samplingProtocol: Malaise intercept trap; verbatimEventDate: 17.ii.13; individualCount: 14; sex: female; lifeStage: adult; recordedBy: Rafael, J.A. | Limeira-de-Oliveira, F. | Takiya, D.M. | Santos, A.P.M. | et al.; identifiedBy: Allan Paulo Moreira dos Santos; institutionCode: DZRJ; basisOfRecord: PreservedSpecimen

k. $\quad$ country: Brazil; stateProvince: Ceará; municipality: Ubajara; locality: Parque Nacional de Ubajara, Trilha Araticum, Rio das Minas na altura da trilha do teleférico; maximumElevationInMeters: 420; verbatimCoordinates: $3^{\circ} 49^{\prime} 58^{\prime \prime S}, 40^{\circ} 53^{\prime} 53^{\prime \prime} \mathrm{W}$; samplingProtocol: Malaise intercept trap; verbatimEventDate: 20.iv.12; individualCount: 1; sex: male; lifeStage: adult; recordedBy: Rafael, J.A. | Limeira-de-Oliveira, F. | Takiya, D.M. | et al.; identifiedBy: Allan Paulo Moreira dos Santos; institutionCode: DZRJ; basisOfRecord: PreservedSpecimen

I. country: Brazil; stateProvince: Ceará; municipality: Ubajara; locality: Parque Nacional de Ubajara, Trilha Araticum, Rio das Minas na altura da trilha do teleférico; maximumElevationInMeters: 420; verbatimCoordinates: 349'58"S, 4053'53"W;

samplingProtocol: Malaise intercept trap; verbatimEventDate: 20.iv.12; individualCount: 5; sex: male; lifeStage: adult; recordedBy: Rafael, J.A. | Limeira-de-Oliveira, F. | Takiya, D.M. | et al.; identifiedBy: Allan Paulo Moreira dos Santos; institutionCode: DZRJ; basisOfRecord: PreservedSpecimen

Distribution: Brazil: CE!, MG, SP.

Notes: New species record for Northeastern Brazil.

\section{Chimarra (Curgia) conica Flint, 1983}

\section{Materials}

a. country: Brazil; stateProvince: Ceará; municipality: Ubajara; locality: Parque Nacional de Ubajara, Trilha Araticum, Rio das Minas na altura da trilha do teleférico; maximumElevationInMeters: 420; verbatimCoordinates: $3^{\circ} 49^{\prime} 58$ "S, 4053'53"W; samplingProtocol: Malaise intercept trap; verbatimEventDate: 14.ii.13; individualCount: 1; sex: male; lifeStage: adult; recordedBy: Rafael, J.A. | Limeira-de-Oliveira, F. | Takiya, D.M. I Santos, A.P.M. | et al.; identifiedBy: Allan Paulo Moreira dos Santos; institutionCode: DZRJ; basisOfRecord: PreservedSpecimen

b. country: Brazil; stateProvince: Ceará; municipality: Ubajara; locality: Parque Nacional de Ubajara, Trilha Araticum, Rio das Minas na altura da trilha do teleférico; maximumElevationInMeters: 420; verbatimCoordinates: $3^{\circ} 49^{\prime} 58^{\prime \prime S}, 40^{\circ} 53^{\prime} 53 " \mathrm{~W}$; samplingProtocol: Malaise intercept trap; verbatimEventDate: 14.ii.13; individualCount: 3; sex: male; lifeStage: adult; recordedBy: Rafael, J.A. | Limeira-de-Oliveira, F. | Takiya, D.M. | Santos, A.P.M. | et al.; identifiedBy: Allan Paulo Moreira dos Santos; institutionCode: DZRJ; basisOfRecord: PreservedSpecimen

c. country: Brazil; stateProvince: Ceará; municipality: Ubajara; locality: Parque Nacional de Ubajara, Trilha Araticum, Rio das Minas na altura da trilha do teleférico; maximumElevationInMeters: 420; verbatimCoordinates: $3^{\circ} 49^{\prime} 58$ "S, 4053'53"W; samplingProtocol: Malaise intercept trap; verbatimEventDate: 14.ii.13; individualCount: 5; sex: female; lifeStage: adult; recordedBy: Rafael, J.A. | Limeira-de-Oliveira, F. | Takiya, 
D.M. | Santos, A.P.M. | et al.; identifiedBy: Allan Paulo Moreira dos Santos; institutionCode: DZRJ; basisOfRecord: PreservedSpecimen

d. country: Brazil; stateProvince: Ceará; municipality: Ubajara; locality: Parque Nacional de Ubajara, Trilha Araticum, Rio das Minas na altura da trilha do teleférico; maximumElevationInMeters: 420; verbatimCoordinates: $3^{\circ} 49^{\prime} 58^{\prime \prime S}$, 4053'53"W; samplingProtocol: Malaise intercept trap; verbatimEventDate: 14.ii.13; individualCount: 25; sex: male; lifeStage: adult; recordedBy: Rafael, J.A. | Limeira-de-Oliveira, F. | Takiya, D.M. | Santos, A.P.M. | et al.; identifiedBy: Allan Paulo Moreira dos Santos; institutionCode: DZRJ; basisOfRecord: PreservedSpecimen

e. $\quad$ country: Brazil; stateProvince: Ceará; municipality: Ubajara; locality: Parque Nacional de Ubajara, Trilha Araticum, Rio das Minas na altura da trilha do teleférico; maximumElevationInMeters: 420; verbatimCoordinates: $3^{\circ} 49^{\prime} 58^{\prime \prime} \mathrm{S}, 40^{\circ} 53^{\prime} 53^{\prime \prime} \mathrm{W}$; samplingProtocol: Malaise intercept trap; verbatimEventDate: 14.ii.13; individualCount: 12; sex: female; lifeStage: adult; recordedBy: Rafael, J.A. | Limeira-de-Oliveira, F. | Takiya, D.M. | Santos, A.P.M. | et al.; identifiedBy: Allan Paulo Moreira dos Santos; institutionCode: DZRJ; basisOfRecord: PreservedSpecimen

f. country: Brazil; stateProvince: Ceará; municipality: Ubajara; locality: Parque Nacional de Ubajara, Rio das Minas, próximo ao Portão Araticum; maximumElevationInMeters: 328; verbatimCoordinates: $3^{\circ} 49^{\prime} 32.6^{\prime \prime S}, 40^{\circ} 53^{\prime} 32.8^{\prime \prime} \mathrm{W}$; samplingProtocol: Malaise intercept trap; verbatimEventDate: 14.ii.13; individualCount: 12; sex: male; lifeStage: adult; recordedBy: Rafael, J.A. | Limeira-de-Oliveira, F. | Takiya, D.M. | Santos, A.P.M. | et al.; identifiedBy: Allan Paulo Moreira dos Santos; institutionCode: DZRJ; basisOfRecord: PreservedSpecimen

g. country: Brazil; stateProvince: Ceará; municipality: Ubajara; locality: Parque Nacional de Ubajara, Rio das Minas, próximo ao Portão Araticum; maximumElevationInMeters: 328; verbatimCoordinates: $3^{\circ} 49^{\prime} 32.6^{\prime \prime S}, 40^{\circ} 53^{\prime} 32.8^{\prime \prime W}$; samplingProtocol: Malaise intercept trap; verbatimEventDate: 14.ii.13; individualCount: 39; sex: female; lifeStage: adult; recordedBy: Rafael, J.A. | Limeira-de-Oliveira, F. | Takiya, D.M. | Santos, A.P.M. | et al.; identifiedBy: Allan Paulo Moreira dos Santos; institutionCode: DZRJ; basisOfRecord: PreservedSpecimen

h. country: Brazil; stateProvince: Ceará; municipality: Ubajara; locality: Parque Nacional de Ubajara, Rio das Minas, próximo ao Portão Araticum; maximumElevationInMeters: 328; verbatimCoordinates: $3^{\circ} 49^{\prime} 32.6^{\prime \prime S}, 40^{\circ} 53^{\prime} 32.8^{\prime \prime}$; samplingProtocol: Malaise intercept trap; verbatimEventDate: 14.ii.13; individualCount: 19; sex: male; lifeStage: adult; recordedBy: Rafael, J.A. | Limeira-de-Oliveira, F. | Takiya, D.M. | Santos, A.P.M. | et al.; identifiedBy: Allan Paulo Moreira dos Santos; institutionCode: DZRJ; basisOfRecord: PreservedSpecimen

i. country: Brazil; stateProvince: Ceará; municipality: Ubajara; locality: Parque Nacional de Ubajara, Rio das Minas, próximo ao Portão Araticum; maximumElevationInMeters: 328; verbatimCoordinates: $3^{\circ} 49^{\prime} 32.6^{\prime \prime S}, 40^{\circ} 53^{\prime} 32.8^{\prime \prime W}$; samplingProtocol: Malaise intercept trap; verbatimEventDate: 14.ii.13; individualCount: 37; sex: female; lifeStage: adult; recordedBy: Rafael, J.A. | Limeira-de-Oliveira, F. | Takiya, D.M. | Santos, A.P.M. | et al.; identifiedBy: Allan Paulo Moreira dos Santos; institutionCode: DZRJ; basisOfRecord: PreservedSpecimen

j. country: Brazil; stateProvince: Ceará; municipality: Ubajara; locality: Parque Nacional de Ubajara, Trilha Araticum, Rio das Minas; maximumElevationInMeters: 524; verbatimCoordinates: $3^{\circ} 50^{\prime} 3$ "S, $40^{\circ} 54^{\prime} 18$ "W; samplingProtocol: Malaise intercept trap; verbatimEventDate: 17.ii.13; individualCount: 2; sex: male; lifeStage: adult; recordedBy: Rafael, J.A. | Limeira-de-Oliveira, F. | Takiya, D.M. | Santos, A.P.M. | et al.; identifiedBy: 
Allan Paulo Moreira dos Santos; institutionCode: DZRJ; basisOfRecord: PreservedSpecimen

k. country: Brazil; stateProvince: Ceará; municipality: Ubajara; locality: Parque Nacional de Ubajara, Rio das Minas, próximo ao Portão Araticum; maximumElevationInMeters: 328; verbatimCoordinates: $3^{\circ} 49^{\prime} 32.6^{\prime \prime S}, 40^{\circ} 53^{\prime} 32.8^{\prime \prime W}$; samplingProtocol: Malaise intercept trap; verbatimEventDate: 17.ii.13; individualCount: 19; sex: male; lifeStage: adult; recordedBy: Rafael, J.A. | Limeira-de-Oliveira, F. | Takiya, D.M. | Santos, A.P.M. | et al.; identifiedBy: Allan Paulo Moreira dos Santos; institutionCode: DZRJ; basisOfRecord: PreservedSpecimen

I. country: Brazil; stateProvince: Ceará; municipality: Ubajara; locality: Parque Nacional de Ubajara, Rio das Minas, próximo ao Portão Araticum; maximumElevationlnMeters: 328; verbatimCoordinates: $3^{\circ} 49^{\prime} 32.6$ "S, $40^{\circ} 53^{\prime} 32.8^{\prime \prime} \mathrm{W}$; samplingProtocol: Malaise intercept trap; verbatimEventDate: 17.ii.13; individualCount: 13; sex: female; lifeStage: adult; recordedBy: Rafael, J.A. | Limeira-de-Oliveira, F. | Takiya, D.M. | Santos, A.P.M. | et al.; identifiedBy: Allan Paulo Moreira dos Santos; institutionCode: DZRJ; basisOfRecord: PreservedSpecimen

m. country: Brazil; stateProvince: Ceará; municipality: Ubajara; locality: Parque Nacional de Ubajara, Trilha Araticum, Rio das Minas na altura da trilha do teleférico; maximumElevationInMeters: 420; verbatimCoordinates: 349'58"S, 4053'53"W; samplingProtocol: Malaise intercept trap; verbatimEventDate: 20.iv.12; individualCount: 1; sex: male; lifeStage: adult; recordedBy: Rafael, J.A. | Limeira-de-Oliveira, F. | Takiya, D.M. | et al.; identifiedBy: Allan Paulo Moreira dos Santos; institutionCode: DZRJ; basisOfRecord: PreservedSpecimen

n. country: Brazil; stateProvince: Ceará; municipality: Ubajara; locality: Parque Nacional de Ubajara, Trilha Araticum, Rio das Minas na altura da trilha do teleférico; maximumElevationInMeters: 420; verbatimCoordinates: $3^{\circ} 49^{\prime} 58^{\prime \prime S}, 40^{\circ} 53^{\prime} 53^{\prime \prime} \mathrm{W}$; samplingProtocol: Malaise intercept trap; verbatimEventDate: 20.iv.12; individualCount: 3; sex: male; lifeStage: adult; recordedBy: Rafael, J.A. | Limeira-de-Oliveira, F. | Takiya, D.M. | et al.; identifiedBy: Allan Paulo Moreira dos Santos; institutionCode: DZRJ; basisOfRecord: PreservedSpecimen

o. country: Brazil; stateProvince: Ceará; municipality: Ubajara; locality: Parque Nacional de Ubajara, Trilha Araticum, Rio das Minas na altura da trilha do teleférico; maximumElevationInMeters: 420; verbatimCoordinates: $3^{\circ} 49^{\prime} 58^{\prime \prime S}$, 4053'53"W; samplingProtocol: Malaise intercept trap; verbatimEventDate: 20.iv.12; individualCount: 1; sex: female; lifeStage: adult; recordedBy: Rafael, J.A. | Limeira-de-Oliveira, F. | Takiya, D.M. | et al.; identifiedBy: Allan Paulo Moreira dos Santos; institutionCode: DZRJ; basisOfRecord: PreservedSpecimen

p. country: Brazil; stateProvince: Ceará; municipality: Ubajara; locality: Parque Nacional de Ubajara, Trilha Araticum, Rio das Minas na altura da trilha do teleférico; maximumElevationInMeters: 420; verbatimCoordinates: $3^{\circ} 49^{\prime} 58^{\prime \prime S}, 40^{\circ} 53^{\prime} 53^{\prime \prime} \mathrm{W}$; samplingProtocol: Malaise intercept trap; verbatimEventDate: 20.iv.12; individualCount: 7; sex: male; lifeStage: adult; recordedBy: Rafael, J.A. | Limeira-de-Oliveira, F. | Takiya, D.M. | et al.; identifiedBy: Allan Paulo Moreira dos Santos; institutionCode: DZRJ; basisOfRecord: PreservedSpecimen

Distribution: Brazil: CE, MT, RO, GO, MG, ES, RJ, SC. 


\section{Chimarra (Curgia) cultellata Flint, 1983}

\section{Materials}

a. country: Brazil; stateProvince: Ceará; municipality: Ubajara; locality: Parque Nacional de Ubajara, Trilha Araticum, Rio das Minas na altura da trilha do teleférico; maximumElevationInMeters: 420 ; verbatimCoordinates: $3^{\circ} 49^{\prime} 58^{\prime \prime S}, 40^{\circ} 53^{\prime} 53^{\prime \prime} \mathrm{W}$; samplingProtocol: Malaise intercept trap; verbatimEventDate: 20.iv.12; individualCount: 1; sex: male; lifeStage: adult; recordedBy: Rafael, J.A. | Limeira-de-Oliveira, F. | Takiya, D.M. | et al.; identifiedBy: Allan Paulo Moreira dos Santos; institutionCode: DZRJ; basisOfRecord: PreservedSpecimen

b. country: Brazil; stateProvince: Ceará; municipality: Ubajara; locality: Parque Nacional de Ubajara, Trilha Araticum, Rio das Minas na altura da trilha do teleférico; maximumElevationInMeters: 420; verbatimCoordinates: $3^{\circ} 49^{\prime} 58^{\prime \prime} \mathrm{S}, 40^{\circ} 53^{\prime} 53^{\prime \prime} \mathrm{W}$;

samplingProtocol: Malaise intercept trap; verbatimEventDate: 20.iv.12; individualCount: 1; sex: male; lifeStage: adult; recordedBy: Rafael, J.A. | Limeira-de-Oliveira, F. | Takiya, D.M. | et al.; identifiedBy: Allan Paulo Moreira dos Santos; institutionCode: DZRJ; basisOfRecord: PreservedSpecimen

c. country: Brazil; stateProvince: Ceará; municipality: Ubajara; locality: Parque Nacional de Ubajara, Trilha Araticum, Rio das Minas; maximumElevationInMeters: 524; verbatimCoordinates: $3^{\circ} 50^{\prime} 3$ "S, $40^{\circ} 54^{\prime} 18^{\prime \prime} \mathrm{W}$; samplingProtocol: Pennsylvania light trap; verbatimEventDate: 22.iv.12; individualCount: 3; sex: male; lifeStage: adult; recordedBy: Takiya, D.M. | Somavilla, A.; identifiedBy: Allan Paulo Moreira dos Santos; institutionCode: DZRJ; basisOfRecord: PreservedSpecimen

d. country: Brazil; stateProvince: Ceará; municipality: Ubajara; locality: Parque Nacional de Ubajara, Trilha Araticum, Rio das Minas; maximumElevationInMeters: 524; verbatimCoordinates: $3^{\circ} 50^{\prime} 3$ "S, $40^{\circ} 54^{\prime} 18 \mathrm{~W}$; samplingProtocol: Pennsylvania light trap; verbatimEventDate: 22.iv.12; individualCount: 2; sex: female; lifeStage: adult; recordedBy: Takiya, D.M. | Somavilla, A.; identifiedBy: Allan Paulo Moreira dos Santos; institutionCode: DZRJ; basisOfRecord: PreservedSpecimen

e. $\quad$ country: Brazil; stateProvince: Ceará; municipality: Ubajara; locality: Parque Nacional de Ubajara, Rio Cafundó, pouco acima da cachoeira; maximumElevationInMeters: 795; verbatimCoordinates: $3^{\circ} 50^{\prime} 13^{\prime \prime} \mathrm{S}, 40^{\circ} 54^{\prime} 35 " \mathrm{~W}$; samplingProtocol: Pennsylvania light trap; verbatimEventDate: 24.iv.12; individualCount: 1; sex: male; lifeStage: adult; recordedBy: Takiya, D.M. | Rafael, J.A.; identifiedBy: Allan Paulo Moreira dos Santos; institutionCode: DZRJ; basisOfRecord: PreservedSpecimen

f. country: Brazil; stateProvince: Ceará; municipality: Ubajara; locality: Parque Nacional de Ubajara, Rio das Minas, próximo ao Portão Araticum; maximumElevationInMeters: 328; verbatimCoordinates: $3^{\circ} 49^{\prime} 32.6$ "S, 4053'32.8"W; samplingProtocol: Malaise intercept trap; verbatimEventDate: 14.ii.13; individualCount: 12; sex: male; lifeStage: adult; recordedBy: Rafael, J.A. | Limeira-de-Oliveira, F. | Takiya, D.M. | Santos, A.P.M. | et al.; identifiedBy: Allan Paulo Moreira dos Santos; institutionCode: DZRJ; basisOfRecord: PreservedSpecimen

g. country: Brazil; stateProvince: Ceará; municipality: Ubajara; locality: Parque Nacional de Ubajara, Rio das Minas, próximo ao Portão Araticum; maximumElevationInMeters: 328; verbatimCoordinates: $3^{\circ} 49^{\prime} 32.6$ "S, $40^{\circ} 53^{\prime} 32.8^{\prime \prime W}$; samplingProtocol: Malaise intercept trap; verbatimEventDate: 14.ii.13; individualCount: 39; sex: female; lifeStage: adult; recordedBy: Rafael, J.A. | Limeira-de-Oliveira, F. | Takiya, D.M. | Santos, A.P.M. | et al.; identifiedBy: Allan Paulo Moreira dos Santos; institutionCode: DZRJ; basisOfRecord: PreservedSpecimen 
h. country: Brazil; stateProvince: Ceará; municipality: Ubajara; locality: Parque Nacional de Ubajara, Rio das Minas, próximo ao Portão Araticum; maximumElevationInMeters: 328; verbatimCoordinates: $3^{\circ} 49^{\prime} 32.6^{\prime \prime S}, 40^{\circ} 53^{\prime} 32.8^{\prime \prime W}$; sampling Protocol: Malaise intercept trap; verbatimEventDate: 14.ii.13; individualCount: 19; sex: male; lifeStage: adult; recordedBy: Rafael, J.A. | Limeira-de-Oliveira, F. | Takiya, D.M. | Santos, A.P.M. | et al.; identifiedBy: Allan Paulo Moreira dos Santos; institutionCode: DZRJ; basisOfRecord: PreservedSpecimen

i. country: Brazil; stateProvince: Ceará; municipality: Ubajara; locality: Parque Nacional de Ubajara, Rio das Minas, próximo ao Portão Araticum; maximumElevationInMeters: 328; verbatimCoordinates: $3^{\circ} 49^{\prime} 32.6^{\prime \prime S}, 40^{\circ} 53^{\prime} 32.8^{\prime \prime W}$; sampling Protocol: Malaise intercept trap; verbatimEventDate: 14.ii.13; individualCount: 37; sex: female; lifeStage: adult; recordedBy: Rafael, J.A. | Limeira-de-Oliveira, F. | Takiya, D.M. | Santos, A.P.M. | et al.; identifiedBy: Allan Paulo Moreira dos Santos; institutionCode: DZRJ; basisOfRecord: PreservedSpecimen

j. country: Brazil; stateProvince: Ceará; municipality: Ubajara; locality: Parque Nacional de Ubajara, Trilha Araticum, Rio das Minas; maximumElevationInMeters: 524; verbatimCoordinates: $3^{\circ} 50^{\prime} 3$ "S, $40^{\circ} 54^{\prime} 18 \mathrm{~W}$; samplingProtocol: Malaise intercept trap; verbatimEventDate: 17.ii.13; individualCount: 2; sex: male; lifeStage: adult; recordedBy: Rafael, J.A. | Limeira-de-Oliveira, F. | Takiya, D.M. | Santos, A.P.M. | et al.; identifiedBy: Allan Paulo Moreira dos Santos; institutionCode: DZRJ; basisOfRecord: PreservedSpecimen

k. country: Brazil; stateProvince: Ceará; municipality: Ubajara; locality: Parque Nacional de Ubajara, Rio das Minas, próximo ao Portão Araticum; maximumElevationInMeters: 328; verbatimCoordinates: $3^{\circ} 49^{\prime} 32.6$ "S, 4053'32.8"W; samplingProtocol: Malaise intercept trap; verbatimEventDate: 17.ii.13; individualCount: 19; sex: male; lifeStage: adult; recordedBy: Rafael, J.A. | Limeira-de-Oliveira, F. | Takiya, D.M. | Santos, A.P.M. | et al.; identifiedBy: Allan Paulo Moreira dos Santos; institutionCode: DZRJ; basisOfRecord: PreservedSpecimen

I. country: Brazil; stateProvince: Ceará; municipality: Ubajara; locality: Parque Nacional de Ubajara, Rio das Minas, próximo ao Portão Araticum; maximumElevationInMeters: 328; verbatimCoordinates: $3^{\circ} 49^{\prime} 32.6^{\prime \prime}$ S, 4053'32.8"W; samplingProtocol: Malaise intercept trap; verbatimEventDate: 17.ii.13; individualCount: 13; sex: female; lifeStage: adult; recordedBy: Rafael, J.A. | Limeira-de-Oliveira, F. | Takiya, D.M. | Santos, A.P.M. | et al.; identifiedBy: Allan Paulo Moreira dos Santos; institutionCode: DZRJ; basisOfRecord: PreservedSpecimen

m. country: Brazil; stateProvince: Ceará; municipality: Ubajara; locality: Parque Nacional de Ubajara, Trilha Araticum, Rio das Minas na altura da trilha do teleférico; maximumElevationInMeters: 420; verbatimCoordinates: $3^{\circ} 49^{\prime} 58^{\prime \prime S}, 40^{\circ} 53^{\prime} 53 " \mathrm{~W}$; samplingProtocol: Malaise intercept trap; verbatimEventDate: 20.iv.12; individualCount: 1; sex: male; lifeStage: adult; recordedBy: Rafael, J.A. | Limeira-de-Oliveira, F. | Takiya, D.M. I et al.; identifiedBy: Allan Paulo Moreira dos Santos; institutionCode: DZRJ; basisOfRecord: PreservedSpecimen

n. country: Brazil; stateProvince: Ceará; municipality: Ubajara; locality: Parque Nacional de Ubajara, Trilha Araticum, Rio das Minas na altura da trilha do teleférico; maximumElevationInMeters: 420; verbatimCoordinates: $3^{\circ} 49^{\prime} 58^{\prime \prime S}, 40^{\circ} 53^{\prime} 53 " \mathrm{~W}$; samplingProtocol: Malaise intercept trap; verbatimEventDate: 20.iv.12; individualCount: 3; sex: male; lifeStage: adult; recordedBy: Rafael, J.A. | Limeira-de-Oliveira, F. | Takiya, D.M. I et al.; identifiedBy: Allan Paulo Moreira dos Santos; institutionCode: DZRJ; basisOfRecord: PreservedSpecimen 
o. country: Brazil; stateProvince: Ceará; municipality: Ubajara; locality: Parque Nacional de Ubajara, Trilha Araticum, Rio das Minas na altura da trilha do teleférico; maximumElevationInMeters: 420; verbatimCoordinates: $3^{\circ} 49^{\prime} 58^{\prime \prime S}, 40^{\circ} 53^{\prime} 53^{\prime \prime} \mathrm{W}$; samplingProtocol: Malaise intercept trap; verbatimEventDate: 20.iv.12; individualCount: 1; sex: female; lifeStage: adult; recordedBy: Rafael, J.A. | Limeira-de-Oliveira, F. | Takiya, D.M. | et al.; identifiedBy: Allan Paulo Moreira dos Santos; institutionCode: DZRJ; basisOfRecord: PreservedSpecimen

p. country: Brazil; stateProvince: Ceará; municipality: Ubajara; locality: Parque Nacional de Ubajara, Trilha Araticum, Rio das Minas na altura da trilha do teleférico; maximumElevationInMeters: 420; verbatimCoordinates: $3^{\circ} 49^{\prime} 58^{\prime \prime S}, 40^{\circ} 53^{\prime} 53 " \mathrm{~W}$; samplingProtocol: Malaise intercept trap; verbatimEventDate: 20.iv.12; individualCount: 7; sex: male; lifeStage: adult; recordedBy: Rafael, J.A. | Limeira-de-Oliveira, F. | Takiya, D.M. | et al.; identifiedBy: Allan Paulo Moreira dos Santos; institutionCode: DZRJ; basisOfRecord: PreservedSpecimen

Distribution: Brazil: PA, AM, CE!, RO, MG, DF, RJ, SC.

Notes: New species record for Northeastern Brazil.

\section{Family Polycentropodidae}

Notes: New family record for CE.

\section{Genus Cernotina Ross, 1938}

Notes: New genus record for CE.

\section{Cernotina sp. $2^{*}$}

\section{Materials}

a. country: Brazil; stateProvince: Ceará; municipality: Ubajara; locality: Parque Nacional de Ubajara, Trilha Samambaia, Rio Gameleira; maximumElevationInMeters: 874; verbatimCoordinates: $3^{\circ} 50^{\prime 2} 25^{\prime} \mathrm{S}, 40^{\circ} 54^{\prime} 19^{\prime \prime} \mathrm{W}$; sampling Protocol: Pennsylvania light trap; verbatimEventDate: 13.ii.13; individualCount: 1; sex: male; lifeStage: adult; recordedBy: Santos, A.P.M. | Takiya, D.M.; identifiedBy: Allan Paulo Moreira dos Santos; institutionCode: DZRJ; basisOfRecord: PreservedSpecimen

b. country: Brazil; stateProvince: Ceará; municipality: Ubajara; locality: Parque Nacional de Ubajara, Rio Cafundó, pouco acima da cachoeira; maximumElevationInMeters: 795; verbatimCoordinates: $3^{\circ} 50^{\prime} 13^{\prime \prime S}, 40^{\circ} 54^{\prime} 35 \mathrm{WW}$; samplingProtocol: Malaise intercept trap; verbatimEventDate: 13.ii.13; individualCount: 1; sex: male; lifeStage: adult; recordedBy: Rafael, J.A. | Limeira-de-Oliveira, F. | Takiya, D.M. | Santos, A.P.M. | et al.; identifiedBy: Allan Paulo Moreira dos Santos; institutionCode: DZRJ; basisOfRecord: PreservedSpecimen

c. country: Brazil; stateProvince: Ceará; municipality: Ubajara; locality: Parque Nacional de Ubajara, Trilha Samambaia, Rio Gameleira; maximumElevationInMeters: 874; verbatimCoordinates: $3^{\circ} 50^{\prime} 25 " \mathrm{~S}, 40^{\circ} 54^{\prime} 19 " \mathrm{~W}$; samplingProtocol: Pennsylvania light trap; verbatimEventDate: 14.ii.13; individualCount: 1; sex: male; lifeStage: adult; recordedBy: Santos, A.P.M. | Takiya, D.M.; identifiedBy: Allan Paulo Moreira dos Santos; institutionCode: DZRJ; basisOfRecord: PreservedSpecimen 
d. country: Brazil; stateProvince: Ceará; municipality: Ubajara; locality: Parque Nacional de Ubajara, Trilha Samambaia, Rio Gameleira; maximumElevationInMeters: 874; verbatimCoordinates: $3^{\circ} 50^{\prime} 25^{\prime \prime S}, 40^{\circ} 54^{\prime} 19 " \mathrm{~W}$; samplingProtocol: Malaise intercept trap; verbatimEventDate: 17.ii.13; individualCount: 1; sex: male; lifeStage: adult; recordedBy: Rafael, J.A. | Limeira-de-Oliveira, F. | Takiya, D.M. | Santos, A.P.M. | et al.; identifiedBy: Allan Paulo Moreira dos Santos; institutionCode: DZRJ; basisOfRecord: PreservedSpecimen

e. $\quad$ country: Brazil; stateProvince: Ceará; municipality: Ubajara; locality: Parque Nacional de Ubajara, Trilha Araticum, Rio das Minas; maximumElevationInMeters: 524; verbatimCoordinates: $3^{\circ} 50^{\prime} 3$ "S, $40^{\circ} 54^{\prime} 18^{\prime \prime W}$; samplingProtocol: Malaise intercept trap; verbatimEventDate: 17.ii.13; individualCount: 1; sex: male; lifeStage: adult; recordedBy: Rafael, J.A. | Limeira-de-Oliveira, F. | Takiya, D.M. | Santos, A.P.M. | et al.; identifiedBy: Allan Paulo Moreira dos Santos; institutionCode: DZRJ; basisOfRecord: PreservedSpecimen

f. country: Brazil; stateProvince: Ceará; municipality: Ubajara; locality: Parque Nacional de Ubajara, Trilha Araticum, Rio das Minas na altura da trilha do teleférico; maximumElevationInMeters: 420; verbatimCoordinates: $3^{\circ} 49^{\prime} 58^{\prime \prime S}, 40^{\circ} 53^{\prime} 53^{\prime \prime} \mathrm{W}$; samplingProtocol: Malaise intercept trap; verbatimEventDate: 20.iv.12; individualCount: 2; sex: male; lifeStage: adult; recordedBy: Rafael, J.A. | Limeira-de-Oliveira, F. | Takiya, D.M. I et al.; identifiedBy: Allan Paulo Moreira dos Santos; institutionCode: DZRJ; basisOfRecord: PreservedSpecimen

g. country: Brazil; stateProvince: Ceará; municipality: Ubajara; locality: Parque Nacional de Ubajara, Trilha Araticum, Rio das Minas na altura da trilha do teleférico; maximumElevationInMeters: 420; verbatimCoordinates: $3^{\circ} 49^{\prime} 58^{\prime \prime S}, 40^{\circ} 53^{\prime} 53^{\prime \prime} \mathrm{W}$; samplingProtocol: Malaise intercept trap; verbatimEventDate: 20.iv.12; individualCount: 1; sex: female; lifeStage: adult; recordedBy: Rafael, J.A. | Limeira-de-Oliveira, F. | Takiya, D.M. | et al.; identifiedBy: Allan Paulo Moreira dos Santos; institutionCode: DZRJ; basisOfRecord: PreservedSpecimen

h. country: Brazil; stateProvince: Ceará; municipality: Ubajara; locality: Parque Nacional de Ubajara, Trilha Araticum, Rio das Minas na altura da trilha do teleférico; maximumElevationInMeters: 420; verbatimCoordinates: $3^{\circ} 49^{\prime} 58^{\prime \prime S}, 40^{\circ} 53^{\prime} 53 " \mathrm{~W}$; samplingProtocol: Malaise intercept trap; verbatimEventDate: 20.iv.12; individualCount: 2; sex: male; lifeStage: adult; recordedBy: Rafael, J.A. | Limeira-de-Oliveira, F. | Takiya, D.M. | et al.; identifiedBy: Allan Paulo Moreira dos Santos; institutionCode: DZRJ; basisOfRecord: PreservedSpecimen

i. country: Brazil; stateProvince: Ceará; municipality: Ubajara; locality: Parque Nacional de Ubajara, Rio Cafundó, pouco acima da cachoeira; maximumElevationInMeters: 795; verbatimCoordinates: $3^{\circ} 50^{\prime} 13^{\prime \prime S}, 40^{\circ} 54^{\prime} 35 \mathrm{WW}$; samplingProtocol: Malaise intercept trap; verbatimEventDate: 21.iv.12; individualCount: 10; sex: male; lifeStage: adult; recordedBy: Rafael, J.A. | Limeira-de-Oliveira, F. | Takiya, D.M. | et al.; identifiedBy: Allan Paulo Moreira dos Santos; institutionCode: DZRJ; basisOfRecord: PreservedSpecimen 


\section{Genus Polycentropus Curtis, 1835}

Notes: New genus record for CE.

\section{Polycentropus sp. 1}

\section{Material}

a. country: Brazil; stateProvince: Ceará; municipality: Ubajara; locality: Parque Nacional de Ubajara, Trilha Araticum, Rio das Minas; maximumElevationInMeters: 524; verbatimCoordinates: 350'3"S, 4054'18"W; samplingProtocol: Malaise intercept trap; verbatimEventDate: 14.ii.13; individualCount: 1; sex: male; lifeStage: adult; recordedBy: Rafael, J.A. | Limeira-de-Oliveira, F. | Takiya, D.M. | Santos, A.P.M. | et al.; identifiedBy: Allan Paulo Moreira dos Santos; institutionCode: DZRJ; basisOfRecord: PreservedSpecimen

\section{Family Xiphocentronidae}

Notes: New family record for CE.

\section{Genus Xiphocentron Brauer, 1870}

Notes: New genus record for CE.

\section{Xiphocentron sp. 1}

\section{Materials}

a. country: Brazil; stateProvince: Ceará; municipality: Ubajara; locality: Parque Nacional de Ubajara, Rio Cafundó, pouco acima da cachoeira; maximumElevationInMeters: 795; verbatimCoordinates: $3^{\circ} 50^{\prime} 13^{\prime \prime} \mathrm{S}, 40^{\circ} 54^{\prime} 35 \mathrm{WW}$; samplingProtocol: Malaise intercept trap; verbatimEventDate: 13.ii.13; individualCount: 1; sex: male; lifeStage: adult; recordedBy: Rafael, J.A. | Limeira-de-Oliveira, F. | Takiya, D.M. | Santos, A.P.M. | et al.; identifiedBy: Allan Paulo Moreira dos Santos; institutionCode: DZRJ; basisOfRecord: PreservedSpecimen

b. country: Brazil; stateProvince: Ceará; municipality: Ubajara; locality: Parque Nacional de Ubajara, Rio Cafundó, pouco acima da cachoeira; maximumElevationInMeters: 795; verbatimCoordinates: $3^{\circ} 50^{\prime} 13^{\prime \prime S}, 40^{\circ} 54^{\prime} 35 \mathrm{~W} \mathrm{~W}$; samplingProtocol: Malaise intercept trap; verbatimEventDate: 13.ii.13; individualCount: 3; sex: female; lifeStage: adult; recordedBy: Rafael, J.A. | Limeira-de-Oliveira, F. | Takiya, D.M. | Santos, A.P.M. | et al.; identifiedBy: Allan Paulo Moreira dos Santos; institutionCode: DZRJ; basisOfRecord: PreservedSpecimen

c. country: Brazil; stateProvince: Ceará; municipality: Ubajara; locality: Parque Nacional de Ubajara, Rio Cafundó, pouco acima da cachoeira; maximumElevationInMeters: 795; verbatimCoordinates: $3^{\circ} 50^{\prime} 13^{\prime \prime S}, 40^{\circ} 54^{\prime} 35 \mathrm{~W} \mathrm{~W}$; samplingProtocol: Malaise intercept trap; verbatimEventDate: 13.xi.12; individualCount: 2; sex: male; lifeStage: adult; recordedBy: Limeira-de-Oliveira | et al.; identifiedBy: Allan Paulo Moreira dos Santos; institutionCode: DZRJ; basisOfRecord: PreservedSpecimen 


\section{Aquatic insects from Parque Nacional de Sete Cidades (PNSC)}

\section{Order Coleoptera}

\section{Family Epimetopidae}

Notes: New family record for PI.

\section{Genus Epimetopus Lacordaire, 1854}

Notes: New genus record for PI.

\section{Epimetopus lacordairei Orchymont, 1933}

Material

a. country: Brazil; stateProvince: Piauí; municipality: Piracuruca; locality: Parque Nacional de Sete Cidades, Cachoeira do Riachão; maximumElevationInMeters: 171; verbatimCoordinates: $4^{\circ} 6^{\prime} 28^{\prime \prime S}, 41^{\circ} 40^{\prime} 13^{\prime \prime} \mathrm{W}$; sampling Protocol: White sheet light trap; verbatimEventDate: 18.iv.12; individualCount: 1; sex: female; lifeStage: adult; recordedBy: Takiya, D.M.; identifiedBy: Bruno Clarkson; institutionCode: DZRJ; basisOfRecord: PreservedSpecimen

Distribution: Brazil: PI!, MS. Bolivia. Paraguay

Notes: New species record for Northeastern Brazil.

\section{Family Hydrophilidae}

Genus Crenitulus Winters, 1926

Notes: New genus record for PI.

\section{Crenitulus suturalis (LeConte, 1866)}

\section{Materials}

a. country: Brazil; stateProvince: Piauí; municipality: Piracuruca; locality: Parque Nacional de Sete Cidades, Olha d'água dos Milagres; maximumElevationInMeters: 180; verbatimCoordinates: $4^{\circ} 5^{\prime} 31.8^{\prime \prime S}, 41^{\circ} 40^{\prime} 48.2^{\prime \prime} \mathrm{W}$; samplingProtocol: Pennsylvania light trap; verbatimEventDate: 15.ii.13; individualCount: 1; sex: female; lifeStage: adult; recordedBy: Santos, A.P.M. | Takiya, D.M.; identifiedBy: Bruno Clarkson; institutionCode: DZRJ; basisOfRecord: PreservedSpecimen

b. country: Brazil; stateProvince: Piauí; municipality: Piracuruca; locality: Parque Nacional de Sete Cidades, Riacho da Piedade; maximumElevationInMeters: 169; verbatimCoordinates: $4^{\circ} 6^{\prime} 34$ "S, $41^{\circ} 43^{\prime} 39 " \mathrm{~W}$; samplingProtocol: Manual; verbatimEventDate: 18.iv.12; individualCount: 1; sex: male; lifeStage: adult; recordedBy: 
Takiya, D.M.; identifiedBy: Bruno Clarkson; institutionCode: DZRJ; basisOfRecord: PreservedSpecimen

Distribution: USA. Mexico. Belize. Guatemala. Honduras. Nicaragua. Costa Rica. Dominican Republic. Jamaica. Brazil: PI!, CE, PE, AL, MS, SP, RJ, SC. Peru. Bolivia. Paraguay. Argentina.

Notes: New species record for PI.

\section{Genus Berosus Leach, 1817}

\section{Berosus patruelis Berg, 1885}

\section{Materials}

a. country: Brazil; stateProvince: Piauí; municipality: Piracuruca; locality: Parque Nacional de Sete Cidades, Cachoeira do Riachão; maximumElevationInMeters: 171; verbatimCoordinates: $4^{\circ} 6^{\prime} 28$ "S, $41^{\circ} 40^{\prime} 13^{\prime \prime} \mathrm{W}$; samplingProtocol: Pennsylvania light trap; verbatimEventDate: 18.iv.12; individualCount: 4; sex: male; lifeStage: adult; recordedBy: Takiya, D.M.; identifiedBy: Bruno Clarkson; institutionCode: DZRJ; basisOfRecord: PreservedSpecimen

b. $\quad$ country: Brazil; stateProvince: Piauí; municipality: Piracuruca; locality: Parque Nacional de Sete Cidades, Cachoeira do Riachão; maximumElevationInMeters: 171; verbatimCoordinates: $4^{\circ} 6^{\prime} 28$ "S, $41^{\circ} 40^{\prime} 13$ "W; samplingProtocol: Pennsylvania light trap; verbatimEventDate: 18.iv.12; individualCount: 1; sex: female; lifeStage: adult; recordedBy: Takiya, D.M.; identifiedBy: Bruno Clarkson; institutionCode: DZRJ; basisOfRecord: PreservedSpecimen

c. country: Brazil; stateProvince: Piauí; municipality: Piracuruca; locality: Parque Nacional de Sete Cidades, Alojamento; maximumElevationInMeters: 193; verbatimCoordinates: $4^{\circ}$ 5'57"S, 4142'34"W; samplingProtocol: White sheet light trap; verbatimEventDate: 18.iv.12; individualCount: 1; sex: male; lifeStage: adult; recordedBy: Takiya, D.M.; identifiedBy: Bruno Clarkson; institutionCode: DZRJ; basisOfRecord: PreservedSpecimen

d. country: Brazil; stateProvince: Piauí; municipality: Piracuruca; locality: Parque Nacional de Sete Cidades, Alojamento; maximumElevationInMeters: 193; verbatimCoordinates: $4^{\circ}$ 5'57"S, 4142'34"W; samplingProtocol: White sheet light trap; verbatimEventDate: 18.iv.12; individualCount: 1; sex: female; lifeStage: adult; recordedBy: Takiya, D.M.; identifiedBy: Bruno Clarkson; institutionCode: DZRJ; basisOfRecord: PreservedSpecimen

Distribution: Venezuela. Brazil: PI, PE, MS. Bolivia. Paraguay. Argentina.

\section{Berosus consobrinus Knisch, 1921}

\section{Materials}

a. country: Brazil; stateProvince: Piauí; municipality: Piracuruca; locality: Parque Nacional de Sete Cidades, Cachoeira do Riachão; maximumElevationInMeters: 171; verbatimCoordinates: $4^{\circ} 6^{\prime} 28$ "S, $41^{\circ} 40^{\prime} 13$ "W; samplingProtocol: Pennsylvania light trap; verbatimEventDate: 18.iv.12; individualCount: 2; sex: female; lifeStage: adult; recordedBy: Takiya, D.M.; identifiedBy: Bruno Clarkson; institutionCode: DZRJ; basisOfRecord: PreservedSpecimen 
b. country: Brazil; stateProvince: Piauí; municipality: Piracuruca; locality: Parque Nacional de Sete Cidades, Cachoeira do Riachão; maximumElevationInMeters: 171; verbatimCoordinates: $4^{\circ} 6^{\prime} 28$ "S, $41^{\circ} 40^{\prime} 13$ "W; samplingProtocol: Pennsylvania light trap; verbatimEventDate: 18.iv.12; individualCount: 2; sex: male; lifeStage: adult; recordedBy: Takiya, D.M.; identifiedBy: Bruno Clarkson; institutionCode: DZRJ; basisOfRecord: PreservedSpecimen

c. country: Brazil; stateProvince: Piauí; municipality: Piracuruca; locality: Parque Nacional de Sete Cidades, Alojamento; maximumElevationInMeters: 193; verbatimCoordinates: $4^{\circ}$ 5'57"S, 4142'34"W; samplingProtocol: White sheet light trap; verbatimEventDate: 18.iv.12; individualCount: 3; sex: male; lifeStage: adult; recordedBy: Takiya, D.M.; identifiedBy: Bruno Clarkson; institutionCode: DZRJ; basisOfRecord: PreservedSpecimen

d. country: Brazil; stateProvince: Piauí; municipality: Piracuruca; locality: Parque Nacional de Sete Cidades, Alojamento; maximumElevationInMeters: 193; verbatimCoordinates: $4^{\circ}$ 5'57"S, 4142'34"W; samplingProtocol: White sheet light trap; verbatimEventDate: 18.iv.12; individualCount: 2; sex: female; lifeStage: adult; recordedBy: Takiya, D.M.; identifiedBy: Bruno Clarkson; institutionCode: DZRJ; basisOfRecord: PreservedSpecimen

Distribution: Venezuela. Brazil: PI!, MS.

Notes: New species record for Northeastern Brazil.

\section{Berosus erraticus Mouchamps, 1963}

\section{Material}

a. country: Brazil; stateProvince: Piauí; municipality: Piracuruca; locality: Parque Nacional de Sete Cidades, Cachoeira do Riachão; maximumElevationInMeters: 171; verbatimCoordinates: $4^{\circ} 6^{\prime} 28^{\prime \prime S}, 41^{\circ} 40^{\prime} 13$ "W; samplingProtocol: Pennsylvania light trap; verbatimEventDate: 18.iv.12; individualCount: 1; sex: male; lifeStage: adult; recordedBy: Takiya, D.M.; identifiedBy: Bruno Clarkson; institutionCode: DZRJ; basisOfRecord: PreservedSpecimen

Distribution: Venezuela. Brazil: AM, PI!, RJ. Bolivia. Paraguay. Argentina. Uruguay.

Notes: New species record for Northeastern Brazil.

\section{Berosus festivus Berg, 1885}

\section{Materials}

a. country: Brazil; stateProvince: Piauí; municipality: Piracuruca; locality: Parque Nacional de Sete Cidades, Cachoeira do Riachão; maximumElevationInMeters: 171; verbatimCoordinates: $4^{\circ} 6^{\prime} 28^{\prime \prime} \mathrm{S}, 41^{\circ} 40^{\prime} 13^{\prime \prime} \mathrm{W}$; samplingProtocol: Manual; verbatimEventDate: 19.ii.13; individualCount: 1; sex: male; lifeStage: adult; recordedBy: Santos, A.P.M. | Takiya, D.M.; identifiedBy: Bruno Clarkson; institutionCode: DZRJ; basisOfRecord: PreservedSpecimen

b. $\quad$ country: Brazil; stateProvince: Piauí; municipality: Piracuruca; locality: Parque Nacional de Sete Cidades, Poço do Bananeira; maximumElevationInMeters: 158; verbatimCoordinates: $4^{\circ} 5^{\prime} 55.8^{\prime \prime} \mathrm{S}, 41^{\circ} 40^{\prime} 33.8^{\prime \prime} \mathrm{W}$; samplingProtocol: Manual; verbatimEventDate: 9.ii.13; individualCount: 1; sex: male; lifeStage: adult; recordedBy: 
Santos, A.P.M. | Takiya, D.M.; identifiedBy: Bruno Clarkson; institutionCode: DZRJ; basisOfRecord: PreservedSpecimen

Distribution: Venezuela. Guyana. Brazil: PI!, CE, PE, MT, MS, RJ. Argentina. Uruguay. Notes: New species record for PI.

\section{Berosus sp. 1}

\section{Material}

a. country: Brazil; stateProvince: Piauí; municipality: Piracuruca; locality: Parque Nacional de Sete Cidades, Riacho da Bananeira; maximumElevationInMeters: 189; verbatimCoordinates: $4^{\circ} 5^{\prime} 59$ "S, $41^{\circ} 40^{\prime} 48$ "W; samplingProtocol: Manual; verbatimEventDate: 11.ii.13; individualCount: 1; sex: female; lifeStage: adult; recordedBy: Santos, A.P.M. | Takiya, D.M.; identifiedBy: Bruno Clarkson; institutionCode: DZRJ; basisOfRecord: PreservedSpecimen

\section{Berosus sp. 2}

\section{Materials}

a. country: Brazil; stateProvince: Piauí; municipality: Piracuruca; locality: Parque Nacional de Sete Cidades, Cachoeira do Riachão; maximumElevationInMeters: 171; verbatimCoordinates: $4^{\circ} 6^{\prime} 28$ "S, $41^{\circ} 40^{\prime} 13^{\prime \prime} \mathrm{W}$; samplingProtocol: Pennsylvania light trap; verbatimEventDate: 19.iv.12; individualCount: 1; sex: female; lifeStage: adult; recordedBy: Takiya, D.M.; identifiedBy: Bruno Clarkson; institutionCode: DZRJ; basisOfRecord: PreservedSpecimen

b. country: Brazil; stateProvince: Piauí; municipality: Piracuruca; locality: Parque Nacional de Sete Cidades, Cachoeira do Riachão; maximumElevationInMeters: 171; verbatimCoordinates: $4^{\circ} 6^{\prime} 28^{\prime \prime} \mathrm{S}, 41^{\circ} 40^{\prime} 13^{\prime \prime} \mathrm{W}$; samplingProtocol: Pennsylvania light trap; verbatimEventDate: 19.iv.12; individualCount: 2; sex: male; lifeStage: adult; recordedBy: Takiya, D.M.; identifiedBy: Bruno Clarkson; institutionCode: DZRJ; basisOfRecord: PreservedSpecimen

c. country: Brazil; stateProvince: Piauí; municipality: Piracuruca; locality: Parque Nacional de Sete Cidades, Cachoeira do Riachão; maximumElevationInMeters: 171; verbatimCoordinates: $4^{\circ} 6^{\prime} 28^{\prime \prime} \mathrm{S}, 41^{\circ} 40^{\prime} 13^{\prime \prime} \mathrm{W}$; samplingProtocol: Pennsylvania light trap; verbatimEventDate: 19.iv.12; individualCount: 2; sex: female; lifeStage: adult; recordedBy: Takiya, D.M.; identifiedBy: Bruno Clarkson; institutionCode: DZRJ; basisOfRecord: PreservedSpecimen

d. country: Brazil; stateProvince: Piauí; municipality: Piracuruca; locality: Parque Nacional de Sete Cidades, Cachoeira do Riachão; maximumElevationInMeters: 171; verbatimCoordinates: $4^{\circ} 6^{\prime} 28$ "S, $41^{\circ} 40^{\prime} 13$ "W; samplingProtocol: Pennsylvania light trap; verbatimEventDate: 19.iv.12; individualCount: 1; sex: male; lifeStage: adult; recordedBy: Takiya, D.M.; identifiedBy: Bruno Clarkson; institutionCode: DZRJ; basisOfRecord: PreservedSpecimen

Notes: Undescribed species. 


\section{Genus Chasmogenus Sharp, 1882}

Notes: New genus record for PI.

\section{Chasmogenus (Chasmogenus) sp. 1*}

\section{Materials}

a. country: Brazil; stateProvince: Piauí; municipality: Piracuruca; locality: Parque Nacional de Sete Cidades, Cachoeira do Riachão; maximumElevationInMeters: 171; verbatimCoordinates: $4^{\circ} 6^{\prime} 28$ "S, $41^{\circ} 40^{\prime} 13^{\prime \prime} \mathrm{W}$; samplingProtocol: Pennsylvania light trap; verbatimEventDate: 18.iv.12; individualCount: 1; lifeStage: adult; recordedBy: Takiya, D.M.; identifiedBy: Bruno Clarkson; institutionCode: DZRJ; basisOfRecord: PreservedSpecimen

b. country: Brazil; stateProvince: Piauí; municipality: Piracuruca; locality: Parque Nacional de Sete Cidades, Cachoeira do Riachão; maximumElevationInMeters: 171; verbatimCoordinates: $4^{\circ} 6^{\prime} 28^{\prime \prime} \mathrm{S}, 41^{\circ} 40^{\prime} 13^{\prime \prime} \mathrm{W}$; samplingProtocol: Pennsylvania light trap; verbatimEventDate: 20.iv.12; individualCount: 2; lifeStage: adult; recordedBy: Takiya, D.M.; identifiedBy: Bruno Clarkson; institutionCode: DZRJ; basisOfRecord: PreservedSpecimen

\section{Genus Enochrus Thomson, 1859}

\section{Enochrus (Methydrus) atlantis Orchymont, 1943}

\section{Material}

a. country: Brazil; stateProvince: Piauí; municipality: Piracuruca; locality: Parque Nacional de Sete Cidades, Alojamento; maximumElevationInMeters: 193; verbatimCoordinates: $4^{\circ}$ 5'57"S, 4142'34"W; samplingProtocol: White sheet light trap; verbatimEventDate: 8.11.13; individualCount: 1; sex: male; lifeStage: adult; recordedBy: Santos, A.P.M. | Takiya, D.M.; identifiedBy: Bruno Clarkson; institutionCode: DZRJ; basisOfRecord: PreservedSpecimen

Distribution: Brazil: PI, PE, MS, SP, RJ.

\section{Enochrus (Methydrus) sp. 1}

\section{Material}

a. country: Brazil; stateProvince: Piauí; municipality: Piracuruca; locality: Parque Nacional de Sete Cidades, Olha d'água dos Milagres; maximumElevationInMeters: 180; verbatimCoordinates: $4^{\circ} 5^{\prime} 31.8^{\prime \prime} \mathrm{S}, 41^{\circ} 40^{\prime} 48.2^{\prime \prime} \mathrm{W}$; samplingProtocol: Manual; verbatimEventDate: 9.11.13; individualCount: 1; sex: male; lifeStage: adult; recordedBy: Santos, A.P.M. | Takiya, D.M.; identifiedBy: Bruno Clarkson; institutionCode: DZRJ; basisOfRecord: PreservedSpecimen 


\section{Genus Helochares Mulsant, 1844}

Notes: New genus record for PI.

\section{Helochares (Helochares) tectiformis Fernández 1982}

\section{Materials}

a. country: Brazil; stateProvince: Piauí; municipality: Piracuruca; locality: Parque Nacional de Sete Cidades, Cachoeira do Riachão; maximumElevationInMeters: 171; verbatimCoordinates: $4^{\circ} 6^{\prime} 28^{\prime \prime S}, 4^{\circ} 40^{\prime} 13^{\prime \prime} \mathrm{W}$; sampling Protocol: Pennsylvania light trap; verbatimEventDate: 10.ii.13; individualCount: 1; sex: female; lifeStage: adult; recordedBy: Santos, A.P.M. | Takiya, D.M.; identifiedBy: Bruno Clarkson; institutionCode: DZRJ; basisOfRecord: PreservedSpecimen

b. country: Brazil; stateProvince: Piauí; municipality: Piracuruca; locality: Parque Nacional de Sete Cidades, Cachoeira do Riachão; maximumElevationInMeters: 171;

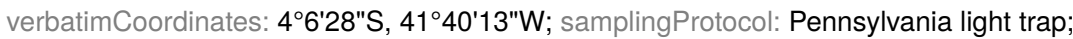
verbatimEventDate: 18.iv.12; individualCount: 1; sex: male; lifeStage: adult; recordedBy: Takiya, D.M.; identifiedBy: Bruno Clarkson; institutionCode: DZRJ; basisOfRecord: PreservedSpecimen

Distribution: Venezuela. Brazil: PI!, MS. Paraguay. Argentina.

Notes: New species record for Northeastern Brazil.

\section{Helochares (Helochares) sp. 1*}

\section{Material}

a. $\quad$ country: Brazil; stateProvince: Piauí; municipality: Piracuruca; locality: Parque Nacional de Sete Cidades, Alojamento; maximumElevationInMeters: 193; verbatimCoordinates: $4^{\circ}$ 5'57"S, 4142'34"W; sampling Protocol: White sheet light trap; verbatimEventDate: 18.iv.12; individualCount: 2; sex: female; lifeStage: adult; recordedBy: Takiya, D.M.; identifiedBy: Bruno Clarkson; institutionCode: DZRJ; basisOfRecord: PreservedSpecimen

\section{Genus Hemiosus Sharp, 1882}

\section{Hemiosus varidius Orchymont, 1940}

\section{Materials}

a. $\quad$ country: Brazil; stateProvince: Piauí; municipality: Piracuruca; locality: Parque Nacional de Sete Cidades, Cachoeira do Riachão; maximumElevationInMeters: 171; verbatimCoordinates: 46'28"S, 4140'13"W; samplingProtocol: Manual; verbatimEventDate: 19.ii.13; individualCount: 2; sex: male; lifeStage: adult; recordedBy: Santos, A.P.M. | Takiya, D.M.; identifiedBy: Bruno Clarkson; institutionCode: DZRJ; basisOfRecord: PreservedSpecimen

b. $\quad$ country: Brazil; stateProvince: Piauí; municipality: Piracuruca; locality: Parque Nacional de Sete Cidades, Cachoeira do Riachão; maximumElevationInMeters: 171; verbatimCoordinates: $4^{\circ} 6^{\prime} 28^{\prime \prime S}, 41^{\circ} 40^{\prime} 13^{\prime \prime} \mathrm{W}$; sampling Protocol: Manual; 
verbatimEventDate: 19.ii.13; individualCount: 2; sex: female; lifeStage: adult; recordedBy: Santos, A.P.M. | Takiya, D.M.; identifiedBy: Bruno Clarkson; institutionCode: DZRJ; basisOfRecord: PreservedSpecimen

c. country: Brazil; stateProvince: Piauí; municipality: Piracuruca; locality: Parque Nacional de Sete Cidades, Cachoeira do Riachão; maximumElevationInMeters: 171; verbatimCoordinates: $4^{\circ} 6^{\prime} 28^{\prime \prime S}, 41^{\circ} 40^{\prime} 13^{\prime \prime} \mathrm{W}$; samplingProtocol: Manual; verbatimEventDate: 19.ii.13; individualCount: 1; lifeStage: immature; recordedBy: Santos, A.P.M. | Takiya, D.M.; identifiedBy: Bruno Clarkson; institutionCode: DZRJ; basisOfRecord: PreservedSpecimen

d. country: Brazil; stateProvince: Piauí; municipality: Piracuruca; locality: Parque Nacional de Sete Cidades, Cachoeira do Riachão; maximumElevationInMeters: 171; verbatimCoordinates: $4^{\circ} 6^{\prime} 28$ "S, 4140'13"W; samplingProtocol: Pennsylvania light trap; verbatimEventDate: 19.iv.12; individualCount: 6; sex: male; lifeStage: adult; recordedBy: Takiya, D.M.; identifiedBy: Bruno Clarkson; institutionCode: DZRJ; basisOfRecord: PreservedSpecimen

e. country: Brazil; stateProvince: Piauí; municipality: Piracuruca; locality: Parque Nacional de Sete Cidades, Cachoeira do Riachão; maximumElevationInMeters: 171; verbatimCoordinates: $4^{\circ} 6^{\prime} 28$ "S, $41^{\circ} 40^{\prime} 13$ "W; samplingProtocol: Pennsylvania light trap; verbatimEventDate: 19.iv.12; individualCount: 9; sex: female; lifeStage: adult; recordedBy: Takiya, D.M.; identifiedBy: Bruno Clarkson; institutionCode: DZRJ; basisOfRecord: PreservedSpecimen

f. country: Brazil; stateProvince: Piauí; municipality: Piracuruca; locality: Parque Nacional de Sete Cidades, Cachoeira do Riachão; maximumElevationInMeters: 171; verbatimCoordinates: $4^{\circ} 6^{\prime} 28^{\prime \prime S}, 41^{\circ} 40^{\prime} 13$ "W; samplingProtocol: Pennsylvania light trap; verbatimEventDate: 19.iv.12; individualCount: 1; sex: male; lifeStage: adult; recordedBy: Takiya, D.M.; identifiedBy: Bruno Clarkson; institutionCode: DZRJ; basisOfRecord: PreservedSpecimen

Distribution: Brazil: PI!, PE.

Notes: New species record for PI.

\section{Hemiosus sp. 1}

\section{Materials}

a. country: Brazil; stateProvince: Piauí; municipality: Piracuruca; locality: Parque Nacional de Sete Cidades, Cachoeira do Riachão; maximumElevationInMeters: 171; verbatimCoordinates: $4^{\circ} 6^{\prime} 28$ "S, $41^{\circ} 40^{\prime} 13$ "W; samplingProtocol: Pennsylvania light trap; verbatimEventDate: 18.iv.12; individualCount: 1; sex: male; lifeStage: adult; recordedBy: Takiya, D.M.; identifiedBy: Bruno Clarkson; institutionCode: DZRJ; basisOfRecord: PreservedSpecimen

b. $\quad$ country: Brazil; stateProvince: Piauí; municipality: Piracuruca; locality: Parque Nacional de Sete Cidades, Cachoeira do Riachão; maximumElevationInMeters: 171; verbatimCoordinates: $4^{\circ} 6^{\prime} 28^{\prime \prime S}, 41^{\circ} 40^{\prime} 13^{\prime \prime W}$; samplingProtocol: Pennsylvania light trap; verbatimEventDate: 19.iv.12; individualCount: 3; sex: female; lifeStage: adult; recordedBy: Takiya, D.M.; identifiedBy: Bruno Clarkson; institutionCode: DZRJ; basisOfRecord: PreservedSpecimen

c. country: Brazil; stateProvince: Piauí; municipality: Piracuruca; locality: Parque Nacional de Sete Cidades, Olha d'água dos Milagres; maximumElevationInMeters: 180; 
verbatimCoordinates: $4^{\circ} 5^{\prime} 31.8^{\prime \prime} \mathrm{S}, 4^{\circ} 40^{\prime} 48.2^{\prime \prime W}$; samplingProtocol: Manual; verbatimEventDate: 11.ii.13; individualCount: 1; sex: female; lifeStage: adult; recordedBy: Santos, A.P.M. | Takiya, D.M.; identifiedBy: Bruno Clarkson; institutionCode: DZRJ; basisOfRecord: PreservedSpecimen

\section{Genus Oocyclus Sharp, 1882}

Notes: New genus record for PI.

\section{Oocyclus schubarti* Orchymont, 1940}

\section{Material}

a. country: Brazil; stateProvince: Piauí; municipality: Piracuruca; locality: Parque Nacional de Sete Cidades, Cachoeira do Riachão; maximumElevationInMeters: 171; verbatimCoordinates: $4^{\circ} 6^{\prime} 28$ "S, $41^{\circ} 40^{\prime} 13^{\prime \prime} \mathrm{W}$; samplingProtocol: Manual; verbatimEventDate: 9.ii.13; individualCount: 1; sex: female; lifeStage: adult; recordedBy: Santos, A.P.M. | Takiya, D.M.; identifiedBy: Bruno Clarkson; institutionCode: DZRJ; basisOfRecord: PreservedSpecimen

Distribution: Brazil: PI!, CE!, AL. Argentina?

Notes: New species record for PI.

\section{Genus Paracymus Thomson, 1867}

\section{Paracymus gracilis Orchymont, 1942}

\section{Materials}

a. country: Brazil; stateProvince: Piauí; municipality: Piracuruca; locality: Parque Nacional de Sete Cidades, Olho d'água Piscina do Bacuri; maximumElevationInMeters: 171; verbatimCoordinates: $4^{\circ} 6^{\prime} 1.2^{\prime \prime} \mathrm{S}, 4^{\circ} 42^{\prime} 38.8^{\prime \prime} \mathrm{W}$; sampling Protocol: White sheet light trap; verbatimEventDate: 11.ii.13; individualCount: 1; sex: male; lifeStage: adult; recordedBy: Santos, A.P.M. | Takiya, D.M.; identifiedBy: Bruno Clarkson; institutionCode: DZRJ; basisOfRecord: PreservedSpecimen

b. $\quad$ country: Brazil; stateProvince: Piauí; municipality: Piracuruca; locality: Parque Nacional de Sete Cidades, Olho d'água Piscina do Bacuri; maximumElevationInMeters: 171; verbatimCoordinates: $4^{\circ} 6^{\prime} 1.2^{\prime \prime} \mathrm{S}, 41^{\circ} 42^{\prime} 38.8^{\prime \prime} \mathrm{W}$; samplingProtocol: White sheet light trap; verbatimEventDate: 11.ii.13; individualCount: 3; sex: female; lifeStage: adult; recordedBy: Santos, A.P.M. | Takiya, D.M.; identifiedBy: Bruno Clarkson; institutionCode: DZRJ; basisOfRecord: PreservedSpecimen

c. country: Brazil; stateProvince: Piauí; municipality: Piracuruca; locality: Parque Nacional de Sete Cidades, Poço do Bananeira; maximumElevationInMeters: 158; verbatimCoordinates: $4^{\circ} 5^{\prime} 55.8^{\prime \prime} \mathrm{S}, 4^{\circ} 40^{\prime} 33.8^{\prime \prime} \mathrm{W}$; sampling Protocol: Pennsylvania light trap; verbatimEventDate: 11.ii.13; individualCount: 1; sex: male; lifeStage: adult; recordedBy: Santos, A.P.M. | Takiya, D.M.; identifiedBy: Bruno Clarkson; institutionCode: DZRJ; basis OfRecord: PreservedSpecimen 
d. country: Brazil; stateProvince: Piauí; municipality: Piracuruca; locality: Parque Nacional de Sete Cidades, Cachoeira do Riachão; maximumElevationInMeters: 171; verbatimCoordinates: $4^{\circ} 6^{\prime} 28$ "S, $41^{\circ} 40^{\prime} 13$ "W; samplingProtocol: Pennsylvania light trap; verbatimEventDate: 18.iv.12; individualCount: 1; sex: male; lifeStage: adult; recordedBy: Takiya, D.M.; identifiedBy: Bruno Clarkson; institutionCode: DZRJ; basisOfRecord: PreservedSpecimen

e. country: Brazil; stateProvince: Piauí; municipality: Piracuruca; locality: Parque Nacional de Sete Cidades, Cachoeira do Riachão; maximumElevationInMeters: 171; verbatimCoordinates: $4^{\circ} 6^{\prime} 28^{\prime \prime S}, 41^{\circ} 40^{\prime} 13 " \mathrm{~W}$; samplingProtocol: Pennsylvania light trap; verbatimEventDate: 18.iv.12; individualCount: 2; sex: female; lifeStage: adult; recordedBy: Takiya, D.M.; identifiedBy: Bruno Clarkson; institutionCode: DZRJ; basisOfRecord: PreservedSpecimen

Distribution: Brazil: PI!, PE, SP. Paraguay.

Notes: New species record for PI.

\section{Paracymus limbatus Wooldridge, 1973}

\section{Material}

a. country: Brazil; stateProvince: Piauí; municipality: Piracuruca; locality: Parque Nacional de Sete Cidades, Cachoeira do Riachão; maximumElevationInMeters: 171; verbatimCoordinates: $4^{\circ} 6^{\prime} 28$ "S, 41 $4{ }^{\circ} 40^{\prime} 13 " \mathrm{~W}$; samplingProtocol: Pennsylvania light trap; verbatimEventDate: 10.ii.13; individualCount: 1; sex: male; lifeStage: adult; recordedBy: Santos, A.P.M. | Takiya, D.M.; identifiedBy: Bruno Clarkson; institutionCode: DZRJ; basisOfRecord: PreservedSpecimen

Distribution: Trinidad and Tobago. Colombia. Venezuela. Suriname. Brazil: PA, PI!, RN, BA, MT, SP. Peru. Bolivia. Paraguay.

Notes: New species record for PI.

\section{Genus Tropisternus Solier, 1834}

\section{Tropisternus (Strepitornus) collaris Fabricius, 1775}

\section{Material}

a. country: Brazil; stateProvince: Piauí; municipality: Piracuruca; locality: Parque Nacional de Sete Cidades, Riacho da Bananeira; maximumElevationInMeters: 189; verbatimCoordinates: $4^{\circ} 5^{\prime} 59^{\prime \prime} \mathrm{S}, 41^{\circ} 40^{\prime} 48 \mathrm{~W} \mathrm{~W}$; samplingProtocol: Pennsylvania light trap; verbatimEventDate: 18.iv.12; individualCount: 1; sex: male; lifeStage: adult; recordedBy: Takiya, D.M.; identifiedBy: Bruno Clarkson; institutionCode: DZRJ; basisOfRecord: PreservedSpecimen

Distribution: USA. Mexico. Nicaragua. Costa Rica. Cuba. Haiti. Virgin Islands. Venezuela. Brazil: AM, CE, PI, PE, AL, SP, RJ, PR, RS. Peru. Bolivia. Paraguay. Argentina. 


\section{Tropisternus (Pristoternus) laevis (Sturm, 1826)}

\section{Material}

a. country: Brazil; stateProvince: Piauí; municipality: Piracuruca; locality: Parque Nacional de Sete Cidades, Riacho da Bananeira; maximumElevationInMeters: 189;

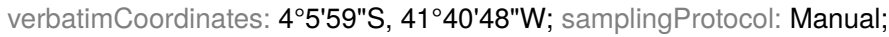
verbatimEventDate: 11.ii.13; individualCount: 1; sex: male; lifeStage: adult; recordedBy: Santos, A.P.M. | Takiya, D.M.; identifiedBy: Bruno Clarkson; institutionCode: DZRJ; basisOfRecord: PreservedSpecimen

Distribution: Venezuela. Guyana. French Guiana. Brazil: PA, AM, PI, CE, PE, AL, RJ. Paraguay. Argentina.

\section{Order Diptera}

\section{Family Empididae}

Notes: New family record for PI.

\section{Subfamily Hemerodromiinae}

Notes: New subfamily record for PI.

\section{Genus Hemerodromia Meigen, 1823}

Notes: New genus record for PI.

\section{Hemerodromia mesomelaena* Bezzi, 1909}

\section{Material}

a. country: Brazil; stateProvince: Piauí; municipality: Piracuruca; locality: Parque Nacional de Sete Cidades, Alojamento; maximumElevationInMeters: 193; verbatimCoordinates: $4^{\circ}$ 5'57"S, 4142'34"W; samplingProtocol: Malaise intercept trap; verbatimEventDate: 14.ii.13; individualCount: 7; lifeStage: adult; recordedBy: Rafael, J.A. | Limeira-de-Oliveira, F. | Takiya, D.M. | Santos, A.P.M. | et al.; identifiedBy: Josenir Teixeira Câmara; institutionCode: DZRJ; basisOfRecord: PreservedSpecimen

Distribution: Brazil: PI!, CE!, PR. Peru. Argentina.

Notes: New species record for Northeastern Brazil. 


\section{Order Ephemeroptera}

\section{Family Baetidae}

\section{Genus Callibaetis Eaton, 1881}

\section{Callibaetis itannae Cruz, Salles \& Hamada, 2014}

\section{Materials}

a. $\quad$ country: Brazil; stateProvince: Piauí; municipality: Piracuruca; locality: Parque Nacional de Sete Cidades, Cachoeira do Riachão; maximumElevationInMeters: 171;

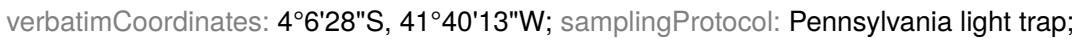
verbatimEventDate: 18.iv.12; individualCount: 11; sex: male; lifeStage: adult; recordedBy: Takiya, D.M.; identifiedBy: Inês Corrêa Gonçalves; institutionCode: DZRJ; basisOfRecord: PreservedSpecimen

b. $\quad$ country: Brazil; stateProvince: Piauí; municipality: Piracuruca; locality: Parque Nacional de Sete Cidades, Cachoeira do Riachão; maximumElevationInMeters: 171; verbatimCoordinates: $4^{\circ} 6^{\prime 2} 28^{\prime \prime S}, 41^{\circ} 40^{\prime} 13^{\prime \prime} \mathrm{W}$; samplingProtocol: Pennsylvania light trap; verbatimEventDate: 18.iv.12; individualCount: 3; sex: female; lifeStage: adult; recordedBy: Takiya, D.M.; identifiedBy: Inês Corrêa Gonçalves; institutionCode: DZRJ; basisOfRecord: PreservedSpecimen

Distribution: Brazil: PI!, RO.

Notes: New species record for Northeastern Brazil.

\section{Callibaetis nigracyclus Cruz, Salles \& Hamada, 2014}

\section{Materials}

a. country: Brazil; stateProvince: Piauí; municipality: Piracuruca; locality: Parque Nacional de Sete Cidades, Olha d'água dos Milagres; maximumElevationInMeters: 180; verbatimCoordinates: $4^{\circ} 5^{\prime} 31.8^{\prime \prime} \mathrm{S}, 41^{\circ} 40^{\prime} 48.2^{\prime \prime} \mathrm{W}$; samplingProtocol: Pennsylvania light trap; verbatimEventDate: 9.ii.13; individualCount: 3; sex: male; lifeStage: adult; recordedBy: Santos, A.P.M. | Takiya, D.M.; identifiedBy: Inês Corrêa Gonçalves; institutionCode: DZRJ; basisOfRecord: PreservedSpecimen

b. $\quad$ country: Brazil; stateProvince: Piaú́; municipality: Piracuruca; locality: Parque Nacional de Sete Cidades, Cachoeira do Riachão; maximumElevationInMeters: 171; verbatimCoordinates: $4^{\circ} 6^{\prime} 28$ "S, $41^{\circ} 40^{\prime} 13^{\prime \prime} \mathrm{W}$; samplingProtocol: White sheet light trap; verbatimEventDate: 9.ii.13; individualCount: 1; sex: female; lifeStage: immature; recordedBy: Santos, A.P.M.; identifiedBy: Inês Corrêa Gonçalves; institutionCode: DZRJ; basisOfRecord: PreservedSpecimen

Distribution: Brazil: PA, AM, PI!

Notes: New species record for Northeastern Brazil. 


\section{Family Leptophlebiidae}

Notes: New family record for PI.

\section{Genus Farrodes Peters, 1971}

Notes: New genus record for PI.

\section{Farrodes tepui* Domínguez, Molineri \& Peters, 1996}

\section{Materials}

a. country: Brazil; stateProvince: Piauí; municipality: Piracuruca; locality: Parque Nacional de Sete Cidades, Cachoeira do Riachão; maximumElevationInMeters: 171; verbatimCoordinates: $4^{\circ} 6^{\prime} 28$ "S, $41^{\circ} 40^{\prime} 13$ "W; samplingProtocol: Pennsylvania light trap; verbatimEventDate: 19.iv.12; individualCount: 3; sex: male; lifeStage: adult; recordedBy: Takiya, D.M.; identifiedBy: Inês Corrêa Gonçalves; institutionCode: DZRJ; basisOfRecord: PreservedSpecimen

b. $\quad$ country: Brazil; stateProvince: Piauí; municipality: Piracuruca; locality: Parque Nacional de Sete Cidades, Cachoeira do Riachão; maximumElevationInMeters: 171; verbatimCoordinates: $4^{\circ} 6^{\prime} 28^{\prime \prime S}, 41^{\circ} 40^{\prime} 13^{\prime \prime W}$; samplingProtocol: Pennsylvania light trap; verbatimEventDate: 19.iv.12; individualCount: 8; sex: female; lifeStage: adult; recordedBy: Takiya, D.M.; identifiedBy: Inês Corrêa Gonçalves; institutionCode: DZRJ; basisOfRecord: PreservedSpecimen

c. country: Brazil; stateProvince: Piauí; municipality: Piracuruca; locality: Parque Nacional de Sete Cidades, Riacho da Piedade; maximumElevationInMeters: 169; verbatimCoordinates: $4^{\circ} 6^{\prime} 34$ "S, $41^{\circ} 43^{\prime} 39 " \mathrm{~W}$; samplingProtocol: Malaise intercept trap; verbatimEventDate: 21.iv.12; individualCount: 2; sex: male; lifeStage: adult; recordedBy: Rafael, J.A. | Limeira-de-Oliveira, F. | Takiya, D.M. | et al.; identifiedBy: Inês Corrêa Gonçalves; institutionCode: DZRJ; basisOfRecord: PreservedSpecimen

Distribution: Venezuela. Brazil: PI!, CE!, PE.

Notes: New species record for PI.

\section{Genus Fittkaulus Savage \& Peters, 1978}

Notes: New genus record for PI.

\section{Fittkaulus cururuensis Savage, 1986}

\section{Materials}

a. country: Brazil; stateProvince: Piauí; municipality: Piracuruca; locality: Parque Nacional de Sete Cidades, Riacho da Piedade; maximumElevationInMeters: 169;

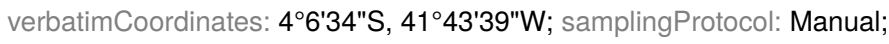
verbatimEventDate: 18.iv.12; individualCount: 1; sex: male; lifeStage: adult; recordedBy: Takiya, D.M.; identifiedBy: Inês Corrêa Gonçalves; institutionCode: DZRJ; basisOfRecord: PreservedSpecimen 
b. $\quad$ country: Brazil; stateProvince: Piauí; municipality: Piracuruca; locality: Parque Nacional de Sete Cidades, Riacho da Piedade; maximumElevationInMeters: 169; verbatimCoordinates: $4^{\circ} 6^{\prime} 34$ "S, $41^{\circ} 43^{\prime} 39$ "W; samplingProtocol: Pennsylvania light trap; verbatimEventDate: 19.iv.12; individualCount: 1; sex: male; lifeStage: adult; recordedBy: Takiya, D.M.; identifiedBy: Inês Corrêa Gonçalves; institutionCode: DZRJ; basisOfRecord: PreservedSpecimen

c. country: Brazil; stateProvince: Piauí; municipality: Piracuruca; locality: Parque Nacional de Sete Cidades, Riacho da Piedade; maximumElevationInMeters: 169; verbatimCoordinates: $4^{\circ} 6^{\prime} 34^{\prime \prime S}, 41^{\circ} 43^{\prime} 39 " \mathrm{~W}$; samplingProtocol: Pennsylvania light trap; verbatimEventDate: 19.iv.12; individualCount: 1; sex: female; lifeStage: adult; recordedBy: Takiya, D.M.; identifiedBy: Inês Corrêa Gonçalves; institutionCode: DZRJ; basisOfRecord: PreservedSpecimen

d. $\quad$ country: Brazil; stateProvince: Piauí; municipality: Piracuruca; locality: Parque Nacional de Sete Cidades, Cachoeira do Riachão; maximumElevationInMeters: 171; verbatimCoordinates: $4^{\circ} 6^{\prime} 28$ "S, $41^{\circ} 40^{\prime} 13$ "W; samplingProtocol: Pennsylvania light trap; verbatimEventDate: 20.iv.12; individualCount: 1; sex: male; lifeStage: adult; recordedBy: Takiya, D.M. | Rafael, J.A.; identifiedBy: Inês Corrêa Gonçalves; institutionCode: DZRJ; basisOfRecord: PreservedSpecimen

e. $\quad$ country: Brazil; stateProvince: Piauí; municipality: Piracuruca; locality: Parque Nacional de Sete Cidades, Riacho da Piedade; maximumElevationInMeters: 169; verbatimCoordinates: $4^{\circ} 6^{\prime} 34$ "S, $41^{\circ} 43^{\prime} 39$ "W; samplingProtocol: Malaise intercept trap; verbatimEventDate: 21.iv.12; individualCount: 3; sex: female; lifeStage: adult; recordedBy: Rafael, J.A. | Limeira-de-Oliveira, F. | Takiya, D.M. | et al.; identifiedBy: Inês Corrêa Gonçalves; institutionCode: DZRJ; basisOfRecord: PreservedSpecimen

f. country: Brazil; stateProvince: Piauí; municipality: Piracuruca; locality: Parque Nacional de Sete Cidades, Riacho da Piedade; maximumElevationInMeters: 169; verbatimCoordinates: $4^{\circ} 6^{\prime} 34$ "S, $41^{\circ} 43^{\prime} 39$ "W; sampling Protocol: Malaise intercept trap; verbatimEventDate: 21.iv.12; individualCount: 2; sex: male; lifeStage: adult; recordedBy: Rafael, J.A. | Limeira-de-Oliveira, F. | Takiya, D.M. | et al.; identifiedBy: Inês Corrêa Gonçalves; institutionCode: DZRJ; basisOfRecord: PreservedSpecimen

g. country: Brazil; stateProvince: Piauí; municipality: Piracuruca; locality: Parque Nacional de Sete Cidades, Cachoeira do Riachão; maximumElevationInMeters: 171; verbatimCoordinates: $4^{\circ} 6^{\prime 2} 28^{\prime \prime S}, 41^{\circ} 40^{\prime} 13$ "W; samplingProtocol: Pennsylvania light trap; verbatimEventDate: 20.iv.12; individualCount: 5; sex: female; lifeStage: adult; recordedBy: Takiya, D.M. | Rafael, J.A.; identifiedBy: Inês Corrêa Gonçalves; institutionCode: DZRJ; basisOfRecord: PreservedSpecimen

Distribution: Brazil: PA, PI!, PE, MT, ES.

Notes: New species record for PI.

\section{Genus Miroculis Edmunds, 1963}

Notes: New genus record for PI. 


\section{Miroculis sp. 1}

\section{Materials}

a. country: Brazil; stateProvince: Piauí; municipality: Piracuruca; locality: Parque Nacional de Sete Cidades, Cachoeira do Riachão; maximumElevationInMeters: 171; verbatimCoordinates: $4^{\circ} 6^{\prime} 28$ "S, $41^{\circ} 40^{\prime} 13^{\prime \prime W}$; samplingProtocol: Pennsylvania light trap; verbatimEventDate: 19.iv.12; individualCount: 2; sex: male; lifeStage: adult; recordedBy: Takiya, D.M.; identifiedBy: Inês Corrêa Gonçalves; institutionCode: DZRJ; basisOfRecord: PreservedSpecimen

b. country: Brazil; stateProvince: Piauí; municipality: Piracuruca; locality: Parque Nacional de Sete Cidades, Cachoeira do Riachão; maximumElevationInMeters: 171; verbatimCoordinates: $4^{\circ} 6^{\prime} 28^{\prime \prime S}, 41^{\circ} 40^{\prime} 13$ "W; samplingProtocol: Pennsylvania light trap; verbatimEventDate: 20.iv.12; individualCount: 1; sex: male; lifeStage: adult; recordedBy: Takiya, D.M. | Rafael, J.A.; identifiedBy: Inês Corrêa Gonçalves; institutionCode: DZRJ; basisOfRecord: PreservedSpecimen

c. country: Brazil; stateProvince: Piauí; municipality: Piracuruca; locality: Parque Nacional de Sete Cidades, Riacho da Piedade; maximumElevationInMeters: 169; verbatimCoordinates: $4^{\circ} 6^{\prime} 34$ "S, 41 ${ }^{\circ} 43^{\prime} 39$ "W; samplingProtocol: Malaise intercept trap; verbatimEventDate: 21.iv.12; individualCount: 3; sex: female; lifeStage: adult; recordedBy: Rafael, J.A. | Limeira-de-Oliveira, F. | Takiya, D.M. | et al.; identifiedBy: Inês Corrêa Gonçalves; institutionCode: DZRJ; basisOfRecord: PreservedSpecimen

Notes: Undescribed species.

\section{Genus Simothraulopsis Demoulin, 1966}

Notes: New genus record for PI.

\section{Simothraulopsis demerara (Traver, 1947)}

\section{Materials}

a. country: Brazil; stateProvince: Piauí; municipality: Piracuruca; locality: Parque Nacional de Sete Cidades, Cachoeira do Riachão; maximumElevationInMeters: 171; verbatimCoordinates: $4^{\circ} 6^{\prime} 28$ "S, $41^{\circ} 40^{\prime} 13^{\prime \prime} \mathrm{W}$; samplingProtocol: Pennsylvania light trap; verbatimEventDate: 18.iv.12; individualCount: 3; sex: male; lifeStage: adult; recordedBy: Takiya, D.M.; identifiedBy: Inês Corrêa Gonçalves; institutionCode: DZRJ; basisOfRecord: PreservedSpecimen

b. country: Brazil; stateProvince: Piauí; municipality: Piracuruca; locality: Parque Nacional de Sete Cidades, Cachoeira do Riachão; maximumElevationInMeters: 171; verbatimCoordinates: $4^{\circ} 6^{\prime} 28^{\prime \prime S}, 41^{\circ} 40^{\prime} 13 " \mathrm{~W}$; samplingProtocol: Pennsylvania light trap; verbatimEventDate: 18.iv.12; individualCount: 2; sex: female; lifeStage: adult; recordedBy: Takiya, D.M.; identifiedBy: Inês Corrêa Gonçalves; institutionCode: DZRJ; basisOfRecord: PreservedSpecimen

c. country: Brazil; stateProvince: Piauí; municipality: Piracuruca; locality: Parque Nacional de Sete Cidades, Cachoeira do Riachão; maximumElevationInMeters: 171; verbatimCoordinates: $4^{\circ} 6^{\prime} 28^{\prime \prime S}, 41^{\circ} 40^{\prime} 13^{\prime \prime W}$; samplingProtocol: Pennsylvania light trap; verbatimEventDate: 19.iv.12; individualCount: 1; sex: male; lifeStage: adult; recordedBy: 
Takiya, D.M.; identifiedBy: Inês Corrêa Gonçalves; institutionCode: DZRJ; basisOfRecord: PreservedSpecimen

d. country: Brazil; stateProvince: Piauí; municipality: Piracuruca; locality: Parque Nacional de Sete Cidades, Cachoeira do Riachão; maximumElevationInMeters: 171; verbatimCoordinates: $4^{\circ} 6^{\prime} 28^{\prime \prime S}, 41^{\circ} 40^{\prime} 13^{\prime \prime W}$; samplingProtocol: Pennsylvania light trap; verbatimEventDate: 19.iv.12; individualCount: 2; sex: female; lifeStage: adult; recordedBy: Takiya, D.M.; identifiedBy: Inês Corrêa Gonçalves; institutionCode: DZRJ; basisOfRecord: PreservedSpecimen

e. country: Brazil; stateProvince: Piauí; municipality: Piracuruca; locality: Parque Nacional de Sete Cidades, Riacho da Piedade; maximumElevationInMeters: 169; verbatimCoordinates: $4^{\circ} 6{ }^{\prime} 34^{\prime \prime S}, 41^{\circ} 43^{\prime} 39$ "W; samplingProtocol: Pennsylvania light trap; verbatimEventDate: 19.iv.12; individualCount: 1; sex: female; lifeStage: adult; recordedBy: Takiya, D.M.; identifiedBy: Inês Corrêa Gonçalves; institutionCode: DZRJ; basisOfRecord: PreservedSpecimen

f. country: Brazil; stateProvince: Piauí; municipality: Piracuruca; locality: Parque Nacional de Sete Cidades, Cachoeira do Riachão; maximumElevationInMeters: 171; verbatimCoordinates: $4^{\circ} 6^{\prime} 28^{\prime \prime S}, 41^{\circ} 40^{\prime} 13$ "W; samplingProtocol: Pennsylvania light trap; verbatimEventDate: 20.iv.12; individualCount: 3; sex: male; lifeStage: adult; recordedBy: Takiya, D.M. | Rafael, J.A.; identifiedBy: Inês Corrêa Gonçalves; institutionCode: DZRJ; basisOfRecord: PreservedSpecimen

g. country: Brazil; stateProvince: Piauí; municipality: Piracuruca; locality: Parque Nacional de Sete Cidades, Riacho da Piedade; maximumElevationInMeters: 169; verbatimCoordinates: $4^{\circ} 6^{\prime} 34$ "S, 4143'39"W; samplingProtocol: Pennsylvania light trap; verbatimEventDate: 22.iv.12; individualCount: 1; sex: male; lifeStage: adult; recordedBy: Takiya, D.M.; identifiedBy: Inês Corrêa Gonçalves; institutionCode: DZRJ; basisOfRecord: PreservedSpecimen

h. country: Brazil; stateProvince: Piauí; municipality: Piracuruca; locality: Parque Nacional de Sete Cidades, Olha d'água dos Milagres; maximumElevationInMeters: 180; verbatimCoordinates: $4^{\circ} 5^{\prime} 31.8^{\prime \prime} \mathrm{S}, 4^{\circ} 40^{\prime} 48.2^{\prime \prime} \mathrm{W}$; samplingProtocol: White sheet light trap; verbatimEventDate: 9.ii.13; individualCount: 1; sex: male; lifeStage: adult; recordedBy:

Santos, A.P.M. | Takiya, D.M.; identifiedBy: Inês Corrêa Gonçalves; institutionCode: DZRJ; basisOfRecord: PreservedSpecimen

i. country: Brazil; stateProvince: Piauí; municipality: Piracuruca; locality: Parque Nacional de Sete Cidades, Poço do Bananeira; maximumElevationInMeters: 158; verbatimCoordinates: $4^{\circ} 5^{\prime} 55.8^{\prime \prime} \mathrm{S}, 4^{\circ} 40^{\prime} 33.8^{\prime \prime} \mathrm{W}$; samplingProtocol: Pennsylvania light trap; verbatimEventDate: 10.ii.13; individualCount: 1; sex: female; lifeStage: adult; recordedBy: Santos, A.P.M. | Takiya, D.M.; identifiedBy: Inês Corrêa Gonçalves; institutionCode: DZRJ; basisOfRecord: PreservedSpecimen

j. country: Brazil; stateProvince: Piauí; municipality: Piracuruca; locality: Parque Nacional de Sete Cidades, Poço do Bananeira; maximumElevationInMeters: 158; verbatimCoordinates: $4^{\circ} 5^{\prime} 55.8^{\prime \prime} \mathrm{S}, 41^{\circ} 40^{\prime} 33.8^{\prime \prime} \mathrm{W}$; samplingProtocol: Pennsylvania light trap; verbatimEventDate: 11.ii.13; individualCount: 6; sex: female; lifeStage: adult; recordedBy: Santos, A.P.M. | Takiya, D.M.; identifiedBy: Inês Corrêa Gonçalves; institutionCode: DZRJ; basisOfRecord: PreservedSpecimen

Distribution: Colombia. Venezuela. Guyana. Suriname. French Guiana. Brazil: PA, AM, PI!, PE, ES. Ecuador. Bolivia. Argentina.

Notes: New species record for PI. 


\section{Genus Thraulodes Ulmer, 1920}

Notes: New genus record for PI.

\section{Thraulodes sp. 1}

\section{Material}

a. country: Brazil; stateProvince: Piauí; municipality: Piracuruca; locality: Parque Nacional de Sete Cidades, Riacho da Bananeira; maximumElevationInMeters: 189; verbatimCoordinates: $4^{\circ} 5^{\prime} 59^{\prime \prime S}, 41^{\circ} 40^{\prime} 48 " \mathrm{~W}$; samplingProtocol: Malaise intercept trap; verbatimEventDate: 19.iv.12; individualCount: 1; sex: female; lifeStage: adult; recordedBy: Rafael, J.A. | Limeira-de-Oliveira, F. | Takiya, D.M. | et al.; identifiedBy: Inês Corrêa Gonçalves; institutionCode: DZRJ; basisOfRecord: PreservedSpecimen

\section{Genus Ulmeritoides Traver, 1959}

Notes: New genus record for PI.

\section{Ulmeritoides flavopedes (Spieth, 1943)}

\section{Materials}

a. country: Brazil; stateProvince: Piauí; municipality: Piracuruca; locality: Parque Nacional de Sete Cidades, Poço do Bananeira; maximumElevationInMeters: 158; verbatimCoordinates: $4^{\circ} 5^{\prime} 55.8^{\prime \prime} \mathrm{S}, 4^{\circ} 40^{\prime} 33.8^{\prime \prime} \mathrm{W}$; sampling Protocol: Pennsylvania light trap; verbatimEventDate: 10.ii.13; individualCount: 1; sex: female; lifeStage: adult; recordedBy: Santos, A.P.M. | Takiya, D.M.; identifiedBy: Inês Corrêa Gonçalves; institutionCode: DZRJ; basisOfRecord: PreservedSpecimen

b. $\quad$ country: Brazil; stateProvince: Piauí; municipality: Piracuruca; locality: Parque Nacional de Sete Cidades, Poço do Bananeira; maximumElevationInMeters: 158; verbatimCoordinates: $4^{\circ} 5^{\prime} 55.8^{\prime \prime} \mathrm{S}, 4^{\circ} 40^{\prime} 33.8^{\prime \prime} \mathrm{W}$; sampling Protocol: Pennsylvania light trap; verbatimEventDate: 11.ii.13; individualCount: 1; sex: male; lifeStage: adult; recordedBy: Santos, A.P.M. | Takiya, D.M.; identifiedBy: Inês Corrêa Gonçalves; institutionCode: DZRJ; basisOfRecord: PreservedSpecimen

c. country: Brazil; stateProvince: Piauí; municipality: Piracuruca; locality: Parque Nacional de Sete Cidades, Poço do Bananeira; maximumElevationInMeters: 158; verbatimCoordinates: $4^{\circ} 5^{\prime} 55.8^{\prime \prime} \mathrm{S}, 4^{\circ} 40^{\prime} 33.8^{\prime \prime} \mathrm{W}$; sampling Protocol: Pennsylvania light trap; verbatimEventDate: 11.ii.13; individualCount: 1; sex: female; lifeStage: adult; recordedBy: Santos, A.P.M. | Takiya, D.M.; identifiedBy: Inês Corrêa Gonçalves; institutionCode: DZRJ; basisOfRecord: PreservedSpecimen

d. country: Brazil; stateProvince: Piauí; municipality: Piracuruca; locality: Parque Nacional de Sete Cidades, Poço do Bananeira; maximumElevationInMeters: 158; verbatimCoordinates: $4^{\circ} 5^{\prime} 55.8^{\prime \prime} \mathrm{S}, 41^{\circ} 40^{\prime} 33.8^{\prime \prime} \mathrm{W}$; sampling Protocol: Pennsylvania light trap; verbatimEventDate: 9.ii.13; individualCount: 1; sex: male; lifeStage: adult; recordedBy: Santos, A.P.M. | Takiya, D.M.; identifiedBy: Inês Corrêa Gonçalves; institutionCode: DZRJ; basisOfRecord: PreservedSpecimen

e. country: Brazil; stateProvince: Piauí; municipality: Piracuruca; locality: Parque Nacional de Sete Cidades, Cachoeira do Riachão; maximumElevationInMeters: 171; verbatimCoordinates: $4^{\circ} 6^{\prime} 28^{\prime \prime S}, 41^{\circ} 40^{\prime} 13^{\prime \prime W}$; samplingProtocol: Pennsylvania light trap; 
verbatimEventDate: 19.iv.12; individualCount: 7; sex: male; lifeStage: adult; recordedBy: Takiya, D.M.; identifiedBy: Inês Corrêa Gonçalves; institutionCode: DZRJ; basisOfRecord: PreservedSpecimen

f. country: Brazil; stateProvince: Piauí; municipality: Piracuruca; locality: Parque Nacional de Sete Cidades, Cachoeira do Riachão; maximumElevationInMeters: 171; verbatimCoordinates: $4^{\circ} 6^{\prime} 28^{\prime \prime S}, 41^{\circ} 40^{\prime} 13^{\prime \prime W}$; samplingProtocol: Pennsylvania light trap; verbatimEventDate: 19.iv.12; individualCount: 3; sex: female; lifeStage: adult; recordedBy: Takiya, D.M.; identifiedBy: Inês Corrêa Gonçalves; institutionCode: DZRJ; basisOfRecord: PreservedSpecimen

g. country: Brazil; stateProvince: Piauí; municipality: Piracuruca; locality: Parque Nacional de Sete Cidades, Cachoeira do Riachão; maximumElevationInMeters: 171; verbatimCoordinates: $4^{\circ} 6^{\prime} 28$ "S, $41^{\circ} 40^{\prime} 13$ "W; samplingProtocol: Pennsylvania light trap; verbatimEventDate: 20.iv.12; individualCount: 14; sex: male; lifeStage: adult; recordedBy: Takiya, D.M. | Rafael, J.A.; identifiedBy: Inês Corrêa Gonçalves; institutionCode: DZRJ; basisOfRecord: PreservedSpecimen

h. country: Brazil; stateProvince: Piauí; municipality: Piracuruca; locality: Parque Nacional de Sete Cidades, Cachoeira do Riachão; maximumElevationInMeters: 171; verbatimCoordinates: $4^{\circ} 6^{\prime} 28^{\prime \prime} \mathrm{S}, 41^{\circ} 40^{\prime} 13^{\prime \prime} \mathrm{W}$; samplingProtocol: Pennsylvania light trap; verbatimEventDate: 20.iv.12; individualCount: 2; sex: female; lifeStage: adult; recordedBy: Takiya, D.M. | Rafael, J.A.; identifiedBy: Inês Corrêa Gonçalves; institutionCode: DZRJ; basisOfRecord: PreservedSpecimen

Distribution: Suriname. Brazil: RR, PI!, PE, MT.

Notes: New species record for PI.

\section{Family Polymitarcyidae}

\section{Genus Campsurus Eaton, 1868}

\section{Campsurus truncatus Ulmer, 1920}

\section{Materials}

a. country: Brazil; stateProvince: Piauí; municipality: Piracuruca; locality: Parque Nacional de Sete Cidades, Cachoeira do Riachão; maximumElevationInMeters: 171; verbatimCoordinates: $4^{\circ} 6^{\prime} 28$ "S, $41^{\circ} 40^{\prime} 13^{\prime \prime} \mathrm{W}$; samplingProtocol: Pennsylvania light trap; verbatimEventDate: 18.iv.12; individualCount: 1; sex: female; lifeStage: adult; recordedBy: Takiya, D.M.; identifiedBy: Inês Corrêa Gonçalves; institutionCode: DZRJ; basisOfRecord: PreservedSpecimen

b. $\quad$ country: Brazil; stateProvince: Piauí; municipality: Piracuruca; locality: Parque Nacional de Sete Cidades, Cachoeira do Riachão; maximumElevationInMeters: 171; verbatimCoordinates: $4^{\circ} 6^{\prime} 28$ "S, $41^{\circ} 40^{\prime} 13 " \mathrm{~W}$; samplingProtocol: Pennsylvania light trap; verbatimEventDate: 18.iv.12; individualCount: 1; sex: male; lifeStage: adult; recordedBy: Takiya, D.M.; identifiedBy: Inês Corrêa Gonçalves; institutionCode: DZRJ; basisOfRecord: PreservedSpecimen

c. country: Brazil; stateProvince: Piauí; municipality: Piracuruca; locality: Parque Nacional de Sete Cidades, Cachoeira do Riachão; maximumElevationInMeters: 171; verbatimCoordinates: $4^{\circ} 6^{\prime} 28^{\prime \prime} \mathrm{S}, 41^{\circ} 40^{\prime} 13^{\prime \prime} \mathrm{W}$; samplingProtocol: Pennsylvania light trap; 
verbatimEventDate: 19.iv.12; individualCount: 2; sex: female; lifeStage: adult; recordedBy: Takiya, D.M.; identifiedBy: Inês Corrêa Gonçalves; institutionCode: DZRJ; basisOfRecord: PreservedSpecimen

d. country: Brazil; stateProvince: Piauí; municipality: Piracuruca; locality: Parque Nacional de Sete Cidades, Olho d'água Piscina do Bacuri; maximumElevationlnMeters: 171; verbatimCoordinates: $4^{\circ} 6^{\prime} 1.2^{\prime \prime} \mathrm{S}, 41^{\circ} 42^{\prime} 38.8^{\prime \prime} \mathrm{W}$; sampling Protocol: White sheet light trap; verbatimEventDate: 20.iv.12; individualCount: 5; sex: female; lifeStage: adult; recordedBy: Takiya, D.M.; identifiedBy: Inês Corrêa Gonçalves; institutionCode: DZRJ; basisOfRecord: PreservedSpecimen

e. $\quad$ country: Brazil; stateProvince: Piauí; municipality: Piracuruca; locality: Parque Nacional de Sete Cidades, Cachoeira do Riachão; maximumElevationInMeters: 171; verbatimCoordinates: $4^{\circ} 6^{\prime} 28$ "S, $41^{\circ} 40^{\prime} 13$ "W; samplingProtocol: Pennsylvania light trap; verbatimEventDate: 12.ii.13; individualCount: 2; sex: female; lifeStage: adult; recordedBy: Santos, A.P.M. | Takiya, D.M.; identifiedBy: Inês Corrêa Gonçalves; institutionCode: DZRJ; basisOfRecord: PreservedSpecimen

f. country: Brazil; stateProvince: Piauí; municipality: Piracuruca; locality: Parque Nacional de Sete Cidades, Alojamento; maximumElevationInMeters: 193; verbatimCoordinates: $4^{\circ}$ 5'57"S, 4142'34"W; samplingProtocol: White sheet light trap; verbatimEventDate: 8.ii.13; individualCount: 1; sex: female; lifeStage: adult; recordedBy: Santos, A.P.M. | Takiya, D.M.; identifiedBy: Inês Corrêa Gonçalves; institutionCode: DZRJ; basisOfRecord: PreservedSpecimen

g. country: Brazil; stateProvince: Piauí; municipality: Piracuruca; locality: Parque Nacional de Sete Cidades, Cachoeira do Riachão; maximumElevationInMeters: 171; verbatimCoordinates: $4^{\circ} 6^{\prime} 28^{\prime \prime S}, 41^{\circ} 40^{\prime} 13 " \mathrm{~W}$; sampling Protocol: White sheet light trap; verbatimEventDate: 8.ii.13; individualCount: 3; sex: female; lifeStage: adult; recordedBy: Santos, A.P.M. | Takiya, D.M.; identifiedBy: Inês Corrêa Gonçalves; institutionCode: DZRJ; basisOfRecord: PreservedSpecimen

h. country: Brazil; stateProvince: Piauí; municipality: Piracuruca; locality: Parque Nacional de Sete Cidades, Cachoeira do Riachão; maximumElevationInMeters: 171; verbatimCoordinates: $4^{\circ} 6^{\prime} 28^{\prime \prime S}, 41^{\circ} 40^{\prime} 13^{\prime \prime} \mathrm{W}$; sampling Protocol: White sheet light trap; verbatimEventDate: 8.ii.13; individualCount: 2; sex: female; lifeStage: adult; recordedBy: Santos, A.P.M. | Takiya, D.M.; identifiedBy: Inês Corrêa Gonçalves; institutionCode: DZRJ; basisOfRecord: PreservedSpecimen

i. country: Brazil; stateProvince: Piauí; municipality: Piracuruca; locality: Parque Nacional de Sete Cidades, Olho d'água Piscina do Bacuri; maximumElevationlnMeters: 171; verbatimCoordinates: $4^{\circ} 6^{\prime} 1.2^{\prime \prime} \mathrm{S}, 41^{\circ} 42^{\prime} 38.8^{\prime \prime} \mathrm{W}$; samplingProtocol: White sheet light trap; verbatimEventDate: 8.ii.13; individualCount: 1; sex: female; lifeStage: adult; recordedBy: Santos, A.P.M. | Takiya, D.M.; identifiedBy: Inês Corrêa Gonçalves; institutionCode: DZRJ; basisOfRecord: PreservedSpecimen

j. country: Brazil; stateProvince: Piauí; municipality: Piracuruca; locality: Parque Nacional de Sete Cidades, Poço do Bananeira; maximumElevationInMeters: 158; verbatimCoordinates: $4^{\circ} 5^{\prime} 55.8^{\prime \prime S}, 41^{\circ} 40^{\prime} 33.8^{\prime \prime} \mathrm{W}$; samplingProtocol: Pennsylvania light trap; verbatimEventDate: 9.ii.13; individualCount: 2; sex: female; lifeStage: adult; recordedBy: Santos, A.P.M. | Takiya, D.M.; identifiedBy: Inês Corrêa Gonçalves; institutionCode: DZRJ; basisOfRecord: PreservedSpecimen

Distribution: Colombia. Brazil: PI!, PE, ES, RJ. Peru. Bolivia.

Notes: New species record for PI. 


\section{Order Hemiptera}

\section{Infraorder Gerromorpha}

\section{Family Gerridae}

\section{Genus Neogerris Matsumura, 1913}

Notes: Genus firstly recorded from PI in Cordeiro and Moreira 2015.

\section{Neogerris lubricus (White, 1879)}

\section{Material}

a. country: Brazil; stateProvince: Piauí; municipality: Piracuruca; locality: Parque Nacional de Sete Cidades, Cachoeira do Riachão; maximumElevationInMeters: 171; verbatimCoordinates: $4^{\circ} 6^{\prime} 28$ "S, $41^{\circ} 40^{\prime} 13^{\prime \prime} \mathrm{W}$; samplingProtocol: Manual; verbatimEventDate: 12.ii.13; individualCount: 1; sex: male; lifeStage: adult; recordedBy: Santos, A.P.M. | Takiya, D.M.; identifiedBy: Isabelle da R. S. Cordeiro; institutionCode: DZRJ; basisOfRecord: PreservedSpecimen

Distribution: Panama. Trinidad \& Tobago. Colombia. Guyana. Suriname. Brazil: AP, PA, AM, PI, MT, RO, BA, MG, MS, SP, RJ. Ecuador. Peru. Bolivia. Paraguay. Argentina.

Notes: Species firstly recorded from PI in Cordeiro and Moreira 2015.

\section{Family Mesoveliidae}

Notes: New family record for PI.

\section{Genus Mesovelia Mulsant \& Rey, 1852}

Notes: New genus record for PI.

\section{Mesovelia sp. 1}

\section{Material}

a. country: Brazil; stateProvince: Piauí; municipality: Piracuruca; locality: Parque Nacional de Sete Cidades, Cachoeira do Riachão; maximumElevationInMeters: 171; verbatimCoordinates: $4^{\circ} 6^{\prime} 28^{\prime \prime} \mathrm{S}, 41^{\circ} 40^{\prime} 13^{\prime \prime} \mathrm{W}$; samplingProtocol: Manual; verbatimEventDate: 9.ii.13; individualCount: 1; lifeStage: immature; recordedBy: Santos, A.P.M. | Takiya, D.M.; identifiedBy: Isabelle da R. S. Cordeiro; institutionCode: DZRJ; basisOfRecord: PreservedSpecimen 


\section{Family Veliidae}

Notes: Family firstly recorded from PI in Cordeiro and Moreira 2015.

\section{Genus Microvelia Westwood, 1834}

Notes: Genus firstly recorded from PI in Cordeiro and Moreira 2015.

\section{Microvelia ayacuchana Drake \& Maldonado Capriles 1952}

\section{Materials}

a. $\quad$ country: Brazil; stateProvince: Piauí; municipality: Piracuruca; locality: Parque Nacional de Sete Cidades, Cachoeira do Riachão; maximumElevationInMeters: 171; verbatimCoordinates: $4^{\circ} 6^{\prime} 28 " \mathrm{~S}, 41^{\circ} 40^{\prime} 13 " \mathrm{~W}$; samplingProtocol: Manual; verbatimEventDate: 9.ii.13; individualCount: 1; sex: male; lifeStage: adult; recordedBy: Santos, A.P.M. | Takiya, D.M.; identifiedBy: Isabelle da R. S. Cordeiro; institutionCode: DZRJ; basisOfRecord: PreservedSpecimen

b. country: Brazil; stateProvince: Piauí; municipality: Piracuruca; locality: Parque Nacional de Sete Cidades, Cachoeira do Riachão; maximumElevationInMeters: 171; verbatimCoordinates: 46'28"S, 4140'13"W; samplingProtocol: Manual; verbatimEventDate: 9.ii.13; individualCount: 1; sex: female; lifeStage: adult; recordedBy: Santos, A.P.M. | Takiya, D.M.; identifiedBy: Isabelle da R. S. Cordeiro; institutionCode: DZRJ; basisOfRecord: PreservedSpecimen

Distribution: Venezuela. Guyana. Suriname. Brazil: PA, PI, ES.

Notes: Species firstly recorded from Northeastern Brazil in Cordeiro and Moreira 2015.

\section{Microvelia pulchella Westwood, 1834}

\section{Materials}

a. $\quad$ country: Brazil; stateProvince: Piauí; municipality: Piracuruca; locality: Parque Nacional de Sete Cidades, Rio da Bananeira; maximumElevationInMeters: 189; verbatimCoordinates: 45'59"S, 4140'48"W; samplingProtocol: Manual; verbatimEventDate: 8.ii.13; individualCount: 5; sex: male; lifeStage: adult; recordedBy: Santos, A.P.M. | Takiya, D.M.; identifiedBy: Isabelle da R. S. Cordeiro; institutionCode: DZRJ; basisOfRecord: PreservedSpecimen

b. country: Brazil; stateProvince: Piauí; municipality: Piracuruca; locality: Parque Nacional de Sete Cidades, Rio da Bananeira; maximumElevationInMeters: 189; verbatimCoordinates: $4^{\circ} 5^{\prime} 59$ "S, 4140'48"W; samplingProtocol: Manual; verbatimEventDate: 8.ii.13; individualCount: 2; sex: female; lifeStage: adult; recordedBy: Santos, A.P.M. | Takiya, D.M.; identifiedBy: Isabelle da R. S. Cordeiro; institutionCode: DZRJ; basisOfRecord: PreservedSpecimen

Distribution: Canada. USA. Mexico. Guatemala. Costa Rica. Panama. Bahamas. Cuba. Dominican Republic. Cayman Islands. Jamaica. Puerto Rico. U.S. Virgin Islands. Anguilla. St. Martin. Saba. St. Kitts \& Nevis. Guadeloupe. Martinique. Aruba. St. 
Vincent \& Grenadines. Barbados. Curaçao. Klein Curaçao. Bonaire. Klein Bonaire. Grenada. Trinidad and Tobago. Colombia. Venezuela. Brazil: PA, AM, MA, PI, PE, BA, AL, MG, MS, ES, SP, RJ, SC. Ecuador. Peru. Argentina.

Notes: Species firstly recorded from PI in Cordeiro and Moreira 2015.

\section{Genus Platyvelia Polhemus \& Polhemus, 1993}

Notes: Genus firstly recorded from PI in Cordeiro and Moreira 2015.

\section{Platyvelia brachialis (Stål, 1860)}

\section{Material}

a. country: Brazil; stateProvince: Piauí; municipality: Piracuruca; locality: Parque Nacional de Sete Cidades, Olha d'água dos Milagres; maximumElevationInMeters: 180; verbatimCoordinates: $4^{\circ} 5^{\prime} 31.8^{\prime \prime} \mathrm{S}, 41^{\circ} 40^{\prime} 48.2^{\prime \prime} \mathrm{W}$; samplingProtocol: Manual; verbatimEventDate: 11.ii.13; individualCount: 1; sex: female; lifeStage: adult; recordedBy: Santos, A.P.M. | Takiya, D.M.; identifiedBy: Isabelle da R. S. Cordeiro; institutionCode: DZRJ; basisOfRecord: PreservedSpecimen

Distribution: USA. Mexico. Guatemala. Nicaragua. Costa Rica. Panama. Cuba. Dominican Republic. Jamaica. Grenada. Trinidad and Tobago. Suriname. Brazil: PI, PE, MT, GO, MG, MS, ES, RJ, SC. Peru. Argentina.

Notes: Species firstly recorded from PI in Cordeiro and Moreira 2015.

\section{Infraorder Nepomorpha}

\section{Family Belostomatidae}

\section{Genus Belostoma Latreille, 1807}

\section{Belostoma sp. 1*}

Material

a. country: Brazil; stateProvince: Piauí; municipality: Piracuruca; locality: Parque Nacional de Sete Cidades, Cachoeira do Riachão; maximumElevationInMeters: 171; verbatimCoordinates: $4^{\circ} 6^{\prime} 28^{\prime \prime} \mathrm{S}, 41^{\circ} 40^{\prime} 13^{\prime \prime} \mathrm{W}$; samplingProtocol: Manual; verbatimEventDate: 12.ii.13; individualCount: 1; sex: female; lifeStage: adult; recordedBy: Santos, A.P.M. | Takiya, D.M.; identifiedBy: Julianna Freires Barbosa; institutionCode: DZRJ; basisOfRecord: PreservedSpecimen 


\section{Family Corixidae}

Notes: New family record for PI.

\section{Genus Tenagobia Bergroth, 1899}

Notes: New genus record for PI.

\section{Tenagobia (Incertagobia) sp.}

\section{Material}

a. $\quad$ country: Brazil; stateProvince: Piauí; municipality: Piracuruca; locality: Parque Nacional de Sete Cidades, Cachoeira do Riachão; maximumElevationInMeters: 171; verbatimCoordinates: $4^{\circ} 6^{\prime} 28$ "S, 4140'13"W; samplingProtocol: Manual; verbatimEventDate: 9.ii.13; individualCount: 1; sex: male; lifeStage: adult; recordedBy: Santos, A.P.M. | Takiya, D.M.; identifiedBy: Isabelle da R. S. Cordeiro; institutionCode: DZRJ; basisOfRecord: PreservedSpecimen

\section{Family Gelastocoridae}

Notes: New family record for PI.

\section{Genus Gelastocoris Kirkaldy, 1897}

Notes: New genus record for PI.

\section{Gelastocoris sp. 1}

\section{Materials}

a. country: Brazil; stateProvince: Piauí; municipality: Piracuruca; locality: Parque Nacional de Sete Cidades, Riacho da Bananeira; maximumElevationInMeters: 189; verbatimCoordinates: $4^{\circ} 5^{\prime} 59 " \mathrm{~S}, 41^{\circ} 40^{\prime} 48^{\prime \prime} \mathrm{W}$; samplingProtocol: Manual; verbatimEventDate: 8.ii.13; individualCount: 1; sex: female; lifeStage: adult; recordedBy: Santos, A.P.M. | Takiya, D.M.; identifiedBy: Julianna Freires Barbosa; institutionCode: DZRJ; basisOfRecord: PreservedSpecimen

b. $\quad$ country: Brazil; stateProvince: Piauí; municipality: Piracuruca; locality: Parque Nacional de Sete Cidades, Olha d'água dos Milagres; maximumElevationInMeters: 180; verbatimCoordinates: 45'31.8"S, 4140'48.2"W; samplingProtocol: Manual; verbatimEventDate: 8.ii.13; individualCount: 1; lifeStage: immature; recordedBy: Santos, A.P.M. | Takiya, D.M.; identifiedBy: Isabelle da R. S. Cordeiro; institutionCode: DZRJ; basisOfRecord: PreservedSpecimen 


\section{Family Pleidae}

Notes: New family record for PI.

Genus Neoplea Esaki \& china, 1928

Notes: New genus record for PI.

\section{Neoplea sp. 1}

\section{Materials}

a. country: Brazil; stateProvince: Piauí; municipality: Piracuruca; locality: Parque Nacional de Sete Cidades, Cachoeira do Riachão; maximumElevationInMeters: 171; verbatimCoordinates: $4^{\circ} 6^{\prime} 28$ "S, $41^{\circ} 40^{\prime} 13$ "W; samplingProtocol: Pennsylvania light trap; verbatimEventDate: 19.iv.12; individualCount: 2; sex: male; lifeStage: adult; recordedBy: Takiya, D.M.; identifiedBy: Julianna Freires Barbosa; institutionCode: DZRJ; basisOfRecord: PreservedSpecimen

b. country: Brazil; stateProvince: Piauí; municipality: Piracuruca; locality: Parque Nacional de Sete Cidades, Cachoeira do Riachão; maximumElevationInMeters: 171; verbatimCoordinates: $4^{\circ} 6^{\prime} 28$ "S, $41^{\circ} 40^{\prime} 13$ "W; samplingProtocol: Pennsylvania light trap; verbatimEventDate: 19.iv.12; individualCount: 4; sex: female; lifeStage: adult; recordedBy: Takiya, D.M.; identifiedBy: Julianna Freires Barbosa; institutionCode: DZRJ; basisOfRecord: PreservedSpecimen

\section{Family Naucoridae}

Notes: New family record for PI.

\section{Genus Pelocoris Stal, 1876}

Notes: New genus record for Northeastern Brazil.

\section{Pelocoris sp. 1}

\section{Material}

a. country: Brazil; stateProvince: Piauí; municipality: Piracuruca; locality: Parque Nacional de Sete Cidades, Cachoeira do Riachão; maximumElevationInMeters: 171; verbatimCoordinates: 46'28"S, 4140'13"W; samplingProtocol: Manual; verbatimEventDate: 9.ii.13; individualCount: 1; sex: female; lifeStage: adult; recordedBy: Santos, A.P.M. | Takiya, D.M.; identifiedBy: Julianna Freires Barbosa; institutionCode: DZRJ; basisOfRecord: PreservedSpecimen 


\section{Family Notonectidae}

\section{Genus Buenoa Kirkaldy, 1904}

Notes: New genus record for PI.

\section{Buenoa mutabilis Truxal, 1953}

\section{Material}

a. country: Brazil; stateProvince: Piauí; municipality: Piracuruca; locality: Parque Nacional de Sete Cidades, Riacho da Bananeira; maximumElevationInMeters: 189; verbatimCoordinates: $4^{\circ} 5^{\prime} 59^{\prime \prime S}, 41^{\circ} 40^{\prime} 48^{\prime \prime W}$; samplingProtocol: Manual; verbatimEventDate: 8.ii.13; individualCount: 1; sex: male; lifeStage: adult; recordedBy: Santos, A.P.M. | Takiya, D.M.; identifiedBy: Julianna Freires Barbosa; institutionCode: DZRJ; basisOfRecord: PreservedSpecimen

Distribution: Haiti. Venezuela. Guyana. Brazil: PI!, GO, MG. Peru. Paraguay.

Notes: New species record for Northeastern Brazil.

\section{Buenoa pseudomutabilis Barbosa, Ribeiro \& Nessimian, 2010}

\section{Materials}

a. country: Brazil; stateProvince: Piauí; municipality: Piracuruca; locality: Parque Nacional de Sete Cidades, Cachoeira do Riachão; maximumElevationInMeters: 171; verbatimCoordinates: $4^{\circ} 6^{\prime} 28$ "S, 41 $4{ }^{\circ} 40^{\prime} 13 " \mathrm{~W}$; samplingProtocol: Pennsylvania light trap; verbatimEventDate: 18.iv.12; individualCount: 1; sex: male; lifeStage: adult; recordedBy: Takiya, D.M.; identifiedBy: Julianna Freires Barbosa; institutionCode: DZRJ; basisOfRecord: PreservedSpecimen

b. country: Brazil; stateProvince: Piauí; municipality: Piracuruca; locality: Parque Nacional de Sete Cidades, Cachoeira do Riachão; maximumElevationInMeters: 171; verbatimCoordinates: $4^{\circ} 6^{\prime} 28$ "S, $41^{\circ} 40^{\prime} 13$ "W; samplingProtocol: Pennsylvania light trap; verbatimEventDate: 18.iv.12; individualCount: 2; sex: female; lifeStage: adult; recordedBy: Takiya, D.M.; identifiedBy: Julianna Freires Barbosa; institutionCode: DZRJ; basisOfRecord: PreservedSpecimen

c. country: Brazil; stateProvince: Piauí; municipality: Piracuruca; locality: Parque Nacional de Sete Cidades, Cachoeira do Riachão; maximumElevationInMeters: 171; verbatimCoordinates: $4^{\circ} 6^{\prime} 28^{\prime \prime S}, 41^{\circ} 40^{\prime} 13^{\prime \prime} \mathrm{W}$; samplingProtocol: Manual; verbatimEventDate: 9.ii.13; individualCount: 2; sex: male; lifeStage: adult; recordedBy: Santos, A.P.M. | Takiya, D.M.; identifiedBy: Julianna Freires Barbosa; institutionCode: DZRJ; basisOfRecord: PreservedSpecimen

d. country: Brazil; stateProvince: Piauí; municipality: Piracuruca; locality: Parque Nacional de Sete Cidades, Cachoeira do Riachão; maximumElevationInMeters: 171; verbatimCoordinates: $4^{\circ} 6^{\prime} 28^{\prime \prime S}, 41^{\circ} 40^{\prime} 13$ "W; samplingProtocol: Manual; verbatimEventDate: 9.ii.13; individualCount: 3; sex: female; lifeStage: adult; recordedBy: Santos, A.P.M. | Takiya, D.M.; identifiedBy: Julianna Freires Barbosa; institutionCode: DZRJ; basisOfRecord: PreservedSpecimen 
e. country: Brazil; stateProvince: Piauí; municipality: Piracuruca; locality: Parque Nacional de Sete Cidades, Poço do Bananeira; maximumElevationInMeters: 189; verbatimCoordinates: $4^{\circ} 5^{\prime} 55.8^{\prime \prime S}, 41^{\circ} 40^{\prime} 33.8^{\prime \prime} \mathrm{W}$; samplingProtocol: Manual; verbatimEventDate: 8.ii.13; individualCount: 1; sex: male; lifeStage: adult; recordedBy: Santos, A.P.M. | Takiya, D.M.; identifiedBy: Julianna Freires Barbosa; institutionCode: DZRJ; basisOfRecord: PreservedSpecimen

f. country: Brazil; stateProvince: Piauí; municipality: Piracuruca; locality: Parque Nacional de Sete Cidades, Cachoeira do Riachão; maximumElevationInMeters: 171; verbatimCoordinates: $4^{\circ} 6^{\prime} 28^{\prime \prime S}, 41^{\circ} 40^{\prime} 13 " \mathrm{~W}$; sampling Protocol: Manual; verbatimEventDate: 9.ii.13; individualCount: 14; lifeStage: immature; recordedBy: Santos, A.P.M. | Takiya, D.M.; identifiedBy: Julianna Freires Barbosa; institutionCode: DZRJ; basisOfRecord: PreservedSpecimen

Distribution: Brazil: PI!, RJ.

Notes: New species record for Northeastern Brazil.

\section{Buenoa salutis Kirkaldy, 1904}

\section{Materials}

a. country: Brazil; stateProvince: Piauí; municipality: Piracuruca; locality: Parque Nacional de Sete Cidades, Alojamento; maximumElevationInMeters: 193; verbatimCoordinates: $4^{\circ}$ 5'57"S, 4142'34"W; samplingProtocol: White sheet light trap; verbatimEventDate: 18.iv.12; individualCount: 1; sex: male; lifeStage: adult; recordedBy: Takiya, D.M.; identifiedBy: Julianna Freires Barbosa; institutionCode: DZRJ; basisOfRecord: PreservedSpecimen

b. country: Brazil; stateProvince: Piauí; municipality: Piracuruca; locality: Parque Nacional de Sete Cidades, Alojamento; maximumElevationInMeters: 193; verbatimCoordinates: $4^{\circ}$ 5'57"S, 4142'34"W; samplingProtocol: White sheet light trap; verbatimEventDate: 18.iv.12; individualCount: 3; sex: female; lifeStage: adult; recordedBy: Takiya, D.M.; identifiedBy: Julianna Freires Barbosa; institutionCode: DZRJ | CZMA; basisOfRecord: PreservedSpecimen

c. country: Brazil; stateProvince: Piauí; municipality: Piracuruca; locality: Parque Nacional de Sete Cidades, Cachoeira do Riachão; maximumElevationInMeters: 171; verbatim Coordinates: $4^{\circ} 6^{\prime} 28^{\prime \prime S}, 41^{\circ} 40^{\prime} 13 " \mathrm{~W}$; samplingProtocol: Pennsylvania light trap; verbatimEventDate: 19.iv.12; individualCount: 2; sex: female; lifeStage: adult; recordedBy: Takiya, D.M.; identifiedBy: Julianna Freires Barbosa; institutionCode: CZMA; basisOfRecord: PreservedSpecimen

d. country: Brazil; stateProvince: Piauí; municipality: Piracuruca; locality: Parque Nacional de Sete Cidades, Riacho da Bananeira; maximumElevationInMeters: 189; verbatimCoordinates: $4^{\circ} 5^{\prime} 59$ "S, $41^{\circ} 40^{\prime} 48$ "W; samplingProtocol: Pennsylvania light trap; verbatimEventDate: 18.iv.12; individualCount: 2; sex: male; recordedBy: Takiya, D.M.; identifiedBy: Julianna Freires Barbosa; institutionCode: DZRJ | CZMA; basisOfRecord: PreservedSpecimen

e. $\quad$ country: Brazil; stateProvince: Piauí; municipality: Piracuruca; locality: Parque Nacional de Sete Cidades, Riacho da Bananeira; maximumElevationInMeters: 189; verbatimCoordinates: $4^{\circ} 5^{\prime} 59$ "S, 4140'48"W; samplingProtocol: Pennsylvania light trap; verbatimEventDate: 18.iv.12; individualCount: 2; sex: female; recordedBy: Takiya, D.M.; identifiedBy: Julianna Freires Barbosa; institutionCode: DZRJ | CZMA; basisOfRecord: PreservedSpecimen 
Distribution: Trinidad and Tobago. Colombia. Venezuela. Guyana. Suriname. French Guiana. Brazil: RR, PA, AM, PI!, CE, PB, PE, MT, MS, MG, SP, RJ, RS. Bolivia. Paraguay. Argentina.

Notes: New species record for PI.

\section{Buenoa tarsalis Truxal, 1953}

\section{Materials}

a. country: Brazil; stateProvince: Piauí; municipality: Piracuruca; locality: Parque Nacional de Sete Cidades, Riacho da Bananeira; maximumElevationInMeters: 189; verbatimCoordinates: 45'59"S, 4140'48"W; samplingProtocol: Manual; verbatimEventDate: 8.ii.13; individualCount: 2; sex: male; lifeStage: adult; recordedBy: Santos, A.P.M. | Takiya, D.M.; identifiedBy: Julianna Freires Barbosa; institutionCode: DZRJ; basisOfRecord: PreservedSpecimen

b. country: Brazil; stateProvince: Piauí; municipality: Piracuruca; locality: Parque Nacional de Sete Cidades, Riacho da Bananeira; maximumElevationInMeters: 189; verbatimCoordinates: $4^{\circ} 5^{\prime} 59$ "S, $41^{\circ} 40^{\prime} 48^{\prime \prime} \mathrm{W}$; samplingProtocol: Manual; verbatimEventDate: 8.ii.13; individualCount: 15; lifeStage: immature; recordedBy: Santos, A.P.M. | Takiya, D.M.; identifiedBy: Julianna Freires Barbosa; institutionCode: DZRJ; basisOfRecord: PreservedSpecimen

c. country: Brazil; stateProvince: Piauí; municipality: Piracuruca; locality: Parque Nacional de Sete Cidades, Alojamento; maximumElevationInMeters: 193; verbatimCoordinates: $4^{\circ}$

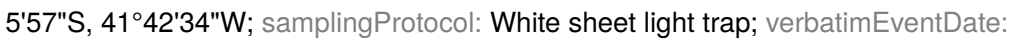
18.iv.12; individualCount: 2; sex: male; lifeStage: adult; recordedBy: Takiya, D.M.; identifiedBy: Julianna Freires Barbosa; institutionCode: DZRJ; basisOfRecord: PreservedSpecimen

d. country: Brazil; stateProvince: Piauí; municipality: Piracuruca; locality: Parque Nacional de Sete Cidades, Alojamento; maximumElevationInMeters: 193; verbatimCoordinates: $4^{\circ}$ 5'57"S, 4142'34"W; samplingProtocol: White sheet light trap; verbatimEventDate: 18.iv.12; individualCount: 3; sex: female; lifeStage: adult; recordedBy: Takiya, D.M.; identifiedBy: Julianna Freires Barbosa; institutionCode: DZRJ; basisOfRecord: PreservedSpecimen

Distribution: Brazil: PA, AM, PI!, CE, RN, PB, PE, MG, RJ.

Notes: New species record for PI.

\section{Buenoa unguis Truxal, 1953}

\section{Materials}

a. country: Brazil; stateProvince: Piauí; municipality: Piracuruca; locality: Parque Nacional de Sete Cidades, Riacho da Bananeira; maximumElevationInMeters: 189; verbatimCoordinates: $4^{\circ} 5^{\prime} 59$ "S, $41^{\circ} 40^{\prime} 48^{\prime \prime} \mathrm{W}$; samplingProtocol: Manual; verbatimEventDate: 8.ii.13; individualCount: 1; sex: male; lifeStage: adult; recordedBy: Santos, A.P.M. | Takiya, D.M.; identifiedBy: Julianna Freires Barbosa; institutionCode: DZRJ; basisOfRecord: PreservedSpecimen 
b. country: Brazil; stateProvince: Piauí; municipality: Piracuruca; locality: Parque Nacional de Sete Cidades, Riacho da Bananeira; maximumElevationInMeters: 189;

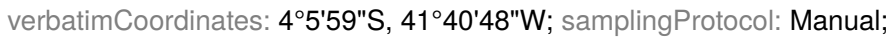
verbatimEventDate: 8.ii.13; individualCount: 9; lifeStage: immature; recordedBy: Santos, A.P.M. | Takiya, D.M.; identifiedBy: Julianna Freires Barbosa; institutionCode: DZRJ; basisOfRecord: PreservedSpecimen

c. country: Brazil; stateProvince: Piauí; municipality: Piracuruca; locality: Parque Nacional de Sete Cidades, Riacho da Bananeira; maximumElevationInMeters: 189; verbatimCoordinates: $4^{\circ} 5^{\prime} 59$ "S, $41^{\circ} 40^{\prime} 48$ "W; samplingProtocol: Pennsylvania light trap; verbatimEventDate: 18.iv.12; individualCount: 10; sex: male; lifeStage: adult; recordedBy: Takiya, D.M.; identifiedBy: Julianna Freires Barbosa; institutionCode: DZRJ; basisOfRecord: PreservedSpecimen

d. $\quad$ country: Brazil; stateProvince: Piauí; municipality: Piracuruca; locality: Parque Nacional de Sete Cidades, Riacho da Bananeira; maximumElevationInMeters: 189; verbatimCoordinates: $4^{\circ} 5^{\prime} 59$ "S, 4140'48"W; samplingProtocol: Pennsylvania light trap; verbatimEventDate: 18.iv.12; individualCount: 16; sex: female; lifeStage: adult; recordedBy: Takiya, D.M.; identifiedBy: Julianna Freires Barbosa; institutionCode: DZRJ; basisOfRecord: PreservedSpecimen

e. $\quad$ country: Brazil; stateProvince: Piauí; municipality: Piracuruca; locality: Parque Nacional de Sete Cidades, Alojamento; maximumElevationInMeters: 193; verbatimCoordinates: $4^{\circ}$ 5'57"S, 4142'34"W; samplingProtocol: White sheet light trap; verbatimEventDate: 18.iv.12; individualCount: 7; sex: male; lifeStage: adult; recordedBy: Takiya, D.M.; identifiedBy: Julianna Freires Barbosa; institutionCode: DZRJ; basisOfRecord: PreservedSpecimen

f. country: Brazil; stateProvince: Piauí; municipality: Piracuruca; locality: Parque Nacional de Sete Cidades, Alojamento; maximumElevationInMeters: 193; verbatimCoordinates: $4^{\circ}$ 5'57"S, 4142'34"W; samplingProtocol: White sheet light trap; verbatimEventDate: 18.iv.12; individualCount: 15; sex: female; lifeStage: adult; recordedBy: Takiya, D.M.; identifiedBy: Julianna Freires Barbosa; institutionCode: DZRJ; basisOfRecord: PreservedSpecimen

g. country: Brazil; stateProvince: Piauí; municipality: Piracuruca; locality: Parque Nacional de Sete Cidades, Cachoeira do Riachão; maximumElevationInMeters: 171; verbatimCoordinates: $4^{\circ} 6^{\prime} 28^{\prime \prime S}, 41^{\circ} 40^{\prime} 13$ "W; samplingProtocol: Pennsylvania light trap; verbatimEventDate: 19.iv.12; individualCount: 3; sex: male; lifeStage: adult; recordedBy: Takiya, D.M.; identifiedBy: Julianna Freires Barbosa; institutionCode: DZRJ; basisOfRecord: PreservedSpecimen

h. country: Brazil; stateProvince: Piauí; municipality: Piracuruca; locality: Parque Nacional de Sete Cidades, Cachoeira do Riachão; maximumElevationInMeters: 171; verbatimCoordinates: $4^{\circ} 6^{\prime} 28$ "S, $41^{\circ} 40^{\prime} 13^{\prime \prime} \mathrm{W}$; samplingProtocol: Pennsylvania light trap; verbatimEventDate: 19.iv.12; individualCount: 12; sex: female; lifeStage: adult; recordedBy: Takiya, D.M.; identifiedBy: Julianna Freires Barbosa; institutionCode: DZRJ; basisOfRecord: PreservedSpecimen

Distribution: Venezuela. Brazil: PA, AM, PI!, CE, RN, TO, PB, PE, MG, SP, RJ. Peru. Bolivia. Paraguay. Argentina.

Notes: New species record for PI. 


\section{Buenoa sp. 1}

\section{Materials}

a. country: Brazil; stateProvince: Piauí; municipality: Piracuruca; locality: Parque Nacional de Sete Cidades, Cachoeira do Riachão; maximumElevationInMeters: 171; verbatimCoordinates: $4^{\circ} 6^{\prime} 28^{\prime \prime S}, 41^{\circ} 40^{\prime} 13^{\prime \prime} \mathrm{W}$; samplingProtocol: Manual; verbatimEventDate: 12.ii.13; individualCount: 1; sex: male; lifeStage: adult; recordedBy: Santos, A.P.M. | Takiya, D.M.; identifiedBy: Julianna Freires Barbosa; institutionCode: DZRJ; basisOfRecord: PreservedSpecimen

b. country: Brazil; stateProvince: Piauí; municipality: Piracuruca; locality: Parque Nacional de Sete Cidades, Cachoeira do Riachão; maximumElevationInMeters: 171; verbatimCoordinates: $4^{\circ} 6^{\prime} 28$ "S, $41^{\circ} 40^{\prime} 13^{\prime \prime} \mathrm{W}$; samplingProtocol: Manual; verbatimEventDate: 12.ii.13; individualCount: 1; lifeStage: immature; recordedBy: Santos, A.P.M. | Takiya, D.M.; identifiedBy: Julianna Freires Barbosa; institutionCode: DZRJ; basisOfRecord: PreservedSpecimen

Notes: Undescribed species.

\section{Genus Martarega White, 1879}

\section{Martarega bentoi* Truxal, 1949}

\section{Materials}

a. country: Brazil; stateProvince: Piauí; municipality: Piracuruca; locality: Parque Nacional de Sete Cidades, Alojamento; maximumElevationInMeters: 193; verbatimCoordinates: $4^{\circ}$ 5'57"S, 4142'34"W; samplingProtocol: Manual; verbatimEventDate: 12.ii.13; individualCount: 1; sex: male; lifeStage: adult; recordedBy: Santos, A.P.M. | Takiya, D.M.; identifiedBy: Julianna Freires Barbosa; institutionCode: DZRJ; basisOfRecord: PreservedSpecimen

b. $\quad$ country: Brazil; stateProvince: Piauí; municipality: Piracuruca; locality: Parque Nacional de Sete Cidades, Alojamento; maximumElevationInMeters: 193; verbatimCoordinates: $4^{\circ}$ 5'57"S, 4142'34"W; samplingProtocol: Manual; verbatimEventDate: 12.ii.13; individualCount: 5; lifeStage: immature; recordedBy: Santos, A.P.M. | Takiya, D.M.; identifiedBy: Julianna Freires Barbosa; institutionCode: DZRJ; basisOfRecord: PreservedSpecimen

c. country: Brazil; stateProvince: Piauí; municipality: Piracuruca; locality: Parque Nacional de Sete Cidades, Cachoeira do Riachão; maximumElevationInMeters: 171; verbatimCoordinates: $4^{\circ} 6^{\prime} 28^{\prime \prime S}, 41^{\circ} 40^{\prime} 13^{\prime \prime} \mathrm{W}$; samplingProtocol: Manual; verbatimEventDate: 18.iv.12; individualCount: 3; sex: male; lifeStage: adult; recordedBy: Takiya, D.M.; identifiedBy: Julianna Freires Barbosa; institutionCode: DZRJ; basisOfRecord: PreservedSpecimen

d. country: Brazil; stateProvince: Piauí; municipality: Piracuruca; locality: Parque Nacional de Sete Cidades, Cachoeira do Riachão; maximumElevationInMeters: 171; verbatimCoordinates: $4^{\circ} 6^{\prime} 28^{\prime \prime} \mathrm{S}, 41^{\circ} 40^{\prime} 13^{\prime \prime} \mathrm{W}$; samplingProtocol: Manual; verbatimEventDate: 18.iv.12; individualCount: 1; sex: female; lifeStage: adult; recordedBy: Takiya, D.M.; identifiedBy: Julianna Freires Barbosa; institutionCode: DZRj; basisOfRecord: PreservedSpecimen 
Distribution: Brazil: PI, CE!, PE, MT, MG, RJ. Argentina.

\section{Martarega membranacea White, 1870}

\section{Materials}

a. country: Brazil; stateProvince: Piauí; municipality: Piracuruca; locality: Parque Nacional de Sete Cidades, Poço do Bananeira; maximumElevationInMeters: 158; verbatimCoordinates: $4^{\circ} 5^{\prime} 55.8^{\prime \prime} \mathrm{S}, 41^{\circ} 40^{\prime} 33.8^{\prime \prime} \mathrm{W}$; samplingProtocol: Manual; verbatimEventDate: 9.ii.13; individualCount: 1; sex: male; lifeStage: adult; recordedBy: Santos, A.P.M. | Takiya, D.M.; identifiedBy: Julianna Freires Barbosa; institutionCode: DZRJ; basisOfRecord: PreservedSpecimen

b. country: Brazil; stateProvince: Piauí; municipality: Piracuruca; locality: Parque Nacional de Sete Cidades, Poço do Bananeira; maximumElevationInMeters: 158; verbatimCoordinates: $4^{\circ} 5^{\prime} 55.8^{\prime \prime} \mathrm{S}, 41^{\circ} 40^{\prime} 33.8^{\prime \prime} \mathrm{W}$; samplingProtocol: Manual; verbatimEventDate: 9.ii.13; individualCount: 6; lifeStage: immature; recordedBy: Santos, A.P.M. | Takiya, D.M.; identifiedBy: Julianna Freires Barbosa; institutionCode: DZRJ; basisOfRecord: PreservedSpecimen

Distribution: Colombia. Guyana. Suriname. Brazil: PA, AM, PI!, TO, RO, MT, GO, MG, MS, SP, RJ. Ecuador. Bolivia. Argentina.

Notes: New species record for Northeastern Brazil.

\section{Genus Notonecta Linnaeus, 1758}

Notes: Genus firstly recorded from PI in Barbosa and Nessimian 2013.

\section{Notonecta disturbata Hungerford, 1926}

\section{Materials}

a. country: Brazil; stateProvince: Piauí; municipality: Piracuruca; locality: Parque Nacional de Sete Cidades, Alojamento; maximumElevationInMeters: 193; verbatimCoordinates: $4^{\circ}$ 5'57"S, 4142'34"W; samplingProtocol: Manual; verbatimEventDate: 12.ii.13; individualCount: 3; sex: male; lifeStage: adult; recordedBy: Santos, A.P.M. | Takiya, D.M.; identifiedBy: Julianna Freires Barbosa; institutionCode: DZRJ; basisOfRecord: PreservedSpecimen

b. country: Brazil; stateProvince: Piauí; municipality: Piracuruca; locality: Parque Nacional de Sete Cidades, Alojamento; maximumElevationInMeters: 193; verbatimCoordinates: $4^{\circ}$ 5'57"S, 4142'34"W; samplingProtocol: Manual; verbatimEventDate: 12.ii.13; individualCount: 1; sex: female; lifeStage: adult; recordedBy: Santos, A.P.M. | Takiya, D.M.; identifiedBy: Julianna Freires Barbosa; institutionCode: DZRJ; basisOfRecord: PreservedSpecimen

c. country: Brazil; stateProvince: Piauí; municipality: Piracuruca; locality: Parque Nacional de Sete Cidades, Cachoeira do Riachão; maximumElevationInMeters: 171; verbatimCoordinates: $4^{\circ} 6^{\prime} 28^{\prime \prime} \mathrm{S}, 41^{\circ} 40^{\prime} 13^{\prime \prime} \mathrm{W}$; samplingProtocol: Manual; verbatimEventDate: 9.ii.13; individualCount: 2; sex: male; lifeStage: adult; recordedBy: 
Santos, A.P.M. | Takiya, D.M.; identifiedBy: Julianna Freires Barbosa; institutionCode: DZRJ; basisOfRecord: PreservedSpecimen

d. country: Brazil; stateProvince: Piauí; municipality: Piracuruca; locality: Parque Nacional de Sete Cidades, Cachoeira do Riachão; maximumElevationInMeters: 171; verbatimCoordinates: $4^{\circ} 6^{\prime} 28^{\prime \prime} \mathrm{S}, 41^{\circ} 40^{\prime} 13^{\prime \prime} \mathrm{W}$; samplingProtocol: Manual; verbatimEventDate: 9.ii.13; individualCount: 1; sex: female; lifeStage: adult; recordedBy: Santos, A.P.M. | Takiya, D.M.; identifiedBy: Julianna Freires Barbosa; institutionCode: DZRJ; basisOfRecord: PreservedSpecimen

e. country: Brazil; stateProvince: Piauí; municipality: Piracuruca; locality: Parque Nacional de Sete Cidades, Cachoeira do Riachão; maximumElevationInMeters: 171; verbatimCoordinates: $4^{\circ} 6^{\prime} 28$ "S, $41^{\circ} 40^{\prime} 13^{\prime \prime} \mathrm{W}$; samplingProtocol: Manual; verbatimEventDate: 9.ii.13; individualCount: 1; lifeStage: immature; recordedBy: Santos, A.P.M. | Takiya, D.M.; identifiedBy: Julianna Freires Barbosa; institutionCode: DZRJ; basisOfRecord: PreservedSpecimen

Distribution: Brazil: PA, PI!, TO, MT, GO, MG, SP, RJ, RS. Paraguay. Argentina.

Notes: Species firstly recorded from Northeastern Brazil in Barbosa and Nessimian 2013.

\section{Family Ochteridae}

Notes: Family firstly recorded from PI in Cordeiro et al. 2014.

\section{Genus Ochterus Latreille, 1807}

Notes: Genus firstly recorded from PI in Cordeiro et al. 2014.

\section{Ochterus santosi Cordeiro \& Moreira, 2014}

\section{Materials}

a. country: Brazil; stateProvince: Piauí; municipality: Piracuruca; locality: Parque Nacional de Sete Cidades, Olha d'água dos Milagres; maximumElevationInMeters: 180; verbatimCoordinates: $4^{\circ} 5^{\prime} 31.8^{\prime \prime} \mathrm{S}, 4^{\circ} 40^{\prime} 48.2^{\prime \prime} \mathrm{W}$; samplingProtocol: Manual; verbatimEventDate: 8.ii.13; individualCount: 1; sex: male; lifeStage: adult; recordedBy: Santos, A.P.M. | Takiya, D.M.; identifiedBy: Isabelle da R. S. Cordeiro; institutionCode: CZMA; basisOfRecord: PreservedSpecimen

b. country: Brazil; stateProvince: Piauí; municipality: Piracuruca; locality: Parque Nacional de Sete Cidades, Cachoeira do Riachão; maximumElevationInMeters: 171; verbatimCoordinates: $4^{\circ} 6^{\prime} 28$ "S, 41 40'13"W; samplingProtocol: Manual; verbatimEventDate: 9.ii.13; individualCount: 1; sex: female; lifeStage: adult; recordedBy: Santos, A.P.M. | Takiya, D.M.; identifiedBy: Isabelle da R. S. Cordeiro; institutionCode: CZMA; basisOfRecord: PreservedSpecimen

Distribution: Brazil: PI.

Notes: Species described in Cordeiro et al. 2014. 


\section{Order Odonata}

\section{Suborder Anisoptera}

\section{Family Aeshnidae}

\section{Genus Coryphaeschna}

Notes: New genus record for PI.

\section{Coryphaeschna viriditas Calvert, 1952}

\section{Material}

a. $\quad$ country: Brazil; stateProvince: Piauí; municipality: Piracuruca; locality: Parque Nacional de Sete Cidades, Olho d'água Piscina do Bacuri; maximumElevationInMeters: 171; verbatimCoordinates: 46'1.2"S, 4142'38.8"W; samplingProtocol: Manual; verbatimEventDate: 9.ii.13; individualCount: 1; sex: male; lifeStage: adult; recordedBy: Santos, A.P.M. | Takiya, D.M.; identifiedBy: Ângelo Parise Pinto; institutionCode: DZRJ; basisOfRecord: PreservedSpecimen

Distribution: USA south to Panama. Trinidad and Tobago. Colombia. Venezuela. Guyana. Suriname. French Guiana. Brazil: AM/PA, PI!, PE, MT, MG, ES, RJ. Ecuador. Peru. Bolivia. Paraguay.

Notes: New species record for PI. See Fig. 27.

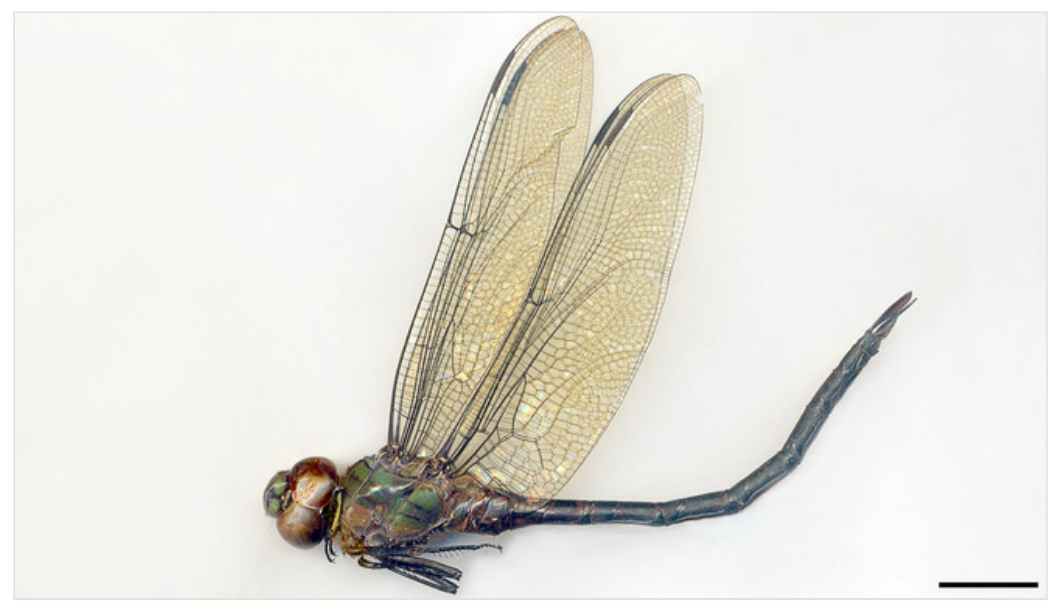

Figure 27.

Lateral habitus of a male of Coryphaeschna viriditas collected at PNSC-06 (DZRJ), representing a new state record for Piauí. Scale bar $=10 \mathrm{~mm}$. 


\section{Genus Gynacantha Rambur, 1842}

Notes: New genus record for PI.

\section{Gynacantha nervosa* Rambur, 1842}

\section{Materials}

a. $\quad$ country: Brazil; stateProvince: Piauí; municipality: Piracuruca; locality: Parque Nacional de Sete Cidades, Alojamento; maximumElevationInMeters: 193; verbatimCoordinates: $4^{\circ}$ 5'57"S, 4142'34"W; samplingProtocol: Suspended intercept trap; verbatimEventDate: 19.iv.12; individualCount: 1; sex: female; lifeStage: adult; recordedBy: Rafael, J.A. | Limeira-de-Oliveira, F. | et al.; identifiedBy: Ângelo Parise Pinto; institutionCode: CZMA; basisOfRecord: PreservedSpecimen

b. country: Brazil; stateProvince: Piauí; municipality: Piracuruca; locality: Parque Nacional de Sete Cidades, Olha d'água dos Milagres; maximumElevationInMeters: 180; verbatimCoordinates: 45'31.8"S, 41²0'48.2"W; samplingProtocol: Manual; verbatimEventDate: 8.ii.13; individualCount: 1; sex: female; lifeStage: adult; recordedBy: Santos, A.P.M. | Takiya, D.M.; identifiedBy: Ângelo Parise Pinto; institutionCode: DZRJ; basisOfRecord: PreservedSpecimen

c. country: Brazil; stateProvince: Piauí; municipality: Piracuruca; locality: Parque Nacional de Sete Cidades, Alojamento; maximumElevationInMeters: 193; verbatimCoordinates: $4^{\circ}$ 5'57"S, 4142'34"W; samplingProtocol: Manual; verbatimEventDate: 9.ii.13; individualCount: 1; sex: male; lifeStage: adult; recordedBy: Santos, A.P.M. | Takiya, D.M.; identifiedBy: Ângelo Parise Pinto; institutionCode: DZRJ; basisOfRecord: PreservedSpecimen

Distribution: USA south to Panama. Trinidad and Tobago. Colombia. Venezuela. Guyana. Suriname. French Guiana. Brazil: RR, PI!, CE, PE, MT, MG, MS, ES, RJ. Ecuador. Peru. Bolivia.

Notes: New species record for PI.

\section{Genus Triacanthagyna}

Notes: New genus record for PI.

\section{Triacanthagyna caribbea Williamson, 1923}

\section{Material}

a. $\quad$ country: Brazil; stateProvince: Piauí; municipality: Piracuruca; locality: Parque Nacional de Sete Cidades, Olho d'água Piscina do Bacuri; maximumElevationInMeters: 171; verbatimCoordinates: $4^{\circ} 6^{\prime} 1.2^{\prime \prime} \mathrm{S}, 4^{\circ} 42$ '38.8"W; samplingProtocol: White sheet light trap; verbatimEventDate: 9.ii.13; individualCount: 1; sex: female; lifeStage: adult; recordedBy: Santos, A.P.M. | Takiya, D.M.; identifiedBy: Ângelo Parise Pinto; institutionCode: DZRJ; basisOfRecord: PreservedSpecimen 
Distribution: Mexico. Belize. Guatemala. Honduras. Costa Rica. Dominican Republic. Puerto Rico. Trinidad and Tobago. Colombia. Venezuela. Suriname. French Guiana. Brazil: PA, PI!, MT, BA, ES, SP, RJ, PR. Ecuador. Peru. Bolivia.

Notes: New species record for PI.

\section{Family Libellulidae}

\section{Genus Brechmorhoga Kirby, 1889}

Notes: New genus record for PI.

\section{Brechmorhoga sp. 1}

\section{Material}

a. $\quad$ country: Brazil; stateProvince: Piauí; municipality: Piracuruca; locality: Parque Nacional de Sete Cidades, Alojamento; maximumElevationInMeters: 193; verbatimCoordinates: $4^{\circ}$ 5'57"S, 4142'34"W; samplingProtocol: Malaise intercept trap; verbatimEventDate: 19.iv.12; individualCount: 1; sex: female; lifeStage: adult; recordedBy: Rafael, J.A. | Limeira-de-Oliveira, F. | Takiya, D.M. | et al.; identifiedBy: Ângelo Parise Pinto; institutionCode: DZRJ; basisOfRecord: PreservedSpecimen

\section{Genus Diastatops Rambur, 1842}

Notes: New genus record for PI.

\section{Diastatops obscura (Fabricius, 1775)}

\section{Materials}

a. country: Brazil; stateProvince: Piauí; municipality: Piracuruca; locality: Parque Nacional de Sete Cidades, Riacho da Bananeira; maximumElevationInMeters: 189;

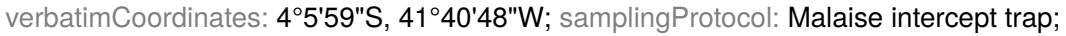
verbatimEventDate: 19.iv.12; individualCount: 1; sex: male; lifeStage: adult; recordedBy: Rafael, J.A. | Limeira-de-Oliveira, F. | Takiya, D.M. | et al.; identifiedBy: Alcimar Carvalho | Ângelo Parise Pinto; institutionCode: DZRJ; basisOfRecord: PreservedSpecimen

b. $\quad$ country: Brazil; stateProvince: Piauí; municipality: Piracuruca; locality: Parque Nacional de Sete Cidades, Riacho da Piedade; maximumElevationInMeters: 169; verbatimCoordinates: $4^{\circ} 6^{\prime} 34$ "S, $41^{\circ} 43^{\prime} 39$ "W; samplingProtocol: Malaise intercept trap; verbatimEventDate: 21.iv.12; individualCount: 1; sex: female; lifeStage: adult; recordedBy: Rafael, J.A. | Limeira-de-Oliveira, F. | Takiya, D.M. | et al.; identifiedBy: Alcimar Carvalho | Ângelo Parise Pinto; institutionCode: INPA; basisOfRecord: PreservedSpecimen

Distribution: Colombia. Venezuela. Guyana. Suriname. French Guiana. Brazil: RR, PA, AM, MA, PI!, CE, RN, TO, PE, MT, BA, MG, MS, ES, SP, RJ. Peru. Bolivia. Paraguay. Argentina. 
Notes: New species record for PI.

\section{Genus Erythrodiplax Brauer, 1868}

Notes: New genus record for PI.

\section{Erythrodiplax basalis* (Kirby, 1897)}

\section{Materials}

a. country: Brazil; stateProvince: Piauí; municipality: Piracuruca; locality: Parque Nacional de Sete Cidades, Poço do Bananeira; maximumElevationInMeters: 158; verbatimCoordinates: $4^{\circ} 5^{\prime} 55.8^{\prime \prime} \mathrm{S}, 41^{\circ} 40^{\prime} 33.8 \mathrm{\prime W}$; samplingProtocol: Manual; verbatimEventDate: 11.ii.13; individualCount: 1; sex: male; lifeStage: adult; recordedBy: Santos, A.P.M. | Takiya, D.M.; identifiedBy: Ângelo Parise Pinto; institutionCode: DZRJ; basisOfRecord: PreservedSpecimen

b. $\quad$ country: Brazil; stateProvince: Piauí; municipality: Piracuruca; locality: Parque Nacional de Sete Cidades, Alojamento; maximumElevationInMeters: 193; verbatimCoordinates: $4^{\circ}$ 5'57"S, 4142'34"W; samplingProtocol: Malaise intercept trap; verbatimEventDate: 19.iv.12; individualCount: 1; sex: female; lifeStage: adult; recordedBy: Rafael, J.A. | Limeira-de-Oliveira, F. | Takiya, D.M. | et al.; identifiedBy: Ângelo Parise Pinto; institutionCode: DZRJ; basisOfRecord: PreservedSpecimen

Distribution: Trinidad and Tobago. Colombia. Venezuela. Guyana. Suriname. French Guiana. Brazil: RR, PA, AM, MA, PI!, CE, PE, RO, BA, MT, MG, MS, ES, SP, RJ, RS. Ecuador. Peru. Bolivia. Paraguay. Argentina. Uruguay.

Notes: New species record for PI.

\section{Erythrodiplax famula (Erichson in Schomburgk, 1848)}

\section{Material}

a. country: Brazil; stateProvince: Piauí; municipality: Piracuruca; locality: Parque Nacional de Sete Cidades, Riacho da Piedade; maximumElevationInMeters: 169; verbatimCoordinates: $4^{\circ} 6^{\prime} 34$ "S, 414' 39 "W; samplingProtocol: Manual; verbatimEventDate: 19.iv.12; individualCount: 1; sex: male; lifeStage: adult; recordedBy: Takiya, D.M. | Cavichioli, R.R.; identifiedBy: Ângelo Parise Pinto; institutionCode: DZRJ; basisOfRecord: PreservedSpecimen

Distribution: Trinidad and Tobago. Colombia. Venezuela. Guyana. Suriname. French Guiana. Brazil: PA, AM, PI!, PE, GO, MG, ES, SP, RJ. Peru. Argentina.

Notes: New species record for PI. 


\section{Erythrodiplax fusca* (Rambur, 1842)}

\section{Material}

a. country: Brazil; stateProvince: Piauí; municipality: Piracuruca; locality: Parque Nacional de Sete Cidades, Alojamento; maximumElevationInMeters: 193; verbatimCoordinates: $4^{\circ}$ 5'57"S, 4142'34"W; samplingProtocol: Malaise intercept trap; verbatimEventDate: 19.iv.12; individualCount: 1; sex: female; lifeStage: adult; recordedBy: Rafael, J.A. | Limeira-de-Oliveira, F. | Takiya, D.M. | et al.; identifiedBy: Ângelo Parise Pinto; institutionCode: DZRJ; basisOfRecord: PreservedSpecimen

Distribution: USA south to Panama. Trinidad and Tobago. Colombia. Venezuela. Guyana. Suriname. French Guiana. Brazil: PA, AM, MA, PI!, CE, RN, PE, MT, RO, BA, GO, MG, MS, ES, SP, RJ, SC, RS. Ecuador. Peru. Bolivia. Paraguay. Argentina. Uruguay.

Notes: New species record for PI.

\section{Erythrodiplax umbrata (Linnaeus, 1758)}

\section{Materials}

a. country: Brazil; stateProvince: Piauí; municipality: Piracuruca; locality: Parque Nacional de Sete Cidades, Riacho da Bananeira; maximumElevationInMeters: 189; verbatimCoordinates: $4^{\circ} 5^{\prime} 59^{\prime \prime} \mathrm{S}, 41^{\circ} 40^{\prime} 48^{\prime \prime} \mathrm{W}$; sampling Protocol: Manual; verbatimEventDate: 13.ii.13; individualCount: 1; sex: male; lifeStage: adult; recordedBy: Santos, A.P.M. | Takiya, D.M.; identifiedBy: Ângelo Parise Pinto; institutionCode: CZMA; basisOfRecord: PreservedSpecimen

b. country: Brazil; stateProvince: Piauí; municipality: Piracuruca; locality: Parque Nacional de Sete Cidades, Riacho da Bananeira; maximumElevationInMeters: 189; verbatimCoordinates: $4^{\circ} 5^{\prime} 59^{\prime \prime S}, 41^{\circ} 40^{\prime} 48$ "W; samplingProtocol: Manual; verbatimEventDate: 13.ii.13; individualCount: 1; sex: male; lifeStage: adult; recordedBy: Santos, A.P.M. | Takiya, D.M.; identifiedBy: Ângelo Parise Pinto; institutionCode: DZRJ; basisOfRecord: PreservedSpecimen

c. country: Brazil; stateProvince: Piauí; municipality: Piracuruca; locality: Parque Nacional de Sete Cidades, Riacho da Piedade; maximumElevationInMeters: 169;

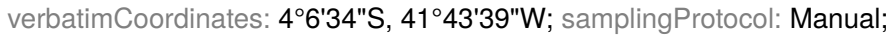
verbatimEventDate: 19.iv.12; individualCount: 4; sex: male; lifeStage: adult; recordedBy: Takiya, D.M. | Cavichioli, R.R.; identifiedBy: Alcimar Carvalho | Ângelo Parise Pinto; institutionCode: DZRJ; basisOfRecord: PreservedSpecimen

d. country: Brazil; stateProvince: Piauí; municipality: Piracuruca; locality: Parque Nacional de Sete Cidades, Olha d'água dos Milagres; maximumElevationInMeters: 180; verbatimCoordinates: $4^{\circ} 5^{\prime} 31.8^{\prime \prime S}, 41^{\circ} 40^{\prime} 48.2^{\prime \prime W}$; samplingProtocol: White sheet light trap; verbatimEventDate: 9.ii.13; individualCount: 1; sex: female; lifeStage: adult; recordedBy: Santos, A.P.M. | Takiya, D.M.; identifiedBy: Ângelo Parise Pinto; institutionCode: DZRJ; basisOfRecord: PreservedSpecimen

Distribution: USA south to Panama. Trinidad and Tobago. Colombia. Venezuela. Guyana. Suriname. French Guyana. Brazil: RR, PA, AM, PI!, CE, PE, MT, RO, BA, MG, MS, ES, SP, RJ, RS. Ecuador. Peru. Bolivia. Paraguay. Argentina. Uruguay. 
Notes: New species record for PI.

\section{Genus Orthemis Hagen, 1861}

Notes: New genus record for PI.

\section{Orthemis aequilibris [red morph] Calvert, 1909}

\section{Materials}

a. country: Brazil; stateProvince: Piauí; municipality: Piracuruca; locality: Parque Nacional de Sete Cidades, Riacho da Bananeira; maximumElevationInMeters: 189; verbatimCoordinates: $4^{\circ} 5^{\prime} 59$ "S, 4140'48"W; samplingProtocol: Manual; verbatimEventDate: 13.ii.13; individualCount: 1; sex: male; lifeStage: adult; recordedBy: Santos, A.P.M. | Takiya, D.M.; identifiedBy: Ângelo Parise Pinto; institutionCode: DZRJ; basisOfRecord: PreservedSpecimen

b. country: Brazil; stateProvince: Piauí; municipality: Piracuruca; locality: Parque Nacional de Sete Cidades, Riacho da Bananeira; maximumElevationInMeters: 189; verbatimCoordinates: 45'59"S, 4140'48"W; samplingProtocol: Manual; verbatimEventDate: 13.ii.13; individualCount: 1; sex: female; lifeStage: adult; recordedBy: Santos, A.P.M. | Takiya, D.M.; identifiedBy: Ângelo Parise Pinto; institutionCode: CZMA; basisOfRecord: PreservedSpecimen

c. country: Brazil; stateProvince: Piauí; municipality: Piracuruca; locality: Parque Nacional de Sete Cidades, Riacho da Bananeira; maximumElevationInMeters: 189; verbatimCoordinates: $4^{\circ} 5^{\prime} 59^{\prime \prime S}$, 4140'48"W; samplingProtocol: Manual; verbatimEventDate: 8.ii.13; individualCount: 1; sex: male; lifeStage: adult; recordedBy: Santos, A.P.M. | Takiya, D.M.; identifiedBy: Ângelo Parise Pinto; institutionCode: DZRJ; basisOfRecord: PreservedSpecimen

Distribution: Costa Rica. Panama. Colombia. Venezuela. Guyana. Suriname. Frech Guiana. Brazil: PA, AM, PI!, CE, BA, MG, MS, ES, RJ. Ecuador. Peru. Bolivia. Paraguay. Argentina.

Notes: New species record for PI.

\section{Orthemis flavopicta Kirby, 1889}

\section{Material}

a. country: Brazil; stateProvince: Piauí; municipality: Piracuruca; locality: Parque Nacional de Sete Cidades, Olho d'água Piscina do Bacuri; maximumElevationInMeters: 171; verbatimCoordinates: $4^{\circ} 6^{\prime} 1.2^{\prime \prime} \mathrm{S}, 41^{\circ} 42^{\prime} 38.8^{\prime \prime} \mathrm{W}$; samplingProtocol: Pennsylvania light trap; verbatimEventDate: 8.ii.13; individualCount: 1; sex: female; lifeStage: adult; recordedBy: Santos, A.P.M. | Takiya, D.M.; identifiedBy: Ângelo Parise Pinto; institutionCode: DZRJ; basisOfRecord: PreservedSpecimen

Distribution: Venezuela. Brazil: PA, PI!, CE, RO, BA, GO. Bolivia.

Notes: New species record for PI. See Fig. 28. 


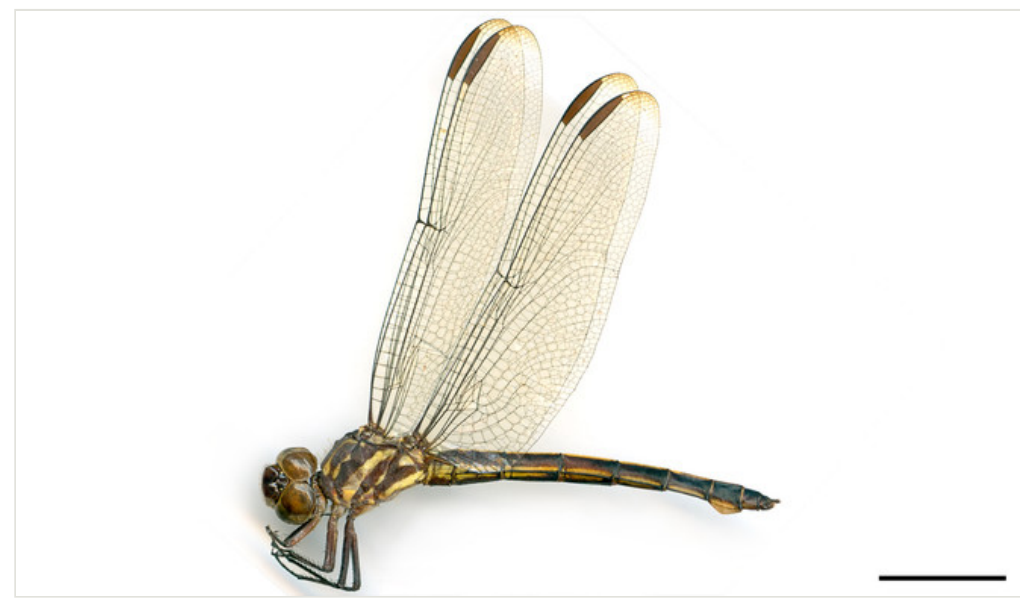

Figure 28.

Lateral habitus of a female of Orthemis flavopicta collected at PNSC-06 (DZRJ), representing a new state record for Piauí. Scale bar $=10 \mathrm{~mm}$.

\section{Genus Pantala Hagen, 1861}

Notes: New genus record for PI.

\section{Pantala flavescens (Fabricius, 1798)}

\section{Materials}

a. country: Brazil; stateProvince: Piauí; municipality: Piracuruca; locality: Parque Nacional de Sete Cidades, Olho d'água Piscina do Bacuri; maximumElevationInMeters: 171; verbatimCoordinates: $4^{\circ} 6^{\prime} 1.2^{\prime \prime} \mathrm{S}, 41^{\circ} 42^{\prime} 38.8^{\prime \prime} \mathrm{W}$; samplingProtocol: Manual; verbatimEventDate: 10.ii.13; individualCount: 1; sex: male; lifeStage: adult; recordedBy: Santos, A.P.M. | Takiya, D.M.; identifiedBy: Ângelo Parise Pinto; institutionCode: DZRJ; basisOfRecord: PreservedSpecimen

b. $\quad$ country: Brazil; stateProvince: Piauí; municipality: Piracuruca; locality: Parque Nacional de Sete Cidades, Olho d'água Piscina do Bacuri; maximumElevationInMeters: 171; verbatimCoordinates: $4^{\circ} 6^{\prime} 1.2^{\prime \prime} \mathrm{S}, 41^{\circ} 42^{\prime} 38.8^{\prime \prime} \mathrm{W}$; samplingProtocol: Manual; verbatimEventDate: 10.ii.13; individualCount: 1; sex: female; lifeStage: adult; recordedBy: Santos, A.P.M. | Takiya, D.M.; identifiedBy: Ângelo Parise Pinto; institutionCode: CZMA; basisOfRecord: PreservedSpecimen

c. country: Brazil; stateProvince: Piauí; municipality: Piracuruca; locality: Parque Nacional de Sete Cidades, Riacho da Bananeira; maximumElevationInMeters: 189; verbatimCoordinates: $4^{\circ} 5^{\prime} 59^{\prime \prime} \mathrm{S}, 41^{\circ} 40^{\prime} 48^{\prime \prime} \mathrm{W}$; sampling Protocol: Manual; verbatimEventDate: 13.ii.13; individualCount: 1; sex: male; lifeStage: adult; recordedBy: Santos, A.P.M. | Takiya, D.M.; identifiedBy: Ângelo Parise Pinto; institutionCode: CZMA; basisOfRecord: PreservedSpecimen

d. country: Brazil; stateProvince: Piauí; municipality: Piracuruca; locality: Parque Nacional de Sete Cidades, Riacho da Bananeira; maximumElevationInMeters: 189; verbatimCoordinates: $4^{\circ} 5^{\prime} 59^{\prime \prime S}$, 414ㅇ'48"W; samplingProtocol: Manual; 
verbatimEventDate: 13.ii.13; individualCount: 1; sex: male; lifeStage: adult; recordedBy: Santos, A.P.M. | Takiya, D.M.; identifiedBy: Ângelo Parise Pinto; institutionCode: CZMA; basisOfRecord: PreservedSpecimen

e. country: Brazil; stateProvince: Piauí; municipality: Piracuruca; locality: Parque Nacional de Sete Cidades, Riacho da Bananeira; maximumElevationInMeters: 189; verbatimCoordinates: $4^{\circ} 5^{\prime} 59^{\prime \prime S}, 41^{\circ} 40^{\prime} 48^{\prime \prime} \mathrm{W}$; samplingProtocol: Manual; verbatimEventDate: 13.ii.13; individualCount: 1; sex: male; lifeStage: adult; recordedBy: Santos, A.P.M. | Takiya, D.M.; identifiedBy: Ângelo Parise Pinto; institutionCode: DZRJ; basisOfRecord: PreservedSpecimen

f. country: Brazil; stateProvince: Piauí; municipality: Piracuruca; locality: Parque Nacional de Sete Cidades, Riacho da Bananeira; maximumElevationInMeters: 189; verbatimCoordinates: $4^{\circ} 5^{\prime} 59^{\prime \prime} \mathrm{S}, 41^{\circ} 40^{\prime} 48^{\prime \prime} \mathrm{W}$; sampling Protocol: Manual; verbatimEventDate: 13.ii.13; individualCount: 1; sex: male; lifeStage: adult; recordedBy: Santos, A.P.M. | Takiya, D.M.; identifiedBy: Ângelo Parise Pinto; institutionCode: DZRJ; basisOfRecord: PreservedSpecimen

g. country: Brazil; stateProvince: Piauí; municipality: Piracuruca; locality: Parque Nacional de Sete Cidades, Riacho da Bananeira; maximumElevationInMeters: 189; verbatimCoordinates: $4^{\circ} 5^{\prime} 59 " \mathrm{~S}, 41^{\circ} 40^{\prime} 48^{\prime \prime} \mathrm{W}$; samplingProtocol: Manual; verbatimEventDate: 13.ii.13; individualCount: 1; sex: male; lifeStage: adult; recordedBy: Santos, A.P.M. | Takiya, D.M.; identifiedBy: Ângelo Parise Pinto; institutionCode: DZRJ; basisOfRecord: PreservedSpecimen

h. country: Brazil; stateProvince: Piauí; municipality: Piracuruca; locality: Parque Nacional de Sete Cidades, Riacho da Bananeira; maximumElevationInMeters: 189; verbatimCoordinates: 45'59"S, 4140'48"W; samplingProtocol: Manual; verbatimEventDate: 13.ii.13; individualCount: 1; sex: male; lifeStage: adult; recordedBy: Santos, A.P.M. | Takiya, D.M.; identifiedBy: Ângelo Parise Pinto; institutionCode: DZRJ; basisOfRecord: PreservedSpecimen

i. country: Brazil; stateProvince: Piauí; municipality: Piracuruca; locality: Parque Nacional de Sete Cidades, Alojamento; maximumElevationInMeters: 193; verbatimCoordinates: $4^{\circ}$ 5'57"S, 4142'34"W; samplingProtocol: Manual; verbatimEventDate: 13.ii.13; individualCount: 1; sex: male; lifeStage: adult; recordedBy: Santos, A.P.M. | Takiya, D.M.; identifiedBy: Ângelo Parise Pinto; institutionCode: DZRJ; basisOfRecord:

PreservedSpecimen

j. country: Brazil; stateProvince: Piauí; municipality: Piracuruca; locality: Parque Nacional de Sete Cidades, Riacho da Piedade; maximumElevationInMeters: 169; verbatimCoordinates: $4^{\circ} 6^{\prime} 34$ "S, $41^{\circ} 43^{\prime} 39$ "W; samplingProtocol: Manual; verbatimEventDate: 19.iv.12; individualCount: 1; sex: male; lifeStage: adult; recordedBy: Takiya, D.M. | Cavichioli, R.R.; identifiedBy: Alcimar Carvalho | Ângelo Parise Pinto; institutionCode: DZRJ; basisOfRecord: PreservedSpecimen

k. country: Brazil; stateProvince: Piauí; municipality: Piracuruca; locality: Parque Nacional de Sete Cidades, Riacho da Bananeira; maximumElevationInMeters: 189; verbatimCoordinates: $4^{\circ} 5^{\prime} 59^{\prime \prime S}, 41^{\circ} 40^{\prime} 48$ "W; samplingProtocol: Manual; verbatimEventDate: 8.ii.13; individualCount: 1; sex: male; lifeStage: adult; recordedBy: Santos, A.P.M. | Takiya, D.M.; identifiedBy: Ângelo Parise Pinto; institutionCode: DZRJ; basisOfRecord: PreservedSpecimen

I. country: Brazil; stateProvince: Piauí; municipality: Piracuruca; locality: Parque Nacional de Sete Cidades, Olho d'água Piscina do Bacuri; maximumElevationInMeters: 171; verbatimCoordinates: $4^{\circ} 6^{\prime} 1.2^{\prime \prime} \mathrm{S}, 41^{\circ} 42^{\prime} 38.8^{\prime \prime} \mathrm{W}$; samplingProtocol: Manual; verbatimEventDate: 9.ii.13; individualCount: 1; sex: female; lifeStage: adult; recordedBy: 
Santos, A.P.M. | Takiya, D.M.; identifiedBy: Ângelo Parise Pinto; institutionCode: CZMA; basisOfRecord: PreservedSpecimen

Distribution: Pantropical. Widespread in the Americas. Brazil: RR, PA, AM, MA, PI!, CE, TO, PE, MT, RO, BA, MG, MS, ES, SP, RJ, PR, SC, RS, also in Fernando de Noronha and Trindade e Martim Vaz archipelagoes.

Notes: New species record for PI.

\section{Genus Perithemis Hagen, 1861}

\section{Perithemis lais (Perty, 1834)}

\section{Materials}

a. country: Brazil; stateProvince: Piauí; municipality: Piracuruca; locality: Parque Nacional de Sete Cidades, Alojamento; maximumElevationInMeters: 193; verbatimCoordinates: $4^{\circ}$ 5'57"S, 4142'34"W; samplingProtocol: Malaise intercept trap; verbatimEventDate: 19.iv.12; individualCount: 1; sex: male; lifeStage: adult; recordedBy: Rafael, J.A. | Limeirade-Oliveira, F. | Takiya, D.M. | et al.; identifiedBy: Ângelo Parise Pinto; institutionCode: DZRJ; basisOfRecord: PreservedSpecimen

b. country: Brazil; stateProvince: Piauí; municipality: Piracuruca; locality: Parque Nacional de Sete Cidades, Olha d'água dos Milagres; maximumElevationInMeters: 180; verbatimCoordinates: $4^{\circ} 5^{\prime} 31.8^{\prime \prime} \mathrm{S}, 4^{\circ} 40^{\prime} 48.2^{\prime \prime} \mathrm{W}$; samplingProtocol: White sheet light trap; verbatimEventDate: 9.ii.13; individualCount: 1; sex: male; lifeStage: adult; recordedBy: Santos, A.P.M. | Takiya, D.M.; identifiedBy: Ângelo Parise Pinto; institutionCode: DZRJ; basisOfRecord: PreservedSpecimen

Distribution: Trinidad and Tobago. Colombia. Venezuela. Guyana. Suriname. French Guiana. Brazil: RR, PA, AM, MA, PI!, PE, MT, RO, MS, ES, SP. Ecuador. Peru. Bolivia. Argentina.

Notes: New species record for PI.

\section{Suborder Zygoptera}

\section{Family Calopterygidae}

\section{Genus Hetaerina Hagen in Selys, 1853}

Notes: New genus record for PI.

\section{Hetaerina sp. 1}

\section{Material}

a. country: Brazil; stateProvince: Piauí; municipality: Piracuruca; locality: Parque Nacional de Sete Cidades, Alojamento; maximumElevationInMeters: 193; verbatimCoordinates: $4^{\circ}$ 
5'57"S, 4142'34"W; samplingProtocol: Malaise intercept trap; verbatimEventDate: 19.iv.12; individualCount: 1; sex: female; lifeStage: adult; recordedBy: Rafael, J.A. | Limeira-de-Oliveira, F. | Takiya, D.M. | et al.; identifiedBy: Ângelo Parise Pinto; institutionCode: DZRJ; basisOfRecord: PreservedSpecimen

\section{Family Coenagrionidae}

\section{Genus Acanthagrion Selys, 1876}

Notes: New genus record for PI.

\section{Acanthagrion jessei Leonard, 1977}

\section{Materials}

a. country: Brazil; stateProvince: Piauí; municipality: Piracuruca; locality: Parque Nacional de Sete Cidades, Alojamento; maximumElevationInMeters: 193; verbatimCoordinates: $4^{\circ}$ 5'57"S, 4142'34"W; samplingProtocol: Malaise intercept trap; verbatimEventDate: 16.xii.12; individualCount: 1; sex: male; lifeStage: adult; recordedBy: Limeira-de-Oliveira, F. | Pinto Júnior, J.S.; identifiedBy: Ângelo Parise Pinto; institutionCode: DZRJ; basisOfRecord: PreservedSpecimen

b. $\quad$ country: Brazil; stateProvince: Piauí; municipality: Piracuruca; locality: Parque Nacional de Sete Cidades, Riacho da Bananeira; maximumElevationInMeters: 189; verbatimCoordinates: $4^{\circ} 5^{\prime} 59$ "S, $41^{\circ} 40^{\prime} 48^{\prime \prime}$; sampling Protocol: Malaise intercept trap; verbatimEventDate: 19.iv.12; individualCount: 1; sex: female; lifeStage: adult; recordedBy: Rafael, J.A. | Limeira-de-Oliveira, F. | Takiya, D.M. | et al.; identifiedBy: Ângelo Parise Pinto; institutionCode: DZRJ; basisOfRecord: PreservedSpecimen

c. country: Brazil; stateProvince: Piauí; municipality: Piracuruca; locality: Parque Nacional de Sete Cidades, Alojamento; maximumElevationInMeters: 193; verbatimCoordinates: $4^{\circ}$ 5'57"S, 4142'34"W; samplingProtocol: Suspended intercept trap; verbatimEventDate: 19.iv.12; individualCount: 1; sex: male; lifeStage: adult; recordedBy: Rafael, J.A. | Limeirade-Oliveira, F. | et al.; identifiedBy: Ângelo Parise Pinto; institutionCode: DZRJ; basisOfRecord: PreservedSpecimen

d. country: Brazil; stateProvince: Piauí; municipality: Piracuruca; locality: Parque Nacional de Sete Cidades, Riacho da Piedade; maximumElevationInMeters: 169; verbatimCoordinates: $4^{\circ} 6^{\prime} 34$ "S, $41^{\circ} 43^{\prime} 39$ "W; samplingProtocol: Malaise intercept trap; verbatimEventDate: 21.iv.12; individualCount: 2; sex: male; lifeStage: adult; recordedBy: Rafael, J.A. | Limeira-de-Oliveira, F. | Takiya, D.M. | et al.; identifiedBy: Ângelo Parise Pinto; institutionCode: DZRJ; basisOfRecord: PreservedSpecimen

e. $\quad$ country: Brazil; stateProvince: Piauí; municipality: Piracuruca; locality: Parque Nacional de Sete Cidades, Riacho da Piedade; maximumElevationInMeters: 169; verbatimCoordinates: $4^{\circ} 6^{\prime} 34$ "S, $41^{\circ} 43^{\prime} 39$ "W; sampling Protocol: Malaise intercept trap; verbatimEventDate: 21.iv.12; individualCount: 3; sex: female; lifeStage: adult; recordedBy: Rafael, J.A. | Limeira-de-Oliveira, F. | Takiya, D.M. | et al.; identifiedBy: Ângelo Parise Pinto; institutionCode: DZRJ; basisOfRecord: PreservedSpecimen

Distribution: Brazil: PI!, MT, RO, MS. Ecuador.

Notes: New species record for PI. 


\section{Acanthagrion truncatum Selys, 1876}

\section{Materials}

a. country: Brazil; stateProvince: Piauí; municipality: Piracuruca; locality: Parque Nacional de Sete Cidades, Poço do Bananeira; maximumElevationInMeters: 158; verbatimCoordinates: $4^{\circ} 5^{\prime} 55.8^{\prime \prime} \mathrm{S}, 41^{\circ} 40^{\prime} 33.8^{\prime \prime} \mathrm{W}$; samplingProtocol: Manual; verbatimEventDate: 11.ii.13; individualCount: 1; sex: male; lifeStage: adult; recordedBy: Santos, A.P.M. | Takiya, D.M.; identifiedBy: Ângelo Parise Pinto; institutionCode: DZRJ; basisOfRecord: PreservedSpecimen

b. country: Brazil; stateProvince: Piauí; municipality: Piracuruca; locality: Parque Nacional de Sete Cidades, Poço do Bananeira; maximumElevationInMeters: 158; verbatimCoordinates: $4^{\circ} 5^{\prime} 55.8^{\prime \prime} \mathrm{S}, 41^{\circ} 40^{\prime} 33.8^{\prime \prime} \mathrm{W}$; samplingProtocol: Manual; verbatimEventDate: 9.ii.13; individualCount: 1; sex: male; lifeStage: adult; recordedBy: Santos, A.P.M. | Takiya, D.M.; identifiedBy: Ângelo Parise Pinto; institutionCode: DZRJ; basisOfRecord: PreservedSpecimen

Distribution: Venezuela. Guyana. Brazil: PI!, TO, MT, GO, MG, MS, SP.

Notes: New species record for PI.

\section{Genus Argia Rambur, 1842}

Notes: New genus record for PI.

\section{Argia tinctipennis* Selys, 1865}

\section{Material}

a. country: Brazil; stateProvince: Piauí; municipality: Piracuruca; locality: Parque Nacional de Sete Cidades, Cachoeira do Riachão; maximumElevationInMeters: 171; verbatimCoordinates: $4^{\circ} 6^{\prime} 28$ "S, $41^{\circ} 40^{\prime} 13$ "W; samplingProtocol: Suspended intercept trap; verbatimEventDate: 17.ii.13; individualCount: 1; sex: male; lifeStage: adult; recordedBy: Rafael, J.A. | Limeira-de-Oliveira, F. | et al.; identifiedBy: Rosser W. Garrison; institutionCode: DZRJ; basisOfRecord: PreservedSpecimen

Distribution: Brazil: AM, PI!, CE!, MT, GO, MS. Peru.

Notes: New species record for Northeastern Brazil.

\section{Genus Epipleoneura Williamson, 1915}

Notes: New genus record for $\mathrm{PI}$.

\section{Epipleoneura metallica Rácenis, 1955}

\section{Material}

a. country: Brazil; stateProvince: Piauí; municipality: Piracuruca; locality: Parque Nacional de Sete Cidades, Alojamento; maximumElevationInMeters: 193; verbatimCoordinates: $4^{\circ}$ 
5'57"S, 4142'34"W; samplingProtocol: Malaise intercept trap; verbatimEventDate: 19.iv.12; individualCount: 1; sex: female; lifeStage: adult; recordedBy: Rafael, J.A. | Limeira-de-Oliveira, F. | Takiya, D.M. | et al.; identifiedBy: Ângelo Parise Pinto; institutionCode: DZRJ; basisOfRecord: PreservedSpecimen

Distribution: Colombia. Venezuela. Brazil: PA, AM, MA, PI!, CE, TO, PE, MT, RO, BA, GO, MG, DF, ES.

Notes: New species record for PI.

\section{Genus Ischnura}

Notes: New genus record for PI.

\section{Ischnura capreolus (Hagen, 1861)}

\section{Materials}

a. country: Brazil; stateProvince: Piauí; municipality: Piracuruca; locality: Parque Nacional de Sete Cidades, Poço do Bananeira; maximumElevationInMeters: 158; verbatimCoordinates: $4^{\circ} 5^{\prime} 55.8^{\prime \prime} \mathrm{S}, 41^{\circ} 40^{\prime} 33.8^{\prime \prime} \mathrm{W}$; samplingProtocol: Manual; verbatimEventDate: 11.ii.13; individualCount: 1; sex: male; lifeStage: adult; recordedBy: Santos, A.P.M. | Takiya, D.M.; identifiedBy: Ângelo Parise Pinto; institutionCode: CZMA; basisOfRecord: PreservedSpecimen

b. country: Brazil; stateProvince: Piauí; municipality: Piracuruca; locality: Parque Nacional de Sete Cidades, Poço do Bananeira; maximumElevationInMeters: 158; verbatimCoordinates: $4^{\circ} 5^{\prime} 55.8^{\prime \prime} \mathrm{S}, 4^{\circ} 40^{\prime} 33.8^{\prime \prime} \mathrm{W}$; samplingProtocol: Manual; verbatimEventDate: 11.ii.13; individualCount: 1; sex: male; lifeStage: adult; recordedBy: Santos, A.P.M. | Takiya, D.M.; identifiedBy: Ângelo Parise Pinto; institutionCode: DZRJ; basisOfRecord: PreservedSpecimen

Distribution: Mexico south to Panama. Trinidad and Tobago. Colombia. Venezuela. Guyana. Suriname. French Guiana. Brazil: PA, AM, PI!, CE, PB, PE, MT, RO, BA, MG, MS, ES, SP, RJ, RS. Ecuador. Peru. Bolivia. Paraguay. Argentina.

Notes: New species record for PI.

\section{Genus Neoneura Selys, 1860}

Notes: New genus record for PI.

\section{Neoneura sylvatica Hagen in Selys, 1886}

\section{Material}

a. country: Brazil; stateProvince: Piauí; municipality: Piracuruca; locality: Parque Nacional de Sete Cidades, Alojamento; maximumElevationInMeters: 193; verbatimCoordinates: $4^{\circ}$ 5'57"S, 4142'34"W; samplingProtocol: Malaise intercept trap; verbatimEventDate: 16.xii.12; individualCount: 1; sex: female; lifeStage: adult; recordedBy: Limeira-de- 
Oliveira, F. | Pinto Júnior, J.S.; identifiedBy: Ângelo Parise Pinto; institutionCode: DZRJ; basisOfRecord: PreservedSpecimen

Distribution: Colombia. Venezuela. Suriname. French Guiana. Brazil: MA, PI!, CE, TO, PE, MT, RO, BA, GO, MG, MS, SP, RJ, PR. Bolivia. Argentina.

Notes: New species record for PI.

\section{Genus Oxyagrion Selys, 1876}

Notes: New genus record for PI.

\section{Oxyagrion sp. 1 [0. basale group]}

\section{Material}

a. country: Brazil; stateProvince: Piauí; municipality: Piracuruca; locality: Parque Nacional de Sete Cidades, Alojamento; maximumElevationInMeters: 193; verbatimCoordinates: $4^{\circ}$ 5'57"S, 41 42'34"W; samplingProtocol: Suspended intercept trap; verbatimEventDate: 19.iv.12; individualCount: 1; sex: male; lifeStage: adult; recordedBy: Rafael, J.A. | Limeirade-Oliveira, F. | et al.; identifiedBy: Ângelo Parise Pinto; institutionCode: DZRJ; basisOfRecord: PreservedSpecimen

Notes: Likely an undescribed species.

\section{Order Plecoptera}

Notes: New order record for PI.

\section{Family Perlidae}

Notes: New family record for PI.

\section{Genus Anacroneuria Klapálek, 1909}

Notes: New genus record for PI.

\section{Anacroneuria calori* Duarte \& Lecci, 2016}

\section{Materials}

a. country: Brazil; stateProvince: Piauí; municipality: Piracuruca; locality: Parque Nacional de Sete Cidades, Cachoeira do Riachão; maximumElevationInMeters: 171; verbatimCoordinates: $4^{\circ} 6^{\prime} 28^{\prime \prime S}, 41^{\circ} 40^{\prime} 13 " \mathrm{~W}$; samplingProtocol: Pennsylvania light trap; verbatimEventDate: 19.iv.12; individualCount: 1; sex: female; lifeStage: adult; recordedBy: Takiya, D.M.; identifiedBy: Fernanda Avelino-Capistrano; institutionCode: DZRJ; basisOfRecord: PreservedSpecimen

b. country: Brazil; stateProvince: Piauí; municipality: Piracuruca; locality: Parque Nacional de Sete Cidades, Cachoeira do Riachão; maximumElevationlnMeters: 171; 
verbatimCoordinates: $4^{\circ} 6^{\prime} 28^{\prime \prime S}, 41^{\circ} 40^{\prime} 13^{\prime \prime W}$; samplingProtocol: Pennsylvania light trap; verbatimEventDate: 19.iv.12; individualCount: 1; sex: male; lifeStage: adult; recordedBy: Takiya, D.M.; identifiedBy: Fernanda Avelino-Capistrano; institutionCode: DZRJ; basisOfRecord: PreservedSpecimen

c. country: Brazil; stateProvince: Piauí; municipality: Piracuruca; locality: Parque Nacional de Sete Cidades, Cachoeira do Riachão; maximumElevationInMeters: 171; verbatimCoordinates: $4^{\circ} 6^{\prime} 28^{\prime \prime} \mathrm{S}, 41^{\circ} 40^{\prime} 13^{\prime \prime} \mathrm{W}$; samplingProtocol: Pennsylvania light trap; verbatimEventDate: 20.iv.12; individualCount: 1; sex: male; lifeStage: adult; recordedBy: Takiya, D.M. | Rafael, J.A.; identifiedBy: Fernanda Avelino-Capistrano; institutionCode: DZRJ; basisOfRecord: PreservedSpecimen

d. country: Brazil; stateProvince: Piauí; municipality: Piracuruca; locality: Parque Nacional de Sete Cidades, Cachoeira do Riachão; maximumElevationInMeters: 171; verbatimCoordinates: $4^{\circ} 6^{\prime} 28^{\prime \prime} \mathrm{S}, 41^{\circ} 40^{\prime} 13^{\prime \prime} \mathrm{W}$; samplingProtocol: Pennsylvania light trap; verbatimEventDate: 20.iv.12; individualCount: 1; sex: male; lifeStage: adult; recordedBy: Takiya, D.M. | Rafael, J.A.; identifiedBy: Fernanda Avelino-Capistrano; institutionCode: DZRJ; basisOfRecord: PreservedSpecimen

Distribution: Brazil: CE, PI!.

Notes: New species record for PI. See Fig. 25.

\section{Order Trichoptera}

\section{Family Ecnomidae}

Notes: New family record for PI.

\section{Genus Austrotinodes Schmid, 1955}

Notes: New genus record for PI.

\section{Austrotinodes paraguayensis* Flint, 1983}

\section{Material}

a. country: Brazil; stateProvince: Piauí; municipality: Piracuruca; locality: Parque Nacional de Sete Cidades, Riacho da Bananeira; maximumElevationInMeters: 189; verbatimCoordinates: $4^{\circ} 5^{\prime} 59$ "S, $41^{\circ} 40^{\prime} 48^{\prime \prime} \mathrm{W}$; samplingProtocol: Malaise intercept trap; verbatimEventDate: 19.iv.12; individualCount: 1; sex: male; lifeStage: adult; recordedBy: Rafael, J.A. | Limeira-de-Oliveira, F. | Takiya, D.M. | et al.; identifiedBy: Wagner Rafael Maciel de Souza; institutionCode: DZRJ; basisOfRecord: PreservedSpecimen

Distribution: Brazil: PI!, CE!, MG. Paraguay.

Notes: New species record for Northeastern Brazil. 


\section{Family Helicopsychidae}

\section{Genus Helicopsyche von Siebold, 1856}

\section{Helicopsyche (Feropsyche) vergelana* Ross, 1956}

\section{Materials}

a. country: Brazil; stateProvince: Piauí; municipality: Piracuruca; locality: Parque Nacional de Sete Cidades, Cachoeira do Riachão; maximumElevationInMeters: 171; verbatimCoordinates: $4^{\circ} 6^{\prime} 28$ "S, $41^{\circ} 40^{\prime} 13^{\prime \prime} \mathrm{W}$; samplingProtocol: Pennsylvania light trap; verbatimEventDate: 18.iv.12; individualCount: 1; sex: male; lifeStage: adult; recordedBy: Takiya, D.M.; identifiedBy: Allan Paulo Moreira dos Santos; institutionCode: DZRJ; basisOfRecord: PreservedSpecimen

b. $\quad$ country: Brazil; stateProvince: Piauí; municipality: Piracuruca; locality: Parque Nacional de Sete Cidades, Cachoeira do Riachão; maximumElevationInMeters: 171; verbatimCoordinates: $4^{\circ} 6^{\prime} 28^{\prime \prime} \mathrm{S}, 41^{\circ} 40^{\prime} 13^{\prime \prime} \mathrm{W}$; samplingProtocol: Pennsylvania light trap; verbatimEventDate: 18.iv.12; individualCount: 1; sex: female; lifeStage: adult; recordedBy: Takiya, D.M.; identifiedBy: Allan Paulo Moreira dos Santos; institutionCode: DZRJ; basisOfRecord: PreservedSpecimen

c. country: Brazil; stateProvince: Piauí; municipality: Piracuruca; locality: Parque Nacional de Sete Cidades, Riacho da Piedade; maximumElevationInMeters: 169; verbatimCoordinates: $4^{\circ} 6^{\prime} 34^{\prime \prime S}, 41^{\circ} 43^{\prime} 39$ "W; samplingProtocol: Malaise intercept trap; verbatimEventDate: 18.iv.12; individualCount: 1; sex: male; lifeStage: adult; recordedBy: Rafael, J.A. | Limeira-de-Oliveira, F. | Takiya, D.M. | et al.; identifiedBy: Allan Paulo Moreira dos Santos; institutionCode: DZRJ; basisOfRecord: PreservedSpecimen

d. country: Brazil; stateProvince: Piauí; municipality: Piracuruca; locality: Parque Nacional de Sete Cidades, Riacho da Piedade; maximumElevationInMeters: 169; verbatimCoordinates: $4^{\circ} 6^{\prime} 34^{\prime \prime} \mathrm{S}, 41^{\circ} 43^{\prime} 39 " \mathrm{~W}$; samplingProtocol: Malaise intercept trap; verbatimEventDate: 21.iv.12; individualCount: 3; sex: male; lifeStage: adult; recordedBy: Rafael, J.A. | Limeira-de-Oliveira, F. | Takiya, D.M. | et al.; identifiedBy: Allan Paulo Moreira dos Santos; institutionCode: DZRJ; basisOfRecord: PreservedSpecimen

e. $\quad$ country: Brazil; stateProvince: Piauí; municipality: Piracuruca; locality: Parque Nacional de Sete Cidades, Riacho da Piedade; maximumElevationInMeters: 169; verbatimCoordinates: $4^{\circ} 6^{\prime} 34$ "S, 41 ${ }^{\circ} 43^{\prime} 39$ "W; samplingProtocol: Malaise intercept trap; verbatimEventDate: 21.iv.12; individualCount: 13; sex: male; lifeStage: adult; recordedBy: Rafael, J.A. | Limeira-de-Oliveira, F. | Takiya, D.M. | et al.; identifiedBy: Allan Paulo Moreira dos Santos; institutionCode: DZRJ; basisOfRecord: PreservedSpecimen

f. country: Brazil; stateProvince: Piauí; municipality: Piracuruca; locality: Parque Nacional de Sete Cidades, Riacho da Piedade; maximumElevationInMeters: 169; verbatimCoordinates: $4^{\circ} 6^{\prime} 34$ "S, $41^{\circ} 43^{\prime} 39$ "W; samplingProtocol: Malaise intercept trap; verbatimEventDate: 21.iv.12; individualCount: 2; sex: female; lifeStage: adult; recordedBy: Rafael, J.A. | Limeira-de-Oliveira, F. | Takiya, D.M. | et al.; identifiedBy: Allan Paulo Moreira dos Santos; institutionCode: DZRJ; basisOfRecord: PreservedSpecimen

Distribution: Mexico. Belize. Guatemala. Honduras. Costa Rica. Panama. Trinidad and Tobago. Venezuela. Suriname. Brazil: PI!, CE!, PE. Peru.

Notes: New species record for PI. 


\section{Family Hydropsychidae}

Notes: New family record for PI.

\section{Genus Leptonema Guérin, 1843}

Notes: New genus record for PI.

\section{Leptonema viridianum* Navás, 1916}

\section{Materials}

a. country: Brazil; stateProvince: Piauí; municipality: Piracuruca; locality: Parque Nacional de Sete Cidades, Riacho da Piedade; maximumElevationInMeters: 169; verbatimCoordinates: $4^{\circ} 6^{\prime} 34^{\prime \prime S}, 41^{\circ} 43^{\prime} 39$ "W; samplingProtocol: Malaise intercept trap; verbatimEventDate: 18.iv.12; individualCount: 2; sex: male; lifeStage: adult; recordedBy: Rafael, J.A. | Limeira-de-Oliveira, F. | Takiya, D.M. | et al.; identifiedBy: Allan Paulo Moreira dos Santos; institutionCode: DZRJ; basisOfRecord: PreservedSpecimen

b. $\quad$ country: Brazil; stateProvince: Piauí; municipality: Piracuruca; locality: Parque Nacional de Sete Cidades, Riacho da Piedade; maximumElevationInMeters: 169; verbatimCoordinates: $4^{\circ} 6^{\prime} 34$ "S, $41^{\circ} 43^{\prime} 39$ "W; sampling Protocol: Malaise intercept trap; verbatimEventDate: 21.iv.12; individualCount: 3; sex: male; lifeStage: adult; recordedBy: Rafael, J.A. | Limeira-de-Oliveira, F. | Takiya, D.M. | et al.; identifiedBy: Allan Paulo Moreira dos Santos; institutionCode: DZRJ; basisOfRecord: PreservedSpecimen

c. country: Brazil; stateProvince: Piauí; municipality: Piracuruca; locality: Parque Nacional de Sete Cidades, Riacho da Piedade; maximumElevationInMeters: 169; verbatimCoordinates: $4^{\circ} 6^{\prime} 34$ "S, $41^{\circ} 43^{\prime} 39$ "W; samplingProtocol: Malaise intercept trap; verbatimEventDate: 21.iv.12; individualCount: 4; sex: female; lifeStage: adult; recordedBy: Rafael, J.A. | Limeira-de-Oliveira, F. | Takiya, D.M. | et al.; identifiedBy: Allan Paulo Moreira dos Santos; institutionCode: DZRJ; basisOfRecord: PreservedSpecimen

Distribution: Colombia. Venezuela. Guyana. Brazil: PA, PI!, CE, GO, MG, DF, ES, RJ. Ecuador. Peru. Bolivia. Paraguay. Argentina.

Notes: New species record for PI.

\section{Genus Macronema Pictet, 1836}

Notes: New genus record for PI.

\section{Macronema sp. 1}

\section{Material}

a. country: Brazil; stateProvince: Piauí; municipality: Piracuruca; locality: Parque Nacional de Sete Cidades, Riacho da Piedade; maximumElevationInMeters: 169; verbatimCoordinates: $4^{\circ} 6^{\prime} 34$ "S, $41^{\circ} 43^{\prime} 39$ "W; sampling Protocol: Malaise intercept trap; verbatimEventDate: 18.iv.12; individualCount: 0; sex: female; lifeStage: adult; recordedBy: 
Rafael, J.A. | Limeira-de-Oliveira, F. | Takiya, D.M. | et al.; identifiedBy: Allan Paulo Moreira dos Santos; institutionCode: DZRJ; basisOfRecord: PreservedSpecimen

\section{Genus Macrostemum Kolenati, 1859}

Notes: New genus record for PI.

\section{Macrostemum ulmeri* (Banks, 1913)}

\section{Materials}

a. country: Brazil; stateProvince: Piauí; municipality: Piracuruca; locality: Parque Nacional de Sete Cidades, Cachoeira do Riachão; maximumElevationInMeters: 171; verbatimCoordinates: $4^{\circ} 6^{\prime} 28^{\prime \prime} \mathrm{S}, 41^{\circ} 40^{\prime} 13^{\prime \prime W}$; samplingProtocol: Malaise intercept trap; verbatimEventDate: 18.iv.12; individualCount: 1; sex: male; lifeStage: adult; recordedBy: Rafael, J.A. | Limeira-de-Oliveira, F. | Takiya, D.M. | et al.; identifiedBy: Allan Paulo Moreira dos Santos; institutionCode: DZRJ; basisOfRecord: PreservedSpecimen

b. country: Brazil; stateProvince: Piauí; municipality: Piracuruca; locality: Parque Nacional de Sete Cidades, Cachoeira do Riachão; maximumElevationInMeters: 171; verbatimCoordinates: $4^{\circ} 6^{\prime} 28^{\prime \prime} \mathrm{S}, 41^{\circ} 40^{\prime} 13^{\prime \prime} \mathrm{W}$; samplingProtocol: Malaise intercept trap; verbatimEventDate: 18.iv.12; individualCount: 1; sex: female; lifeStage: adult; recordedBy: Rafael, J.A. | Limeira-de-Oliveira, F. | Takiya, D.M. | et al.; identifiedBy: Allan Paulo Moreira dos Santos; institutionCode: DZRJ; basisOfRecord: PreservedSpecimen

c. country: Brazil; stateProvince: Piauí; municipality: Piracuruca; locality: Parque Nacional de Sete Cidades, Cachoeira do Riachão; maximumElevationInMeters: 171; verbatimCoordinates: $4^{\circ} 6^{\prime} 28^{\prime \prime S}, 41^{\circ} 40^{\prime} 13$ "W; samplingProtocol: Malaise intercept trap; verbatimEventDate: 18.iv.12; individualCount: 1; sex: male; lifeStage: adult; recordedBy: Rafael, J.A. | Limeira-de-Oliveira, F. | Takiya, D.M. | et al.; identifiedBy: Allan Paulo Moreira dos Santos; institutionCode: DZRJ; basisOfRecord: PreservedSpecimen

d. country: Brazil; stateProvince: Piauí; municipality: Piracuruca; locality: Parque Nacional de Sete Cidades, Riacho da Piedade; maximumElevationInMeters: 169; verbatimCoordinates: $4^{\circ} 6^{\prime} 34$ "S, $41^{\circ} 43^{\prime} 39$ "W; sampling Protocol: Malaise intercept trap; verbatimEventDate: 18.iv.12; individualCount: 4; sex: male; lifeStage: adult; recordedBy: Rafael, J.A. | Limeira-de-Oliveira, F. | Takiya, D.M. | et al.; identifiedBy: Allan Paulo Moreira dos Santos; institutionCode: DZRJ; basisOfRecord: PreservedSpecimen

e. country: Brazil; stateProvince: Piauí; municipality: Piracuruca; locality: Parque Nacional de Sete Cidades, Riacho da Piedade; maximumElevationInMeters: 169; verbatimCoordinates: $4^{\circ} 6^{\prime} 34$ "S, $41^{\circ} 43^{\prime} 39 " \mathrm{~W}$; samplingProtocol: Malaise intercept trap; verbatimEventDate: 18.iv.12; individualCount: 6; sex: female; lifeStage: adult; recordedBy: Rafael, J.A. | Limeira-de-Oliveira, F. | Takiya, D.M. | et al.; identifiedBy: Allan Paulo Moreira dos Santos; institutionCode: DZRJ; basisOfRecord: PreservedSpecimen

f. country: Brazil; stateProvince: Piauí; municipality: Piracuruca; locality: Parque Nacional de Sete Cidades, Riacho da Piedade; maximumElevationInMeters: 169; verbatimCoordinates: $4^{\circ} 6^{\prime} 34$ "S, 41 ${ }^{\circ} 43^{\prime} 39$ "W; samplingProtocol: Malaise intercept trap; verbatimEventDate: 18.iv.12; individualCount: 22; sex: male; lifeStage: adult; recordedBy: Rafael, J.A. | Limeira-de-Oliveira, F. | Takiya, D.M. | et al.; identifiedBy: Allan Paulo Moreira dos Santos; institutionCode: DZRJ; basisOfRecord: PreservedSpecimen

g. country: Brazil; stateProvince: Piauí; municipality: Piracuruca; locality: Parque Nacional de Sete Cidades, Riacho da Piedade; maximumElevationInMeters: 169; verbatimCoordinates: $4^{\circ} 6^{\prime} 34$ "S, $41^{\circ} 43^{\prime} 39$ "W; samplingProtocol: Malaise intercept trap; 
verbatimEventDate: 18.iv.12; individualCount: 22; sex: female; lifeStage: adult; recordedBy: Rafael, J.A. | Limeira-de-Oliveira, F. | Takiya, D.M. | et al.; identifiedBy: Allan Paulo Moreira dos Santos; institutionCode: DZRJ; basisOfRecord: PreservedSpecimen

h. country: Brazil; stateProvince: Piauí; municipality: Piracuruca; locality: Parque Nacional de Sete Cidades, Riacho da Piedade; maximumElevationInMeters: 169;

verbatimCoordinates: $4^{\circ} 6^{\prime} 34$ "S, $41^{\circ} 43^{\prime} 39 " \mathrm{~W}$; samplingProtocol: Malaise intercept trap; verbatimEventDate: 18.iv.12; individualCount: 22; sex: male; lifeStage: adult; recordedBy: Rafael, J.A. | Limeira-de-Oliveira, F. | Takiya, D.M. | et al.; identifiedBy: Allan Paulo Moreira dos Santos; institutionCode: DZRJ; basisOfRecord: PreservedSpecimen

i. country: Brazil; stateProvince: Piauí; municipality: Piracuruca; locality: Parque Nacional de Sete Cidades, Riacho da Piedade; maximumElevationInMeters: 169; verbatimCoordinates: $4^{\circ} 6^{\prime} 34$ "S, $41^{\circ} 43^{\prime} 39$ "W; samplingProtocol: Malaise intercept trap; verbatimEventDate: 18.iv.12; individualCount: 22; sex: female; lifeStage: adult; recordedBy: Rafael, J.A. | Limeira-de-Oliveira, F. | Takiya, D.M. | et al.; identifiedBy: Allan Paulo Moreira dos Santos; institutionCode: DZRJ; basisOfRecord: PreservedSpecimen

j. country: Brazil; stateProvince: Piauí; municipality: Piracuruca; locality: Parque Nacional de Sete Cidades, Riacho da Bananeira; maximumElevationInMeters: 189;

verbatimCoordinates: $4^{\circ} 5^{\prime} 59$ "S, $41^{\circ} 40^{\prime} 48^{\prime \prime W}$; samplingProtocol: Malaise intercept trap; verbatimEventDate: 19.iv.12; individualCount: 2; sex: male; lifeStage: adult; recordedBy: Rafael, J.A. | Limeira-de-Oliveira, F. | Takiya, D.M. | et al.; identifiedBy: Allan Paulo Moreira dos Santos; institutionCode: DZRJ; basisOfRecord: PreservedSpecimen

k. country: Brazil; stateProvince: Piauí; municipality: Piracuruca; locality: Parque Nacional de Sete Cidades, Riacho da Bananeira; maximumElevationInMeters: 189; verbatimCoordinates: $4^{\circ} 5^{\prime} 59$ "S, 4140'48"W; samplingProtocol: Malaise intercept trap; verbatimEventDate: 19.iv.12; individualCount: 6; sex: female; lifeStage: adult; recordedBy: Rafael, J.A. | Limeira-de-Oliveira, F. | Takiya, D.M. | et al.; identifiedBy: Allan Paulo Moreira dos Santos; institutionCode: DZRJ; basisOfRecord: PreservedSpecimen

I. country: Brazil; stateProvince: Piauí; municipality: Piracuruca; locality: Parque Nacional de Sete Cidades, Riacho da Piedade; maximumElevationInMeters: 169; verbatimCoordinates: $4^{\circ} 6^{\prime} 34$ "S, $41^{\circ} 43^{\prime} 39$ "W; samplingProtocol: Pennsylvania light trap; verbatimEventDate: 19.iv.12; individualCount: 1; sex: female; lifeStage: adult; recordedBy: Takiya, D.M.; identifiedBy: Allan Paulo Moreira dos Santos; institutionCode: INPA; basisOfRecord: PreservedSpecimen

m. country: Brazil; stateProvince: Piauí; municipality: Piracuruca; locality: Parque Nacional de Sete Cidades, Riacho da Piedade; maximumElevationInMeters: 169; verbatimCoordinates: $4^{\circ} 6^{\prime} 34$ "S, $41^{\circ} 43^{\prime} 39 " \mathrm{~W}$; samplingProtocol: Malaise intercept trap; verbatimEventDate: 21.iv.12; individualCount: 50; sex: male; lifeStage: adult; recordedBy: Rafael, J.A. | Limeira-de-Oliveira, F. | Takiya, D.M. | et al.; identifiedBy: Allan Paulo Moreira dos Santos; institutionCode: DZRJ; basisOfRecord: PreservedSpecimen

n. country: Brazil; stateProvince: Piauí; municipality: Piracuruca; locality: Parque Nacional de Sete Cidades, Riacho da Piedade; maximumElevationInMeters: 169; verbatimCoordinates: $4^{\circ} 6^{\prime} 34^{\prime \prime S}, 41^{\circ} 43^{\prime} 39 " \mathrm{~W}$; samplingProtocol: Malaise intercept trap; verbatimEventDate: 21.iv.12; individualCount: 33; sex: female; lifeStage: adult; recordedBy: Rafael, J.A. | Limeira-de-Oliveira, F. | Takiya, D.M. | et al.; identifiedBy: Allan Paulo Moreira dos Santos; institutionCode: DZRJ; basisOfRecord: PreservedSpecimen

o. country: Brazil; stateProvince: Piauí; municipality: Piracuruca; locality: Parque Nacional de Sete Cidades, Riacho da Piedade; maximumElevationInMeters: 169; verbatimCoordinates: $4^{\circ} 6{ }^{\prime} 34$ "S, $41^{\circ} 43^{\prime} 39 \mathrm{WW}$; samplingProtocol: Malaise intercept trap; verbatimEventDate: 21.iv.12; individualCount: 1; sex: male; lifeStage: adult; recordedBy: 
Rafael, J.A. | Limeira-de-Oliveira, F. | Takiya, D.M. | et al.; identifiedBy: Allan Paulo Moreira dos Santos; institutionCode: DZRJ; basisOfRecord: PreservedSpecimen

Distribution: Honduras. Costa Rica. Panama. Colombia. Suriname. Brazil: PA, AM, PI!, CE!, MT, AC, RO, SP. Ecuador. Peru.

Notes: New species record for PI.

\section{Genus Smicridea McLachlan, 1871}

Notes: New genus record for PI.

\section{Smicridea (Rhyacophylax) sp. 1}

\section{Materials}

a. country: Brazil; stateProvince: Piauí; municipality: Piracuruca; locality: Parque Nacional de Sete Cidades, Riacho da Piedade; maximumElevationInMeters: 169; verbatimCoordinates: $4^{\circ} 6^{\prime} 34$ "S, 4143'39"W; samplingProtocol: Pennsylvania light trap; verbatimEventDate: 19.iv.12; individualCount: 3; sex: male; lifeStage: adult; recordedBy: Takiya, D.M.; identifiedBy: Allan Paulo Moreira dos Santos; institutionCode: DZRJ; basisOfRecord: PreservedSpecimen

b. country: Brazil; stateProvince: Piauí; municipality: Piracuruca; locality: Parque Nacional de Sete Cidades, Riacho da Piedade; maximumElevationInMeters: 169; verbatimCoordinates: $4^{\circ} 6^{\prime} 34$ "S, $41^{\circ} 43^{\prime} 39$ "W; samplingProtocol: Pennsylvania light trap; verbatimEventDate: 19.iv.12; individualCount: 3; sex: female; lifeStage: adult; recordedBy: Takiya, D.M.; identifiedBy: Allan Paulo Moreira dos Santos; institutionCode: DZRJ; basisOfRecord: PreservedSpecimen

c. country: Brazil; stateProvince: Piauí; municipality: Piracuruca; locality: Parque Nacional de Sete Cidades, Riacho da Piedade; maximumElevationInMeters: 169; verbatimCoordinates: $4^{\circ} 6^{\prime} 34$ "S, 41 ${ }^{\circ} 43^{\prime} 39$ "W; samplingProtocol: Malaise intercept trap; verbatimEventDate: 21.iv.12; individualCount: 1; sex: male; lifeStage: adult; recordedBy: Rafael, J.A. | Limeira-de-Oliveira, F. | Takiya, D.M. | et al.; identifiedBy: Allan Paulo Moreira dos Santos; institutionCode: DZRJ; basisOfRecord: PreservedSpecimen

d. country: Brazil; stateProvince: Piauí; municipality: Piracuruca; locality: Parque Nacional de Sete Cidades, Riacho da Piedade; maximumElevationInMeters: 169; verbatimCoordinates: $4^{\circ} 6^{\prime} 34^{\prime \prime S}, 41^{\circ} 43^{\prime} 39 " \mathrm{~W}$; samplingProtocol: Malaise intercept trap; verbatimEventDate: 21.iv.12; individualCount: 1; sex: female; lifeStage: adult; recordedBy: Rafael, J.A. | Limeira-de-Oliveira, F. | Takiya, D.M. | et al.; identifiedBy: Allan Paulo Moreira dos Santos; institutionCode: DZRJ; basisOfRecord: PreservedSpecimen

\section{Smicridea (Smicridea) bivittata* (Hagen, 1861)}

\section{Material}

a. country: Brazil; stateProvince: Piauí; municipality: Piracuruca; locality: Parque Nacional de Sete Cidades, Cachoeira do Riachão; maximumElevationInMeters: 171; verbatimCoordinates: $4^{\circ} 6^{\prime} 28^{\prime \prime S}, 41^{\circ} 40^{\prime} 13 " \mathrm{~W}$; samplingProtocol: Pennsylvania light trap; verbatimEventDate: 19.iv.12; individualCount: 1; sex: male; lifeStage: adult; recordedBy: 
Takiya, D.M.; identifiedBy: Allan Paulo Moreira dos Santos; institutionCode: DZRJ; basisOfRecord: PreservedSpecimen

Distribution: Mexico. Guatemala. Honduras. El Salvador. Costa Rica. Trinidad and Tobago. Venezuela. Suriname. Brazil: PA, PI!, CE!, MG, SP. Ecuador.

Notes: New species record for Northeastern Brazil.

\section{Smicridea (Smicridea) sp. 1}

\section{Materials}

a. $\quad$ country: Brazil; stateProvince: Piauí; municipality: Piracuruca; locality: Parque Nacional de Sete Cidades, Cachoeira do Riachão; maximumElevationInMeters: 171; verbatimCoordinates: $4^{\circ} 6^{\prime} 28^{\prime \prime S}, 41^{\circ} 40^{\prime} 13 " \mathrm{~W}$; samplingProtocol: Pennsylvania light trap; verbatimEventDate: 18.iv.12; individualCount: 2; sex: male; lifeStage: adult; recordedBy: Takiya, D.M.; identifiedBy: Allan Paulo Moreira dos Santos; institutionCode: DZRJ; basisOfRecord: PreservedSpecimen

b. country: Brazil; stateProvince: Piauí; municipality: Piracuruca; locality: Parque Nacional de Sete Cidades, Cachoeira do Riachão; maximumElevationInMeters: 171; verbatimCoordinates: $4^{\circ} 6^{\prime} 28^{\prime \prime} \mathrm{S}, 41^{\circ} 40^{\prime} 13^{\prime \prime} \mathrm{W}$; samplingProtocol: Malaise intercept trap; verbatimEventDate: 18.iv.12; individualCount: 1; sex: male; lifeStage: adult; recordedBy: Rafael, J.A. | Limeira-de-Oliveira, F. | Takiya, D.M. | et al.; identifiedBy: Allan Paulo Moreira dos Santos; institutionCode: DZRJ; basisOfRecord: PreservedSpecimen

c. country: Brazil; stateProvince: Piauí; municipality: Piracuruca; locality: Parque Nacional de Sete Cidades, Riacho da Piedade; maximumElevationInMeters: 169; verbatimCoordinates: $4^{\circ} 6^{\prime} 34$ "S, $41^{\circ} 43^{\prime} 39$ "W; sampling Protocol: Malaise intercept trap; verbatimEventDate: 18.iv.12; individualCount: 17; sex: male; lifeStage: adult; recordedBy: Rafael, J.A. | Limeira-de-Oliveira, F. | Takiya, D.M. | et al.; identifiedBy: Allan Paulo Moreira dos Santos; institutionCode: DZRJ; basisOfRecord: PreservedSpecimen

d. country: Brazil; stateProvince: Piauí; municipality: Piracuruca; locality: Parque Nacional de Sete Cidades, Riacho da Piedade; maximumElevationInMeters: 169; verbatimCoordinates: $4^{\circ} 6^{\prime} 34^{\prime \prime S}, 41^{\circ} 43^{\prime} 39 " \mathrm{~W}$; samplingProtocol: Malaise intercept trap; verbatimEventDate: 18.iv.12; individualCount: 17; sex: male; lifeStage: adult; recordedBy: Rafael, J.A. | Limeira-de-Oliveira, F. | Takiya, D.M. | et al.; identifiedBy: Allan Paulo Moreira dos Santos; institutionCode: DZRJ; basisOfRecord: PreservedSpecimen

e. country: Brazil; stateProvince: Piauí; municipality: Piracuruca; locality: Parque Nacional de Sete Cidades, Cachoeira do Riachão; maximumElevationInMeters: 171; verbatimCoordinates: $4^{\circ} 6^{\prime} 28^{\prime \prime S}, 41^{\circ} 40^{\prime} 13^{\prime \prime W}$; samplingProtocol: Pennsylvania light trap; verbatimEventDate: 20.iv.12; individualCount: 1; sex: male; lifeStage: adult; recordedBy: Takiya, D.M. | Rafael, J.A.; identifiedBy: Allan Paulo Moreira dos Santos; institutionCode: DZRJ; basisOfRecord: PreservedSpecimen

f. country: Brazil; stateProvince: Piauí; municipality: Piracuruca; locality: Parque Nacional de Sete Cidades, Alojamento; maximumElevationInMeters: 193; verbatimCoordinates: $4^{\circ}$ 5'57"S, $41^{\circ} 42^{\prime} 34$ "W; samplingProtocol: White sheet light trap; verbatimEventDate: 20.iv.12; individualCount: 1; sex: male; lifeStage: adult; recordedBy: Takiya, D.M.; identifiedBy: Allan Paulo Moreira dos Santos; institutionCode: DZRJ; basisOfRecord: PreservedSpecimen

g. country: Brazil; stateProvince: Piauí; municipality: Piracuruca; locality: Parque Nacional de Sete Cidades, Riacho da Piedade; maximumElevationInMeters: 169; 
verbatimCoordinates: $4^{\circ} 6^{\prime} 34^{\prime \prime} \mathrm{S}, 41^{\circ} 43^{\prime} 39$ "W; samplingProtocol: Malaise intercept trap; verbatimEventDate: 21.iv.12; individualCount: 26; sex: male; lifeStage: adult; recordedBy: Rafael, J.A. | Limeira-de-Oliveira, F. | Takiya, D.M. | et al.; identifiedBy: Allan Paulo Moreira dos Santos; institutionCode: DZRJ; basisOfRecord: PreservedSpecimen

h. country: Brazil; stateProvince: Piauí; municipality: Piracuruca; locality: Parque Nacional de Sete Cidades, Riacho da Piedade; maximumElevationInMeters: 169; verbatimCoordinates: $4^{\circ} 6^{\prime} 34$ "S, $41^{\circ} 43^{\prime} 39$ "W; sampling Protocol: Malaise intercept trap; verbatimEventDate: 21.iv.12; individualCount: 27; sex: male; lifeStage: adult; recordedBy: Rafael, J.A. | Limeira-de-Oliveira, F. | Takiya, D.M. | et al.; identifiedBy: Allan Paulo Moreira dos Santos; institutionCode: DZRJ; basisOfRecord: PreservedSpecimen

i. country: Brazil; stateProvince: Piauí; municipality: Piracuruca; locality: Parque Nacional de Sete Cidades, Riacho da Piedade; maximumElevationInMeters: 169; verbatimCoordinates: $4^{\circ} 6^{\prime} 34^{\prime \prime S}, 41^{\circ} 43^{\prime} 39 " \mathrm{~W}$; samplingProtocol: Malaise intercept trap; verbatimEventDate: 21.iv.12; individualCount: 28; sex: female; lifeStage: adult; recordedBy: Rafael, J.A. | Limeira-de-Oliveira, F. | Takiya, D.M. | et al.; identifiedBy: Allan Paulo Moreira dos Santos; institutionCode: DZRJ; basisOfRecord: PreservedSpecimen

Notes: Undescribed species.

\section{Smicridea (Smicridea) sp. 2*}

\section{Materials}

a. $\quad$ country: Brazil; stateProvince: Piauí; municipality: Piracuruca; locality: Parque Nacional de Sete Cidades, Cachoeira do Riachão; maximumElevationInMeters: 171; verbatimCoordinates: $4^{\circ} 6^{\prime} 28$ 'S, $41^{\circ} 40^{\prime} 13$ "W; samplingProtocol: Pennsylvania light trap; verbatimEventDate: 18.iv.12; individualCount: 2; sex: male; lifeStage: adult; recordedBy: Takiya, D.M.; identifiedBy: Allan Paulo Moreira dos Santos; institutionCode: DZRJ; basisOfRecord: PreservedSpecimen

b. country: Brazil; stateProvince: Piauí; municipality: Piracuruca; locality: Parque Nacional de Sete Cidades, Cachoeira do Riachão; maximumElevationInMeters: 171; verbatimCoordinates: $4^{\circ} 6^{\prime} 28^{\prime \prime} \mathrm{S}, 41^{\circ} 40^{\prime} 13^{\prime \prime W}$; samplingProtocol: Malaise intercept trap; verbatimEventDate: 18.iv.12; individualCount: 3; sex: male; lifeStage: adult; recordedBy: Rafael, J.A. | Limeira-de-Oliveira, F. | Takiya, D.M. | et al.; identifiedBy: Allan Paulo Moreira dos Santos; institutionCode: DZRJ; basisOfRecord: PreservedSpecimen

c. country: Brazil; stateProvince: Piauí; municipality: Piracuruca; locality: Parque Nacional de Sete Cidades, Riacho da Piedade; maximumElevationInMeters: 169; verbatimCoordinates: $4^{\circ} 6^{\prime} 34$ "S, $41^{\circ} 43^{\prime} 39$ "W; samplingProtocol: Malaise intercept trap; verbatimEventDate: 18.iv.12; individualCount: 2; sex: male; lifeStage: adult; recordedBy: Rafael, J.A. | Limeira-de-Oliveira, F. | Takiya, D.M. | et al.; identifiedBy: Allan Paulo Moreira dos Santos; institutionCode: DZRJ; basisOfRecord: PreservedSpecimen

d. country: Brazil; stateProvince: Piauí; municipality: Piracuruca; locality: Parque Nacional de Sete Cidades, Riacho da Piedade; maximumElevationInMeters: 169; verbatimCoordinates: $4^{\circ} 6^{\prime} 34$ "S, 41 ${ }^{\circ} 43^{\prime} 39$ "W; samplingProtocol: Malaise intercept trap; verbatimEventDate: 18.iv.12; individualCount: 3; sex: male; lifeStage: adult; recordedBy: Rafael, J.A. | Limeira-de-Oliveira, F. | Takiya, D.M. | et al.; identifiedBy: Allan Paulo Moreira dos Santos; institutionCode: DZRJ; basisOfRecord: PreservedSpecimen

e. $\quad$ country: Brazil; stateProvince: Piauí; municipality: Piracuruca; locality: Parque Nacional de Sete Cidades, Riacho da Bananeira; maximumElevationInMeters: 189; verbatimCoordinates: $4^{\circ} 5^{\prime} 59$ "S, $41^{\circ} 40^{\prime} 48^{\prime \prime} \mathrm{W}$; samplingProtocol: Malaise intercept trap; 
verbatimEventDate: 19.iv.12; individualCount: 2; sex: male; lifeStage: adult; recordedBy: Rafael, J.A. | Limeira-de-Oliveira, F. | Takiya, D.M. | et al.; identifiedBy: Allan Paulo Moreira dos Santos; institutionCode: DZRJ; basisOfRecord: PreservedSpecimen

f. country: Brazil; stateProvince: Piauí; municipality: Piracuruca; locality: Parque Nacional de Sete Cidades, Riacho da Piedade; maximumElevationInMeters: 169;

verbatimCoordinates: $4^{\circ} 6^{\prime} 34$ "S, $41^{\circ} 43^{\prime} 39 " \mathrm{~W}$; samplingProtocol: Malaise intercept trap; verbatimEventDate: 21.iv.12; individualCount: 2; sex: male; lifeStage: adult; recordedBy: Rafael, J.A. | Limeira-de-Oliveira, F. | Takiya, D.M. | et al.; identifiedBy: Allan Paulo Moreira dos Santos; institutionCode: DZRJ; basisOfRecord: PreservedSpecimen

Notes: Undescribed species.

\section{Family Hydroptilidae}

Notes: Family firstly recorded from PI in Souza et al. 2014a.

\section{Genus Betrichia Mosely, 1939}

Notes: Genus firstly recorded from PI in Souza et al. 2016b.

\section{Betrichia nhundiaquara Souza, Santos \& Takiya, 2016}

\section{Material}

a. country: Brazil; stateProvince: Piauí; municipality: Piracuruca; locality: Parque Nacional de Sete Cidades, Riacho da Piedade; maximumElevationInMeters: 169; verbatimCoordinates: $4^{\circ} 6^{\prime} 34$ "S, $41^{\circ} 43^{\prime} 39 " \mathrm{~W}$; samplingProtocol: Malaise intercept trap; verbatimEventDate: 21.iv.12; individualCount: 1; sex: male; lifeStage: adult; recordedBy: Rafael, J.A. | Limeira-de-Oliveira, F. | Takiya, D.M. | et al.; identifiedBy: Allan Paulo Moreira dos Santos; institutionCode: DZRJ; basisOfRecord: PreservedSpecimen

Distribution: Brazil: PI, BA, MG, PR.

Notes: Species described in Souza et al. 2016b.

\section{Genus Flintiella Angrisano, 1995}

Notes: Genus firstly recorded from PI in Souza et al. 2016a.

\section{Flintiella harrisi Souza, Santos \& Takiya, 2016}

\section{Material}

a. country: Brazil; stateProvince: Piauí; municipality: Piracuruca; locality: Parque Nacional de Sete Cidades, Riacho da Piedade; maximumElevationInMeters: 169; verbatimCoordinates: $4^{\circ} 6^{\prime} 34$ "S, $41^{\circ} 43^{\prime} 39 " \mathrm{~W}$; samplingProtocol: Malaise intercept trap; verbatimEventDate: 21.iv.12; individualCount: 1; sex: male; lifeStage: adult; recordedBy: Rafael, J.A. | Limeira-de-Oliveira, F. | Takiya, D.M. | et al.; identifiedBy: Allan Paulo Moreira dos Santos; institutionCode: DZRJ; basisOfRecord: PreservedSpecimen 
Distribution: Brazil: PI.

Notes: Species described in Souza et al. 2016a.

\section{Genus Hydroptila Dalman, 1918}

Notes: Genus firstly recorded from PI in Souza et al. 2014a.

\section{Hydroptila florestani Souza, Santos \& Takiya, 2014}

\section{Materials}

a. country: Brazil; stateProvince: Piauí; municipality: Piracuruca; locality: Parque Nacional de Sete Cidades, Riacho da Piedade; maximumElevationInMeters: 169; verbatimCoordinates: $4^{\circ} 6^{\prime} 34$ "S, $41^{\circ} 43^{\prime} 39 " \mathrm{~W}$; samplingProtocol: Pennsylvania light trap; verbatimEventDate: 19.iv.12; individualCount: 2; sex: male; lifeStage: adult; recordedBy: Takiya, D.M.; identifiedBy: Allan Paulo Moreira dos Santos; institutionCode: DZRJ; basisOfRecord: PreservedSpecimen

b. $\quad$ country: Brazil; stateProvince: Piauí; municipality: Piracuruca; locality: Parque Nacional de Sete Cidades, Riacho da Piedade; maximumElevationInMeters: 169; verbatimCoordinates: $4^{\circ} 6^{\prime} 34$ "S, $41^{\circ} 43^{\prime} 39 " \mathrm{~W}$; samplingProtocol: Pennsylvania light trap; verbatimEventDate: 19.iv.12; individualCount: 2; sex: female; lifeStage: adult; recordedBy: Takiya, D.M.; identifiedBy: Allan Paulo Moreira dos Santos; institutionCode: DZRJ; basisOfRecord: PreservedSpecimen

c. country: Brazil; stateProvince: Piauí; municipality: Piracuruca; locality: Parque Nacional de Sete Cidades, Riacho da Piedade; maximumElevationInMeters: 169; verbatimCoordinates: $4^{\circ} 6{ }^{\prime} 34^{\prime \prime S}, 41^{\circ} 43^{\prime} 39 " \mathrm{~W}$; samplingProtocol: Malaise intercept trap; verbatimEventDate: 21.iv.12; individualCount: 3; sex: male; lifeStage: adult; recordedBy: Rafael, J.A. | Limeira-de-Oliveira, F. | Takiya, D.M. | et al.; identifiedBy: Allan Paulo Moreira dos Santos; institutionCode: DZRJ; basisOfRecord: PreservedSpecimen

d. country: Brazil; stateProvince: Piauí; municipality: Piracuruca; locality: Parque Nacional de Sete Cidades, Riacho da Piedade; maximumElevationInMeters: 169; verbatimCoordinates: $4^{\circ} 6^{\prime} 34$ "S, $41^{\circ} 43^{\prime} 39 " \mathrm{~W}$; samplingProtocol: Malaise intercept trap; verbatimEventDate: 21.iv.12; individualCount: 1; sex: female; lifeStage: adult; recordedBy: Rafael, J.A. | Limeira-de-Oliveira, F. | Takiya, D.M. | et al.; identifiedBy: Allan Paulo Moreira dos Santos; institutionCode: DZRJ; basisOfRecord: PreservedSpecimen

Distribution: Brazil: PI.

Notes: Species described in Souza et al. 2014a.

\section{Genus Neotrichia Morton, 1905}

Notes: New genus record for PI. 


\section{Neotrichia sp. 1}

\section{Materials}

a. country: Brazil; stateProvince: Piauí; municipality: Piracuruca; locality: Parque Nacional de Sete Cidades, Cachoeira do Riachão; maximumElevationInMeters: 171; verbatimCoordinates: $4^{\circ} 6^{\prime} 28$ "S, $41^{\circ} 40^{\prime} 13^{\prime \prime W}$; samplingProtocol: Pennsylvania light trap; verbatimEventDate: 19.iv.12; individualCount: 1; sex: male; lifeStage: adult; recordedBy: Takiya, D.M.; identifiedBy: Allan Paulo Moreira dos Santos; institutionCode: DZRJ; basisOfRecord: PreservedSpecimen

b. country: Brazil; stateProvince: Piauí; municipality: Piracuruca; locality: Parque Nacional de Sete Cidades, Riacho da Piedade; maximumElevationInMeters: 169;

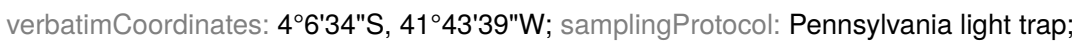
verbatimEventDate: 19.iv.12; individualCount: 16; sex: male; lifeStage: adult; recordedBy: Takiya, D.M.; identifiedBy: Allan Paulo Moreira dos Santos; institutionCode: DZRJ; basisOfRecord: PreservedSpecimen

c. country: Brazil; stateProvince: Piauí; municipality: Piracuruca; locality: Parque Nacional de Sete Cidades, Alojamento; maximumElevationInMeters: 193; verbatimCoordinates: $4^{\circ}$

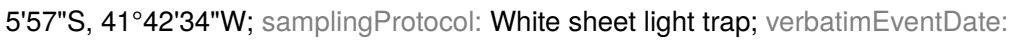
20.iv.12; individualCount: 1; sex: male; lifeStage: adult; recordedBy: Takiya, D.M.; identifiedBy: Allan Paulo Moreira dos Santos; institutionCode: DZRJ; basisOfRecord: PreservedSpecimen

d. country: Brazil; stateProvince: Piauí; municipality: Piracuruca; locality: Parque Nacional de Sete Cidades, Alojamento; maximumElevationInMeters: 193; verbatimCoordinates: $4^{\circ}$ 5'57"S, 4142'34"W; samplingProtocol: White sheet light trap; verbatimEventDate: 20.iv.12; individualCount: 1; sex: female; lifeStage: adult; recordedBy: Takiya, D.M.; identifiedBy: Allan Paulo Moreira dos Santos; institutionCode: DZRJ; basisOfRecord: PreservedSpecimen

\section{Neotrichia sp. 2*}

\section{Material}

a. country: Brazil; stateProvince: Piauí; municipality: Piracuruca; locality: Parque Nacional de Sete Cidades, Cachoeira do Riachão; maximumElevationInMeters: 171; verbatimCoordinates: $4^{\circ} 6^{\prime 2} 28^{\prime \prime S}, 41^{\circ} 40^{\prime} 13$ "W; samplingProtocol: Pennsylvania light trap; verbatimEventDate: 19.iv.12; individualCount: 1; sex: male; lifeStage: adult; recordedBy: Takiya, D.M.; identifiedBy: Allan Paulo Moreira dos Santos; institutionCode: DZRJ; basisOfRecord: PreservedSpecimen

\section{Neotrichia sp. 3}

\section{Material}

a. country: Brazil; stateProvince: Piauí; municipality: Piracuruca; locality: Parque Nacional de Sete Cidades, Cachoeira do Riachão; maximumElevationInMeters: 171; verbatimCoordinates: $4^{\circ} 6^{\prime} 28^{\prime \prime} \mathrm{S}, 41^{\circ} 40^{\prime} 13^{\prime \prime W}$; samplingProtocol: Pennsylvania light trap; verbatimEventDate: 19.iv.12; individualCount: 1; sex: male; lifeStage: adult; recordedBy: Takiya, D.M.; identifiedBy: Allan Paulo Moreira dos Santos; institutionCode: DZRJ; basisOfRecord: PreservedSpecimen 


\section{Neotrichia sp. 4}

\section{Materials}

a. country: Brazil; stateProvince: Piauí; municipality: Piracuruca; locality: Parque Nacional de Sete Cidades, Cachoeira do Riachão; maximumElevationInMeters: 171; verbatimCoordinates: $4^{\circ} 6^{\prime} 28^{\prime \prime} \mathrm{S}, 4^{\circ} 40^{\prime} 13$ "W; samplingProtocol: Pennsylvania light trap; verbatimEventDate: 19.iv.12; individualCount: 1; sex: male; lifeStage: adult; recordedBy: Takiya, D.M.; identifiedBy: Allan Paulo Moreira dos Santos; institutionCode: DZRJ; basisOfRecord: PreservedSpecimen

b. country: Brazil; stateProvince: Piauí; municipality: Piracuruca; locality: Parque Nacional de Sete Cidades, Riacho da Piedade; maximumElevationInMeters: 169; verbatimCoordinates: $4^{\circ} 6^{\prime} 34$ "S, $41^{\circ} 43^{\prime} 39 " \mathrm{~W}$; samplingProtocol: Pennsylvania light trap; verbatimEventDate: 19.iv.12; individualCount: 4; sex: male; lifeStage: adult; recordedBy: Takiya, D.M.; identifiedBy: Allan Paulo Moreira dos Santos; institutionCode: DZRJ; basisOfRecord: PreservedSpecimen

\section{Genus Ochrotrichia Mosely, 1934}

Notes: New genus record for PI.

\section{Ochrotrichia sp. 1}

\section{Material}

a. country: Brazil; stateProvince: Piauí; municipality: Piracuruca; locality: Parque Nacional de Sete Cidades, Cachoeira do Riachão; maximumElevationInMeters: 171; verbatimCoordinates: $4^{\circ} 6^{\prime} 28^{\prime \prime S}, 41^{\circ} 40^{\prime} 13 " \mathrm{~W}$; samplingProtocol: Pennsylvania light trap; verbatimEventDate: 19.iv.12; individualCount: 1; sex: male; lifeStage: adult; recordedBy: Takiya, D.M.; identifiedBy: Allan Paulo Moreira dos Santos; institutionCode: DZRJ; basisOfRecord: PreservedSpecimen

\section{Genus Oxyethira Eaton, 1873}

Notes: New genus record for PI.

\section{Oxyethira longissima Flint, 1974}

\section{Materials}

a. $\quad$ country: Brazil; stateProvince: Piauí; municipality: Piracuruca; locality: Parque Nacional de Sete Cidades, Cachoeira do Riachão; maximumElevationInMeters: 171; verbatimCoordinates: $4^{\circ} 6^{\prime} 28$ "S, $41^{\circ} 40^{\prime} 13^{\prime \prime} \mathrm{W}$; samplingProtocol: Pennsylvania light trap; verbatimEventDate: 18.iv.12; individualCount: 1; sex: male; lifeStage: adult; recordedBy: Takiya, D.M.; identifiedBy: Allan Paulo Moreira dos Santos; institutionCode: DZRJ; basisOfRecord: PreservedSpecimen

b. $\quad$ country: Brazil; stateProvince: Piauí; municipality: Piracuruca; locality: Parque Nacional de Sete Cidades, Cachoeira do Riachão; maximumElevationInMeters: 171; verbatimCoordinates: $4^{\circ} 6^{\prime} 28^{\prime \prime} \mathrm{S}, 41^{\circ} 40^{\prime} 13^{\prime \prime W}$; samplingProtocol: Pennsylvania light trap; verbatimEventDate: 19.iv.12; individualCount: 1; sex: male; lifeStage: adult; recordedBy: 
Takiya, D.M.; identifiedBy: Allan Paulo Moreira dos Santos; institutionCode: DZRJ; basisOfRecord: PreservedSpecimen

Distribution: Suriname. Brazil: AM, PI!.

Notes: New species record for Northeastern Brazil.

\section{Oxyethira merga Kelley, 1983}

\section{Material}

a. country: Brazil; stateProvince: Piauí; municipality: Piracuruca; locality: Parque Nacional de Sete Cidades, Cachoeira do Riachão; maximumElevationInMeters: 171; verbatimCoordinates: $4^{\circ} 6^{\prime} 28^{\prime \prime S}, 41^{\circ} 40^{\prime} 13$ "W; samplingProtocol: Pennsylvania light trap; verbatimEventDate: 18.iv.12; individualCount: 1; sex: male; lifeStage: adult; recordedBy: Takiya, D.M.; identifiedBy: Allan Paulo Moreira dos Santos; institutionCode: DZRJ; basisOfRecord: PreservedSpecimen

Distribution: Venezuela. Brazil: RR, PI!.

Notes: New species record for Northeastern Brazil.

\section{Oxyethira spissa Kelley, 1983}

\section{Material}

a. country: Brazil; stateProvince: Piauí; municipality: Piracuruca; locality: Parque Nacional de Sete Cidades, Cachoeira do Riachão; maximumElevationInMeters: 171; verbatimCoordinates: $4^{\circ} 6^{\prime} 28^{\prime \prime S}, 41^{\circ} 40^{\prime} 13^{\prime \prime W}$; samplingProtocol: Pennsylvania light trap; verbatimEventDate: 18.iv.12; individualCount: 1; sex: male; lifeStage: adult; recordedBy: Takiya, D.M.; identifiedBy: Wagner Rafael Maciel de Souza; institutionCode: DZRJ; basisOfRecord: PreservedSpecimen

Distribution: Brazil: PA, PI!.

Notes: New species record for Northeastern Brazil.

\section{Oxyethira tica* Harris \& Holzenthal, 1992}

\section{Materials}

a. country: Brazil; stateProvince: Piauí; municipality: Piracuruca; locality: Parque Nacional de Sete Cidades, Cachoeira do Riachão; maximumElevationInMeters: 171; verbatimCoordinates: $4^{\circ} 6^{\prime} 28^{\prime \prime} \mathrm{S}, 41^{\circ} 40^{\prime} 13^{\prime \prime W}$; samplingProtocol: Pennsylvania light trap; verbatimEventDate: 18.iv.12; individualCount: 1; sex: male; lifeStage: adult; recordedBy: Takiya, D.M.; identifiedBy: Allan Paulo Moreira dos Santos; institutionCode: DZRJ; basisOfRecord: PreservedSpecimen

b. country: Brazil; stateProvince: Piauí; municipality: Piracuruca; locality: Parque Nacional de Sete Cidades, Cachoeira do Riachão; maximumElevationInMeters: 171; verbatimCoordinates: $4^{\circ} 6^{\prime} 28$ "S, $41^{\circ} 40^{\prime} 13$ "W; samplingProtocol: Pennsylvania light trap; verbatimEventDate: 19.iv.12; individualCount: 1; sex: male; lifeStage: adult; recordedBy: 
Takiya, D.M.; identifiedBy: Allan Paulo Moreira dos Santos; institutionCode: DZRJ; basisOfRecord: PreservedSpecimen

c. country: Brazil; stateProvince: Piauí; municipality: Piracuruca; locality: Parque Nacional de Sete Cidades, Cachoeira do Riachão; maximumElevationInMeters: 171; verbatimCoordinates: $4^{\circ} 6^{\prime} 28^{\prime \prime S}, 41^{\circ} 40^{\prime} 13^{\prime \prime W}$; samplingProtocol: Pennsylvania light trap; verbatimEventDate: 19.iv.12; individualCount: 2; sex: male; lifeStage: adult; recordedBy: Takiya, D.M.; identifiedBy: Allan Paulo Moreira dos Santos; institutionCode: DZRJ; basisOfRecord: PreservedSpecimen

d. country: Brazil; stateProvince: Piauí; municipality: Piracuruca; locality: Parque Nacional de Sete Cidades, Cachoeira do Riachão; maximumElevationInMeters: 171; verbatimCoordinates: $4^{\circ} 6^{\prime} 28^{\prime \prime S}, 41^{\circ} 40^{\prime} 13 " \mathrm{~W}$; samplingProtocol: Pennsylvania light trap; verbatimEventDate: 20.iv.12; individualCount: 1; sex: male; lifeStage: adult; recordedBy: Takiya, D.M. | Rafael, J.A.; identifiedBy: Allan Paulo Moreira dos Santos; institutionCode: DZRJ; basisOfRecord: PreservedSpecimen

Distribution: Mexico. Honduras. Costa Rica. Panama. Guadeloupe. Dominica. Santa Lucia. Saint Vicent and the Grenadines. Granada. Trinidad and Tobago. Venezuela. Brazil: AM, PI!, CE!, MG, RJ. Ecuador.

Notes: New species record for Northeastern Brazil.

\section{Oxyethira sp. 1}

\section{Materials}

a. country: Brazil; stateProvince: Piauí; municipality: Piracuruca; locality: Parque Nacional de Sete Cidades, Cachoeira do Riachão; maximumElevationInMeters: 171; verbatimCoordinates: $4^{\circ} 6^{\prime} 28^{\prime \prime S}, 41^{\circ} 40^{\prime} 13 " \mathrm{~W}$; samplingProtocol: Pennsylvania light trap; verbatimEventDate: 18.iv.12; individualCount: 1; sex: male; lifeStage: adult; recordedBy: Takiya, D.M.; identifiedBy: Allan Paulo Moreira dos Santos; institutionCode: DZRJ; basisOfRecord: PreservedSpecimen

b. country: Brazil; stateProvince: Piauí; municipality: Piracuruca; locality: Parque Nacional de Sete Cidades, Riacho da Piedade; maximumElevationInMeters: 169; verbatimCoordinates: $4^{\circ} 6^{\prime} 34$ "S, $41^{\circ} 43^{\prime} 39$ "W; samplingProtocol: Pennsylvania light trap; verbatimEventDate: 19.iv.12; individualCount: 13; sex: male; lifeStage: adult; recordedBy: Takiya, D.M.; identifiedBy: Allan Paulo Moreira dos Santos; institutionCode: DZRJ; basisOfRecord: PreservedSpecimen

c. country: Brazil; stateProvince: Piauí; municipality: Piracuruca; locality: Parque Nacional de Sete Cidades, Alojamento; maximumElevationInMeters: 193; verbatimCoordinates: $4^{\circ}$ 5'57"S, 4142'34"W; samplingProtocol: White sheet light trap; verbatimEventDate: 20.iv.12; individualCount: 1; sex: male; lifeStage: adult; recordedBy: Takiya, D.M.; identifiedBy: Allan Paulo Moreira dos Santos; institutionCode: DZRJ; basisOfRecord: PreservedSpecimen

d. country: Brazil; stateProvince: Piauí; municipality: Piracuruca; locality: Parque Nacional de Sete Cidades, Alojamento; maximumElevationInMeters: 193; verbatimCoordinates: $4^{\circ}$ 5'57"S, 4142'34"W; samplingProtocol: White sheet light trap; verbatimEventDate: 20.iv.12; individualCount: 1; sex: female; lifeStage: adult; recordedBy: Takiya, D.M.; identifiedBy: Allan Paulo Moreira dos Santos; institutionCode: DZRJ; basisOfRecord: PreservedSpecimen 
Notes: Undescribed species.

\section{Oxyethira sp. 2}

\section{Materials}

a. country: Brazil; stateProvince: Piauí; municipality: Piracuruca; locality: Parque Nacional de Sete Cidades, Poço do Bananeira; maximumElevationInMeters: 158; verbatimCoordinates: $4^{\circ} 5^{\prime} 55.8^{\prime \prime S}, 41^{\circ} 40^{\prime} 33.8^{\prime \prime} \mathrm{W}$; samplingProtocol: Pennsylvania light trap; verbatimEventDate: 11.ii.13; individualCount: 3; sex: male; lifeStage: adult; recordedBy: Santos, A.P.M. | Takiya, D.M.; identifiedBy: Allan Paulo Moreira dos Santos; institutionCode: DZRJ; basisOfRecord: PreservedSpecimen

b. country: Brazil; stateProvince: Piauí; municipality: Piracuruca; locality: Parque Nacional de Sete Cidades, Poço do Bananeira; maximumElevationInMeters: 158; verbatimCoordinates: $4^{\circ} 5^{\prime} 55.8^{\prime \prime} \mathrm{S}, 41^{\circ} 40^{\prime} 33.8^{\prime \prime} \mathrm{W}$; sampling Protocol: Pennsylvania light trap; verbatimEventDate: 11.ii.13; individualCount: 1; sex: female; lifeStage: adult; recordedBy: Santos, A.P.M. | Takiya, D.M.; identifiedBy: Allan Paulo Moreira dos Santos; institutionCode: DZRJ; basisOfRecord: PreservedSpecimen

c. country: Brazil; stateProvince: Piauí; municipality: Piracuruca; locality: Parque Nacional de Sete Cidades, Riacho da Bananeira; maximumElevationInMeters: 189; verbatimCoordinates: $4^{\circ} 5^{\prime} 59$ "S, $41^{\circ} 40^{\prime} 48^{\prime \prime}$; sampling Protocol: Malaise intercept trap; verbatimEventDate: 19.iv.12; individualCount: 1; sex: male; lifeStage: adult; recordedBy: Rafael, J.A. | Limeira-de-Oliveira, F. | Takiya, D.M. | et al.; identifiedBy: Allan Paulo Moreira dos Santos; institutionCode: DZRJ; basisOfRecord: PreservedSpecimen

d. country: Brazil; stateProvince: Piauí; municipality: Piracuruca; locality: Parque Nacional de Sete Cidades, Cachoeira do Riachão; maximumElevationInMeters: 171; verbatimCoordinates: $4^{\circ} 6^{\prime} 28^{\prime \prime S}, 41^{\circ} 40^{\prime} 13$ "W; samplingProtocol: Pennsylvania light trap; verbatimEventDate: 19.iv.12; individualCount: 4; sex: male; lifeStage: adult; recordedBy: Takiya, D.M.; identifiedBy: Allan Paulo Moreira dos Santos; institutionCode: DZRJ; basisOfRecord: PreservedSpecimen

e. country: Brazil; stateProvince: Piauí; municipality: Piracuruca; locality: Parque Nacional de Sete Cidades, Riacho da Piedade; maximumElevationInMeters: 169; verbatimCoordinates: $4^{\circ} 6^{\prime} 34$ "S, $41^{\circ} 43^{\prime} 39$ "W; samplingProtocol: Pennsylvania light trap; verbatimEventDate: 19.iv.12; individualCount: 2; sex: male; lifeStage: adult; recordedBy: Takiya, D.M.; identifiedBy: Allan Paulo Moreira dos Santos; institutionCode: DZRJ; basisOfRecord: PreservedSpecimen

Notes: Undescribed species.

\section{Oxyethira sp. 3}

\section{Material}

a. country: Brazil; stateProvince: Piauí; municipality: Piracuruca; locality: Parque Nacional de Sete Cidades, Cachoeira do Riachão; maximumElevationInMeters: 171; verbatimCoordinates: $4^{\circ} 6^{\prime} 28^{\prime \prime S}, 41^{\circ} 40^{\prime} 13 " \mathrm{~W}$; samplingProtocol: Pennsylvania light trap; verbatimEventDate: 19.iv.12; individualCount: 1; sex: male; lifeStage: adult; recordedBy: Takiya, D.M.; identifiedBy: Allan Paulo Moreira dos Santos; institutionCode: DZRJ; basisOfRecord: PreservedSpecimen 
Notes: Undescribed species.

\section{Oxyethira sp. 4}

\section{Material}

a. country: Brazil; stateProvince: Piauí; municipality: Piracuruca; locality: Parque Nacional de Sete Cidades, Poço do Bananeira; maximumElevationInMeters: 158; verbatimCoordinates: $4^{\circ} 5^{\prime} 55.8^{\prime \prime S}, 41^{\circ} 40^{\prime} 33.8^{\prime \prime} \mathrm{W}$; samplingProtocol: Pennsylvania light trap; verbatimEventDate: 9.ii.13; individualCount: 1; sex: male; lifeStage: adult; recordedBy: Santos, A.P.M. | Takiya, D.M.; identifiedBy: Allan Paulo Moreira dos Santos; institutionCode: DZRJ; basisOfRecord: PreservedSpecimen

Notes: Undescribed species.

\section{Oxyethira sp. 5}

\section{Materials}

a. country: Brazil; stateProvince: Piauí; municipality: Piracuruca; locality: Parque Nacional de Sete Cidades, Cachoeira do Riachão; maximumElevationInMeters: 171; verbatimCoordinates: $4^{\circ} 6^{\prime 2} 28^{\prime \prime S}, 41^{\circ} 40^{\prime} 13$ "W; samplingProtocol: Pennsylvania light trap; verbatimEventDate: 19.iv.12; individualCount: 3; sex: male; lifeStage: adult; recordedBy: Takiya, D.M.; identifiedBy: Wagner Rafael Maciel de Souza; institutionCode: DZRJ; basisOfRecord: PreservedSpecimen

b. country: Brazil; stateProvince: Piauí; municipality: Piracuruca; locality: Parque Nacional de Sete Cidades, Riacho da Piedade; maximumElevationInMeters: 169;

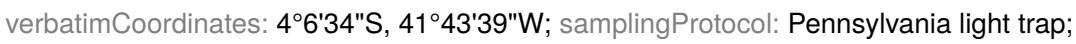
verbatimEventDate: 19.iv.12; individualCount: 1; sex: male; lifeStage: adult; recordedBy: Takiya, D.M.; identifiedBy: Allan Paulo Moreira dos Santos; institutionCode: DZRJ; basisOfRecord: PreservedSpecimen

Notes: Undescribed species.

\section{Family Leptoceridae}

\section{Genus Nectopsyche Müller, 1879}

\section{Nectopsyche cf. multilineata Flint, 1983}

\section{Material}

a. country: Brazil; stateProvince: Piauí; municipality: Piracuruca; locality: Parque Nacional de Sete Cidades, Centro de visitantes; maximumElevationInMeters: 202; verbatimCoordinates: $4^{\circ} 6^{\prime} 20^{\prime} \mathrm{S}, 41^{\circ} 41^{\prime} 52 \mathrm{~W} \mathrm{~W}$; sampling Protocol: White sheet light trap; verbatimEventDate: 19.iv.12; individualCount: 2; sex: female; lifeStage: adult; recordedBy: Takiya, D.M.; identifiedBy: Ana Lucia Henriques Oliveira; institutionCode: DZRJ; basisOfRecord: PreservedSpecimen 
Distribution: Venezuela. Brazil: RR, PI!. Argentina.

Notes: if confirmed, new species record for Northeastern Brazil.

\section{Nectopsyche muhni Flint, 1983}

\section{Materials}

a. country: Brazil; stateProvince: Piauí; municipality: Piracuruca; locality: Parque Nacional de Sete Cidades, Cachoeira do Riachão; maximumElevationInMeters: 171; verbatimCoordinates: $4^{\circ} 6^{\prime} 28$ "S, $41^{\circ} 40^{\prime} 13$ "W; samplingProtocol: Pennsylvania light trap; verbatimEventDate: 18.iv.12; individualCount: 1; sex: male; lifeStage: adult; recordedBy: Takiya, D.M.; identifiedBy: Ana Lucia Henriques Oliveira; institutionCode: DZRJ; basisOfRecord: PreservedSpecimen

b. $\quad$ country: Brazil; stateProvince: Piauí; municipality: Piracuruca; locality: Parque Nacional de Sete Cidades, Cachoeira do Riachão; maximumElevationInMeters: 171; verbatimCoordinates: $4^{\circ} 6^{\prime} 28$ "S, $41^{\circ} 40^{\prime} 13$ "W; samplingProtocol: Pennsylvania light trap; verbatimEventDate: 18.iv.12; individualCount: 7; sex: male; lifeStage: adult; recordedBy: Takiya, D.M.; identifiedBy: Allan Paulo Moreira dos Santos; institutionCode: DZRJ; basisOfRecord: PreservedSpecimen

c. country: Brazil; stateProvince: Piauí; municipality: Piracuruca; locality: Parque Nacional de Sete Cidades, Cachoeira do Riachão; maximumElevationInMeters: 171; verbatimCoordinates: $4^{\circ} 6^{\prime} 28^{\prime \prime} \mathrm{S}, 41^{\circ} 40^{\prime} 13^{\prime \prime W}$; samplingProtocol: Pennsylvania light trap; verbatimEventDate: 18.iv.12; individualCount: 8; sex: male; lifeStage: adult; recordedBy: Takiya, D.M.; identifiedBy: Ana Lucia Henriques Oliveira; institutionCode: DZRJ; basisOfRecord: PreservedSpecimen

d. country: Brazil; stateProvince: Piauí; municipality: Piracuruca; locality: Parque Nacional de Sete Cidades, Cachoeira do Riachão; maximumElevationInMeters: 171; verbatimCoordinates: $4^{\circ} 6^{\prime} 28$ "S, $41^{\circ} 40^{\prime} 13$ "W; samplingProtocol: Pennsylvania light trap; verbatimEventDate: 18.iv.12; individualCount: 3; sex: male; lifeStage: adult; recordedBy: Takiya, D.M.; identifiedBy: Ana Lucia Henriques Oliveira; institutionCode: DZRJ; basisOfRecord: PreservedSpecimen

e. country: Brazil; stateProvince: Piauí; municipality: Piracuruca; locality: Parque Nacional de Sete Cidades, Cachoeira do Riachão; maximumElevationInMeters: 171; verbatimCoordinates: $4^{\circ} 6^{\prime} 28^{\prime \prime S}, 41^{\circ} 40^{\prime} 13^{\prime \prime W}$; samplingProtocol: Pennsylvania light trap; verbatimEventDate: 18.iv.12; individualCount: 8; sex: male; lifeStage: adult; recordedBy: Takiya, D.M.; identifiedBy: Ana Lucia Henriques Oliveira; institutionCode: DZRJ; basisOfRecord: PreservedSpecimen

f. country: Brazil; stateProvince: Piauí; municipality: Piracuruca; locality: Parque Nacional de Sete Cidades, Riacho da Piedade; maximumElevationInMeters: 169; verbatimCoordinates: $4^{\circ} 6^{\prime} 34$ "S, $41^{\circ} 43^{\prime} 39$ "W; samplingProtocol: Pennsylvania light trap; verbatimEventDate: 19.iv.12; individualCount: 1; sex: male; lifeStage: adult; recordedBy: Takiya, D.M.; identifiedBy: Ana Lucia Henriques Oliveira; institutionCode: DZRJ; basisOfRecord: PreservedSpecimen

Distribution: Venezuela. Guyana. Suriname. Brazil: RR, PI!, MG, SP, RJ. Ecuador. Peru. Bolivia. Paraguay. Argentina.

Notes: New species record for Northeastern Brazil. 


\section{Nectopsyche sp.}

\section{Materials}

a. country: Brazil; stateProvince: Piauí; municipality: Piracuruca; locality: Parque Nacional de Sete Cidades, Alojamento; maximumElevationInMeters: 193; verbatimCoordinates: $4^{\circ}$ 5'57"S, 4142'34"W; samplingProtocol: White sheet light trap; verbatimEventDate: 20.iv.12; individualCount: 1; sex: male; lifeStage: adult; recordedBy: Takiya, D.M.; identifiedBy: Allan Paulo Moreira dos Santos; institutionCode: DZRJ; basisOfRecord: PreservedSpecimen

b. country: Brazil; stateProvince: Piauí; municipality: Piracuruca; locality: Parque Nacional de Sete Cidades, Alojamento; maximumElevationInMeters: 193; verbatimCoordinates: $4^{\circ}$ 5'57"S, 4142'34"W; samplingProtocol: White sheet light trap; verbatimEventDate: 20.iv.12; individualCount: 1; sex: male; lifeStage: adult; recordedBy: Takiya, D.M.; identifiedBy: Ana Lucia Henriques Oliveira; institutionCode: DZRJ; basisOfRecord: PreservedSpecimen

Notes: Undescribed species.

\section{Genus Oecetis McLachlan, 1877}

\section{Oecetis sp. 1}

\section{Material}

a. country: Brazil; stateProvince: Piauí; municipality: Piracuruca; locality: Parque Nacional de Sete Cidades, Cachoeira do Riachão; maximumElevationInMeters: 171; verbatimCoordinates: $4^{\circ} 6^{\prime} 28^{\prime \prime} \mathrm{S}, 41^{\circ} 40^{\prime} 13^{\prime \prime} \mathrm{W}$; samplingProtocol: Pennsylvania light trap; verbatimEventDate: 18.iv.12; individualCount: 1; sex: male; lifeStage: adult; recordedBy: Takiya, D.M.; identifiedBy: Ana Lucia Henriques Oliveira; institutionCode: DZRJ; basisOfRecord: PreservedSpecimen

Notes: Undescribed species.

\section{Oecetis connata Flint, 1974}

\section{Materials}

a. country: Brazil; stateProvince: Piauí; municipality: Piracuruca; locality: Parque Nacional de Sete Cidades, Riacho da Bananeira; maximumElevationInMeters: 189; verbatimCoordinates: $4^{\circ} 5^{\prime} 59^{\prime \prime S}, 41^{\circ} 40^{\prime} 48 \mathrm{WW}$; samplingProtocol: Pennsylvania light trap; verbatimEventDate: 18.iv.12; individualCount: 4; sex: male; lifeStage: adult; recordedBy: Takiya, D.M.; identifiedBy: Ana Lucia Henriques Oliveira; institutionCode: DZRJ; basisOfRecord: PreservedSpecimen

b. $\quad$ country: Brazil; stateProvince: Piauí; municipality: Piracuruca; locality: Parque Nacional de Sete Cidades, Riacho da Bananeira; maximumElevationInMeters: 189; verbatim Coordinates: $4^{\circ} 5^{\prime} 59^{\prime \prime S}, 41^{\circ} 40^{\prime} 48 \mathrm{WW}$; samplingProtocol: Pennsylvania light trap; verbatimEventDate: 18.iv.12; individualCount: 1; sex: female; lifeStage: adult; recordedBy: Takiya, D.M.; identifiedBy: Ana Lucia Henriques Oliveira; institutionCode: DZRJ; basisOfRecord: PreservedSpecimen 
c. country: Brazil; stateProvince: Piauí; municipality: Piracuruca; locality: Parque Nacional de Sete Cidades, Riacho da Bananeira; maximumElevationInMeters: 189; verbatimCoordinates: $4^{\circ} 5^{\prime} 59$ "S, $41^{\circ} 40^{\prime} 48$ "W; samplingProtocol: Malaise intercept trap; verbatimEventDate: 18.iv.12; individualCount: 2; sex: male; lifeStage: adult; recordedBy: Rafael, J.A. | Limeira-de-Oliveira, F. | Takiya, D.M. | et al.; identifiedBy: Ana Lucia Henriques Oliveira; institutionCode: DZRJ; basisOfRecord: PreservedSpecimen

d. country: Brazil; stateProvince: Piauí; municipality: Piracuruca; locality: Parque Nacional de Sete Cidades, Cachoeira do Riachão; maximumElevationInMeters: 171; verbatimCoordinates: $4^{\circ} 6^{\prime} 28$ "S, $41^{\circ} 40^{\prime} 13^{\prime \prime} \mathrm{W}$; samplingProtocol: Pennsylvania light trap; verbatimEventDate: 18.iv.12; individualCount: 3; sex: male; lifeStage: adult; recordedBy: Takiya, D.M.; identifiedBy: Ana Lucia Henriques Oliveira; institutionCode: DZRJ; basisOfRecord: PreservedSpecimen

e. $\quad$ country: Brazil; stateProvince: Piauí; municipality: Piracuruca; locality: Parque Nacional de Sete Cidades, Cachoeira do Riachão; maximumElevationInMeters: 171; verbatimCoordinates: $4^{\circ} 6^{\prime} 28$ "S, $41^{\circ} 40^{\prime} 13$ "W; samplingProtocol: Pennsylvania light trap; verbatimEventDate: 18.iv.12; individualCount: 5; sex: female; lifeStage: adult; recordedBy: Takiya, D.M.; identifiedBy: Ana Lucia Henriques Oliveira; institutionCode: DZRJ; basisOfRecord: PreservedSpecimen

f. country: Brazil; stateProvince: Piauí; municipality: Piracuruca; locality: Parque Nacional de Sete Cidades, Cachoeira do Riachão; maximumElevationInMeters: 171; verbatimCoordinates: $4^{\circ} 6^{\prime} 28^{\prime \prime} \mathrm{S}, 41^{\circ} 40^{\prime} 13^{\prime \prime} \mathrm{W}$; samplingProtocol: Pennsylvania light trap; verbatimEventDate: 18.iv.12; individualCount: 1; sex: male; lifeStage: adult; recordedBy: Takiya, D.M.; identifiedBy: Ana Lucia Henriques Oliveira; institutionCode: DZRJ; basisOfRecord: PreservedSpecimen

g. country: Brazil; stateProvince: Piauí; municipality: Piracuruca; locality: Parque Nacional de Sete Cidades, Cachoeira do Riachão; maximumElevationInMeters: 171; verbatimCoordinates: $4^{\circ} 6^{\prime} 28^{\prime \prime S}, 41^{\circ} 40^{\prime} 13^{\prime \prime W}$; samplingProtocol: Pennsylvania light trap; verbatimEventDate: 18.iv.12; individualCount: 3; sex: female; lifeStage: adult; recordedBy: Takiya, D.M.; identifiedBy: Ana Lucia Henriques Oliveira; institutionCode: DZRJ; basisOfRecord: PreservedSpecimen

h. country: Brazil; stateProvince: Piauí; municipality: Piracuruca; locality: Parque Nacional de Sete Cidades, Alojamento; maximumElevationInMeters: 193; verbatimCoordinates: $4^{\circ}$ 5'57"S, 4142'34"W; samplingProtocol: White sheet light trap; verbatimEventDate: 18.iv.12; individualCount: 1; sex: female; lifeStage: adult; recordedBy: Takiya, D.M.; identifiedBy: Ana Lucia Henriques Oliveira; institutionCode: DZRJ; basisOfRecord: PreservedSpecimen

i. country: Brazil; stateProvince: Piauí; municipality: Piracuruca; locality: Parque Nacional de Sete Cidades, Riacho da Piedade; maximumElevationInMeters: 169; verbatimCoordinates: $4^{\circ} 6^{\prime} 34$ "S, $41^{\circ} 43^{\prime} 39 " \mathrm{~W}$; samplingProtocol: Malaise intercept trap; verbatimEventDate: 18.iv.12; individualCount: 1; sex: male; lifeStage: adult; recordedBy: Rafael, J.A. | Limeira-de-Oliveira, F. | Takiya, D.M. | et al.; identifiedBy: Ana Lucia Henriques Oliveira; institutionCode: DZRJ; basisOfRecord: PreservedSpecimen

j. country: Brazil; stateProvince: Piauí; municipality: Piracuruca; locality: Parque Nacional de Sete Cidades, Riacho da Piedade; maximumElevationInMeters: 169; verbatimCoordinates: $4^{\circ} 6^{\prime} 34$ "S, 41 ${ }^{\circ} 43^{\prime} 39$ "W; samplingProtocol: Malaise intercept trap; verbatimEventDate: 18.iv.12; individualCount: 1; sex: female; lifeStage: adult; recordedBy: Rafael, J.A. | Limeira-de-Oliveira, F. | Takiya, D.M. | et al.; identifiedBy: Ana Lucia Henriques Oliveira; institutionCode: DZRJ; basisOfRecord: PreservedSpecimen

k. country: Brazil; stateProvince: Piauí; municipality: Piracuruca; locality: Parque Nacional de Sete Cidades, Riacho da Bananeira; maximumElevationInMeters: 189; 
verbatimCoordinates: $4^{\circ} 5^{\prime} 59^{\prime \prime} \mathrm{S}, 41^{\circ} 40^{\prime} 48$ "W; samplingProtocol: Malaise intercept trap; verbatimEventDate: 19.iv.12; individualCount: 1; sex: male; lifeStage: adult; recordedBy: Rafael, J.A. | Limeira-de-Oliveira, F. | Takiya, D.M. | et al.; identifiedBy: Ana Lucia Henriques Oliveira; institutionCode: DZRJ; basisOfRecord: PreservedSpecimen

I. country: Brazil; stateProvince: Piauí; municipality: Piracuruca; locality: Parque Nacional de Sete Cidades, Riacho da Bananeira; maximumElevationInMeters: 189; verbatimCoordinates: $4^{\circ} 5^{\prime} 59$ "S, $41^{\circ} 40^{\prime} 48^{\prime \prime}$; sampling Protocol: Malaise intercept trap; verbatimEventDate: 19.iv.12; individualCount: 1; sex: female; lifeStage: adult; recordedBy: Rafael, J.A. | Limeira-de-Oliveira, F. | Takiya, D.M. | et al.; identifiedBy: Ana Lucia Henriques Oliveira; institutionCode: DZRJ; basisOfRecord: PreservedSpecimen

m. country: Brazil; stateProvince: Piauí; municipality: Piracuruca; locality: Parque Nacional de Sete Cidades, Riacho da Bananeira; maximumElevationInMeters: 189; verbatimCoordinates: $4^{\circ} 5^{\prime} 59^{\prime \prime S}, 41^{\circ} 40^{\prime} 48 \mathrm{WW}$; samplingProtocol: Malaise intercept trap; verbatimEventDate: 19.iv.12; individualCount: 2; sex: male; lifeStage: adult; recordedBy: Rafael, J.A. | Limeira-de-Oliveira, F. | Takiya, D.M. | et al.; identifiedBy: Ana Lucia Henriques Oliveira; institutionCode: DZRJ; basisOfRecord: PreservedSpecimen

n. country: Brazil; stateProvince: Piauí; municipality: Piracuruca; locality: Parque Nacional de Sete Cidades, Riacho da Piedade; maximumElevationInMeters: 169; verbatim Coordinates: $4^{\circ} 6^{\prime} 34^{\prime \prime S}, 41^{\circ} 43^{\prime} 39 " \mathrm{~W}$; samplingProtocol: Pennsylvania light trap; verbatimEventDate: 19.iv.12; individualCount: 4; sex: male; lifeStage: adult; recordedBy: Takiya, D.M.; identifiedBy: Ana Lucia Henriques Oliveira; institutionCode: DZRJ; basisOfRecord: PreservedSpecimen

o. country: Brazil; stateProvince: Piauí; municipality: Piracuruca; locality: Parque Nacional de Sete Cidades, Riacho da Piedade; maximumElevationInMeters: 169; verbatimCoordinates: $4^{\circ} 6^{\prime} 34$ "S, 4143'39"W; samplingProtocol: Pennsylvania light trap; verbatimEventDate: 19.iv.12; individualCount: 1; sex: female; lifeStage: adult; recordedBy: Takiya, D.M.; identifiedBy: Ana Lucia Henriques Oliveira; institutionCode: DZRJ; basisOfRecord: PreservedSpecimen

p. country: Brazil; stateProvince: Piauí; municipality: Piracuruca; locality: Parque Nacional de Sete Cidades, Alojamento; maximumElevationInMeters: 193; verbatimCoordinates: $4^{\circ}$ 5'57"S, 4142'34"W; samplingProtocol: White sheet light trap; verbatimEventDate: 20.iv.12; individualCount: 38; sex: male; lifeStage: adult; recordedBy: Takiya, D.M.; identifiedBy: Ana Lucia Henriques Oliveira; institutionCode: DZRJ; basisOfRecord: PreservedSpecimen

q. country: Brazil; stateProvince: Piauí; municipality: Piracuruca; locality: Parque Nacional de Sete Cidades, Alojamento; maximumElevationInMeters: 193; verbatimCoordinates: $4^{\circ}$ 5'57"S, 4142'34"W; samplingProtocol: White sheet light trap; verbatimEventDate: 20.iv.12; individualCount: 15; sex: female; lifeStage: adult; recordedBy: Takiya, D.M.; identifiedBy: Ana Lucia Henriques Oliveira; institutionCode: DZRJ; basisOfRecord: PreservedSpecimen

r. country: Brazil; stateProvince: Piauí; municipality: Piracuruca; locality: Parque Nacional de Sete Cidades, Riacho da Piedade; maximumElevationInMeters: 169; verbatimCoordinates: $4^{\circ} 6^{\prime} 34$ "S, 41 ${ }^{\circ} 43^{\prime} 39$ "W; samplingProtocol: Malaise intercept trap; verbatimEventDate: 21.iv.12; individualCount: 2; sex: male; lifeStage: adult; recordedBy: Rafael, J.A. | Limeira-de-Oliveira, F. | Takiya, D.M. | et al.; identifiedBy: Ana Lucia Henriques Oliveira; institutionCode: DZRJ; basisOfRecord: PreservedSpecimen

s. $\quad$ country: Brazil; stateProvince: Piauí; municipality: Piracuruca; locality: Parque Nacional de Sete Cidades, Riacho da Piedade; maximumElevationInMeters: 169; verbatimCoordinates: $4^{\circ} 6^{\prime} 34$ "S, $41^{\circ} 43^{\prime} 39^{\prime \prime W}$; sampling Protocol: Malaise intercept trap; verbatimEventDate: 21.iv.12; individualCount: 4; sex: female; lifeStage: adult; recordedBy: 
Rafael, J.A. | Limeira-de-Oliveira, F. | Takiya, D.M. | et al.; identifiedBy: Ana Lucia Henriques Oliveira; institutionCode: DZRJ; basisOfRecord: PreservedSpecimen

Distribution: Guyana. Suriname. Brazil: PA, AM, PI, MT, BA.

Notes: Species firstly recorded from PI in Henriques-Oliveira et al. 2014.

\section{Family Philopotamidae}

Notes: New family record for PI.

\section{Genus Chimarra Stephens, 1829}

Notes: New genus record for PI.

\section{Chimarra (Chimarra) sp. 1}

\section{Materials}

a. country: Brazil; stateProvince: Piauí; municipality: Piracuruca; locality: Parque Nacional de Sete Cidades, Cachoeira do Riachão; maximumElevationInMeters: 171; verbatimCoordinates: $4^{\circ} 6^{\prime} 28^{\prime \prime S}, 41^{\circ} 40^{\prime} 13 " \mathrm{~W}$; samplingProtocol: Pennsylvania light trap; verbatimEventDate: 18.iv.12; individualCount: 2; sex: male; lifeStage: adult; recordedBy: Takiya, D.M.; identifiedBy: Allan Paulo Moreira dos Santos; institutionCode: DZRJ; basisOfRecord: PreservedSpecimen

b. country: Brazil; stateProvince: Piauí; municipality: Piracuruca; locality: Parque Nacional de Sete Cidades, Cachoeira do Riachão; maximumElevationInMeters: 171; verbatimCoordinates: $4^{\circ} 6^{\prime} 28^{\prime \prime S}, 41^{\circ} 40^{\prime} 13 " \mathrm{~W}$; samplingProtocol: Malaise intercept trap; verbatimEventDate: 18.iv.12; individualCount: 1; sex: male; lifeStage: adult; recordedBy: Rafael, J.A. | Limeira-de-Oliveira, F. | Takiya, D.M. | et al.; identifiedBy: Allan Paulo Moreira dos Santos; institutionCode: DZRJ; basisOfRecord: PreservedSpecimen

Notes: Undescribed species.

\section{Chimarra (Chimarra) sp. 2}

\section{Materials}

a. country: Brazil; stateProvince: Piauí; municipality: Piracuruca; locality: Parque Nacional de Sete Cidades, Riacho da Piedade; maximumElevationInMeters: 169; verbatimCoordinates: $4^{\circ} 6^{\prime} 34$ "S, $41^{\circ} 43^{\prime} 39$ "W; samplingProtocol: Malaise intercept trap; verbatimEventDate: 18.iv.12; individualCount: 1; sex: male; lifeStage: adult; recordedBy: Rafael, J.A. | Limeira-de-Oliveira, F. | Takiya, D.M. | et al.; identifiedBy: Allan Paulo Moreira dos Santos; institutionCode: DZRJ; basisOfRecord: PreservedSpecimen

b. $\quad$ country: Brazil; stateProvince: Piauí; municipality: Piracuruca; locality: Parque Nacional de Sete Cidades, Cachoeira do Riachão; maximumElevationInMeters: 171; verbatimCoordinates: $4^{\circ} 6^{\prime} 28^{\prime \prime S}, 41^{\circ} 40^{\prime} 13 " \mathrm{~W}$; samplingProtocol: Pennsylvania light trap; verbatimEventDate: 19.iv.12; individualCount: 1; sex: male; lifeStage: adult; recordedBy: Takiya, D.M.; identifiedBy: Allan Paulo Moreira dos Santos; institutionCode: DZRJ; basisOfRecord: PreservedSpecimen 
Notes: Undescribed species.

\section{Chimarra (Chimarra) sp. 3}

\section{Materials}

a. country: Brazil; stateProvince: Piauí; municipality: Piracuruca; locality: Parque Nacional de Sete Cidades, Olho d'água Piscina do Bacuri; maximumElevationInMeters: 171; verbatimCoordinates: $4^{\circ} 6^{\prime} 1.2^{\prime \prime} \mathrm{S}, 41^{\circ} 42^{\prime} 38.8^{\prime \prime} \mathrm{W}$; samplingProtocol: White sheet light trap; verbatimEventDate: 18.iv.12; individualCount: 1; sex: male; lifeStage: adult; recordedBy: Takiya, D.M.; identifiedBy: Allan Paulo Moreira dos Santos; institutionCode: DZRJ; basisOfRecord: PreservedSpecimen

b. country: Brazil; stateProvince: Piauí; municipality: Piracuruca; locality: Parque Nacional de Sete Cidades, Olho d'água Piscina do Bacuri; maximumElevationInMeters: 171; verbatimCoordinates: $4^{\circ} 6^{\prime} 1.2^{\prime \prime} \mathrm{S}, 4^{\circ} 42^{\prime} 38.8 \mathrm{~W}$; samplingProtocol: White sheet light trap; verbatimEventDate: 18.iv.12; individualCount: 1; sex: female; lifeStage: adult; recordedBy: Takiya, D.M.; identifiedBy: Allan Paulo Moreira dos Santos; institutionCode: DZRJ; basisOfRecord: PreservedSpecimen

c. country: Brazil; stateProvince: Piauí; municipality: Piracuruca; locality: Parque Nacional de Sete Cidades, Cachoeira do Riachão; maximumElevationInMeters: 171; verbatimCoordinates: $4^{\circ} 6^{\prime 2} 28^{\prime \prime S}, 41^{\circ} 40^{\prime} 13$ "W; samplingProtocol: Pennsylvania light trap; verbatimEventDate: 19.iv.12; individualCount: 2; sex: male; lifeStage: adult; recordedBy: Takiya, D.M.; identifiedBy: Allan Paulo Moreira dos Santos; institutionCode: DZRJ; basisOfRecord: PreservedSpecimen

d. country: Brazil; stateProvince: Piauí; municipality: Piracuruca; locality: Parque Nacional de Sete Cidades, Centro de visitantes; maximumElevationInMeters: 202; verbatimCoordinates: $4^{\circ} 6^{\prime} 20$ "S, $41^{\circ} 41^{\prime} 52$ "W; samplingProtocol: White sheet light trap; verbatimEventDate: 19.iv.12; individualCount: 2; sex: male; lifeStage: adult; recordedBy: Takiya, D.M.; identifiedBy: Allan Paulo Moreira dos Santos; institutionCode: DZRJ; basisOfRecord: PreservedSpecimen

Notes: Undescribed species.

\section{Chimarra (Curgia) jugescens Flint, 1998}

\section{Materials}

a. country: Brazil; stateProvince: Piauí; municipality: Piracuruca; locality: Parque Nacional de Sete Cidades, Riacho da Bananeira; maximumElevationInMeters: 189; verbatimCoordinates: $4^{\circ} 5^{\prime} 59$ "S, $41^{\circ} 40^{\prime} 48 \mathrm{WW}$; samplingProtocol: Malaise intercept trap; verbatimEventDate: 18.iv.12; individualCount: 1; sex: male; lifeStage: adult; recordedBy: Rafael, J.A. | Limeira-de-Oliveira, F. | Takiya, D.M. | et al.; identifiedBy: Allan Paulo Moreira dos Santos; institutionCode: DZRJ; basisOfRecord: PreservedSpecimen

b. country: Brazil; stateProvince: Piauí; municipality: Piracuruca; locality: Parque Nacional de Sete Cidades, Cachoeira do Riachão; maximumElevationInMeters: 171; verbatimCoordinates: $4^{\circ} 6^{\prime} 28^{\prime \prime S}, 41^{\circ} 40^{\prime} 13 " \mathrm{~W}$; samplingProtocol: Malaise intercept trap; verbatimEventDate: 18.iv.12; individualCount: 2; sex: male; lifeStage: adult; recordedBy: Rafael, J.A. | Limeira-de-Oliveira, F. | Takiya, D.M. | et al.; identifiedBy: Allan Paulo Moreira dos Santos; institutionCode: DZRJ; basisOfRecord: PreservedSpecimen 
c. country: Brazil; stateProvince: Piauí; municipality: Piracuruca; locality: Parque Nacional de Sete Cidades, Riacho da Bananeira; maximumElevationInMeters: 189;

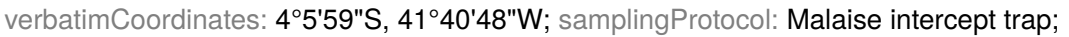
verbatimEventDate: 19.iv.12; individualCount: 3; sex: male; lifeStage: adult; recordedBy: Rafael, J.A. | Limeira-de-Oliveira, F. | Takiya, D.M. | et al.; identifiedBy: Allan Paulo Moreira dos Santos; institutionCode: DZRJ; basisOfRecord: PreservedSpecimen

d. country: Brazil; stateProvince: Piauí; municipality: Piracuruca; locality: Parque Nacional de Sete Cidades, Riacho da Bananeira; maximumElevationInMeters: 189; verbatimCoordinates: $4^{\circ} 5^{\prime} 59$ "S, $41^{\circ} 40^{\prime} 48^{\prime \prime W}$; samplingProtocol: Malaise intercept trap; verbatimEventDate: 19.iv.12; individualCount: 3; sex: female; lifeStage: adult; recordedBy: Rafael, J.A. | Limeira-de-Oliveira, F. | Takiya, D.M. | et al.; identifiedBy: Allan Paulo Moreira dos Santos; institutionCode: DZRJ; basisOfRecord: PreservedSpecimen

Distribution: Brazil: PA, AM, PI!.

Notes: New species record for Northeastern Brazil.

\section{Family Polycentropodidae}

Notes: New family record for PI.

\section{Genus Cernotina Ross, 1938}

Notes: New genus record for PI.

\section{Cernotina sp. 1}

\section{Materials}

a. country: Brazil; stateProvince: Piauí; municipality: Piracuruca; locality: Parque Nacional de Sete Cidades, Riacho da Piedade; maximumElevationInMeters: 169; verbatimCoordinates: $4^{\circ} 6^{\prime} 34$ "S, $41^{\circ} 43^{\prime} 39$ "W; sampling Protocol: Malaise intercept trap; verbatimEventDate: 18.iv.12; individualCount: 1; sex: male; lifeStage: adult; recordedBy: Rafael, J.A. | Limeira-de-Oliveira, F. | Takiya, D.M. | et al.; identifiedBy: Allan Paulo Moreira dos Santos; institutionCode: DZRJ; basisOfRecord: PreservedSpecimen

b. country: Brazil; stateProvince: Piauí; municipality: Piracuruca; locality: Parque Nacional de Sete Cidades, Riacho da Piedade; maximumElevationInMeters: 169; verbatimCoordinates: $4^{\circ} 6^{\prime} 34^{\prime \prime} \mathrm{S}, 41^{\circ} 43^{\prime} 39^{\prime \prime W}$; samplingProtocol: Pennsylvania light trap; verbatimEventDate: 19.iv.12; individualCount: 1; sex: male; lifeStage: adult; recordedBy: Takiya, D.M.; identifiedBy: Allan Paulo Moreira dos Santos; institutionCode: DZRJ; basisOfRecord: PreservedSpecimen

c. country: Brazil; stateProvince: Piauí; municipality: Piracuruca; locality: Parque Nacional de Sete Cidades, Riacho da Piedade; maximumElevationInMeters: 169; verbatimCoordinates: $4^{\circ} 6^{\prime} 34$ "S, $41^{\circ} 43^{\prime} 39$ "W; samplingProtocol: Pennsylvania light trap; verbatimEventDate: 19.iv.12; individualCount: 1; sex: female; lifeStage: adult; recordedBy: Takiya, D.M.; identifiedBy: Allan Paulo Moreira dos Santos; institutionCode: DZRJ; basisOfRecord: PreservedSpecimen

d. country: Brazil; stateProvince: Piauí; municipality: Piracuruca; locality: Parque Nacional de Sete Cidades, Riacho da Piedade; maximumElevationInMeters: 169; verbatimCoordinates: $4^{\circ} 6^{\prime} 34$ "S, $41^{\circ} 43^{\prime} 39 " \mathrm{~W}$; samplingProtocol: Malaise intercept trap; 
verbatimEventDate: 21.iv.12; individualCount: 4; sex: male; lifeStage: adult; recordedBy: Rafael, J.A. | Limeira-de-Oliveira, F. | Takiya, D.M. | et al.; identifiedBy: Allan Paulo Moreira dos Santos; institutionCode: DZRJ; basisOfRecord: PreservedSpecimen

\section{Cernotina sp. 2*}

\section{Materials}

a. country: Brazil; stateProvince: Piauí; municipality: Piracuruca; locality: Parque Nacional de Sete Cidades, Riacho da Piedade; maximumElevationInMeters: 169; verbatimCoordinates: $4^{\circ} 6^{\prime} 34$ "S, $41^{\circ} 43^{\prime} 39 " \mathrm{~W}$; samplingProtocol: Malaise intercept trap; verbatimEventDate: 18.iv.12; individualCount: 1; sex: male; lifeStage: adult; recordedBy: Rafael, J.A. | Limeira-de-Oliveira, F. | Takiya, D.M. | et al.; identifiedBy: Allan Paulo Moreira dos Santos; institutionCode: DZRJ; basisOfRecord: PreservedSpecimen

b. country: Brazil; stateProvince: Piauí; municipality: Piracuruca; locality: Parque Nacional de Sete Cidades, Riacho da Piedade; maximumElevationInMeters: 169; verbatimCoordinates: $4^{\circ} 6^{\prime} 34$ "S, $41^{\circ} 43^{\prime} 39$ "W; samplingProtocol: Malaise intercept trap; verbatimEventDate: 21.iv.12; individualCount: 2; sex: male; lifeStage: adult; recordedBy: Rafael, J.A. | Limeira-de-Oliveira, F. | Takiya, D.M. | et al.; identifiedBy: Allan Paulo Moreira dos Santos; institutionCode: DZRJ; basisOfRecord: PreservedSpecimen

c. country: Brazil; stateProvince: Piauí; municipality: Piracuruca; locality: Parque Nacional de Sete Cidades, Riacho da Piedade; maximumElevationInMeters: 169; verbatimCoordinates: $4^{\circ} 6^{\prime} 34$ "S, 41 ${ }^{\circ} 43^{\prime} 39$ "W; samplingProtocol: Malaise intercept trap; verbatimEventDate: 21.iv.12; individualCount: 2; sex: female; lifeStage: adult; recordedBy: Rafael, J.A. | Limeira-de-Oliveira, F. | Takiya, D.M. | et al.; identifiedBy: Allan Paulo Moreira dos Santos; institutionCode: DZRJ; basisOfRecord: PreservedSpecimen

d. country: Brazil; stateProvince: Piauí; municipality: Piracuruca; locality: Parque Nacional de Sete Cidades, Riacho da Piedade; maximumElevationInMeters: 169; verbatimCoordinates: $4^{\circ} 6^{\prime} 34$ "S, $41^{\circ} 43^{\prime} 39 " \mathrm{~W}$; samplingProtocol: Malaise intercept trap; verbatimEventDate: 21.iv.12; individualCount: 4; sex: male; lifeStage: adult; recordedBy: Rafael, J.A. | Limeira-de-Oliveira, F. | Takiya, D.M. | et al.; identifiedBy: Allan Paulo Moreira dos Santos; institutionCode: DZRJ; basisOfRecord: PreservedSpecimen

e. country: Brazil; stateProvince: Piauí; municipality: Piracuruca; locality: Parque Nacional de Sete Cidades, Riacho da Piedade; maximumElevationInMeters: 169; verbatimCoordinates: $4^{\circ} 6^{\prime} 34^{\prime \prime S}, 41^{\circ} 43^{\prime} 39 " \mathrm{~W}$; samplingProtocol: Malaise intercept trap; verbatimEventDate: 21.iv.12; individualCount: 3; sex: female; lifeStage: adult; recordedBy: Rafael, J.A. | Limeira-de-Oliveira, F. | Takiya, D.M. | et al.; identifiedBy: Allan Paulo Moreira dos Santos; institutionCode: DZRJ; basisOfRecord: PreservedSpecimen

f. country: Brazil; stateProvince: Piauí; municipality: Piracuruca; locality: Parque Nacional de Sete Cidades, Riacho da Piedade; maximumElevationInMeters: 169; verbatimCoordinates: $4^{\circ} 6^{\prime} 34^{\prime \prime S}, 41^{\circ} 43^{\prime} 39 " \mathrm{~W}$; samplingProtocol: Malaise intercept trap; verbatimEventDate: 21.iv.12; individualCount: 67; sex: male; lifeStage: adult; recordedBy: Rafael, J.A. | Limeira-de-Oliveira, F. | Takiya, D.M. | et al.; identifiedBy: Allan Paulo Moreira dos Santos; institutionCode: DZRJ; basisOfRecord: PreservedSpecimen 


\section{Genus Cyrnellus Banks, 1913}

Notes: New genus record for PI.

\section{Cyrnellus fraternus (Banks, 1915)}

\section{Materials}

a. country: Brazil; stateProvince: Piauí; municipality: Piracuruca; locality: Parque Nacional de Sete Cidades, Cachoeira do Riachão; maximumElevationInMeters: 171; verbatimCoordinates: $4^{\circ} 6^{\prime} 28^{\prime \prime S}, 41^{\circ} 40^{\prime} 13 " \mathrm{~W}$; samplingProtocol: Pennsylvania light trap; verbatimEventDate: 10.ii.13; individualCount: 1; sex: male; lifeStage: adult; recordedBy: Santos, A.P.M. | Takiya, D.M.; identifiedBy: Allan Paulo Moreira dos Santos; institutionCode: DZRJ; basisOfRecord: PreservedSpecimen

b. country: Brazil; stateProvince: Piauí; municipality: Piracuruca; locality: Parque Nacional de Sete Cidades, Cachoeira do Riachão; maximumElevationInMeters: 171; verbatimCoordinates: $4^{\circ} 6^{\prime} 28$ "S, $41^{\circ} 40^{\prime} 13^{\prime \prime W}$; samplingProtocol: Pennsylvania light trap; verbatimEventDate: 10.ii.13; individualCount: 5; sex: female; lifeStage: adult; recordedBy: Santos, A.P.M. | Takiya, D.M.; identifiedBy: Allan Paulo Moreira dos Santos; institutionCode: DZRJ; basisOfRecord: PreservedSpecimen

c. country: Brazil; stateProvince: Piauí; municipality: Piracuruca; locality: Parque Nacional de Sete Cidades, Poço do Bananeira; maximumElevationInMeters: 158;

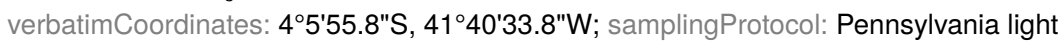
trap; verbatimEventDate: 10.ii.13; individualCount: 8; sex: male; lifeStage: adult; recordedBy: Santos, A.P.M. | Takiya, D.M.; identifiedBy: Allan Paulo Moreira dos Santos; institutionCode: DZRJ; basisOfRecord: PreservedSpecimen

d. country: Brazil; stateProvince: Piauí; municipality: Piracuruca; locality: Parque Nacional de Sete Cidades, Poço do Bananeira; maximumElevationInMeters: 158; verbatimCoordinates: $4^{\circ} 5^{\prime} 55.8^{\prime \prime S}, 41^{\circ} 40^{\prime} 33.8 \mathrm{~W}$; samplingProtocol: Pennsylvania light trap; verbatimEventDate: 10.ii.13; individualCount: 2; sex: female; lifeStage: adult; recordedBy: Santos, A.P.M. | Takiya, D.M.; identifiedBy: Allan Paulo Moreira dos Santos; institutionCode: DZRJ; basisOfRecord: PreservedSpecimen

e. country: Brazil; stateProvince: Piauí; municipality: Piracuruca; locality: Parque Nacional de Sete Cidades, Riacho da Bananeira; maximumElevationInMeters: 189;

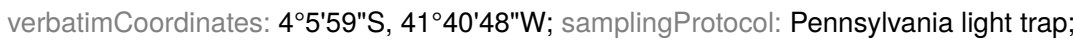
verbatimEventDate: 18.iv.12; individualCount: 2; sex: male; lifeStage: adult; recordedBy: Takiya, D.M.; identifiedBy: Allan Paulo Moreira dos Santos; institutionCode: DZRJ; basisOfRecord: PreservedSpecimen

f. country: Brazil; stateProvince: Piauí; municipality: Piracuruca; locality: Parque Nacional de Sete Cidades, Riacho da Bananeira; maximumElevationInMeters: 189;

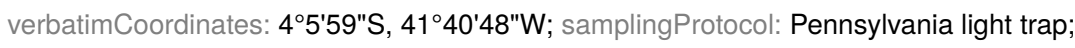
verbatimEventDate: 18.iv.12; individualCount: 2; sex: female; lifeStage: adult; recordedBy: Takiya, D.M.; identifiedBy: Allan Paulo Moreira dos Santos; institutionCode: DZRJ; basisOfRecord: PreservedSpecimen

g. country: Brazil; stateProvince: Piauí; municipality: Piracuruca; locality: Parque Nacional de Sete Cidades, Riacho da Bananeira; maximumElevationInMeters: 189; verbatimCoordinates: $4^{\circ} 5^{\prime} 59^{\prime \prime S}, 41^{\circ} 40^{\prime} 48^{\prime \prime} \mathrm{W}$; samplingProtocol: Malaise intercept trap; verbatimEventDate: 19.iv.12; individualCount: 5; sex: male; lifeStage: adult; recordedBy: Rafael, J.A. | Limeira-de-Oliveira, F. | Takiya, D.M. | et al.; identifiedBy: Allan Paulo Moreira dos Santos; institutionCode: DZRJ; basisOfRecord: PreservedSpecimen 
h. country: Brazil; stateProvince: Piauí; municipality: Piracuruca; locality: Parque Nacional de Sete Cidades, Riacho da Bananeira; maximumElevationInMeters: 189; verbatimCoordinates: $4^{\circ} 5^{\prime} 59$ "S, $41^{\circ} 40^{\prime} 48$ "W; samplingProtocol: Malaise intercept trap; verbatimEventDate: 19.iv.12; individualCount: 1; sex: female; lifeStage: adult; recordedBy: Rafael, J.A. | Limeira-de-Oliveira, F. | Takiya, D.M. | et al.; identifiedBy: Allan Paulo Moreira dos Santos; institutionCode: DZRJ; basisOfRecord: PreservedSpecimen

i. country: Brazil; stateProvince: Piauí; municipality: Piracuruca; locality: Parque Nacional de Sete Cidades, Cachoeira do Riachão; maximumElevationInMeters: 171; verbatimCoordinates: $4^{\circ} 6^{\prime} 28^{\prime \prime S}, 41^{\circ} 40^{\prime} 13 " \mathrm{~W}$; samplingProtocol: Pennsylvania light trap; verbatimEventDate: 8.ii.13; individualCount: 1; sex: male; lifeStage: adult; recordedBy: Santos, A.P.M. | Takiya, D.M.; identifiedBy: Allan Paulo Moreira dos Santos; institutionCode: DZRJ; basisOfRecord: PreservedSpecimen

j. country: Brazil; stateProvince: Piauí; municipality: Piracuruca; locality: Parque Nacional de Sete Cidades, Poço do Bananeira; maximumElevationInMeters: 158; verbatimCoordinates: $4^{\circ} 5^{\prime} 55.8^{\prime \prime S}, 41^{\circ} 40^{\prime} 33.8^{\prime \prime} \mathrm{W}$; samplingProtocol: Pennsylvania light trap; verbatimEventDate: 9.ii.13; individualCount: 3; sex: male; lifeStage: adult; recordedBy: Santos, A.P.M. | Takiya, D.M.; identifiedBy: Allan Paulo Moreira dos Santos; institutionCode: DZRJ; basisOfRecord: PreservedSpecimen

k. country: Brazil; stateProvince: Piauí; municipality: Piracuruca; locality: Parque Nacional de Sete Cidades, Poço do Bananeira; maximumElevationInMeters: 158; verbatimCoordinates: $4^{\circ} 5^{\prime} 55.8^{\prime \prime S}, 41^{\circ} 40^{\prime} 33.8^{\prime \prime} \mathrm{W}$; sampling Protocol: Pennsylvania light trap; verbatimEventDate: 9.ii.13; individualCount: 1; sex: female; lifeStage: adult; recordedBy: Santos, A.P.M. | Takiya, D.M.; identifiedBy: Allan Paulo Moreira dos Santos; institutionCode: DZRJ; basisOfRecord: PreservedSpecimen

Distribution: USA. Mexico. El Salvador. Nicaragua. Costa Rica. Panama. Venezuela. Suriname. Brazil: PA, AM, PI!, BA, MG, MS, ES, RJ, PR, SC. Ecuador. Paraguay. Argentina. Uruguay.

Notes: New species record for PI.

\section{Genus Polyplectropus Ulmer, 1905}

Notes: New genus record for PI.

\section{Polyplectropus rondoniensis Chamorro \& Holzenthal, 2010}

\section{Material}

a. country: Brazil; stateProvince: Piauí; municipality: Piracuruca; locality: Parque Nacional de Sete Cidades, Riacho da Piedade; maximumElevationInMeters: 169; verbatimCoordinates: $4^{\circ} 6^{\prime} 34$ "S, $41^{\circ} 43^{\prime} 39$ "W; samplingProtocol: Malaise intercept trap; verbatimEventDate: 21.iv.12; individualCount: 1; sex: male; lifeStage: adult; recordedBy: Rafael, J.A. | Limeira-de-Oliveira, F. | Takiya, D.M. | et al.; identifiedBy: Allan Paulo Moreira dos Santos; institutionCode: DZRJ; basisOfRecord: PreservedSpecimen

Distribution: Brazil: PI!, RO.

Notes: New species record for Northeastern Brazil. 


\section{Family Xiphocentronidae}

Notes: New family record for PI.

\section{Genus Xiphocentron Brauer, 1870}

Notes: New genus record for PI.

\section{Xiphocentron sp. 2}

\section{Materials}

a. country: Brazil; stateProvince: Piauí; municipality: Piracuruca; locality: Parque Nacional de Sete Cidades, Cachoeira do Riachão; maximumElevationInMeters: 171; verbatimCoordinates: $4^{\circ} 6^{\prime} 28^{\prime \prime} \mathrm{S}, 41^{\circ} 40^{\prime} 13^{\prime \prime} \mathrm{W}$; samplingProtocol: Manual; verbatimEventDate: 11.ii.13; individualCount: 9; lifeStage: immature; recordedBy: Santos, A.P.M. | Takiya, D.M.; identifiedBy: Allan Paulo Moreira dos Santos; institutionCode: DZRJ; basisOfRecord: PreservedSpecimen

b. $\quad$ country: Brazil; stateProvince: Piauí; municipality: Piracuruca; locality: Parque Nacional de Sete Cidades, Cachoeira do Riachão; maximumElevationInMeters: 171; verbatimCoordinates: $4^{\circ} 6^{\prime} 28^{\prime \prime} \mathrm{S}, 41^{\circ} 40^{\prime} 13^{\prime \prime} \mathrm{W}$; samplingProtocol: Manual; verbatimEventDate: 9.ii.13; individualCount: 23; lifeStage: immature; recordedBy: Santos, A.P.M. | Takiya, D.M.; identifiedBy: Allan Paulo Moreira dos Santos; institutionCode: DZRJ; basisOfRecord: PreservedSpecimen

\section{Discussion}

\section{Species shared with other phytogeographical domains}

Although the Caatinga shares with Cerrado similar landscapes, in part due its savannahlike formation and their geographical proximity, under a spatial evolution standpoint historically the biota from Northeastern Brazil forested areas are largely linked to Amazonia and Atlantic Forest (see Santos et al. 2007) and even appears disjunct from other savannah-like South American formations such as the Bolivian Chaco (Werneck et al. 2012). Considering the better known Odonata, the composition of the dragonfly assemblage is remarkable by virtually lacking typical Cerrado species and mixing elements from forested biomes of Amazonia and Atlantic Forest domains. Sampling from the two National Parks comprises largely common species occurring in lentic environments of almost all American countries (e.g., Ischnura capreolus at PNSC and Erythrodiplax fusca at PNU), while a smaller number of species were only previously known from Amazonia (e.g., Castoraeschna corbeti at PNU; Carvalho et al. 2009) or from Atlantic Forest (e.g., Neocordulia setifera at PNU; Pinto and Lamas 2010). Some of them are largely common and characteristic of these domains, such as $P$. complicatus in the Atlantic Forest (see distribution data in De Almeida et al. 2013).

Past connections between Amazonia and Atlantic Forest biotas are open to debate, hypotheses diverging if they occurred during the Miocene through South America's dry 
diagonal vegetation, including currently the Cerrado and Chaco formations, or during the Plio-Pleistocene through forested areas in the Caatinga Domain (see references in Batalha-Filho et al. 2013). Furthermore, wet period intervals every $20,000-200,000$ years during at least 2 million years created intermittent corridors of between forested areas in Brazil (Wang et al. 2004) and can explain the mixing of fauna among Amazonia-CaatingaAtlantic Forest. At least the PNU can be considered an island of humid forest surrounded by savannah-like formation and studies focusing the aquatic insect assemblages can strongly help understand the spatial evolution of these forested patches in the Northeastern Brazil and past connections between the two largest tropical forest domains in South America.

\section{New distributional records and notes on species records}

Based on the collected material during this project, one caddisfly species was firstly recorded from Brazil, Phylloicus pirapo (Calamoceratidae) previously known from Argentina and Paraguay. Eighteen species collected at PNU and 21 at PNSC (5 species shared between parks) represent their first record for Northeastern Brazil.

Several new state records are made based on this material, especially for Piauí State. Thirty and 56 species are firstly recorded from Ceará and Piauí states, respectively. These exclude records previously published, but based on material collected during this project, which add other 10 new species records for these states (see Table 2). Herein, it is also recorded 25 and 47 new genera and 9 and 14 aquatic insect families from Ceará and Piauí states, respectively.

Some of these new records are for widespread species in South America, such as for many dragonflies (see below), however, others significantly expand species ranges in Brazil. For example, the caddisflies Helicopsyche monda (Helicopsychidae) and Chimarra calori (Philopotamidae) previously known from Southeastern and/or Southern Brazil have their ranges expanded for over $1,000 \mathrm{~km}$.

The knowledge of stoneflies from Northeastern Brazil is still very scarce, when compared to the other aquatic insects studied. In the Neotropical Plecoptera catalogue, only two species, Anacroneuria lacunosa (Navás, 1926) and A. parilobata Klapálek, 1922, from this Brazilian Region was recorded (Froehlich 2010), both from Bahia State. In the last ten years, following an increase of local taxonomists and inventories, species number boosted from two to 27 stoneflies (Lecci and Froehlich 2011, Righi-Cavallaro et al. 2013, Duarte et al. 2014, Duarte and Lecci 2014, Lecci et al. 2014, Duarte and Lecci 2016). However, most sampling localities are still concentrated in some regions of Bahia State. Nevertheless, stonefly diversity still seems higher in the Atlantic forest, than in the Caatinga. A single Anacroneuria species has been recently described fom PNU (Duarte and Lecci 2016) and was recorded herein from both National parks, thus representing the first record of Plecoptera from Piauí State.

Except by few Amazonian and Atlantic Forest representatives, most dragonflies correspond to common species and are now firstly recorded mainly due to undersampling. Thus, field 
surveys must be one of the priority efforts for assessment of the diverse dragonfly fauna of tropical South America (e.g., Pinto and Lamas 2010). Amongst the new records, the known distribution of Acanthagrion jessei (Coenagrionidae) was extended considerably from previous localities at Brazilian Amazonia and Pantanal. Another unexpected occurrence is of the South American emerald Neocordulia setifera (Anisoptera, incertae sedis), a species apparently confined to ombrophilous forested areas in the Atlantic Forest Domain with several misidentified specimens cited in the literature, including records from Brazil (MT, GO, MG), and Ecuador (APP, in prep.). Other specimens need further studies, such as, a possible new species of the Oxyagrion basale-group (Coenagrionidae) from PNSC; a single female with mesostigmal plate and mesepisternal fossae similar to that of the Central American Acanthagrion quadratum (not a well preserved specimen) from PNU; and specimens of Argia, a genus of difficult species identification, which are broken or smashed, thus were determined doubtfully ("cf."). Specimens from PNU of the Amazonian Castoraeschna corbeti (Aeshnidae) and Hetaerina indeprensa (Calopterygidae), species previously only known from their type localities in Pará State (Brazil) are slightly distinct from typical specimens and need further studies to investigate if they only represent geographical variation.

Several published records of dragonfly species from the states of CE and PI are old, based on misidentifications or wrong localities, or for names currently under a different taxonomic status, and as a result, some of them should be ignored or are pending confirmation. Such cases are discussed below.

The Amazonian damselflies Mnesarete cupraea (Selys, 1853) and Metaleptobasis bicornis (Selys, 1877) were recorded from Ceará State (Garrison 2006: 21, von Ellenrieder 2013: 20; respectively) based on a misinterpretation of the locality of "Canindé" on the Rio Gurupi Basin at the border of Pará and Maranhão states (see Pinto and Lamas 2011). The occurrence of $M$. cupraea in Ceará was recently proven to be true for Serra da Ibiapaba ( Table 2).

Belle 1983: 170-171), in his revision of Zonophora, provided a map where Zonophora calippus calippus Selys, 1869 and Zonophora batesi Selys, 1869 are apparently recorded from Piauí State, however it is clear that their location was based on the material from Maranhão cited by Belle 1972: 236-236). This mistake was reproduced by Garrison et al. 2006: 131) in their thumbnail map. Additionally, several other thumbnail maps for genera (Garrison et al. 2006) show ranges of distribution in both Ceará and Piauí States (e.g., Phyllogomphoides, Aeschnosoma, and Ypiranthemis), however, we have not located references for these records and they should be considered potential distributions rather than actual occurrences. Even considering that species of some of these genera are common and widespread in South America, they are pending confirmation based on voucher specimens.

In the first published list of dragonflies from Ceará State, Navás (1924) cited currently considered dubious or questionable names due to changes in species concepts and very likely also based on misidentifications. These species were not included in Table 2. Navás (1924) cited Erythrodiplax connata (Burmeister, 1839), which likely refers to E. fusca since 
the former is restricted to Chile (Paulson 2003). Erythrodiplax nigricans was mentioned dubiously in the generic revision by Borror (1942), and based on its distribution in southern South America with the northernmost record from Rio de Janeiro State (Anjos-Santos and Costa 2006), it seems unlikely that it occurs in Northeastern Brazil. Three other species recorded by Navás (1924), Micrathyria eximia Kirby, 1897, Orthemis ferruginea (Fabricius, 1775), and Perithemis domitia (Drury, 1773), are very likely misidentifications. While reviewing $M$. eximia, Westfall (1992) observed that almost all specimens identified as this species were misidentified, including most Brazilian ones, as they indeed correspond to four species. Orthemis ferruginea is a largely North and Central American species with southernmost records to Costa Rica (Donnelly 1995), and its occurrence in South America is very unlikely. Material in which this record was based represent at least five species of the O. ferruginea group (see Pinto 2010). Finally, P. domitia is a Caribbean and Central America species with southernmost records in Venezuela and Colombia. Costa et al. (2006) considered all records of the latter species from Brazil as records of $P$. mooma and we agree with these authors.

\section{Increase of the known aquatic insect diversity of Ceará and Piauí states}

Besides the high number of taxa recorded for the first time from Ceará and Piauí, effectively increasing the knowledge of aquatic insect diversity for these states, several undescribed species were detected. Twenty-six and 20 undescribed species from PNU and PNSC, respectively, were detected based on the material collected during this project, of those 12 from PNU (Câmara et al. 2015, Henriques-Oliveira and Santos 2014, Souza et al. 2014a, Souza et al. 2014b, Santos et al. 2016a) and four from PNSC (Cordeiro et al. 2014, Souza et al. 2014b, Souza et al. 2016a, Souza et al. 2016b) have already been described.

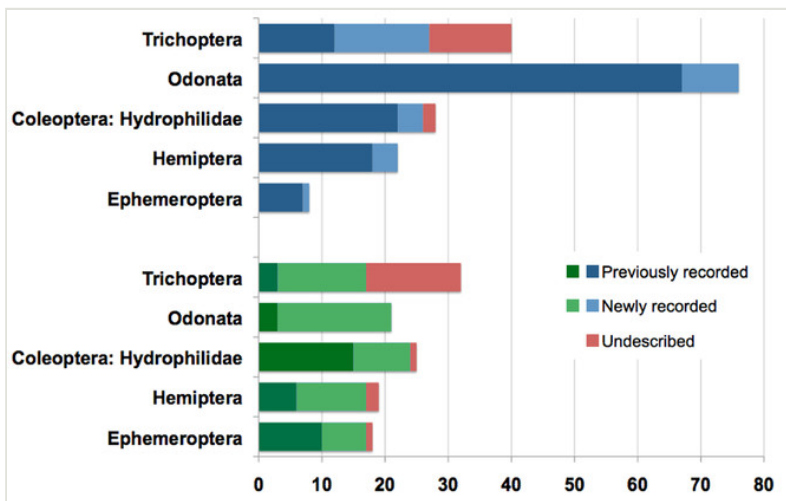

Figure 29.

Number of previously recorded species of Trichoptera, Odonata, Hemiptera, Ephemeroptera, and Hydrophilidae (Coleoptera) for Ceará (blue) and Piauí (green). Dark blue or green represent the number of previously recorded species based on the literature. Light blue and green represent the number of species recorded for the first time in its respective state based on material collected at Ubajara (CE) and Sete Cidades (PI) National Parks. Number of undescribed species collected in these parks are given in red. 
Many more aquatic insect species (except of Ephemeroptera) have been recorded from Ceará than Piauí (see Table 2). Our results represent an increase of approximately $16 \%$ in the number of known species for Ceará State, except for Trichoptera (Fig. 29). Caddisflies (the most diverse of groups sampled) seem to be understudied in both Brazilian states, with our sampling including a high proportion of species still to be described and results representing an increase of approximately $70 \%$ in the number of species known for Ceará and of $91 \%$ for Piauí. This increase for Piauí State is especially evident in other groups, such as, for Coleoptera, Ephemeroptera, and Hemiptera, which increased in approximately $51 \%$ of known species. This increase is even higher (86\%) for dragonflies (Odonata) in Piauí, which is surprising given that they are possibly the most well-studied of focal groups.

\section{Species richness comparison between Parque Nacional de Ubajara (PNU) and Parque Nacional de Sete Cidades (PNSC)}

There are no published lists of insect species for neither Parque Nacional de Ubajara (PNU) nor Parque Nacional de Sete Cidades (PNSC). In PNU's management plan it is cited the occurrence of five orders and 14 insect families, with 6 genera and 9 species identified. However, these names were not made available. In another study, Silva and Ferreira (2009) list 26 families of 9 insect orders, including the identification solely of the genera Conicera (Phoridae), Lutzomyia (Psychodidae), and Endecous (Phalangopsidae), as part of a cave invertebrate inventory of PNU. The only insect faunal study published involving the two parks is a list of Lutzomyia (Diptera: Psychodidae) species by Martins et al. (1989), with five species occurring at PNU and seven species ate PNSC, while only Lutzomyia (L.) longipalpis (Lutz \& Neiva, 1912) occurred in both parks. Thus, this preliminary list of aquatic insect taxa already represents a good sampling of insect diversity for these parks and a baseline for further monitoring and conservation strategies of freshwater macroinvertebrate fauna.

Considering all focal taxa, only $11 \%$ of species were found in both National Parks (Fig. 18), their species composition displaying very high complementarity (89\% species). Although both parks are relatively close to eachother and within the Caatinga Domain, they drastically differ in phytophysiography. Specifically in the case of aquatic insects, the low complementary is more probably explained by the structural differences of water bodies sampled available in the different parks. At PNSC most part of aquatic insects (67 species) were collected at Cachoeira do Riachão (Fig. 3), a (supposedly) permanent stream with broad humid gallery forest and high diversity of microhabitats (sand, litter, and rocks), and in some temporary streams in open savannah vegetation (Fig. 4), which were partially dry during the second expedition and with lentic characteristics. The majority of water bodies sampled at PNU were very homogeneous, bedrock rapid streams and low diversity of microhabitats.

Although for some groups, e.g. Trichoptera, the number of species collected in each National Park was similar, rarefaction curves based on individuals sampled (Fig. 30) surprisingly suggest much higher expected richness of aquatic insect species at PNSC than at PNU, thus much more sampling is needed at PNSC to have a better estimate of 
total species richness. This is certainly the result of a higher range of aquatic microhabitats sampled at PNSC, as discussed above. However, it is still surprising that such a high diversity was found at PNSC in this study, given that the second expedition to these parks was conducted at the start of the rainy season, when some bodies of water sampled in the first expedition to PNSC (e.g., stream below Cachoeira do Riachão) were completely dry and resulted in the collection of a low number of individuals. Insect abundances in general seem to conspicuously peak during the rainy season and follow a very strong seasonality in Caatinga areas (Vasconcellos et al. 2010), which probably affected our effective sampling of a much higher richness from PNSC.
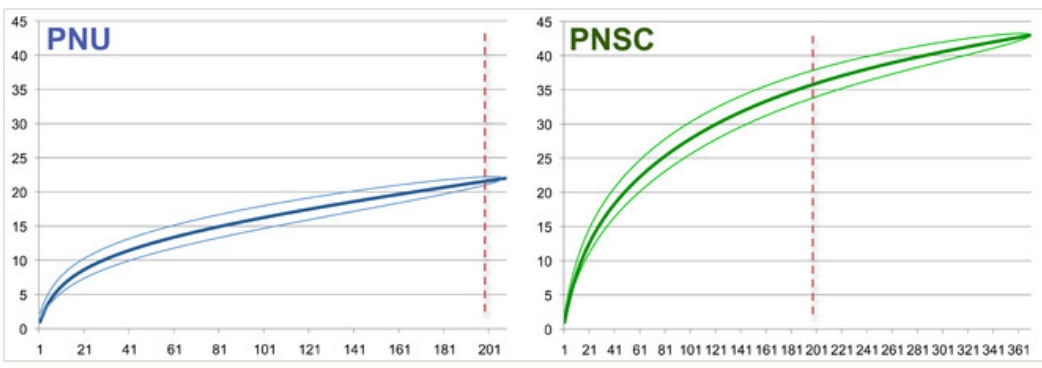

Figure 30.

Rarefaction curves (thick lines) of aquatic insect richness from Ubajara (PNU, blue) and Sete Cidades (PNSC, green) National Parks with 95\% confidence limits (fine lines). Dashed red line indicates the sample point of 200 individuals.

\section{Acknowledgements}

Specimens were collected as part of a project coordinated by JAR and financed by Conselho Nacional de Desenvolvimento Científico e Tecnológico (CNPq, proc. 551.991/2011-9) and Instituto Chico Mendes de Conservação da Biodiversidade (ICMBio) through the program "Pesquisa em Unidades de Conservação do Bioma Caatinga". Local logistic support for collecting trips was given by F. Limeira-de-Oliveira (Universidade Estadual do Maranhão) and his students. Rodney Cavichioli (Universidade Federal do Paraná) and Alexandre Somavilla (INPA) assisted placing Pennsylvania traps in the field. Collecting permits in both National Parks were granted by ICMBio (SISBIO \#32560). DMT and JAR are research productivity fellows from CNPq (procs. 306.897/2014-8 and 300.305/2007-9) and DMT is a Jovem Cientista do Nosso Estado fellow (Fundação Carlos Chagas Filho de Amparo à Pesquisa do Estado do Rio de Janeiro, proc. E-26/202.786/2015). APP is a post-doctoral fellow from CNPq (PDJ procs. 151.122/2013-0 and 157.592/2015-4). Gabriela Jardim (UFRJ) and Rosser W. Garrison (California Department of Food and Agriculture) have confirmed identifications of Leptonema and Argia specimens. A previous version of this manuscript benefited greatly from revisions of Rafael Boldrini (Universidade Federal de Roraima) e Danielle Anjos-Santos (Centro de Investigación Esquel de Montaña y Estepa Patagónica). 


\section{Author contributions}

APMS, ALHO, ALC, APP, BHLS, BC, FFFM, FAC, ICG, IRSC, JTC, JFB, WRMS identified and quantified material and provided distributional data for species cited. DMT, APMS, JAR, conceived project and collected material. DMT, APMS, APP wrote paper.

\section{References}

- Anjos-Santos D, Costa J (2006) A revised checklist of Odonata (Insecta) from Marambaia, Rio de Janeiro, Brazil with eight new records. Zootaxa 1300: 37-50.

- Assis CV, Costa JM (1994) Seis novas larvas do gênero Micrathyria Kirby e notas sobre a distribuição no Brasil (Odonata, Libellulidae). Revista Brasileira de Zoologia 11 (2): 195-209. DOI: $10.1590 / \mathrm{s} 0101-81751994000200003$

- $\quad$ Barbosa JF, Nessimian JL (2013) New species and new records of Notonecta (Hemiptera: Heteroptera: Notonectidae) from Brazil. Zoologia (Curitiba) 30 (6): 692-696. DOI: $10.1590 /$ s1984-46702013005000011

- Barbosa JF, Rodrigues HDD (2013) A new species of Martarega White, 1879, with new distributional records of Notonectidae (Hemiptera: Heteroptera: Nepomorpha) from Brazil. Zootaxa 3682 (4): 534. DOI: 10.11646/zootaxa.3682.4.4

- $\quad$ Batalha-Filho H, Fjeldså J, Fabre P, Miyaki CY (2013) Connections between the Atlantic and the Amazonian forest avifaunas represent distinct historical events. Journal of Ornithology 154 (1): 41-50. DOI: 10.1007/s10336-012-0866-7

- Belle J (1972) Further studies on South American Gomphidae (Odonata). Tijdschrift voor Entomologie 115 (5): 217-240.

- Belle J (1983) A review of the genus Zonophora Selys (Odonata, Gomphidae). Tijdschrift Voor Entomologie 126: 145-173.

- Boeger WA, Zaher H, Rafael JA, Valim MP (2016) Catálogo Taxonômico da Fauna do Brasil. http://fauna.jbri.gov.br/fauna/listaBrasil/ConsultaPublicaUC. Accession date: 2016125.

- $\quad$ Boldrini R, Cruz PV, Salles FF, Belmont EL, Hamada N (2012) Baetidae (Insecta: Ephemeroptera) from northeastern Brazil. Checklist 8 (1): 88-94. URL: http:// www.checklist.org.br/getpdf?SL105-11

- $\quad$ Borror DJ (1942) A revision of Libellulinae genus Erythrodiplax (Odonata). Contributions in Zoology and Entomology 4: 1-286.

- Câmara JT, Takiya DM, Plant AR, Rafael JA (2015) Neotropical Hemerodromia Meigen (Diptera: Empididae), a world of discovery II: New species from Atlantic forest, Brazil. Zootaxa 4028 (2): 197-214. DOI: 10.11646/zootaxa.4028.2.2

- $\quad$ Carvalho A, Pinto Â, Ferreira-Jr N (2009) Castoraeschna corbeti sp. nov. from Floresta Nacional de Carajás, Pará state, Brazil (Odonata: Aeshnidae). International Journal of Odonatology 12 (2): 337-346. DOI: $10.1080 / 13887890.2009 .9748350$

- $\quad$ Carvalho J, Bravo F (2014) Odonata do semiárido. p. 83-89. In: Bravo F, Calor A (Orgs). Artrópodes do Semiárido: biodiversidade e conservação. Printmídia, Feira de Santana, $289 \mathrm{pp}$.

- $\quad$ Castelletti CHM, Santos AMM, Tabarelli M, da Silva J (2003) Quanto ainda resta da Caatinga? Uma estimativa preliminar. In: Leal IR, Tabarelli M, da Silva JMC (Eds) 
Ecologia e Conservação da Caatinga. Universidade Federal de Pernambuco, Recife, $822 \mathrm{pp}$.

- Chapman RF (1998) The Insects. Structure and Function. Cambridge University Press, Cambridge, $770 \mathrm{pp}$.

- $\quad$ Cheng L (1976) Marine insects. North-Holland Publishing Company, Amsterdam, 581 pp.

- Colwell R, Coddington J (1994) Estimating terrestrial biodiversity through extrapolation. Philosophical Transactions of the Royal Society (Series B) 345: 101-118. DOI: 10.1098/ rstb.1994.0091

- $\quad$ Cordeiro IRS, Moreira FFF (2015) New distributional data on aquatic and semiaquatic bugs (Hemiptera: Heteroptera: Gerromorpha \& Nepomorpha) from South America. Biodiversity Data Journal 3: 4913-4913. DOI: 10.3897/BDJ.3.e4913

- $\quad$ Cordeiro IRS, Moreira FFF, Silva FAC (2014) A new Ochterus (Hemiptera: Heteroptera: Ochteridae) from northeastern Brazil, with a key to the species recorded from the country. Zootaxa 3860 (5): 493-497. DOI: 10.11646/zootaxa.3860.5.8

- Costa AM, Quinteiro FB, Calor AR (2014) Trichoptera do Semiárido I: Annulipalpia. p. 225-228. In: Bravo F, Calor A (Orgs). Artrópodes do Semiárido: biodiversidade e conservação. Printmídia, Feira de Santana, 289 pp.

- $\quad$ Costa JM, Santos TC (1997) Intra and interspecific variation in the genus Uracis Rambur, 1842, with a key to the known species (Anisoptera: Libellulidae). Odonatologica 26 (1): 1-7.

- Costa JM, Souza LOI, Muzón J (2006) Descriptions of three new species of Odonata from Brazil. Zootaxa 134: 53-68.

- Coutinho LM (2006) O conceito de bioma. Acta Botanica Brasiliense 20 (1): 13-23. DOI: 10.1590/S0102-33062006000100002

- $\quad$ Cruz PV, Salles FF, Hamada N (2014) Callibaetis Eaton (Ephemeroptera: Baetidae) from Brazil. Journal of Natural History 48: 591-660. DOI: 10.1080/00222933.2013.7918 $\underline{83}$

- $\quad$ Cruz PV, Belmont EL, Boldrini R, Hamada N (2011) Leptohyphidae (Insecta: Ephemeroptera) from northeastern Brazil. Neotropical Entomology 40 (6): 682-688. DOI: $10.1590 / \mathrm{s} 1519-566 \times 2011000600008$

- De Almeida MVO, Pinto AP, Carvalho AL, Takiya DM (2013) When rare is just a matter of sampling: unexpected dominance of clubtail dragonflies (Odonata, Gomphidae) through different collecting methods at Parque Nacional da Serra do Cipó, Minas Gerais State, Brazil. Revista Brasileira de Entomologia 57 (4): 417-423. DOI: 10.1590/ s0085-56262013005000042

- $\quad$ Dias da Rocha F (1908) Insectos. Boletim do Museu Rocha 1 (1): 61-81.

- Dias da Rocha F (1936) Subsídios para o estudo da fauna cearense. III. Insecta. Nordeste Agrícola 1: 96-99.

- Dijkstra KB, Monaghan MT, Pauls SU (2014) Freshwater Biodiversity and Aquatic Insect Diversification. Annual Review of Entomology 59 (1): 143-163. DOI: 10.1146/annurevento-011613-161958

- Donnelly TW (1995) Orthemis ferruginea - An adventure in Caribbean biogeography. Argia 7 (4): 9-12.

- Duarte T, Lecci LS (2014) Stoneflies (Insecta: Plecoptera) from Serra Bonita, Bahia, Brazil: New species and updated records. Zootaxa 3779 (1): 81. DOI: 10.11646/ 
- $\quad$ Duarte T, Lecci LS (2016) New species and records of Anacroneuria (Plecoptera: Perlidae) from the northeastern semi-arid region of Brazil. Zootaxa 4079 (2): 291-300. DOI: $10.11646 /$ zootaxa.4079.2.10

- $\quad$ Duarte T, Bispo PC, Calor AR (2014) A new species of Tupiperla Froehlich, 1969 (Plecoptera: Gripopterygidae) from Serra da Jibóia, Bahia, Brazil. Zootaxa 3835 (1): 140. DOI: $10.11646 /$ zootaxa.3835.1.9

- Flint OSJ (1998) Studies of Neotropical Caddisflies, LIII: A taxonomic revision of the subgenus Curgia of the genus Chimarra (Trichoptera: Philopotamidae). Smithsonian Contributions to Zoology 594: 1-130. [In English]. DOI: 10.5479/si.00810282.594

- $\quad$ Franca D, Paprocki H, Calor AR (2013) The genus Macrostemum Kolenati 1859 (Trichoptera: Hydropsychidae) in the Neotropical Region: Description of two new species, taxonomic notes, distributional records and key to males. Zootaxa 3716 (3): 301-335. DOI: 10.11646/zootaxa.3716.3.1

- $\quad$ Froehlich CG (2010) Catalogue of Neotropical Plecoptera . Illiesia 6: 118-205.

- Frost SW (1957) The Pennsylvania insect light trap. Journal of Economic Entomology 50 (3): 287-292. DOI: 10.1093/jee/50.3.287

- Garrison RW (2006) A synopsis of the genera Mnesarete Cowley, Bryoplathanon gen. nov., and Ormenoplebia gen. nov. (Odonata: Calopterygidae). Contributions in Science 506: 1-84.

- $\quad$ Garrison RW, von Ellenrieder N, Louton JA (2006) Dragonfly genera of the New World: an illustrated and annotated key to the Anisoptera. The Johns Hopkins University Press, Baltimore, $368 \mathrm{pp}$.

- $\quad$ Gressitt JL, Gressitt MK (1962) An improved malaise trap. Pacific Insects 87: 87-90.

- $\quad$ Grimaldi D, Engel MS (2005) Evolution of the Insects. Cambridge University Press, New York, $755 \mathrm{pp}$.

- Hammer $\varnothing$, Harper DAT, Ryan PD (2001) PAST: Paleontological Statistics Software Package for Education and Data Analysis. Palaeontologia Electronica 4 (1): 9.

- Henriques-Oliveira AL, Santos APM (2014) Two new species of Atanatolica Mosely 1936 (Trichoptera: Leptoceridae) from Peru and Northeastern Brazil. Zootaxa 3869 (5): 537-547. DOI: 10.11646/zootaxa.3869.5.3

- Henriques-Oliveira AL, Dumas LL, Nessimian JL (2014) Three new species and new distributional records of Oecetis McLachlan 1877 (Trichoptera: Leptoceridae:

Leptocerinae) from Brazil. Zootaxa 3753 (3): 273-282. DOI: 10.11646/zootaxa.3753.3.6

- Hungerford HB (1948) The Corixidae of the Western Hemisphere (Hemiptera). University of Kansas Science Bulletin 32: 5-827.

- Hungerford HB (1954) The genus Rheumatobates Bergroth (Hemiptera-Gerridae). University of Kansas Science Bulletin 36: 529-588. DOI: 10.5962/bhl.part.24625

- Jäch MA, Balke M (2008) Global diversity of water beetles (Coleoptera) in freshwater. Developments in Hydrobiology 595: 419-442. DOI: 10.1007/978-1-4020-8259-7 43

- Keffer SL (1997) Systematics of the New World waterscorpion genus Curicta Stål (Heteroptera: Nepidae). Journal of the New York Entomological Society 104: 117-215.

- Kirby WF (1889) A revision of the subfamily Libellulinae, with descriptions of new genera and species. Transactions of the Zoological Society of London 12 (9): 249-348. DOI: $10.1111 / j .1096-3642.1889 . t b 00016 . x$

- Knisch A (1924) Neue neotropische Palpicornier (Col. Hydrophilidae. - Op. 16.). Wiener Entomologische Zeitung 41: 114-140. 
- Komarek A (2005) Taxonomic revision of Anacaena Thomson, 1859 II. Neotropical species (Coleoptera: Hydrophilidae). Koleopterologische Rundschau 75: 253-301.

- Lauck DR (1962) A monograph of the genus Belostoma (Hemiptera) Part I. Introduction to $B$. dentatum and subspinosum groups. Bulletin of the Chicago Academy of Sciences 11 (3): 34-81.

- Leal IR, Da Silva JMC, Tabarelli M, Lacher TE (2005) Changing the Course of Biodiversity Conservation in the Caatinga of Northeastern Brazil. Conservation Biology 19 (3): 701-706. DOI: 10.1111/i.1523-1739.2005.00703.X

- Lecci L, Froehlich CG (2016) Plecoptera in Catálogo Taxonômico da Fauna do Brasil. ht tp://fauna.jbri.gov.br/fauna/faunadobrasil/304. Accession date: 2016126.

- Lecci LS, Froehlich CG (2011) Taxonomic revision of Gripopteryx (Pictet, 1841) (Plecoptera: Gripopterygidae). Zootaxa 2792: 1-21.

- $\quad$ Lecci LS, Simões TVD, Calor AR (2014) Plecoptera do Semiárido: conhecimento atual e desafios. p. 91-98. In: Bravo F, Calor A (Orgs). Artrópodes do Semiárido: biodiversidade e conservação. Printmídia, Feira de Santana, 289 pp.

- Machado AM (2015) Perilestes eustaquioi sp. nov. and new distributional records of Perilestidae (Odonata) in Brazil. Zoologia 32 (5): 428-430. DOI: 10.1590/ s1984-46702015000500012

- Martins AV, Dias ES, Falcão AL, da Silva JE (1989) Notas sobre os flebótomos dos estados do Ceará e Piauí, com a descrição da fêmea de Lutzomyia samueli (Deane, 1955) (Diptera, Psychodidae, Phlebotominae). Memórias do Instituto Oswaldo Cruz 84: 353-356. DOI: 10.1590/s0074-02761989000800063

- $\quad$ Merritt RW, Cummins KW (1996) An Introduction to the Aquatic Insects of North America. Kendall/Hunt Publishing, Dubuque, $1158 \mathrm{pp}$.

- Mittermeier R, Turner W, Larsen F, Brooks T, Gascon C (2011) Global Biodiversity Conservation: The Critical Role of Hotspots. Biodiversity Hotspots. Springer, Berlin. DOI: $10.1007 / 978-3-642-20992-51$

- Molineri C, Salles FF, Emmerich D (2015) Revision of Campsurus violaceus species group (Ephemeroptera: Polymitarcyidae) with new synonymies and nomina dubia in Campsurus Eaton, 1868. Zootaxa 3920 (1): 51. DOI: 10.11646/zootaxa.3920.1.3

- Monné ML, Ferreira VS, Thomas MC, Costa C, Ferreira-Jr N, Aloquio S, Lopes-Andrade C, Sandoval-Gómez VE, Pollock DA, Sekerka L, Linzmeier AM, Bulirsch P, Flores GE, McHugh J, Moura LA, Gimmel ML, Segura MO, Lord N, Constantin R, Almeida LM, Grossi PC, Grzymala TL, Biffi G, Vaz-de-Mello FZ, Caron E, Spiessberger EL, Bicho CL, Chandler DS, Mermudes JRM, Vanin SA, Bená DC, Cline A, Monné MA, Souza DS, Hájek J, Anichtchenko A, Ribeiro-Costa CS, Agrain F, Chamorro ML, Cupello M, Smith TR, Botero JP, Sampaio BHL, Passos MI, Vaz S, Colpani D, Benetti CJ, Leivas FWL, Degallier N, Gnaspini P, Nascimento EA, Quintino HYS, McElrath TC, Powell G, Escalona H, Barbosa FF, Casari S, Silva AAS, Shockley F, Manfio D, Slipinski A, Leschen RAB, Cruz LS, Nascimento FEL, Tomaszewska W, Newton AF, Peck SB, Silveira LFL, Hamada N, Johnson C, Castro-Guedes CF, Santos PB, Aragão AC, Puker A, Morse GE, Corrêa RC, Rosa SP, Ivie MA (2016) Coleoptera in Catálogo Taxonômico da Fauna do Brasil. http://fauna.jbri.gov.br/fauna/faunadobrasil/223. Accession date: 2016126.

- Moreira FFF (2016) Water Bugs Distributional Database. http://sites.google.com/site/ distributionaldatabase 
- Moreira FFF, Campos GGF (2012) New distributional data concerning some Gerromorpha (Insecta: Hemiptera: Heteroptera) from Brazil. Check List 8 (3): 542-547. DOI: $10.15560 / 8.3 .542$

- Moreira FFF, Barbosa JF, Ribeiro JRI, Alecrim VP (2011) Checklist and distribution of semiaquatic and aquatic Heteroptera (Gerromorpha and Nepomorpha) occurring in Brazil. Zootaxa 2958: 1-74. URL: URL: http://www.mapress.com/zootaxa/ list/2011/2958.html

- Mouchamps R (1959) Remarques concernant les genres Hydriobiomorpha Blackburn et Neohydrophilus Orchymont (Coléoptères Hydrophilides). Bulletin et annales de la Société royale d'Entomologie de Belgique 95: 295-335.

- Myers N, Mittermeier RA, Mittermeier CG, de Fonseca GAB, Kent J (2000) Biodiversity hotspots for conservation priorities. Nature 403: 853-858. DOI: $10.1038 / 35002501$

- Navás L (1916) Neuroptera nova americana. II Series. Memorie dell' Accademia pontificia dei Nuovi Lincei, Ser. 2 2: 71-80.

- Navás L (1924) Odonatos nuevos o interesantes. Memorias de la Real Academia de Ciencias y Artes de Barcelona 18: 315-332.

- $\quad$ Nieser N (1977) A revision of the genus Tenagobia Bergroth (Heteroptera: Corixidae). Studies on Neotropical Fauna and Environment 12 (1): 1-56. DOI: 10.1080/0165052770 $\underline{9360510}$

- $\quad$ Nobre CE, Carvalho AL (2014) Odonata of Itatira, a Brazilian semi-arid area in the state of Ceará. International Journal of Odonatology 17: 73-80. DOI: $\underline{10.1080 / 13887890.201}$ 4.907545

- $\quad$ Nobre CEB (2016) Erythrodiplax leticia: Description of the female and updated geographic distribution (Odonata: Libellulidae). Zootaxa 4067 (4): 469-472. [In English]. DOI: $10.11646 /$ zootaxa.4067.4.5

- $\quad$ Orchymont Ad (1943) Faune du nord-est brésilien (récoltes du Dr O. Schubart): Palpicornia. Musée royal d'histoire naturelle de Belgique 28 (2): 1-85.

- Paulson D (2003) Comments on the Erythrodiplax connata (Burmeister, 1839) group, with the elevation of $E$. fusca (Rambur, 1842), E. minuscula (Rambur, 1842), and $E$. basifusca (Calvert, 1895) to full species (Anisoptera: Libellulidae). Bulletin of American Odonatology 6 (4): 101-110.

- $\quad$ Perty JAM (1834) Delectus animalium articulatorum, quae in itinere per Brasiliam, annis MDCCXVII-MDCCCXX jussu et auspiciis Maximiliani Josephi I. DEMonachii: Impensis Editoris, Munich, 224 pp. DOI: http://dx.doi.org/10.5962/bhl.title.102991

- $\quad$ Pinto ÂP (2010) A Sertanejo's Trip: Occurrence of Orthemis sulphurata Hagen in Northeastern Brazil? Argia 22 (2): 12-14.

- Pinto ÂP (2016) Odonata in Catálogo Taxonômico da Fauna do Brasil. http:// fauna.jbri.gov.br/fauna/faunadobrasil/171. Accession date: 2016126.

- $\quad$ Pinto ÂP, Lamas CJE (2010) Navicordulia aemulatrix sp. nov. (Odonata, Corduliidae) from northeastern Santa Catarina State, Brazil. Revista Brasileira de Entomologia 54 (4): 608-617. DOI: 10.1590/s0085-56262010000400012

- $\quad$ Pinto ÂP, Lamas CJE (2011) Oligoclada mortis sp. nov. from Rondônia State, Brazil, and distributional records of other species of the genus (Odonata: Libellulidae). International Journal of Odonatology 14 (4): 291-303. [In English]. DOI: 10.1080/138878 90.2011 .629942

- $\quad$ Prado DE (2003) As Caatingas da América do Sul. In: Leal IR, Tabarelli M, da Silva JMC (Eds) Ecologia e Conservação da Caatinga. Recife, 822 pp. 
- $\quad$ Quinteiro FB, Costa AM, Calor AR (2014) Trichoptera do Semiárido II: Integripalpia. p. 229-244. In: Bravo F, Calor A (Orgs). Artrópodes do Semiárido: biodiversidade e conservação. Printmídia, Feira de Santana, 289 pp.

- Rafael JA, Câmara JT (2016) Empididae in Catálogo Taxonômico da Fauna do Brasil. $\underline{\text { h }}$ ttp://fauna.jbrj.gov.br/fauna/faunadobrasil/2379. Accession date: 2016126.

- Rafael JAR, Gorayeb IS (1982) Tabanidae (Diptera) da Amazônia, I - Uma nova armadilha suspensa e primeiros registros de mutucas de copas de árvores. Acta Amazonica 12 (1): 232-236.

- Ribeiro JRI (2000) Description of the male of Belostoma foveolatum and new records of B. costalimai and B. stollii (Heteroptera: Belostomatidae). Entomological News 3: 159-170.

- $\quad$ Ribeiro JRI (2007) A review of the species of Belostoma Latreille, 1807 (Hemiptera: Heteroptera: Belostomatidae) from the four southeastern Brazilian states. Zootaxa 1477: 1-70. DOI: http://dx.doi.org/10.11646/\%25x

- $\quad$ Righi-Cavallaro K, Froehlich C, Lecci L (2013) New species of Anacroneuria (Plecoptera: Perlidae) from northeast Brazil. Studies on Neotropical Fauna and Environment 48 (2): 125-134. DOI: 10.1080/01650521.2013.844590

- Rodrigues HDD, de Melo AL, Ferreira-Kepler RL (2012) New records of Gerromorpha (Insecta: Hemiptera: Heteroptera) from Brazil. Check List 8: 908-913. DOI: 10.15560/8. $\underline{5.908}$

- $\quad$ Rosenberg DM, Resh VH (1993) Freshwater Biomonitoring and Benthic Macroinvertebrates. Chapman \& Hall, London, $488 \mathrm{pp}$.

- Salles FF, Boldrini R (2016) Ephemeroptera in Catálogo Taxonômico da Fauna do Brasil. http://fauna.jbrj.gov.br/fauna/faunadobrasil/122. Accession date: 2016126.

- Salles FF, Massariol FC, Nascimento JMC, Boldrini R, Raimundi EA, Angeli KB, Souto PM (2016) Ephemeroptera do Brasil. http://ephemeroptera.com.br/

- Santos AM, Cavalcanti DR, da Silva JMC, Tabarelli M (2007) Biogeographical relationships among tropical forests in north-eastern Brazil. Journal of Biogeography 34 (3): 437-446. DOI: 10.1111/j.1365-2699.2006.01604.x

- Santos APM, Takiya D, Nessimian J (2016a) Integrative taxonomy of Metrichia Ross (Trichoptera: Hydroptilidae: Ochrotrichiinae) microcaddisflies from Brazil: descriptions of twenty new species. PeerJ 4: e2009. DOI: 10.7717/peerj.2009

- Santos APM, Calor AR, Dumas LL, Pes AMO, Souza WRM, Henriques-Oliveira AL, Camargos LM (2016b) Trichoptera in Catálogo Taxonômico da Fauna do Brasil. http:// fauna.jbri.gov.br/fauna/faunadobrasil/278

- Santos ND (1968) Descrição de Leptagrion dardanoi sp. n. (Odonata, Coenagrionidade). Atas da Sociedade de Biologia do Rio de Janeiro 12 (2): 63-65.

- Silva MS, Ferreira RL (2009) Caracterização ecológica de algumas cavernas do Parque Nacional de Ubajara (Ceará) com considerações sobre o turismo nestas cavidades. Revista de Biologia e Ciências da Terra 9 (1): 59-71.

- Souza WRM, Santos APM, Takiya DM (2014a) First records of Ochrotrichia Mosely, 1934 (Trichoptera: Hydroptilidae) in Northeastern Brazil: Five new species and two new geographical records. Zootaxa 3852 (2): 273-282. DOI: 10.11646/zootaxa.3852.2.6

- Souza WRM, Santos APM, Takiya DM (2014b) Three new species of Hydroptila (Trichoptera: Hydroptilidae) from Northeastern Brazil. Zoologia (Curitiba) 31 (6): 639-643. DOI: 10.1590/s1984-46702014000600010 
- Souza WRM, Santos APM, Takiya DM (2016a) Description of a new species of Betrichia Mosely 1939 from Brazil and edescription of the type-species (Trichoptera: Hydroptilidae: Leucotrichiinae). Zootaxa 4061 (3): 391-395. DOI: 10.11646/ zootaxa.4061.3.9

- Souza WRM, Santos APM, Takiya DM (2016b) Three new species of Stactobiinae (Trichoptera: Hydroptilidae) with the first record of the genus Orinocotrichia Harris, Flint \& Holzenthal from Brazil. Zootaxa in press: 0.

- Tabarelli M, Vicente A (2004) Conhecimento sobre plantas lenhosas da Caatinga: lacunas geográficas e ecológicas. In: da Silva JMC, Tabarelli M, Fonseca M, Lins L (Eds) Biodiversidade da Caatinga: áreas e ações prioritárias para conservação. Ministério do Meio Ambiente, Brasília.

- Truxal FS (1949) A study of the genus Martarega (Hemiptera: Notonectidae). Journal of the Kansas Entomological Society 22 (1): 1-36. URL: URL: http://www.jstor.org/ stable/25081875

- Truxal FS (1953) A revision of the genus Buenoa (Hemiptera: Notonectidae). University of Kansas Science Bulletin 35: 1351-1517.

- $\quad$ Vasconcellos A, Andreazze R, Almeida A, Araujo HP, Oliveira E, Oliveira U (2010) Seasonality of insects in the semi-arid Caatinga of northeastern Brazil. Revista Brasileira de Entomologia 54 (3): 471-476. DOI: 10.1590/s0085-56262010000300019

- Vianna DM, De Marco Júnior P (2012) Higher-Taxon and Cross-Taxon Surrogates for Odonate Biodiversity in Brazil. Natureza \& Conservação 10 (1): 34-39. DOI: $10.4322 /$ natcon.2012.006

- $\quad$ von Ellenrieder N (2013) A revision of Metaleptobasis Calvert (Odonata: Coenagrionidae) with seven synonymies and the description of eighteen new species from South America. Zootaxa 3738 (1): 1. [In English]. DOI: 10.11646/zootaxa.3738.1.1

- Wang X, Auler A, Edwards RL, Cheng H, Cristalli P, Smart P, Richards D, Shen C (2004) Wet periods in northeastern Brazil over the past 210 kyr linked to distant climate anomalies. Nature 432 (7018): 740-743. DOI: 10.1038/nature03067

- Werneck F, Nogueira C, Colli G, Sites J, Costa G (2012) Climatic stability in the Brazilian Cerrado: implications for biogeographical connections of South American savannas, species richness and conservation in a biodiversity hotspot. Journal of Biogeography 39 (9): 1695-1706. DOI: 10.1111/j.1365-2699.2012.02715.x

- Westfall MJ (1992) Notes on Micrathyria, with descriptions of M. pseudeximia sp. n., M. occipita sp. n., M. dunklei sp. n. and M. divergens sp. n. (Anisoptera: Libellulidae). Odonatologica 21: 203-218. 